Abstracts of the

0

6th International Conference on Behavioral Addictions

1

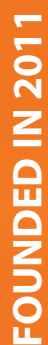

Journal of

Behaviora

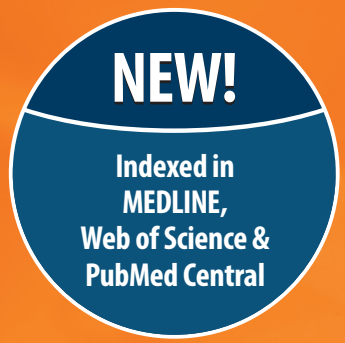

Addictions

Editor-in-Chief:

Zsolt Demetrovics

Eötvös Loránd University

Institute of Psychology

9 


\title{
Journal of Behavioral Addictions
}

\author{
Editor-in-Chief \\ ZSOLT DEMETROVICS (Eötvös Loránd University, Hungary)
}

\begin{abstract}
Associate Editors
Judit Balázs (Eötvös Loránd University, Hungary); Joël Billieux (University of Luxembourg, Luxembourg); Matthias Brand (University of Duisburg-Essen, Germany); Anneke Goudriaan (University of Amsterdam, The Netherlands); Daniel King (University of Adelaide, Australia); Ludwig Kraus (Institute for Therapy Research, Germany); Astrid Müller (Hannover Medical School, Germany); Halley M. Pontes (University of Tasmania, Australia); Marc N. Potenza (Yale University, USA); Attila Szabó (Eötvös Loránd University, Hungary); Róbert Urbán (Eötvös Loránd University, Hungary); Aviv M. Weinstein (Ariel University, Israel)
\end{abstract}

\section{Editorial Board}

Max W. Abbott (Auckland University of Technology, New Zealand); Elias N. Aboujaoude (Stanford University School of Medicine, USA); Hojjat Adeli (Ohio State University, USA); Alex Baldacchino (University of Dundee, United Kingdom); Alex Blaszczynski (University of Sidney, Australia); Kenneth Blum

(University of Florida, USA); Henrietta Bowden-Jones (Imperial College, United Kingdom); Gerhard Bühringer (Technische Universität Dresden, Germany); Wim van den Brink (University of Amsterdam, The Netherlands); Sam-Wook Choi (Eulji University, Republic of Korea); Damiaan Denys (University of Amsterdam, The Netherlands); Jeffrey L. Derevensky (McGill University, Canada); Naomi Fineberg (University of Hertfordshire, United Kingdom); Marie Grall-Bronnec

(University Hospital of Nantes, France); Jon E. Grant (University of Minnesota, USA); Mark Griffiths (Nottingham Trent University, United Kingdom); Heather Hausenblas (Jacksonville University, USA); Tobias Hayer (University of Bremen, Germany); Susumu Higuchi (National Hospital Organization Kurihama Medical and Addiction Center, Japan); David Hodgins (University of Calgary, Canada); Eric Hollander (Albert Einstein College of Medicine, USA); Jaeseung Jeong (Korea Advanced Institute of Science and Technology,

Republic of Korea); Yasser Khazaal (Geneva University Hospital, Switzerland); Orsolya Király

(Eötvös Loránd University, Hungary); Emmanuel Kuntsche (Addiction Info Switzerland,

Switzerland); Michel Lejoyeux (Paris University, France); Frederick Gerard Moeller (University of Texas, USA); Daniel Thor Olason (University of Iceland, Iceland); Nancy Petry (University of Connecticut, USA); Bettina Pikó (University of Szeged, Hungary); Afarin Rahimi-Movaghar (Teheran University of Medical Sciences, Iran);

József Rácz (Hungarian Academy of Sciences, Hungary); Rory C. Reid (University of California Los Angeles, USA); Hans-Jürgen Rumf (University of Lübeck, Germany); Marcantanio M. Spada (London South Bank University, United Kingdom); Daniel Spritzer (Study Group on Technological Addictions, Brazil); Dan J. Stein (University of Cape Town, South Africa); Sherry H. Stewart (Dalhousie University, Canada); Attila Szabó (Eötvös Loránd University, Hungary); Ferenc Túry (Semmelweis University,

Hungary); Alfred Uhl (Austrian Federal Health Institute, Austria); Johan Vanderlinden

(University Psychiatric Center K.U. Leuven, Belgium); Alexander E. Voiskounsky

(Moscow State University, Russia); Kimberly Young (Center for Internet Addiction, USA)

\section{Editorial Assistant \\ Csilla Ágoston (Eötvös Lóránd University, Hungary)}

6th International Conference on Behavioral Addictions Volume 8, Number 1, June 2019

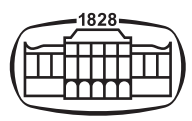

AKADÉMIAI KIADÓ

MEMBER OF WOLTERS KLUWER GROUP 


\section{Contents}

\section{6th International Conference on Behavioral Addictions June 17-19, 2019 \\ Yokohama, Japan}

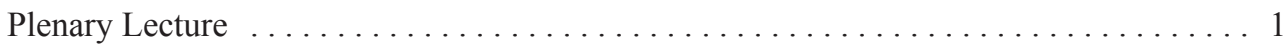

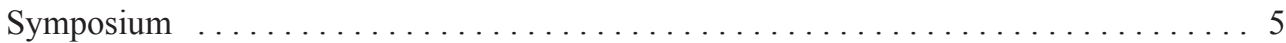

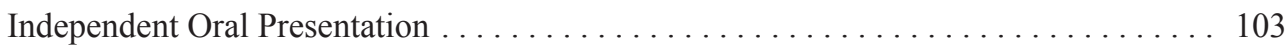

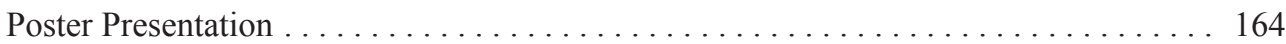

Training Seminar on Behavioral Addictions $\ldots \ldots \ldots \ldots \ldots \ldots \ldots \ldots \ldots \ldots \ldots \ldots \ldots \ldots$

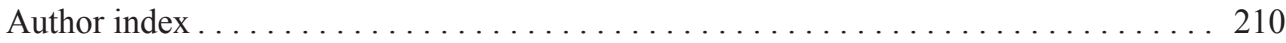




\title{
PLENARY LECTURE
}

\section{PL1}

\section{Public Health Implications of Addictive Behaviours and Substance Use}

\author{
Vladimir B. Poznyak* \\ Department of Mental Health and Substance Abuse, World Health Organization (WHO) \\ poznyakv@who.int
}

There is a conceptual integration of disorders due to substance use and addictive behaviours in the 11th revision of the International Classification of Diseases (ICD-11), which is based on similarities in their neurobiology, phenomenology, natural history, comorbidity profiles and public health implications. Conceptual framework for public health impact of alcohol use can be applied for assessment of the public health implications of addictive behaviors considering available epidemiological data. The clinical and public health utility of a spectrum of substance use behaviours is well established, including hazardous and harmful substance use and dependence. There is emerging evidence that a similar approach is useful for addictive behaviours. With the inclusion of gaming and gambling disorders as well as hazardous gaming and gambling in ICD-11, the available evidence does not yet provide sufficient justification for defining harmful gaming or gambling as potential diagnostic categories for ICD-11 despite similarities in conceptual approaches to addictive behaviours and substance use. Studies that address clinical and public health utility of gaming and gambling behaviours resulting in negative health consequences that do not meet the ICD-11 criteria for gaming or gambling disorder are urgently needed to inform future incremental revisions of international classification of mental and behavioural disorders. Further development of easy to use diagnostic algorithms based on well-substantiated diagnostic boundaries between health conditions associated with addictive behaviours is another priority for international research. Following the inclusion of gaming disorder in ICD-11, work has commenced, under the auspices of $\mathrm{WHO}$, on the development of a robust and empirically-based screening instrument and diagnostic interview for gaming disorder that can be used in different cultures and health care systems. This will facilitate international comparisons. Public health measures aimed at reducing the negative impact of addictive behaviours on population health are also under development in line with policy measures implemented for alcohol and drug use, but their effectiveness and cost-effectiveness still need to be demonstrated in diverse settings. The role of different stakeholders in the development of normative guidance and the implementation of policy measures to reduce the negative health consequences of addictive behaviours should be considered while taking into account commercial determinants of health. The lessons learned from similar processes for the harmful use of alcohol and tobacco need to be considered despite differences between substance use and addictive behaviours. There is an increasing global awareness of the public health implications of addictive behaviours, but a strong evidence base is needed for the development, acceptance and implementation of public health measures to reduce the health burden of addictive behaviours which stakeholders may perceive differently. 


\title{
PL2
}

\section{The diagnosis of DSM-5 Internet gaming disorder or ICD-11 gaming disorder.}

\author{
Chih-Hung Ko*
}

Department of Psychiatry, Kaohsiung Municipal Hsiao-Kang Hospital, Kaohsiung Medical University cyberko@seed.net.tw

The Diagnostic and Statistical Manual of Mental Disorders, Fifth Edition (DSM-5) proposed the diagnostic criteria for Internet gaming disorder (IGD) to define Internet gaming addiction. Based on these criteria, the global prevalence ranges between $0.5 \%$ and $6 \%$ [1]. The concerns regarding the threshold, validity, and utility of the DSM-5 IGD criteria had been raised in the past years. The International Classification of Diseases 11th Revision (ICD-11) included its own definition of gaming disorder (GD) at 2018. Impaired control, increased priority, and negative consequences should be presented with marked functional impairment for more than one year. The ICD criteria was based on clinical evidence and interests of public health. More scientific evidence and information were required to support and improve its validity and utility. The presenting speech would focus on diagnosis of internet gaming disorder and its clinical utility.

\section{PL3}

\section{Behavioural addictions: need for a conceptual framework}

\section{Alexander Blaszczynski*}

The University of Sydney

alex.blaszczynski@sydney.edu.au

The concept of behavioural addictions, mooted in the earlier 1990's, has witnessed an exponential growth in academic attention culminating in the successful inclusion of at least one behaviour, gaming disorder in ICD-11 and the inclusion of a new category of non-substance related addictions in DSM-5. Although there is no debate about the extent to which some behaviours carried out to excess have the capacity to generate a range of harms, controversy exists as to whether behavioural addictions constitute a disease, an illness or social construct. As a result, there are a myriad of terminology used to describe the same 'disorder', criteria used for one behaviour adapted for use to diagnose another, investigation of psychosocial, personality and neurobiological variable as etiological explanations, and limited empirical evidence supporting treatment interventions. The risk, as some researchers have highlighted, is in overpathologizing normal behaviours, and trivialising the concept of behavioural addictions. In this presentation, it is argued that attention should be directed towards a cohesive and integrated set of guidelines applied in defining, measuring and treating specific behavioural addictions, and clarifying respective role of product providers, governments, families, communities and welfare providers. Given the complexities of some behaviours, it is necessary to develop a multi-disciplinary framework setting out the relative responsibilities of key 
stakeholders (e.g., researchers, community groups, governments, policy makers, treatment providers, the industry, families and individuals) that can be used to inform and implement policies for those behaviours that represent important social and public health issues, for example, gaming and gambling.

\title{
PL4
}

\section{Public Health Approach for Internet Use Disorders}

\author{
Hae Kook Lee ${ }^{1 *}$, SulKi Chung' ${ }^{2}$ Sun-Jin Jo \\ ${ }^{1}$ Department of Psychiatry, Uijeongbu St.Mary's Hospital, The Catholic University of Korea, Seoul, \\ Korea \\ ${ }^{2}$ Chung-Ang University, Department of Social Welfare \\ ${ }^{3}$ Department of Preventive Medicine, The Catholic University, Korea \\ nplhk@catholic.ac.kr
}

With the increasing use of the Internet throughout our daily lives, the Internet use disorders (IUD) are gaining recognition. With the growing population of Internet and smartphone users, the public health concerns over IUD also increased in regard to adverse cognitive, developmental, psychosocial, and physical consequences. The author provides research evidence shown in the theoretical conceptual model of IUD based on public health model and continuum model of addictive disorders. Addictive online-media components and predisposing biologic, cognitive, and psychological risks put individuals at higher risk of developing IUD. In addition, environmental factors may also contribute to IUD. Therefore, prevention and intervention effort should not only target the 'host' (individual vulnerabilities) but also the 'agent' (media related-risk) and the environmental risk to better address the complex phenomena of IUD. Intervention approaches to IGD need to be provided according to seriousness of problems from social use to disordered use, respectively like primary intervention and tertiary intervention. Furthermore, based on the conceptual model of IGD, the multidisciplinary experts from different areas like prevention, treatment, education and consumer protection, etc. can contribute to effective strategy to reduce the harmful use of internet, and promote healthy use of digital media and device.

\section{PL5}

\section{Where to draw the line: Development of Low risk gambling guidelines}

\author{
David C. Hodgins* \\ University of Calgary \\ dhodgins@ucalgary.ca
}

People who gamble are advised to set personal gambling limits to avoid gambling-related harm to themselves and others. Increasingly, tools are available to help gamblers to set limits. However, compared to other public health areas (drinking alcohol, diet guidelines), this 
limit-setting advice is general and non-quantitative. A common responsible gambling slogan is: "set a limit and stick within it". In this presentation, I will be review research that has attempted to quantify the association between increasing gambling involvement - frequency, time spent gambling, expenditure - and the experience of gambling- related harms. The risk curve methodology for identifying cut-off for riskier gambling will be described, including strengths and limitations. Current work to develop Canadian limits, collaborating with international partners, will be presented.

\title{
PL6
}

\section{Neuroimaging of Gambling Disorder: Toward precision addiction psychiatry}

\author{
Hidehiko Takahashi* \\ Tokyo Medical and Dental University \\ hidepsyc@tmd.ac.jp
}

Gambling disorder (Gambling addiction) has been categorized as a behavioral addiction in the international diagnostic criteria of mental disorders. This shift was partly due to the fact that gamble addiction and substance addiction have much in common in clinical symptoms / brain science findings. In this lecture, we will show the common as well as differential findings between gamble addiction and substance addiction revealed by brain imaging research. There might be various subtypes of gambling addiction. In the second half, we will present an example of identification of gambling addiction subtype suggested by our decision-making and brain imaging research. We also want to discuss the possibility of an fMRI imaging biomarker of gambling disorder. 


\title{
SYMPOSIUM
}

SY1-1

\section{Opening of casinos in Japan: possible impact on issues related to gambling}

\author{
Kotaro Nishimura*, Takanobu Matsuzaki, Sachio Matsushita, Susumu Higuchi \\ National Hospital Organization, Kurihama Medical and ddiction Center \\ kotan2001@gmail.com
}

In Japan, only official gambling acts had been allowed for a long time. But, on July 20th 2018, the Japanese Diet enacted a law allowing private companies to operate casinos. Casinos will most likely be opened by the year 2024 in Japan, which may have a great impact on issues related to gambling. This presentation will review existing literatures on the relationship between casino opening and the occurrence of gambling related problems and discuss possible consequences of casino opening in Japan.

\section{SY1-2}

\section{Understanding Subsyndromal Gambling Disorder}

\section{Jon Grant*}

University of Chicago

jongrant@uchicago.edu

Background: Understanding the chain of progression from recreational gambling to gambling disorder is vital towards understanding the underlying biological mechanisms (pathogenesis). Comparing people with gambling disorder with those at an increased risk of developing gambling disorder would help to elucidate whether neurobiological aspects of gambling disorder are evident prior to the development of overt pathology or stem from the disorder itself, perhaps even reflecting the harmful effects of recurrent gambling on brain function.

Methods: In this talk we address current research related to neurocognition and neuroimaging in adults with gambling disorder as well as those with subsyndromal forms of gambling disorder.

Results: Recent research demonstrates morphologic abnormalities of pallidum and putamen in those with gambling disorder symptoms even without meeting full diagnostic criteria of gambling disorder. Other research suggests that selective cognitive dysfunction may already be present in terms of decision-making in at-risk gamblers, even before psychopathology arises. Discussion: Symptoms of gambling disorder may necessitate interventions as there are data linking subsyndromal gambling and gambling disorder. 
SY1-3

\title{
Network Models of Gambling Symptoms: New Statistical Applications and Clinical Implications
}

\author{
Joshua B Grubbs 1*, Heather Chapman $^{2}$ \\ ${ }^{1}$ Department of Psychology, Bowling Green State University, Bowling Green, OH, USA \\ ${ }^{2}$ Gambling Treatment Program, Louis Stokes Cleveland Department of Veteran Affairs Medical \\ Center, Cleveland, $\mathrm{OH}$, USA \\ GrubbsJ@BGSU.edu
}

Recent advances in statistical modeling and understandings of psychological symptoms have begun to alter longstanding conceptualizations of well-known disorders, such as depression (Fried et al., 2016). To date, such models have not been applied to behavioral addictions, such as Gambling Disorder. Using two samples, an inpatient sample of veterans receiving treatment for gambling disorder and an online convenience sample of adult recreational gamblers, network models are applied to gambling symptoms. Although the centrality of symptoms varied quite substantively between recreational gamblers and clinical gamblers, symptoms such as chasing losses, perceived loss of control, and lost time at work or school due to gambling behaviors were central in both groups.

\section{SY1-4}

\section{Clinical characteristics of veterans with gambling disorders seeking pain treatment}

\author{
Shane Winfield Kraus ${ }^{1 *}$, Silvia Ronzitti ${ }^{2}$, Suzanne Decker², Lisham Ashrafioun ${ }^{3}$ \\ ${ }^{1}$ VISN 1 New England MIRECC, Edith Nourse Rogers Memorial Veterans Hospital, \\ 200 Spring Road, Building 5, Room 135B, Bedford MA, 01730, United States \\ ${ }^{2}$ Yale School of Medicine, VA Connecticut Healthcare System, West Haven, Connecticut \\ ${ }^{3}$ VA VISN 2 Center of Excellence for Suicide Prevention, Canandaigua VA Medical Center \\ swkraus@gmail.com
}

Background and aims: We investigated the associations between gambling disorder, pain, and suicide attempts among US military veterans using Veterans Health Administration) pain-related services.

Methods: Retrospective cohort analysis of 221,817 veterans using pain services were included in the study analysis. We first analyzed differences in sociodemographic and clinical characteristics (i.e., psychiatric comorbidities and pain-related variables) according to gambling disorder. Next, we conducted logistic regression analyses to test the association between gambling disorder and suicide attempts.

Results: Female sex, depressive, alcohol, drug and tobacco use disorders are positively associated with a gambling diagnosis, whereas severe pain score was negatively associated with gambling disorder. Logistic regression analysis indicated that gambling disorder diag- 
nosis was associated suicide attempt in veterans who received a visit for pain in a VA hospital in the past year.

Conclusions: Our findings suggest that gambling disorder in female veterans and suicide attempts in veterans with gambling disorder warrants further consideration.

\title{
SY1-5
}

\section{Blurred boundaries between gam(bl)ing and other potentially addictive behaviors}

\author{
Marc N. Potenza* \\ Yale University \\ marc.potenza@yale.edu
}

Video or internet-based gaming is a prevalent behavior, particularly among adolescents and young adults. Aspects of gaming (e.g., participation in social casino games, microtransaction in games) have been linked to the initiation of gambling. Additionally, gambling-related elements that have been introduced into games (e.g., loot boxes or loot crates) have been debated in clinical and legal settings regarding the extent to which they warrant close regulation (or made illegal) given their potential impact upon health and development. Further, competitive gaming (eSports) has become very popular among adolescents and young adults, with career opportunities in competitive gaming a reality. In this setting, a small yet sizable proportion of individuals develop problems with gaming and gambling. This presentation will review the intersection of gaming and gambling, consideration relationships with other behavioral addictions and discuss public health and clinical implications.

\section{SY2-1}

\section{Technology-Enabled Personality Traits and Post-Privacy Psychology}

Elias Aboujaoude ${ }^{1 *}$, Yasser Khazzal ${ }^{2}$, Vladan Starcevic ${ }^{3}$, Philip Tam ${ }^{4}$

${ }^{1}$ Stanford University School of Medicine

${ }^{2}$ University of Geneva

${ }^{3}$ University of Sydney

${ }^{4}$ The Delta Clinic, Sydney

eaboujaoude@stanford.edu

Background and aim: Aggression, impulsivity and narcissism are personality traits nurtured by Internet technologies, and are expressed over platforms that often lack privacy protections. What may be the psychological consequences?

Methods: Literature review and conceptual synthesis.

Results: Cyberbullying, video game violence and online suicide have been conceptualized as manifestations of a more aggressive online personality. Also, the prevalence of im- 
pulse control disorders such as compulsive buying disorder and gambling disorder may have increased as a result of Internet-exacerbated impulsivity. Further, social media have been blamed for a rise in narcissism. The absence of privacy online can give such problematic behaviors a permanence that complicates therapeutic interventions. In addition, the psychological functions that are thought to require privacy may be compromised by the post-privacy online world, potentially also destabilizing psychological balance. Yet these important aspects of online psychology have gone largely ignored by "Internet addiction" research.

Conclusions: The online nurturing of negative personality traits and privacy compromises may have contributed to an urge- driven, less empathic, more narcissistic, and overly public culture. The far-reaching consequences make research into broader aspects of online psychology a priority.

\title{
SY2-3
}

\section{Cyberchondria: An overlooked type of problematic Internet use?}

\author{
Vladan Starcevic ${ }^{*}$, Stephanie Baggio ${ }^{2}$, Yasser Khazaal ${ }^{2}$, David Berle \\ Kirupamani Viswasam ${ }^{4}$ \\ ${ }^{1}$ University of Sydney \\ ${ }^{2}$ University of Geneva \\ ${ }^{3}$ University of Technology Sydney \\ ${ }^{4}$ Nepean Hospital \\ vladan.starcevic@sydney.edu.au
}

Objectives: This presentation will examine theoretical and empirical underpinnings of the link between cyberchondria and problematic Internet use (PIU).

Findings: Cyberchondria refers to repeated and / or excessive online health searches that are associated with increasing levels of health anxiety. There are certain similarities between cyberchondria and PIU. Both are time-consuming and characterised by preoccupation with and difficulty disengaging from an online activity. The negative consequences of cyberchondria are akin to those associated with PIU and include neglect of commitments, conflicts with others and impaired functioning. In cyberchondria, the seeking and receiving of healthcare can be affected, with disrupted communication and relationships with healthcare providers. Given that PIU encompasses a variety of online activities, cyberchondria may be a specific form of PIU. The relationship between cyberchondria and PIU has hardly been studied. However, a strong correlation between cyberchondria and PIU has been reported, and individuals with cyberchondria exhibited more prominent PIU compared to those who made online health searches without developing cyberchondria. A more recent research has confirmed the link between cyberchondria and PIU using different methodological approaches. It remains to be ascertained whether and to what extent levels of health anxiety may mediate this relationship. 


\title{
SY2-4
}

\section{Problematic Internet Use and Gaming Disorder: Mental Illnesses for the 21st Century}

Philip G Tam*

The Delta Clinic, Sydney

philip_g_tam@hotmail.com

Background and aims: To explore and summarise the initial development and subsequent evolution of the clinical concept of problematic internet use, or Gaming Disorder, and to locate it in its broader psychiatric, social and educational context. Method: Literature review; conceptual and clinical synthesis.

Results: The first clinical case-reports of what was initially dubbed 'internet addiction' emerged in North America in the mid-1990's, around the time that the 'World Wide Web' was developing. A growing clinical and public-health problem was observed in many other regions and countries, alongside an increasing research interest and body of literature. A major development arose with the conceptualisation of 'Internet Gaming Disorder' in the DSM 5, and 'Gaming Disorder' in the ICD 11 classification systems - steps that were not without controversy both from within the research community and the general public. The constant evolution of technology, and the increasing pervasiveness of these technologies in people's daily lives, shows that initial methods of conceptualising, researching and treating these disorders were often simplistic, and the emergence of novel clinical phenomena such as cyberchondria and nomophobia and the 'online disinhibition effect' require us to envision these disorders in a much broader, more complex ecosystem.

\section{SY3-1}

\section{The Effectiveness of the SBIRT Intervention on the High-Risk Group of Students for the Internet-Smartphone Addiction in the Community}

\author{
Soo-Young Bhang* \\ Eulji University, Eulji Hospital \\ bsy1@eulji.ac.kr
}

Objectives: Screening, Brief Intervention, and Referral to Treatment (SBIRT) is a good example of community involvement model for substance and behavior addiction. The purpose of this study is to investigate the effectiveness of the SBIRT model for intervention in schools and local child care centers.

Methods: From March to October 2017, we surveyed 3,937 students attending schools in Nowon-gu, who were smartphone users. Among them, 180 students were regarded as a highrisk group for addiction and were enrolled in a short-term group intervention program with the permission of their parents.

Results: After a short-term intervention program, a significant decrease in the smartphone dependence scale from 16.72 to 14.93 was observed. Male students showed a significant 
decrease from 17.53 to 15.12 , but female students' scores revealed an insignificant decrease from 15.73 to 14.71 . The effect of intervention by the school showed a significant decrease from 16.22 to 14.31 in elementary school students. A significant decrease was also present in middle school students whose scores declined from 18.50 to 15.88 .

Conclusions: We have demonstrated the efficacy of the SBIRT model in the local community in order to introduce evidence-based intervention program.

\title{
SY3-2
}

\section{Effect of transcranial direct current stimulation (tDCS) on craving and impulsivity in Internet gaming disorder}

\author{
Dai-Jin Kim ${ }^{*}$, Jo-Eun Jeong ${ }^{2}$, Chang-hyun Park ${ }^{3}$, Minji Kim ${ }^{4}$, Hyun Cho ${ }^{4}$, Arom Pyeon ${ }^{4}$, \\ Suji Jung, Jin-Young Kim ${ }^{4}$, Jihye Choi ${ }^{4}$, Ji-Won Chun ${ }^{4}$ \\ ${ }^{1}$ Department of Psychiatry, Seoul St. Mary's Hospital, College of Medicine, \\ The Catholic University of Korea \\ ${ }^{2}$ Department of Psychiatry, Daejeon St. Mary’s Hospital, College of Medicine, The Catholic \\ University of Korea \\ ${ }^{3}$ Center for Neuroprosthetics and Brain Mind Institute, Swiss Federal Institute of Technology (EPFL) \\ ${ }^{4}$ Addiction Laboratory, Department of Psychiatry, College of Medicine, The Catholic University \\ of Korea \\ ${ }^{5}$ Department of Psychology, Chung-Ang University \\ goodi11@hanmail.net
}

This study aimed to investigate the effect of transcranial direct current stimulation (tDCS) on reducing craving and impulsivity in Internet gaming disorder (IGD). 24 males with IGD were enrolled. Each participant received either the real or sham tDCS for a total of 10 sessions. The anode of tDCS was placed over Rt. dorsolateral prefrontal cortex (DLPFC). All participants were assessed for a visual analog scale to measure the craving. To measure impulsivity, Barratt impulsiveness scale (BIS)-11 and stop signal task (SST) were conducted at the pre- and post-stimulation sessions. To evaluate the differences in brain connectivity of pre- and posttDCS between the real and sham group, resting-state fMRI scanning was conducted twice. With respect to craving, only a significant effect of time was observed. With respect to impulsivity, however, a significant effect of tDCS condition on BIS-11 and the numbers of stop trial errors committed was observed. The real group showed decreased connectivity between Rt. DLPFC and anterior cingulate cortex (ACC) relative to the sham group in post-stimulation versus pre-stimulation. In addition, the connectivity between the ACC and the Rt. inferior frontal gyrus which is widely thought to be involved in response inhibition was negatively correlated with the numbers of stop trial errors in SST. These findings suggest that stimulation of the Rt. DLPFC with tDCS can improve impulsivity in IGD, supporting the treatment option for tDCS in IGD. 
SY3-3

\title{
Effectiveness of a School-based Brief Intervention for the Prevention of Internet Addiction
}

\author{
Hideki Nakayama*, Takanobu Matsuzaki, Satoko Mihara, Takashi Kitayuguchi, \\ Susumu Higuchi \\ Kurihama Medical and Addiction Center \\ matimi19750519@hotmail.com
}

Aims: The purpose of this study was to evaluate the possible effect of a brief school-based group intervention to prevent Internet addiction (IA).

Methods: All the study participants were second-grade students $(\mathrm{N}=182)$ at a boys' private junior high school. The Internet addiction intervention, provided in June 2018, was divided into two sessions. In the first session, lecture on Internet addiction were delivered in a mass teaching style. In the second session, students discussed about appropriate use of Internet in a workshop style. Questionnaire surveys were conducted just before and 5 months after the intervention. A total of 114 students participated in all the procedures and were included in the data analyses. Results: A significant increase in the average Internet and game use time was observed between the two surveys. Furthermore, a significant reduction in the average Young's Internet Addiction test score and significant increase in the average degree of drowsiness during the daytime were also observed.

Conclusions: This brief intervention did not have any long-term effect across the summer vacation. We consider that a more longitudinal or earlier intervention may be more effective.

\section{SY3-4}

\section{Rebootcamp: there is no wifi in the forest, but we will guarantee you a better connection}

\author{
Huub Boonen ${ }^{1 *}$, Joel Billieux ${ }^{2}$ \\ ${ }^{1}$ UC-Leuven-Limburg, CAD Limburg \\ ${ }^{2}$ University of Luxembourg \\ Huub.boonen@ucll.be
}

An innovating type of early intervention targeting problematic video gaming that is developed and tested in Belgium, consists in "Rebootkamps". These camps target teenagers who are struggling with life because of their high involvement in gaming. "Rebootkamps": attractive programme for gamers, including adventurous activities, outdoor gaming, social skills training, self-discovery group sessions and various activities promoting group dynamics. Bringing together gamers and therapists / prevention workers in this five-day experience is 
aimed to help the gamers in modifying their self-perception, developing coping strategies to face negative emotions or stressful situations, and re-appraising gaming involvement according to academic and personal objectives and goals. Effects are reached through experiential learning rather than via formal therapy. Theoretically anchored in the self-determination theory, Rebootkamps focus on the fulfillment of the basic psychological needs of Autonomy, Belongingness and Competence as an alternative to gaming behavior. The inclusion of parental guidance and acknowledging the pivotal role of the context in gaming involvement are two elements that make Rebootkamp a highly innovative approach for dealing with problematic gaming issues. This early intervention can be seen as a mean to reduce treatment gap and promote help-seeking in adolescents and children struggling with their gaming behaviors.

\title{
SY4-1
}

\section{Group psychotherapy for Gambling Disorder in a local mental health and welfare center in Japan}

\author{
Hitoshi Tanabe*, Daisuke Okazaki \\ Hokkaido Prefectural Mental Health and Welfare Center \\ h-tanabe@hokusei.ac.jp
}

We started group psychotherapy (GPT) for Gambling Disorder (GD) in Hokkaido Prefectural Mental Health and Welfare Center in 1990s. The reasons why we started GPT by ourselves are that we had no other available treatments and no self- help groups in our area at that time, and that we wanted to ascertain in this talk therapy whether GD has the same quality as other addictive disorders or not.

We have group sessions at night, because almost all members have to work hard to pay their debt in the daytime. We applied individual psychotherapy to the group session members if necessary. The group session members had many problems such as bankruptcy, unemployment, divorce, stealing, embezzlement, and often they have suicidal thoughts and attempts.

We have recognized GD is a persistent and recurrent problematic gambling leading to clinically significant impairment or distress. We understand GD is an important public health issue in Japan. But most of the public health professionals in Japan don't realize it seriously. We would like to make use of our experience for the prevention against and early intervention to GD in community mental health. 


\title{
SY4-2
}

\section{Approach to gambling addiction in Nagano prefecture mental health welfare center}

\author{
Noriaki Koizumi ${ }^{*}$ \\ Mental Health and Welfare Center in Nagano Prefecture \\ koizumi-noriaki@pref.nagano.lg.jp
}

The Mental Health and Welfare Center in Nagano Prefecture has undergone a variety of projects with the increase of consultations on gambling disorder. In this presentation we will introduce organizational development of GA (Gamblers Anonymous), management of addiction group therapy and family group therapy, verification of effect of addiction group therapy, holding of family classes, dissemination of awareness in community mental health, and Liaison meeting of regional stakeholders.

\section{SY4-3}

\section{Initiatives of Mental Clinics Regarding Gambling Disorder}

\section{Taku Sato*}

Naruse Mental Clinic, psychiatrist

naruse-cl@royal.ocn.ne.jp

It is widely known that the use of Gamblers Anonymous and rehabilitation facilities is effective for gambling disorder, but the role of psychiatric medical institutions is unlimited and remains unclear. There are regional differences in the number of Gamblers Anonymous and rehabilitation facilities, and it can be surmised that the roles required by psychiatric medical institutions differ in regions where local resources are abundant and regions where there are few resources. The mental clinic with which I am affiliated is located in an area that has abundant resources such as Gamblers Anonymous, Gam-Anon, and rehabilitation facilities, and it is desirable to provide support services for gambling disorder different from these. Here, we report one method of responding to gambling disorder in psychiatric medical institutions other than the provision of group meetings. 
SY4-4

\section{Treatment of patients with gambling disorders (GDs) in Kitasato University East Hospital (KUEH)}

\section{Takafumi Asakura*}

Kitasato University East Hospital, Department of Psychiatry / Kitasato University Graduate School of Medical Sciences

kingmelanin@icloud.com

KUEH is not specialized for addiction, but has been treating gambling disorders (GDs) since 2014. In order to avoid confrontation with patients and to motivate them, we do not discuss whether they can be diagnosed as having a GD, but treat the gambling that is the cause of the problems as "behavior" and explain what is happening to them using behavioral economics. In addition, we propose alternate behaviors by focusing on the behavior contingencies. As a result, the gambling behavior in such patients has been greatly reduced. We started our own group therapy program in 2017 with the aim of alleviating patients' sense of refusal for Self-care-group by generalizing stimulus. In this group therapy program, we take advantage of the knowledge of interventions on addictive behaviors from prior experience and provide a place for patients to connect with others who are continuously participating in self-help groups. Furthermore, close cooperation with the mental health welfare center and Addiction rehabilitation centers such as DARC has been strengthened so that even after the final examination, patients still have access to support. At this symposium, I would like to introduce the KUEH trial.

\section{SY4-5}

\section{Dissemination and implementation of CBT program for Gambling Disorder in Mental Health and Welfare Centers in Japan}

\section{Keiji Kobara*, Hiroshi Sato}

Shimane Prefectural Counseling Center for Physical and Mental Health keijikobara@gmail.com

There are few medical institutions that can implement cognitive behavioral therapy for gambling disorder in Japan. To complement this, 69 local mental health and welfare centers (MHWCs) are supposed to give professional consultation service and recovery programs for gambling disorder (GD) patients. However, it was difficult to solve this problem because of lack of skills of MHWC staff and lack of executable programs. Therefore, we tried to solve the problem by developing a cognitive behavioral therapy program which can be carried out even by inexperienced staff, and by training MHWC staff nationwide. At the symposium, we will report on the details of the training content and the number of MHWCs which can give professional consultation service or recovery programs for GD patients now. 


\title{
SY5-1
}

\section{Why do people watch pornography? The motivational basis of (problematic) pornography use}

\author{
Beáta Böthe ${ }^{1 *}$, Nora Bella ${ }^{1}$, Istvan Toth-Kiraly ${ }^{1}$, Zsolt Demetrovics ${ }^{1}$, Gabor Orosz ${ }^{2}$ \\ ${ }^{1}$ Institute of Psychology, ELTE Eötvös Loránd University, Budapest, Hungary \\ ${ }^{2}$ Department of Psychology, Stanford University, Stanford, CA, USA \\ bothe.beata@ppk.elte.hu
}

Recently, more people has been viewing pornography and it has become more socially accepted than ever before. Problematic pornography use (PPU) and pornography use frequency (PUF) showed positive, but small-to-moderate associations in previous studies suggesting that these two characteristics of pornography use are related but yet may be considered as distinct domains of pornography use with potentially different backgrounds. Therefore, the question arised why some individuals become problematic users, while others do not engage in pornography use in a problematic manner despite high-frequency of use. Structural equation modeling (SEM) was employed to examine the associations of pornography use motivations, PPU and PUF $(\mathrm{N}=1,082$; females $=537,49.6 \%)$. According to the results of SEM $(\mathrm{CFI}=.942, \mathrm{TLI}=.935, \mathrm{RMSEA}=.064)$, PPU was positively and weakly related to sexual pleasure, fantasy, boredom avoidance and emotional avoidance motivations, whereas it was positively and moderately related to stress reduction motivation. PUF use was positively and weakly related to stress reduction and boredom avoidance motivations, and positively and moderately related to sexual pleasure motivation. The present results suggest that it might be important to take into account the motivational background of PPU and the PUF use to identify more subtle differences between high-frequency and problematic use.

\section{SY5-3}

\section{Masculine face preference predicts online sexual activities}

\author{
Marc N Potenza ${ }^{1 *}$, Lijun Chen ${ }^{2}$ \\ ${ }^{1}$ Yale University \\ ${ }^{2}$ School of Humanities and Social Sciences, Fuzhou University, China \\ qqkx159@126.com
}

\begin{abstract}
A large number of studies have revealed that stronger preferences of masculine male face imply the tendency of mate selection in short-term and may have less restricted social attitudes and sexual behavior. At the same time, unrestricted sociosexual orientation (SOI) is closely related to online pornography. In Study 1, we investigated the predictive effect of male facial masculinity on problematic online pornography use by using structural equation model. In addition, Condition-dependent Preference theory holds that self-attraction will affect mate selection strategies and facial preferences. In Study 2, we add self-attraction factors to expand the structural model of Study 1, and the whole model is validated in Gay man sample.
\end{abstract}


The main results and conclusions are as follows: (1) Masculine preference for male face can predict problematic Internet pornography use through the SOI, and sociosexuality work as a complete mediating effect. (5) In the gay men group, we found that the extended chain mediation model of self-attraction level $\rightarrow$ masculinity preference of male face $\rightarrow \mathrm{SOI} \rightarrow$ problematic online pornography use.

\title{
SY5-4
}

\section{Stress and neural responsivity to sexual explicit material (SEM) in men}

\author{
Rudolf Stark*, Sanja Klein, Charlotte Markert, Jana Strahler
}

Department of Psychotherapy and Systems Neuroscience, University of Giessen, Germany rudolf.stark@psychol.uni-giessen.de

Hypersexual behaviors, e.g. excessive pornography consumption are often interpreted in terms of behavioral addiction. While drug addiction research points to an association between stress and heightened addiction behavior, little is known about stress and hypersexual behaviors. This ongoing project investigates the impact of stress and individual pornography consumption on the neural processing of sexual explicit material (SEM) using an adaption of the Monetary Incentive Delay Paradigm. Participants (all male) undergo either a psychosocial stress test or a non-stress control condition followed by functional magnetic resonance imaging of alternating SEM anticipation and SEM consumption phases (Sexual Incentive Delay). Humoral, psycho-physiological, behavioral, and subjective data is also collected.

During an interim analysis ( $\mathrm{n}=56$ stress, $\mathrm{n}=56$ non-stress), SEM compared to control stimuli resulted in greater brain activity during both anticipatory and consummatory phases. They differentially recruited the putamen (anticipation) and amygdala, OFC and thalamus (consumption). Comparing the groups, the non-stress condition was associated with increased NAcc and caudate activity during anticipation while acute stress was associated with increased dorsolateral PFC activation during consumption.

The results of this interim analysis suggest an altered neural responsivity to SEM under stress, emphasizing the role of stress in hypersexual behaviors.

\section{SY5-5}

\section{The balancing role of insula during inhibitory-control in association with symptoms of problematic pornography use in heterosexual males}

\author{
Stephanie Antons*, Matthias Brand \\ University of Duisburg-Essen \\ stephanie.antons@uni-due.de
}

One core characteristic of problematic pornography use (PPU) is diminished control over the pornography use, especially when pornographic cues are present. Based on dual process 
models of addiction, such cues are assumed to cause an imbalance between the reflective (e.g. inferior frontal gyrus) and impulsive (e.g. ventral striatum) brain systems resulting in unregulated behavior. In addition, the insula plays a balancing role between these systems. The current study used a Stop-Signal task (SST) including neutral and pornographic images during fMRI as well as questionnaires to investigate associations between symptoms of PPU, craving, and neural representations of inhibitory-control. We examined 28 heterosexual males with varying symptom severity of PPU. Symptom severity of PPU and craving correlated positively with SST performance. SST performance was associated with insula and ventral striatum activity during inhibitory-control processing. Symptom severity of PPU was associated with insula and inferior frontal gyrus activity during pornographic image processing. The results imply that the insula plays a pivotal role in inhibitory-control when pornographic images are present. The higher involvement of insula in inhibitory-control and pornographic image processing might be an indicator for a higher need to balance between the reflective and impulsive brain systems in PPU.

\title{
SY6-1
}

\section{Impulsivity and Disordered Gambling: An Introduction and Update}

\author{
Samuel Chamberlain ${ }^{1 *}$, Jon Grant ${ }^{2}$, Masanori Isobe ${ }^{3}$ \\ ${ }^{1}$ University of Cambridge \\ ${ }^{2}$ University of Chicago \\ ${ }^{3}$ Kyoto University \\ srchamb@gmail.com
}

Though Gambling Disorder is currently listed as a Substance Use and Related Disorder in DSM-5, it was listed as an Impulse Control Disorder (ICD) in previous DSM versions, highlighting early recognition that impulsivity is central to its pathophysiology. In this presentation, I shall begin by defining impulsivity and consider how it can be fractionated not only at the level of symptoms but also using cognitive tests and questionnaires. I then move on to introduce core features of Gambling Disorder viz diagnostic criteria, and comorbidities (including ADHD and ICDs). Core Delphi-derived constructs central to the neuropsychological assessment of Gambling Disorder will be considered, in relation to impulsivity. I will then present as yet unpublished data from a meta-analysis of case-control studies exploring impulsivity in gambling disorder, using cognitive tests. Lastly, I present unpublished data regarding the role of impulsivity in the manifestation of empirically derived longitudinal subtypes of disordered gambling, and consider what role impulsivity may actual play in the disorder, when contrasted to compulsivity (vulnerability versus chronicity). 


\title{
SY6-2
}

\section{Neuroimaging of Gambling Disorder}

\author{
Masanori Isobe \\ Kyoto University, Department of Psychiatry \\ masaniso@kuhp.kyoto-u.ac.jp
}

Gambling disorder (GD) is a functionally impairing condition, which is recently classified in the category of substance-related and addictive disorders (DSM-5). In spite of implication of disease models as behavioral addictions, structural and functional abnormalities are still inconclusive and not consistently shared with the other addictions. For effective intervention of GD, biological marker should be indispensable, and imaging 'endophenotypes' can be one of the strongest candidates for ease of access. In order to establish the endophenotype of GD, it is necessary to address the influence of various factors, such as the possibility that diagnostic criterion do not have roots deep in biological abnormalities, cultural differences found in the background of GD, and so on. In the current presentation, we will report previous findings from neuroimaging studies conducted in my research group with regard to the relationship of the symptoms and behavioral characteristics of GD with the brain structure and function examined with MRI. We will also introduce structural abnormality involved in inhibitory control is common between the disease group and at risk group. Furthermore, the results of mega-analysis of structural MRI and cross-cultural work between Japan and the USA will also be exhibited to provide a provisional phenotype.

\section{SY6-3}

\section{Pharmacological Targets for Treatment of Gambling Disorder}

\author{
Jon E. Grant* \\ University of Chicago \\ jongrant@uchicago.edu
}

Background: There are no pharmacological interventions that are currently approved in any jurisdiction for the treatment of gambling disorder. Several medications, however, have been investigated as candidate treatments for gambling disorder. These have included serotonin reuptake inhibitors, lithium, glutamatergic agents, neuroleptics, and opioid antagonists.

Methods: In this talk we address current research related to pharmacological treatment of gambling disorder as well as predictors of treatment response.

Results: Recent research demonstrates that understanding subtypes of disordered gamblers, using imaging and neurocognition, may better focus pharmacological approaches to treatment.

Discussion: Treatments for gambling disorder may be effective but further refinement of pharmacotherapy may improve outcomes. 


\title{
SY7-1
}

\section{Criteria for other specified disorders due to addictive behaviors}

\author{
Matthias Brand ${ }^{*}$, Marc N. Potenza ${ }^{2}$ \\ ${ }^{1}$ University of Duisburg-Essen \\ ${ }^{2}$ Yale University School of Medicine \\ matthias.brand@uni-due.de
}

The ICD-11 includes gambling disorder and gaming disorder in the category of disorders due to addictive behaviors. The sub- category of "other specified disorders due to addictive behaviors" would benefit from precise definition. Some researchers argue that pornographyuse disorder, as a potential facet of compulsive sexual behaviors, and buying-shopping disorder might belong to this category, although these conditions are currently being proposed as impulse-control disorders. We summarize the literature on several types of behavioral addictions, including phenomena that are not yet recognized as clinical conditions, such as social-network-use disorder, and suggest criteria for subsuming disorders under the term of "other specified disorders due to addictive behaviors". Consistent with gaming-disorder criteria, scientific evidence should demonstrate that the phenomenon of interest is clinically relevant and associated with functional impairments in personal, family, social, educational, occupational, and other important life domains due to the addictive behavior. Second, the phenomenon of interest should be explained most appropriately by theories that describe the processes underlying addictive disorders; i.e., substance-use, gambling and gaming disorders. Third, multi-methodological studies should demonstrate parallels on psychological and neurobiological levels between substance-use disorders, gambling and gaming disorders, and the phenomenon of interest.

\section{SY7-2}

\section{Problematic pornography use: A compulsive sexual behavior disorder, an internet-use disorder and / or an other specified disorder due to addictive behaviors?}

\author{
Marc N. Potenza* \\ Yale University \\ marc.potenza@yale.edu
}

The internet has changed many aspects of day-to-day life. While psychiatric disorders related to internet use have been proposed, internet-related problematic behaviors have often been subsumed into existing diagnoses in widely used entities that define and classify mental health conditions (e.g., DSM-5, ICD-10). In ICD-11, the diagnostic entities of compulsive sexual behavior disorder and "other specified disorder due to addictive behaviors" have been introduced, and "predominantly offline" and "predominantly online" specifiers have been 
included for gaming and gambling disorders. Pornography viewing is a prevalent behavior online, and many individuals develop problems related to pornography viewing. As such, a question arises regarding how best to define and classify problematic pornography use. This presentation will review clinical, neurobiological and other data relating to problematic pornography use and consider issues of definition and classification.

\title{
SY7-3
}

\section{Buying-shopping disorder: Clinical and empirical evidence to support its inclusion in ICD-11}

\author{
Astrid Müller* \\ Hannover Medical School \\ mueller.astrid@mh-hannover.de
}

The aim of this talk is to address clinical and theoretical considerations as well as empirical evidence for buying-shopping disorder (BSD). BSD is characterized by excessive preoccupations with buying / shopping, poorly controlled impulses to purchase that are experienced as irresistible, and recurrent maladaptive buying / shopping excesses that lead to marked distress and impairments. Efforts to stop BSD episodes are unsuccessful, despite the awareness of repeated break-downs in self-regulation, experiences of post-purchase guilt and regret, comorbid psychiatric disorders, reduced quality of life, familial discord, work impairment, financial problems and other negative consequences. Population-based studies provided evidence that BSD is a cross-national problem with an estimated point prevalence of $5 \%$. In the ICD-11 coding tool, BSD is now mentioned as an example for the residual category "Other specified impulse control disorders" (category 6C7Y). Recent research findings, however, indicate shared key characteristics of BSD with substance-use disorders and gambling disorder such as maladaptive decision-making processes, increased salience of disorder-specific stimuli, cue-induced craving and reward seeking. The presentation will be offering a perspective to conceptualize BSD as a disorder due to addictive behaviors that should be examined in its genetic, psychological, social, cultural, and environmental contexts.

\section{SY7-4}

\section{Empirical evidence on prevalence and psychological mechanisms of problematic social network use}

\section{Hans-Jürgen Rumpf*}

University of Luebeck, Department of Psychiatry and Psychotherapy hans-juergen.rumpf@uksh.de

Background and aims: In ICD-11, besides gaming and gambling disorder there will be the opportunity to classify other specified disorders due to addictive behaviors. One of the areas 
discussed for this category relates to problematic social network use. Aim of the presentation is to review the current evidence in this area.

Methods: A narrative literature review was performed.

Results: Studies on prevalence of problematic social network use differ widely which is in part due to different measures and samples. Nevertheless, prevalence rates are high compared to gambling or gaming and higher for women than men. A number of co-occurring mental disorders have been found including depression, anxiety, ADHD, and OCD. In addition, fear of missing out (FOMO) and low self-esteem are associated with problematic social network use as well as neuroticism and conscientiousness in the area of personality traits. Findings on addictive mechanisms and cases seeking treatment exist.

Conclusions: Problematic social network use is an important area of research and a possible candidate for the classification of other disorders due to addictive behaviors. More sound studies are needed.

\title{
SY7-5
}

\section{Distinguishing Passion from Addiction is crucial to avoid pathologizing common behaviors}

\author{
Joël Billieux*, Maeva Flayelle \\ University of Luxembourg \\ joel.billieux@uni.lu
}

Gambling and gaming disorders have been proposed as "disorders due to addictive behaviors" in ICD-11. In addition to these conditions, the designation "other specified disorders due to addictive behaviors" (currently coded "6C5Y" in ICD-11) was considered. This descriptor reflects the view that other specific excessive or problematic behaviors that may be considered as disorders due to addictive behaviors (beyond gambling and gaming) deserve attention.

Increasing evidence supports that intense involvement in appetitive activities is not essentially problematic nor necessarily associated with negatives consequences and functional impairment, suggesting the existence of behavioral patterns that fall within a heavy - but unproblematic - engagement. Furthermore, when specifically applied to behavioral addictions, some traditional addiction criteria reflect peripheral features that are not necessarily indicative of pathology, whereas others reflect core features that can be used to adequately identify pathological behavior.

In such a context, our objective is to clarify what are the respective characteristics of high involvement versus pathological involvement in popular appetitive activities (such as social networking, pornography consumption, or TV series watching), where are the boundaries between them, and how implementing screening and diagnostic tools that allow for distinguishing the two. 
SY8-1

\title{
Survey of the relationship between Internet gaming disorders and cerebral blood flow
}

\author{
Tomohiro Shirasaka ${ }^{1 *}$, Miyuki Tsuneta ${ }^{1}$, Hisakazu Kimura ${ }^{1}$, Toshikazu Saito ${ }^{2}$ \\ ${ }^{1}$ Department of Psychiatry, Teine Keijinkai Hospital, Sapporo, Japan \\ ${ }^{2}$ Miki Mental Clinic, Sapporo, Japan \\ shirasaka.t@gmail.com
}

The Internet was originally designed to research activities. However, there has been an increase in the use of the Internet for entertainment including video games. Internet gaming disorders and related behaviors have been attracting the attention of mental health researchers, although this field is still in its infancy. There are some studies that Internet gaming disorders might cause the same brain changes that are seen in alcoholics and drug addicts. Social isolation has increasingly become a problem in Japan and has been hypothesized to be related to Internet gaming disorders. Particularly amongst students, Internet gaming disorders may be a major factor in social withdrawal. We conducted a survey about social isolation of Internet gaming disorders and relating cerebral blood flow (CBF) by the RI scintigraphy.

Method: Subjects were 13 Internet gaming disorders and 15 healthy controls. To evaluate regional patterns of $\mathrm{CBF}, \mathrm{Z}$-score analysis was carried. To evaluate regional patterns of CBF alterations, two-sample t-test values were calculated on a pixel- by-pixel basis between Internet gaming disorders and to normal control.

Results: Compare with the normal control, we found Internet gaming disorders have a significant reduction of the blood flow at the frontal lobe. We will show the detail in this presentation.

\section{SY8-2}

\section{Structural and functional brain alterations related to psychological factors in excessive use of digital devices}

\section{Dai-Jin kim*}

Department of Psychiatry, Seoul St. Mary's Hospital, The Catholic University of Korea, College of Medicine kdj922@catholic.ac.kr

Problems of digital device including Internet gaming disorder (IGD) and problematic smartphone use (PSU) have been raised because of the loss of control over Internet use, represented as the persistence of online gaming and smartphone use despite the awareness that it is directly harmful to one's psychosocial performance.

Functional and structural MRI data were acquired with a 3-Tesla MAGNETOM Verio system. The IGD group was selected on the basis of the clinician-administered interview and diagnostic criteria of IGD in the DSM-5, and PSU group was estimated with the Korean Smartphone Addiction Proneness Scale. 
We found lower gray matter (GM) density in the left dorsolateral prefrontal cortex in the IGD, and the GM density was associated with lifetime usage of Internet gaming, depressed mood, craving, and impulsivity in IGD. In addition, we observed lower functional connectivity between the right OFC and NAcc, and between the left OFC and MCC, and we found that more severe withdrawal symptoms were associated with higher depressive symptom in PSU.

These studies examine the evidence for brain alteration related to excessive use of digital devices and provide an important opportunity to advance our knowledge of problematic internet use.

\title{
SY8-3
}

\section{How can therapy animals help patients in Internet Addiction treatment?}

\author{
TaeKyung Lee ${ }^{I^{*}}$, Jangrae Kim², Jungwon Choi ${ }^{3}$ \\ ${ }^{1}$ ChunCheon National Hospital, ChunCheon City, Gangwon Province, South Korea \\ ${ }^{2}$ National Medical Center, South Korea \\ ${ }^{3}$ National Center for Mental Health, South Korea \\ TKLeeMD@gmail.com
}

Internet Addiction (IA) is a growing social issue which is being debated worldwide. However, breaking an addiction is one of the hardest things you can do. While IA is increasingly prevalent, treatment modalities have ignored the unique aspects of this particular process addiction. IA has been known to be positively associated with social anxiety and discontent with peer interactions, and with parenting attitudes, family communication, and family cohesion. That's why the support of your loved ones is so necessary. But what about the help of animal companions? If you're an animal lover, you're probably well aware of the uplifting effects pets have on your life. And when applied to addiction treatment, these can have even farther-reaching impacts than you might expect. In this session, we will present our experience of animal-assisted therapy in IA treatment.

\section{SY8-4}

\section{Internet addiction and internet gaming disorder: the comorbidity and associated factors}

\section{Chih-Hung Ko*}

Department of Psychiatry, Kaohsiung Medical University Hospital chihhungko@gmail.com

Internet addiction and internet gaming disorder are important issues of mental health in modern society, particularly for adolescents. These two problems were noted to be prevalent in Asia area both in adolescents and young adults. Comorbidity is an essential issue when 
treating adolescents with Internet addiction. Intervention for comorbidity usually benefits for treatment of internet addiction. ADHD, depressive disorder, general anxiety disorder, and social phobia had been repeatedly reported to associate with internet addiction. However, why these disorders comorbid with internet addiction had not well understood. These presentations pay attention to the comorbidity of internet addiction and associated factors of these comorbid disorders. We also provided the clinical experience in evaluation and intervention for these comorbid disorders of internet addiction.

\title{
SY9-1
}

\section{Mechanism of Stereotyped Behaviors in Autism Spectrum Disorder from Philosophical Perspective}

\section{Tsubasa Aikawa*}

Musashi High School \& Junior High School

t_aikawa_fr@akane.waseda.jp

Stereotyped behaviors in ASD seem meaningless at a glance, but they play an important role in making these patients feel comfortable and providing the mental and physical stability. Although inability to control stereotyped behaviors is considered as "dysfunction", it is, from a philosophical perspective, humanly primitive and "normal" to obtain comfort from repetitive behaviors. In the support of the ASD patients in education and welfare, an approach accepting, instead of denying and preventing, stereotyped behaviors as inevitable for one's mental and physical stability has generally been adopted.

On the other hand, gambling behaviors such as pachinko and slots have a self-therapeutic aspect which reduces psychological distress because they also bring comfort by repetition of the same pattern and simple and clear stimuli. This has a strong association with stereotyped behaviors of ASD in terms of inner experience. The philosophical interpretation of stereotyped behaviors in ASD supports the self-medication hypothesis for addictive behaviors and helps to understand the inner experience of patients with gambling disorder.

\section{SY9-2}

\section{Assistance in Gambling Disorder from Perspective of Regal Counselor}

\author{
Atsushi Inamura* \\ Inamura Atsushi Judicial Scrivener Office, Legal Counselor \\ PXX02744@nifty.com
}

Gambling disorder causes several problems: financial problems such as debt; domestic problems such as family discord; professional problems such as unemployment; maladaptation problems such as withdrawal from society, etc. These problems are independent of each other because of the results of, or the factors caused by, the gambling disorder, or both. 
For nearly twenty years, the author has been supporting sufferers of gambling disorder and counseling the whole of life support based on debt settlement. As a result of these long years of service, our assistance has led to finding a permanent solution to their gambling disorder. The author believes that the key to recovery is improvement of self-esteem of the sufferer.

Merits of legal counseling: The repayment of debts is the greatest worry of the sufferer and their family. Our legal experts, who can intervene and facilitate debt settlement in Japan, are the social resources - the earlier our experts intervene, the better it is for debt settlement. It can be said that seeking debts is a symptom of an underlying gambling disorder in a person. Our legal experts are trained to understand the background of the debt and identify whether there is an underlying gambling disorder.

SY9-3

\title{
The characteristics of patients with both gambling disorder and neurodevelopmental disorders in a clinical setting
}

\author{
Masuo Tanaka*, Takashi Hashimoto
}

Koryo Hospital

masuo926@hotmail.com

We start to treat gambling disorder 18 years ago. Lately, telephone and / or direct consultations about gambling disorder have been increasing at our setting, which is only one for treatment of addiction in our medical area. In addition, medical consultations about neurodevelopmental disorders and comorbidities of both neurodevelopmental disorders and gambling disorder also have been increasing for several years at our setting.

In two years, fifty-four patients with gambling disorder visited in our hospital to treat it. In previous study, although we suggested that the ratio of inpatients with both alcohol use disorder and attention deficit hyperactivity disorder (ADHD) was about $25 \%$, that of both gambling disorder and ADHD was 29.6\% (16 / 54). In addition, that of both gambling disorder and autism spectrum disorder, including mild cases, was $16 \%(9 / 54)$. The patients with both gambling disorder and ADHD committed more crimes about money than those who have only gambling disorder. $(X=5.41, \mathrm{p} 0.01)$ 
SY9-4

\title{
Stress Management of Persons with Gambling Disorder Using the Wechsler Adult Intelligence Scale
}

\author{
Taku Sato* \\ Naruse Mental Clinic, psychiatrist \\ naruse-cl@royal.ocn.ne.jp
}

We are disposed to maintain internal environment in a state of constant homeostasis, and anything that disturbs this is called a stressor. Analysis regarding what kind of stressors we are at risk of receiving if the stress reaction attempting restore the internal environment disturbed by the stressor becomes a gambling disorder is effective information for the treatment of gambling disorder. The Wechsler Adult Intelligence Scale (WAIS-III) is a general test to measure standardized intelligence quotient (IQ) in adults over the age of 16 and is not limited to gambling-related issues. In this examination, it is possible to evaluate not only overall IQ, but also the four group indices of verbal comprehension, perceptual reasoning, working memory, and processing speed. In the present study, based on the group index variance of the Wechsler Adult Intelligence Scale, we analyzed the stressors of persons with gambling disorder and devised an intervention method that may be effective for reducing the risk of gambling and improving individual self-esteem.

\section{SY10-1}

\section{The development of a risk assessment tool for paraphilic sex offenders: The Japanese version of the Static-99}

\author{
Takayuki Harada ${ }^{1 *}$, Kazutaka Nomura ${ }^{2}$ \\ ${ }^{1}$ University of Tsukuba \\ ${ }^{2}$ Waseda University \\ tkharada77@gmail.com
}

The Japanese version of the Static-99 was developed to assess recidivism risk of sex offenders with paraphilic disorders. The participants were male 167 paraphilic sex offenders under treatment. Their recidivism was observed for one year. Of all 167 participants, 8 participants (4.8\%) were categorized as "low risk", 44 (26.3\%) were "low-medium", 87 (52.1\%) were "medium-high" and 28 (16.8\%) were "high". The demographics across the risk levels were compared. The significant difference were not found except the marital status and the employment. In terms of problem behavior, frotteurism in the low risk was significantly less and significantly more the in med-high risk. Pedophilia was significantly more in the low-med risk group. In summary, the higher risk participants were characterized by being single and not employed and frotteurism involvement. The overall recidivism rate for 167 participants was $10.8 \%$ (18 of 167). No one in the low risk level reoffended $(0 / 8)$ but 10 of $28(35.7 \%)$ of the high-risk participants recidivated. The relapse rates across the risk category were sig- 
nificantly different $(\chi 2(3)=22.21, \mathrm{p}=<0.001)$ and the residual analysis indicated that the proportion of relapsed high-risk participants was significantly high. The AUC was calculated and the value of $0.77(95 \% \mathrm{CI}=0.63$ to 0.89$)$ was obtained.

\title{
SY10-2
}

\section{Sexual offender assessment and treatment system in Japanese correctional institutions}

\author{
Takayuki Harada ${ }^{*}$, Makiko Asahina $^{2}$, Kazutaka Nomura ${ }^{3}$, Yuki Tanaka ${ }^{3}$ \\ ${ }^{1}$ Tsukuba University \\ ${ }^{2}$ Ministry of Justice, Japan \\ ${ }^{3}$ Waseda University \\ mhsep@yahoo.co.jp
}

Japanese government has introduced integrated sexual offender assessment and treatment initiatives into criminal justice system in 2007. How and what types of sexual offenders are identified as the subjects of the program, and how they are treated in correctional institutions will be presented. Since the addiction model is not explicitly utilized as the primary framework for understanding sexual offenders in the program, the probable explanations will be made. Potential advantages and disadvantages of introducing the addiction model into the program will be discussed.

\section{SY10-3}

\section{The influence of empathic victim intervention on the risk of reoffending by sex offenders}

\section{Kazutaka Nomura*}

Faculty of Human Sciences, Waseda University

k-nomura@aoni.waseda.jp

The treatment of sex offenders continues to be based on the premise that a lack of empathy increases the risk of recidivism. On the other hand, empirical studies have not found any repeat offense prevention effect from such efforts, and some studies suggest that it will even cause repeat offenses. In this presentation, we report the results of empirically examining the influence of empathic victim intervention on sex offenders from the perspective of cognitive behavioral therapy. The results of a series of studies show that sex offenders responded with a positive affect to a script presenting a sexual victim scenario. Empathic victim intervention has an increased positive affect and has been shown to enhance the risk of sexual offense. Furthermore, it was suggested that the increased risk of sexual criminal behavior could be reduced by implementing psychological interventions that reduce emotional responses. For this reason, it is thought that the implementation of empathic victim intervention needs to be considered as being a relevant link between emotional response and recidivism risk. 
SY10-4

\title{
Practicing cognitive behavior group therapy for sexual offenders using a rolling format in the medical institutions of Japan
}

\author{
Yuki Tanaka* \\ Graduate School of Human Sciences, Waseda University \\ hautbois@fuji.waseda.jp
}

For community-based treatment in medical institutions, it is not realistic to provide cognitive behavior therapy individually for each of the sexual offenders. Therefore, cognitive behavior group therapy (CBGT) is the main model of treatment. Among the formats of CBGT, closed format and open format are considered to be the main formats. In the closed format, members are fixed, gradual intervention is possible, and group cohesiveness gradually increases, but is not flexible in terms of participation. Although the open format has high flexibility of participation, it is difficult to increase group cohesiveness since participants are frequently replaced. On the other hand, the rolling format is advantageous because members can participate at any time, and they can proceed with programs in their progressive groups (Ware \& Bright, 2008). Therefore, rolling format is considered to be the most appropriate method to treat sexual offenders. In this symposium, the practice of CBGT, using a rolling format, as a community-based treatment for sexual offenders in Japan will be discussed.

\section{SY11-1}

\section{An overview of NAMS services especially pertaining to behavioural disorders}

\section{Lee Cheng*}

Institute of Mental Health, NAMS

cheng_lee@imh.com.sg

The National Addictions Management Service (NAMS) was formed in 2008 with the support of the Ministry of Health, Singapore to provide a national treatment focus and direction for the management of addiction disorders (both substance and behavioural addictions). Beyond clinical services such as the Helpline and the clinic consultations, NAMS is also active in community outreach, public education and outpatient group programmes.

NAMS is located at the Institute of Mental Health and is managed by a dedicated team of multi-disciplinary staff who will conduct a thorough assessment and devise a personalized management for each client.

This introductory talk will provide an overview of NAMS services especially pertaining to behavioural disorders. 
SY11-2

\title{
An overview of the medical management model for gambling and Internet-related disorders
}

Zhang Weibin Melvyn*

Institute of Mental Health, NAMS

Melvyn_WB_ZHANG@imh.com.sg

Abstract for speaker ${ }^{2}$

Dr Zhang Weibin Melvyn, Associate Consultant (NAMS)

Gambling and Internet-related disorders are some of the common behavioural disorders seen and managed at the National Addictions Management Service.

In this symposium, an overview of the medical management model for gambling and Internet-related disorders will be provided.

In addition, pertinent research studies that have been previously undertaken by NAMS will also be presented. These studies cover the epidemiology of gambling disorders in Singapore, the use of innovative web-based technologies to reach out to individuals with gambling disorders, and relevant epidemiological studies done for that of Internet Gaming Disorder.

\section{SY11-3}

\section{The application of a behavioural intervention approach, Choice Theory / Reality Therapy, in calibrating gaming into a more adaptive form as part of quality living}

\author{
Alvin Wah Loong Cheong* \\ Institute of Mental Health, NAMS \\ Alvin_Wl_CHEONG@imh.com.sg
}

Two common types of behavioural addictions are seen in NAMS - gambling and gaming. The latter is fast growing in the city state of Singapore, and is of great concern among parents and educators. This presentation shares about the application of a behavioural intervention approach, Choice Theory / Reality Therapy, in calibrating gaming into a more adaptive form as part of quality living. 
SY11-4

\title{
Collaboration, training and outreach initiatives that NAMS has with community partners and persons in recovery
}

\author{
Tan Jian Hui Clement ${ }^{*}$ \\ Institute of Mental Health, NAMS \\ clement_jh_tan@imh.com.sg
}

Community support plays an essential support towards the recovery for a person with addiction.

This presentation shares about the collaboration, training and outreach initiatives that NAMS has with community partners and persons in recovery.

\section{SY12-1}

\section{On Facebook usage, Facebook use disorder and personality}

\section{Cornelia Sindermann*, Christian Montag}

Department of Molecular Psychology, Institute of Psychology and Education, Ulm University, 89081 Ulm, Germany

cornelia.sindermann@uni-ulm.de

Background: The present work aimed to understand whether Facebook-users (FUs) and non-users (FNUs) differ in sociodemographic variables and / or the Big Five personality traits and their sub-facets. Additionally, we investigated whether the Big Five and their subfacets are associated with tendencies towards Facebook Use Disorder (FUD).

Methods: $3835(\mathrm{n}=2366$ males) participants $(\mathrm{M}($ age $)=32.18, \mathrm{SD}=11.82)$ between 11 and 75 years of age filled in the Big Five Inventory as well as one item about whether they have a Facebook account (FU / FNU). FUs also filled in a FUD scale. Results: FUs were younger, higher educated and the gender ratio of males to females was smaller as compared to the FNU group. Moreover, FUs scored higher in extraversion (complete scale, $\mathrm{p}=$ .002), whereas FNUs scored higher in conscientiousness and both of its sub-facets order and self-discipline ( $p$-values $<.001)$. Tendencies towards FUD were especially negatively correlated with conscientiousness $(\rho=-.12, \mathrm{p}<.001)$ and its sub-facet self-discipline $(\rho=-.15$, $\mathrm{p}<.001)$, and positively with neuroticism $(\rho=.17, \mathrm{p}<.001)$ and both of its sub-facets (anxiety: $\rho=.15, p<.001$; depression: $\rho=.17, p<.001)$. Conclusion: The observed differences in sociodemographic variables and personality need to be considered when evaluating results carried out via Facebook. The association between personality and tendencies towards FUD is strengthened. 
SY12-2

\title{
The Associations between Problematic Social Networking Site Use and Psychopathology
}

\author{
Zaheer Hussain ${ }^{1 *}$, Mark D. Griffiths ${ }^{2}$ \\ ${ }^{1}$ University of Derby \\ ${ }^{2}$ Nottingham Trent University \\ z.hussain@derby.ac.uk
}

Background and Aims: Research has shown a potential association between problematic social networking sites (SNSs) use and psychiatric disorders. The primary objective of this paper was to review and identify studies examining the association between problematic SNS use (PSNSU) and comorbid psychiatric disorders.

Sampling and Methods: A literature search was conducted using several databases. Problematic SNS use (PSNSU) and its synonyms were included in the search. Information was extracted based on problematic SNS use and psychiatric disorders, including attention deficit and hyperactivity disorder (ADHD), obsessive compulsive disorder (OCD), depression, anxiety, and stress. A total of nine studies met the predetermined inclusion and exclusion criteria.

Results: The findings of the systematic review demonstrated that most research has been conducted in Europe and all comprised cross-sectional survey designs. In eight (of the nine) studies, problematic SNS use was correlated with psychiatric disorder symptoms. Of the nine studies (some of which examined more than one psychiatric symptom), there was a positive association between PSNSU and depression (seven studies), anxiety (six studies), stress (two studies), ADHD (one study), and OCD (one study).

Conclusions: Overall, the studies reviewed showed associations between PSNSU and psychiatric disorder symptoms. Most associations were found between PSNSU, depression, and anxiety.

\section{SY12-3}

\section{Facebook use as a possibility of stress reduction and the relevance of Internet-communication disorder}

\author{
Elisa Wegmann*, Matthias Brand \\ General Psychology: Cognition and Center for Behavioral Addiction Research (CeBAR), \\ University of Duisburg-Essen, Germany \\ elisa.wegmann@uni-due.de
}

Social-networking-sites (SNS) are online-communication services, often used for distraction and procrastination. Based on the definition of gaming-disorder in the ICD-11, Internetcommunication-disorder describes the uncontrolled use of SNS. Researchers discuss whether the SNS use is associated with stress reduction and whether dysfunctional use affects stress 
management. In an experimental setting, 105 participants underwent a stress-induction procedure and were randomly assigned to one of three stress-reduction conditions: Facebook browsing, analogous magazine browsing, or waiting. State-Trait-Anxiety- Inventory was administered repeatedly to assess alterations in stress experience. Tendencies of pathological SNS use were measured using a modified version of the short Internet-Addiction-Test. The results illustrate that after stress induction, the subjective stress level was significantly lower in both the Facebook-browsing and magazine-browsing conditions compared to the waiting condition. Impairments in the ability to reduce stress was negatively associated with the symptom severity of a pathological SNS use. The results emphasize that browsing through analogous magazines and SNS can similarly reduce subjective stress. Stress management seems to be impaired in users with higher symptom severity, especially after confrontation with specific cues. Future studies should investigate causes of this deficit in individuals with Internet-communication-disorder.

\title{
SY12-4
}

\section{A path model linking preoccupied attachment style and virtual social support to social networking site addiction among college students: positive outcome expectancy as mediator and actual social support as moderator}

\author{
Huei-Chen $\mathrm{Ko}^{2 *}$, Lin-Chieh Hung ${ }^{1}$, Cheng-Chieh $\mathrm{Li}^{3}$ \\ ${ }^{1}$ Asia University \\ ${ }^{2}$ Asia University, China Medical University \\ ${ }^{3}$ National Cheng Kung University \\ jennyko@asia.edu.tw
}

Objectives: Previous studies showed internet use positive outcome expectancy mediated the relationship of personality and of higher virtual social support to IA. The actual social support moderated the association of cognitive expectations with addictive behaviors. We hypothesized the mediating effect of the social networking site (SNS) positive outcome expectancy on linking both of the preoccupied attachment and of virtual social support to SNS addiction, which were moderated by actual social support. Methods: 1277 ( $\mathrm{n}=729$ females) college students completed a battery of questionnaires. Results: The structural equation model results showed both the preoccupied attachment and virtual social support directly and indirectly predicted SNS addiction mediated through SNS positive outcome expectancy. However, actual social support moderated both of the mediating pathways. Further analyses revealed that the link of SSN positive outcome expectancy with SNS addiction became weak when actual social support increased. Conclusions: Results supported that SNS positive outcome expectancy may play the role of mediating in the relationships of the preoccupied attachment and of virtual social support to SNS addiction. However, an increased in actual social support may protect the effect of SSN positive outcome expectancy on SSN addiction. The further studies should examine these hypotheses by gender and in a longitudinal design. 


\title{
SY12-5
}

\section{Understanding WeChat usage: a review on the existing literature}

\author{
Christian Montag* \\ Ulm University and University of Electronic Science and Technology of China \\ christian.montag@uni-ulm.de
}

Background: WeChat represents one of the most often used applications in Asian countries. Currently, over one billion WeChat users have been reported. The multipurpose application WeChat is not only used as a tool to communicate with others, but also to get information on daily life issues and to pay for services / products in manifold situations.

Methods: The background for this presentation at the ICBA in Yokohama / Japan is a literature review, which highlights several lines of different research avenues to understand WeChat usage.

Results: In detail, three research topics will be discussed which also have been summarized in a recent article by Montag, Becker \& Gan (2018) published in Frontiers in Psychology. These are i) motivational aspects of WeChat usage, ii) well-being and WeChat usage and iii) a societal perspective on WeChat usage.

Discussion and Outlook: Beyond a summary of the literature, it will be highlighted in what areas research on WeChat is still lacking and how neuroscientific research can give additional important insights into various aspects to understand individual differences in WeChat usage.

\section{SY13-1}

\section{The role of structural video-game characteristics in Internet gaming disorder severity}

\author{
Florian Rehbein ${ }^{*}$ \\ Criminological Research Institute of Lower Saxony, Hanover, Germany \\ florian.rehbein@kfn.de
}

Internet Gaming Disorder (IGD) has been included as a clinical condition warranting further study in the DSM-5 and will be included as Gaming Disorder in the category disorders due to substance use or addictive disorder in the upcoming edition of the International Classification of Diseases (ICD-11). Thus, for the first time Gaming Disorder will be officially recognized as behavioral addiction along with gambling disorder and videogames will have to be conceived as addiction-inducing facilities. Considering our knowledge about the etiology of addictive disorders in general, personal, social and product related risk factors could be relevant for gaming disorder. Studies have shown that particularly online role-playing games but also online shooter games more often lead to higher symptoms of Gaming Disorder. It could be assumed that particular structural characteristics could elevate the risk potential in certain games. However, research addressing the specific role of structural video game 
characteristics in IGD symptomatology is still scarce. The talk will give an overview on prior empirical studies addressing structural characteristics in games. It will be discussed, that the role of structural video-game characteristics is not only critical for our understanding of the nature of IGD but could also inform effective structural prevention measures.

\title{
SY13-2
}

\section{Screening tools for gaming disorder: What, how, why?}

Daniel L King*

School of Psychology, The University of Adelaide

daniel.king@adelaide.edu.au

Deciding on what measure to use to screen for gaming disorder has never been more difficult, from the perspective of choice. Over the last decade, and particularly since the inclusion of Internet gaming disorder in the DSM-5 in 2013, new scales for gaming disorder as well as problematic video gaming have been developed at an increasing rate. While many of these tools appear to be quite similar on face value, there are in fact several important considerations for researchers and clinicians to bear in mind when choosing a tool. This presentation will provide a critical summary of all available tools for screening for gaming disorder, which includes more than 20 tools designed to assess gaming-related problems, in addition to other more general Internet use-related tools. This talk will also consider some of the research and practical implications of the ICD-11 category of gaming disorder (GD) and the proposed WHO screening tool for gaming disorder.

\section{SY13-3}

\section{Prevalence of gaming disorder in China using the Chinese Gaming Disorder Brief Screening Questionnaire based on DSM-5 and ICD-11}

\author{
Wei Hao*, Ting Fang, Qijian Deng, Xiaojie Zhang, Ming Yuan \\ The Second Xiangya Hospital of Central South University \\ fangtingxy@163.com
}

Introduction: Gaming disorder is listed under "disorders due to addictive behaviors" in the ICD-11. The aims of this pilot study were to estimate and compare the prevalence of internet gaming disorder based on DSM-5 recommendations and ICD-11 criteria, respectively. Additionally, investigated the patterns of internet gaming habits in Chinese.

Methods: A total of 1334 participants were recruited from 30 provinces in China through website. IGD was assessed with the Chinese Gaming Disorder Brief Screening Questionnaire, a standardized questionnaire with all criteria of IGD in ICD-11 and included all nine criteria of IGD in DSM-5. 
Results: The prevalence rates of IGD were found to be around 9.2\% [95\% confidence interval $(\mathrm{CI})=7.7,10.8]$ by ICD-11 and $6.8 \%[5.5,8.2]$ by DSM-5. The average time spent gaming per day are most relevant variables for predicting GD. Boys and those who get higher scores in Barratt impulsiveness scale were significantly get higher risk for GD.

Conclusions: Four criteria from DSM-5 are not included in ICD-11 ('craving', 'tolerance', 'withdrawal' and 'immersion'), and more cautious should be paid to the clinicians when using ICD-11 for diagnosing GD.

\title{
SY13-4
}

\section{A cognitive behavioral group therapy program for adolescents with Internet Gaming and Internet Use Disorder: Effects at 12-months follow-up}

\author{
Carolin Szász-Janocha ${ }^{1 *}$, Eva Vonderlin ${ }^{1}$, Katajun Lindenberg ${ }^{2}$ \\ ${ }^{1}$ Institute of Psychology, Heidelberg University, Germany \\ ${ }^{2}$ Institute for Psychology, University of Education, Heidelberg, Germany \\ carolin.szasz@uni-heidelberg.de
}

Background: Facing the rising number of adolescents suffering from internet-related disorders and the growing research base on this phenomenon, the World Health Organization announced the inclusion of "Gaming Disorder" in the 11th version of the International Classification of Diseases. Despite the increasing number of studies evaluating treatment programs, evidence remains limited given the deficient quality of the evaluation studies. In this study, we examine the long-term effects of our 4-session early intervention program PROTECT + for adolescents with Internet Gaming Disorder (IGD) and Internet Use Disorder (IUD).

Methods: $\mathrm{N}=54$ adolescents, aged 9 to 19 years $(\mathrm{M}=13.48, \mathrm{SD}=1.72)$, participated in the PROTECT + group therapy program from April 2016 to December 2017. Effects on IGD and IUD symptom severity were assessed at baseline, at the end of the intervention, 4 and 12 months after admission via self-report and parental ratings.

Results: Multilevel models revealed a significant improvement in IGD and IUD pathology after 12 months $(\mathrm{p}<.001$ to $\mathrm{p}=004)$. Patients showed medium to high reductions in IGD and IUD symptom severity at 12 months follow-up $(d=0.73$ to $d=1.02)$.

Conclusions: The present study shows promising long-term effects of PROTECT + on IGD and IUD symptom severity. Randomized controlled studies are needed to confirm the present results. 


\title{
SY14-1
}

\section{The actual situation of the gambling industry and treatment systems of gambling disorder in Japan}

\author{
Moritoshi Kido ${ }^{1 *}$, Tatsuya Noda ${ }^{2}$, Nobuaki Takahashi ${ }^{3}$ \\ ${ }^{1}$ Osaka University of Commerce \\ ${ }^{2}$ Nara Medical University \\ ${ }^{3}$ Bukkyo University \\ kido79@daishodai.ac.jp
}

In Japan, according to the results of the national survey administered in 2017 by Ministry of Health, Labor and Welfare, the lifetime prevalence rate of gambling disorder went up to 3.6\% and past-year gambling disorder rate went up to $0.8 \%$. In this situation, the government legalized casino gambling and promotes the IR. At the same time, Japanese government made BASIC LAW ON MEASURES AGAINST GAMBING ADDICTION and local government due to administering the countermeasure of prevention and treatment. In this presentation we introduce the actual situation of Japanese gambling industry and the movement of casino promotions. Then we introduce the local government countermeasures, especially the local treatment system of gambling disorder and discuss about the future prospects of gambling disorder's treatment system of Japan.

\section{SY14-2}

\section{The efficacy of group cognitive behavioral intervention for pathological addiction to pachinko / pachislot}

\author{
Eiichi Kamimura* \\ Niigata University \\ kamimura@ed.niigata-u.ac.jp
}

To confirm the efficacy of group cognitive behavior intervention for problematic-gambling on Japanese pachinko / pachislot addicted adults, thirty-three volunteers were participated in 5 -session group therapy. The program consisted of self-monitoring of craving and gambling behaviors, identifying triggers, listing alternate activities, cue-exposure, and restructuring of excusing thoughts for gambling. Results revealed significant effects in reducing and ceasing of addictive behaviors. The author would propose the typology concerning intervention based on several functions; to stabilize one's mood, to avoid interpersonal interactions, to feel win something of value and to be absorbed in playing game-machines. 


\title{
SY14-3
}

\section{The development of the mobile version of the gambling craving coping application}

\author{
Kengo Yokomitsu* \\ College of Comprehensive Psychology, Ritsumeikan University \\ k-yoko@fc.ritsumei.ac.jp
}

In this presentation, I would summarize the research in relation to online and mobile intervention for gambling, alcohol, and drugs (e.g. Giroux et al., 2017) and introduce the Mobile version of the Gambling craving coping Application (MGA) that I have developed with a collaborator. MGA includes the following functions for disordered gamblers: (a) escape stimulus which could generate craving for gambling, (b) provide functional tips to cope with that craving, (c) acquire the coping skill for that craving. Finally, I would make opinions the ecological momentary intervention for disordered gamblers, such as a MGA.

\section{SY15-1}

\section{High-Frequency Pornography Users Are Not Always Problematic Users}

\author{
Beáta Böthe ${ }^{l^{*}}$, Istvan Toth-Kiraly ${ }^{1}$, Marc N. Potenza ${ }^{2}$, Gabor Orosz ${ }^{3}$, Zsolt Demetrovics ${ }^{1}$
}

\author{
${ }^{1}$ Institute of Psychology, ELTE Eotvos Lorand University \\ ${ }^{2}$ Yale University, School of Medicine \\ ${ }^{3}$ Department of Psychology, Stanford University \\ bothe.beata@ppk.elte.hu
}

\begin{abstract}
Although scarce in the literature, person-centered studies could answer questions regarding whether there are individuals who use pornography frequently and experience related problems and other individuals who use pornography just as frequently without experiencing negative consequences. The aims of the present study were to identify profiles of pornography users based on pornography use frequency (PUF) and problematic pornography use (PPU) by applying a person-centered analytic approach, and to examine whether the identified profiles could be distinguished based on theoretically relevant demographic and psychological constructs. Across three studies $(\mathrm{N}=14,006, \mathrm{~N}=483$, and $\mathrm{N}=672$, respectively), three distinct pornography- use profiles emerged: non-problematic low-frequency pornography users (68-73\% of users), non-problematic high-frequency pornography users (19-29\% of users), and problematic high-frequency users (3-8\% of users). Non-problematic and problematic high-frequency users showed notable differences in several constructs (e.g., depressive symptoms, boredom susceptibility, self- esteem, and basic psychological needs). Consequently, PUF cannot be considered as a sufficient indicator of PPU in itself as the number of non-problematic high-frequency users were 3-6 times higher than the number of problematic high-frequency users. Different psychological mechanisms may lay behind PUF with or without PPU, with findings having implications for interventions.
\end{abstract}


SY15-3

\title{
Moral Incongruence and Self-Reported Problematic Pornography Use: Enhancing Diagnostic Precision by Disentangling Beliefs and Behaviors
}

\author{
Joshua B Grubbs ${ }^{1 *}$, Rory C. Reid ${ }^{2}$, Samuel L. Perry \\ ${ }^{1}$ Department of Psychology, Bowling Green State University, Bowling Green, OH, USA \\ ${ }^{2}$ University of California, Los Angeles, California, USA \\ ${ }^{3}$ Department of Sociology, University of Oklahoma, Norman, Oklahoma, USA \\ GrubbsJ@BGSU.edu
}

With the recent inclusion of the Compulsive Sexual Behavior Disorder diagnosis in the ICD11 , there is increased interest in and scrutiny of compulsive sexual behavior patterns. One key area in which this scrutiny has emerged has been in the effects of personal morality and belief on people's interpretations of their own sexual behaviors. Using data from two U.S. nationally representative samples and a longitudinal sample of internet users, we demonstrate that moral incongruence regarding pornography use (e.g., using pornography, even when one thinks it is morally wrong) consistently moderates the associations between pornography use and self-reported feelings of addiction to pornography, and that the correspondence of moral incongruence and self-reported feelings of addiction continues over time. Findings generally indicate that pornography use is only very weakly associated with self-reported feelings of addiction or dysregulation at low levels of moral incongruence, but very strongly related to feelings of addiction or dysregulation at higher levels of incongruence. Such findings suggest that moral incongruence is an important consideration in the diagnosis and treatment of selfreported problems with compulsive sexual behaviors.

\section{SY15-4}

\section{Theoretical considerations of problematic pornography use}

\author{
Matthias Brand ${ }^{1 *}$, Elisa Wegmann ${ }^{1}$, Stephanie Antons ${ }^{1}$, Marc N. Potenza ${ }^{2}$ \\ ${ }^{1}$ University of Duisburg-Essen \\ ${ }^{2}$ Yale University, School of Medicine \\ matthias.brand@uni-due.de
}

Problematic pornography use (PPU) is a significant clinical concern for some individuals. Several authors argue that PPU has important parallels with disorders due to addictive behaviors (e.g., gambling and gaming disorders). Other authors stress parallels with impulse control disorders and subsume PPU within the broader construct of compulsive sexual behavior disorder. Irrespective of the best classification of PPU, there is debate regarding a role for moral incongruence in the context of pornography use. Moral incongruence potentially results in self-perceived pornography addiction without using pornography excessively and without fulfilling clinical criteria for a disorder due to addictive behaviors; i.e., functional 
impairments in daily life. In this contribution, recent theoretical models explaining PPU due to moral incongruence and models of addictive disorders are discussed. Although there may be some differences in terms of predisposing variables, we argue that both types of PPU share important mechanisms including craving for pornography, diminished control over use and continued use despite negative consequences. Future research and clinical practice could benefit from a more synergistic perspective rather than following two parallel lines of observations that posit orthogonality of problems related to pornography use.

\title{
SY15-5
}

\section{Ongoing debates regarding consideration and classification of compulsive sexual behavior disorder as a psychiatric diagnosis}

\author{
Marc N Potenza* \\ Yale University \\ marc.potenza@yale.edu
}

In June 2018, the World Health Organization released the 11th edition of the International Classification of Diseases and included for the first time diagnostic criteria for compulsive sexual behavior disorder (CSBD). The diagnosis was classified as an impulse control disorder, although considerable discussion and debate has occurred regarding whether CSBD should be considered as a psychiatric disorder and if so what is the most appropriate classification. This presentation will review current data on this topic, including the clinical needs of individuals presenting with problems related to CSBD. Additionally, types of sexual behaviors that may become problematic for people (e.g., pornography viewing) will also be considered with respect to CSBD. Future directions with respect to research, prevention, intervention and training will be considered.

\section{SY16-1}

\section{Pharmacological treatment of gambling disorder}

\author{
Marc N Potenza* \\ Yale School of Medicine \\ marc.potenza@yale.edu
}

Gambling disorder is experienced by about $0.5 \%$ of the general adult population. While no medications have formal indications for the treatment of gambling disorder, data from multiple randomized clinical trials suggest that certain medications may be superior to placebo in the treatment of gambling disorder. In this presentation, clinical trial data will be presented from pharmacotherapy trials. Data for opioid receptor antagonists, serotonin-reuptake inhibitors, mood stabilizers and other classes will be presented. How co-occurring symptoms and 
disorders may be informative in clinical settings will be described. The utility of combining pharmacotherapy with other empirically supported approaches (e.g., psychotherapy) will be discussed. Future directions relating to precision medicine will be considered.

\title{
SY16-2
}

\section{Mental Health and Substance Use Disorders in Gambling Disorder: What does it mean for treatment?}

\author{
David C. Hodgins ${ }^{1 *}$, Igor Yakovenko ${ }^{2}$ \\ ${ }^{1}$ University of Calgary \\ ${ }^{2}$ Dalhousie University \\ dhodgins@ucalgary.ca
}

Individuals with disordered gambling often report at least one other lifetime mental health or substance use disorder. Although prevalence estimates vary, there is substantial evidence that co-morbidity is the rule rather than the exception. This presentation will present the results of a systematic scoping review of published research that identified 251 articles. Co-morbidity is associated with more severe and persistent gambling problems, but is not necessarily linked to poorer treatment outcome. Various models explaining the association between gambling disorder and other disorders will be described. Discussion will focus on the implications of co-morbidity for how we offer treatment for gambling disorder.

\section{SY16-3}

\section{Clinical characteristics of treatment seeking individuals with Gambling Disorder in Japan: A systematic comparison of diagnostic criteria in ICD-11 and DSM-5}

\author{
Takanobu Matsuzaki*, Sachio Matsushita, Susumu Higuchi \\ Kurihama Medical and Addiction Center \\ takanobum@gmail.com
}

Background: Kurihama Medical and Addiction Center in Japan have started outpatient treatment for gambling disorder in 2013. Due to an increasing demand, we started an inpatient treatment program for gambling disorder in 2017. However, clinicaly meaningful characteristics of these Japanese patients have not been fully studied.

Objective: The aim of this study is to describe the clinical characteristics of the patients with gambling disorder in Japan. In particular, reported symptoms when using DSM-5 diagnostic criteria will be compared against ICD-11 diagnostic criteria. Methods: The participants were 113 patients who were diagnosed with gambling disorder and received CBT treatment 
at our hospital from June 2013 to April 2017. Their intake information, including demographic data and reported symptoms, were used for the analyses.

Results and discussion: Of 113 subjects, 92.0\% were male, 56.6\% were married, and $69.9 \%$ were employed. The mean age at first visit was 39.3. The mean debt at first visit was about $\$ 20,000$. The most preferred type of gambling was Pachinko / Slot machine $(90.3 \%)$ and second most preferred type of gambling was horseracing (20.4\%). By using participants' reported symptoms, ICD-11 diagnostic criteria were compared against DSM-5. The results of the analyses and clinical implications of these findings will be discussed.

\title{
SY16-4
}

\section{A novel study with naloxone nasal spray in the treatment of GD: randomized controlled study}

\author{
Hannu Alho ${ }^{1 *}$, Sari Castren ${ }^{2}$ \\ ${ }^{1}$ National Institute for Public Health and Welfare \\ ${ }^{2}$ National Institute for Health and Welfare \\ hannu.alhothl.fi
}

There is a growing interest in the use of medication assisted treatment for Gambling Disorder (GD). The strongest empirical support is for the use of opioid receptor antagonists (e.g. naltrexone and nalmefene), hypothesized to reduce opioidergic- evoked signaling within the mesolimbic and mesocortical pathways. However, oral administration of these drugs is associated with slow absorption and adverse events. Intranasal (i.n.) administration of the MOR antagonist naloxone results in a rapid increase in plasma concentrations and occupation of brain MORs.

A randomized, open label, single center study was conducted to investigate the feasibility of using an i.n. naloxone spray with brief telephone consultation in GD. Twenty participants (11 men) received "as needed" naloxone in two groups: group A (maximum dose $8 \mathrm{mg} /$ day) or in group B (maximum dose $16 \mathrm{mg} /$ day). All subjects used a mobile phone diary to record gambling, adherence to study drug and adverse events (AE). In addition they received two phone consultations during week 8 of the intervention.

Results of this study, of which the completion rate was $90 \%$, will be presented. In addition, the protocol and preliminary results of an ongoing randomized placebo-controlled study of naloxone nasal spray (maximum dose $16 \mathrm{mg}$ / day) for the treatment of GD will be presented. 


\title{
SY17-1
}

\section{Understanding the Relationship Between the Proteus Effect, Immersion, and Gender among World of Warcraft Players: An Empirical Survey Study}

\author{
Vasileios Stavropoulos*, John Rennie, Maria Morcos \\ Cairnmillar Institute \\ vas@cairnmillar.edu.au
}

Online gaming is part of contemporary life, with a range of influences on gamer 's behaviour. The convergence of an individual's behaviour with characteristics of their in-game representation (avatar), is conceptualized as the 'Proteus effect' (PE; Yee \& Bailenson, 2007). In the massively multiplayer online role-playing game (MMORPG) World of Warcraft (WoW), the Draenei ('exiled ones') are one type of in-game character. In the present study, the interplay between choosing to be a Draenei, experiencing game-related immersion, along with the gamer's biological gender was examined in relation to PE behaviours among 404 WoW gamers $($ males $=299 ; 74 \%$; females $=104 ; 26 \%)$. Participants completed the Proteus Effect Scale and the Immersion sub-scale of the User-Avatar Questionnaire (Blinka, 2008). Hierarchical regression analysis indicated a significant positive association between immersion and PE behaviours. Furthermore, mediation and moderated mediation analyses suggested that being a Draenei was positively associated with the level of game immersion, which in turn associated to higher PE behaviours offline, independent of participants' gender. Results highlight significant user-avatar bond aspects, which expand the available knowledge regarding the links between in-game and offline behaviour.

\section{SY17-2}

\section{Female gamers and their risk to develop addictive gaming behaviour}

\author{
Olatz Lopez-Fernandez* \\ Monash University \\ olatz.lopez-fernandez@monash.edu
}

Research on female gamers is scarce, but the number of women who usually play video games is increasing worldwide according to the statistical reports published this decade. Traditionally women were classed as online users who commonly used the internet with a generalised use or for social networking. However, the progressive increment of women playing 'big games' (e.g., Multiplayer Online Role-Playing Game, Multiplayer Online Battle Arena, and First-Person Shooter) are changing the figures. Thus, as the behaviour increments in this sociodemographic group, the benefits and negative consequences of video gaming are probably growing too, especially in hard-core female gamers. This presentation will show theoretical and empirical evidence on female gamers' phenomenon and their risk of devel- 
oping an addictive gaming behaviour. It will be highlighted some of their characteristics, gaming patterns, as well as their commonalities and differences compared with male gamers. In summary, it will be offered a comprehensive overview of these female gamers from an international perspective, and what it is known about their potential of developing a gaming disorder.

\title{
SY17-3
}

\section{Female patients suffering from Internet Gaming Disorder}

\author{
Lara Scherer*, Klaus Woelfling, Manfred E. Beutel, Kai W. Mueller \\ Outpatient Clinic for Behavioral Addictions Unimedizin, Mainz \\ lara.scherer@unimedizin-mainz.de
}

Introduction: Internet Gaming Disorder (IGD) represents the most common behavioral pattern of pathological internet use. Recent research reports different prevalence rates, ranging from $1 \%$ to $5 \%$. Women and men are prevalence-wise equally affected from IGD. Nonetheless, women are actually still underrepresented in the care system as well as genderspecific research.

Methods: Data were collected from a consecutive sample of 92 patients $(50 \%$ women of an Outpatient Clinic for Behavioral Addictions). The quantitative data collection assessed internet use (AICA-S; Müller et al., 2013), personality traits (NEO- FFI, Costa \& McCrae, 1992), psychological problems and symptoms of psychopathology (SCL-90R; Franke, 1995).

Results: Analysis reveals that IGD is similarly distributed in men and women. There are gender differences in time of internet use. The personality trait extraversion is significantly lower in subjects suffering from IGD. Consciousness is decreased in men and women. An increment in symptom severity has to be reported in scales for depression and paranoid ideation.

Conclusions: Former research indicates that male subjects suffer more often from IGD. Yet, women are still underrepresented in outpatient treatment. Thus, the increment in symptom severity, decreased extraversion as wells as different patterns of internet use mark an important starting point for further investigations, aiming to understand gender-specific differences. 
SY17-4

\title{
Exploring the implications of co-occurrence within Internet Addiction and Gaming Disorder
}

\author{
Tyrone Leonard Burleigh ${ }^{1 *}$, Daria J. Kuss ${ }^{2}$, Alex Sumich ${ }^{2}$, Mark Griffiths ${ }^{2}$ \\ ${ }^{1}$ Nottingham Trent University \\ ${ }^{2}$ Nottingham Trent Univeristy \\ tyrone.burleigh@ntu.ac.uk
}

Internet use and video-gaming are becoming an everyday occurrence among both the young and old. Research indicates that Internet users or gamers that experience problematic use will often experience a co-occurring behavioral or substance use related issues. Although prevalence rates may vary, it appears that the co-occurrence of behavioral addictions or substance abuse is highly likely. The current review collected data from 25 articles that considered Internet Addiction (IA), Internet Gaming Disorder (IGD), or Gaming Disorder (GD) and a co-occurring problematic behavioral or substance related use or disorder. The main findings show; (i) a scarcity of studies examining the co-occurrence of two behavioral addictions or problem behaviors; (ii) a limited amount of studies report on longitudinal risk of IA, IGD, or GD with co-occurring disorders; and (iii) there is a lack of studies examining the neurobiological mechanisms of co-occurrence in IA, IGD, or GD. The results of the present review indicate that IA, IGD, or GD co-occurs in a wide range of behavioral and substance related addictions and disorders, and that research into their co-occurrence is increasing. However, despite growing research into the area, most studies conclude high rates of co-occurrence between both behavioral and substance addictions without follow-up.

\section{SY17-5}

\section{Gaming Disorder: Evidence-based clinical practice}

\author{
Daria J Kuss ${ }^{1 *}$, Sebastiano Costa ${ }^{2}$ \\ ${ }^{1}$ Nottingham Trent University \\ ${ }^{2}$ University of Campania Luigi Vanvitelli \\ daria.kuss@ntu.ac.uk
}

Following three decades of research, in 2018, Gaming Disorder has been officially recognised as a mental disorder by the World Health Organisation, and has been included in the eleventh edition of the International Classification of Diseases (ICD-11). Gaming Disorder is being treated around the world, with approaches differing across clinics, treatment centres, and professionals providing therapy. Although the research on Gaming Disorder is growing, there is still a lack of studies assessing clinical practice. Therefore, the purpose of this talk is to present the systematically gathered evidence base on clinical practice in Gaming Disorder across the world, including the procedures used for diagnosis as well as the different types of treatments applied. Specifically, the following topics will be addressed: (i) respective socio- 
cultural research context, (ii) assessment tools, (iii) diagnostic criteria and (iv) process, and (v) the therapeutic approaches. An evaluation of the outcomes indicates that there appears to be a significant diversity in terms of the diagnostic tools, procedures, and treatment approaches used for Gaming Disorder across countries. Accordingly, researchers are advised to standardise the processes and approaches applied when working with individuals presenting with Gaming Disorder in the clinical context.

\title{
SY18-1
}

\section{School refusal and Internet addiction in adolescents with Autism Spectrum Disorder}

\author{
Kentaro Kawabe* \\ Department of Neuropsychiatry, Ehime University, Graduate School of Medicine \\ kawabe.kentaro.fj@ehime-u.ac.jp
}

The term "hikikomori" describes a Japanese psychopathological and sociological phenomenon in which people. A person of "hikikomori" is frequently occurs during not only adolescence but also adulthood, and many cases of "hikikomori" start out as school refusal. School refusal is defined as a protracted school absence caused by psychological, emotional, physical, social reasons, and other than sickness or economic causes. In addition, school refusal is frequency occurred in students with Autism spectrum disorder (ASD). Recent studies have reported that ASD children were particularly related to excessive and problematic video game and internet use. Our current study indicated that the prevalence of Internet Addiction (IA) in ASD is higher than in the general population. In addition, we also found the correlation between the high tendency of IA and the experience of school refusal in ASD adolescents. IA comorbid with ASD have the potential to occurrence of "hikikomori". It is necessary to investigate the association between school refusal and IA in detail to avoid "hikikomori" in the future among ASD adolescents. In this symposium, we will talk about the relationship between internet addiction and school refusal.

\section{SY18-2}

\section{Internet addiction among Japanese adolescents with autism spectrum disorder}

\author{
Masaru Tateno* \\ Tokiwa Child Development Center, Tokiwa Hospital / Dept. Neuropsychiatry, \\ Sapporo Medical University \\ tatema@sapmed.ac.jp
}

As the number of internet users becomes higher, problems related to internet use become more serious. Children and adolescents are susceptible to becoming addicted to the Internet, 
and those with developmental disorders are more prone to internet addiction (IA). We investigated the prevalence of IA among adolescents with autism spectrum disorder (ASD) in clinical settings. The subjects were consecutive cases with confirmed diagnosis of ASD who visited child and adolescent out-psychiatry clinic. Clinical diagnosis of IA was made by child and adolescent psychiatrists referencing diagnostic criteria for gaming disorder of ICD-11. The total of 104 subjects were involved in this study (mean age: $14.4 \pm 2.4 \mathrm{y}$, age range: 10 to $18 \mathrm{y}$, male: 80 cases). The mean Young's Internet Addiction Test (IAT) score was $43.7 \pm 12.4$ $(\mathrm{n}=104)$ and only two cases exceeded the cutoff for IA $(\geq 70)$. Based on clinical interviews by child and adolescent psychiatrists, 14 cases (13.5\%) were diagnosed as having IA with mean IAT score of $56.8 \pm 13.1$. Because individuals with ASD prone to underestimate the severity of their own problems about internet use, we often find a significant discrepancy between clinical diagnosis of IA based on face-to-face interview and the assessment about IA according to the scores on self-rating scales.

\title{
SY18-3
}

\section{Internet Addiction and Hikikomori (Oiettolie) in Korea}

\author{
Tae Young Choi ${ }^{1^{*}}$, Geun Hui Won², Dae Hun Kang ${ }^{3}$, Jin Hyeok Lee \\ ${ }^{1}$ Department of Psychiatry, Catholic University of Daegu, School of Medicine \\ ${ }^{2}$ Catholic University of Daegu, School of Medicine \\ ${ }^{3}$ Daegu Catholic University Hospital \\ tyoungchoi@cu.ac.kr
}

Hikikomori (Oiettolie) is a worldwide phenomenon and it is also found in Korea. Hikikomori seem to be associated with internet addiction. Most of those with Oiettolie had also internet addiction, which is gaining national concern. Home visit service with a structured manual turned up to have positive effect for improving their status. A step-to-step evaluation \& intervention strategy including family intervention may also help them.

There is growing interest in Internet gaming disorder (IGD), but there are no established treatment guidelines yet. Korean Adolescent with IGD has gained attention and a lot of research and projects have been done. At present, we are implementing consensual interventions rather than evidence base treatment for IGD. Therefore, not only the concept of treatment for patients but also the multifaceted intervention and management such as psychosocial approach is being studied in these days.

Many researches showed that adolescent with IGD had more problematic issues of psychopathology including depressed mood, social withdrawal, anxiety etc. Our research team has been actively studying IGD over 10 years and recently developed several psychosocial approaches and verified its effectiveness. The representative of the interventions we have developed are 'home-based daily journal' and 'group art / music therapy'. 
SY18-4

\title{
Does internet assist hikikomori-like behaviors based on the urban boundless society?
}

\author{
Takahiro A. Kato* \\ Kyushu University \\ takahiro@npsych.med.kyushu-u.ac.jp
}

Now several decades into a global Internet society and the long progression of globalization with its interflow of cultures, the time to reconsider the concept of culture-bound syndromes is upon us. Internet addiction is becoming one of the most crucial mental health issues in psychiatry especially youth. In Japan, novel socio-psychopathological phenomena such as "hikikomori", a severe form of social withdrawal, have emerged, and recent international studies have revealed that such culture-influenced phenomena have also been observed outside Japan (Kato et al. World Psychiatry 2018 Feb). A more interconnected world due to globalization and the growing importance of "(Inter)net society" may be major contributing factors behind these phenomena. An interconnected world is resulting in a boundless society and building a boundless psychological world in modern persons, which may form a variety of novel psychopathological conditions in the near future. Behind the increase of people with hikikomori there may be an interconnection with societies that are increasingly boundless. Ironically, this very interconnectedness may spur an increase in the number of people who need to drastically 'disconnect' (withdraw) from society. In this symposium, I have overviewed Japan's "hikikomori" and its related internet addiction from multi-dimensional perspectives.

\section{SY19-1}

\section{Current situation of gambling and prevalence of gambling disorders in Japan}

\author{
Toshiaki Baba ${ }^{1 *}$, Sachio Matsushita ${ }^{2}$ \\ ${ }^{1}$ National Center for Glogal Health and Medicine \\ ${ }^{2}$ National Hospital Organization, Kurihama Medical and Addiction Center \\ sachio-m@wa2.so-net.ne.jp
}

Purpose: A nationwide survey conducted in 2013 revealed a higher prevalence of gambling disorder compared with other countries based on results of the South Oaks Gambling Screen (SOGS) in Japan. The present study aimed to investigate gambling behaviors and confirm the results of that survey.

Subjects and Methods: We investigated general gambling behaviors among 10,000 randomly-selected Japanese participants aged 20-74 years using semi-structured interviews. The participants also completed the SOGS. The Ethics Committee at Kurihama Medical and Addiction Center approved the study protocol. 
Results: The overall response rate was $53.7 \%$. The results indicated that $80.6 \%$ of men and $57.2 \%$ of women had gambled during their lifetime and $41.9 \%$ and $24.4 \%$ respectively, had gambled during the previous year. The lifetime SOGS score was $\geq 5$ (95\% CI: $3.1-4.2 \%$ ) for $3.6 \%$ of the respondents, compared with $0.8 \%$ during the previous 12 months $(95 \% \mathrm{CI}$ : $0.5-1.1 \%)$.

Conclusions: The prevalence of lifetime SOGS scores $\geq 5$ was higher in Japan than in other countries, suggesting that periodic gambling surveys are needed to understand the actual situation of gambling disorder in Japan.

\title{
SY19-2
}

\section{Gambling Disorder in Korea - The Status and Management of Gambling Disorder Based on Community}

\author{
Aeran Park*
}

General Manager, Department of Prevention and Public Relations of Korea Center on Gambling Problems aenapark22@kcgp.or.kr

Korea Center on Gambling Problems (KCGP) provides information, support and counselling for the prevention and treatment of problem gambling. Especially individual and group counselling services apply cognitive behavioral therapy, motivational interviewing and 12 steps. KCGP operates the National Gambling Helpline (nation-wide number 1336) and 14 regional centers, provides treatments for problem gamblers and their families in collaboration with local counselling agencies and hospitals. Helpline services is free, available 24 hours, seven days a week. Helpline counsellors provide confidential advice, information and emotional support to anyone experiencing problems with gambling, and connect people with regional centers or local agencies for regular and more convenient services.

KCGP is here to help people affected by gambling harm, and to prevent and minimize the risk and potential harm from gambling in society. KCGP intends to become the "light of hope' for gamblers suffering from the 'night of gambling', and a competent guide for participants of gambling not to be problem gamblers.

The outcomes of KCGP's prevention and treatment project are institutional and 1336 recognition, gambling problem awareness, gambling disorder prevalence, quality of life, gambling abstinence, frequency of gambling, Improvement of family life, occupational life, leisure life and self-help group participation. 
SY19-3

\section{Development and efficacy evaluation of an Internet-delivered intervention integrated into a popular messaging app for gambling problems}

Ryuhei So*

Okayama Psychiatric Medical Center

nexttext@gmail.com

Nine out of ten problem gamblers are reluctant to take face-to-face treatments. To reduce the considerable treatment gap, we tried to develop an Internet-delivered intervention named GAMBOT which was integrated into a popular messaging app for problem gamblers. Also, we also conducted a randomized controlled trial (RCT) to investigate the efficacy of GAMBOT in 2018. In my presentation, I will overview the current progress of Internet-delivered interventions for problem gamblers. Then I will introduce the contents of GAMBOT and the results of the RCT such as the characteristics of the participants, the usage data, and the efficacy outcomes.

SY19-4

\section{Dissemination and Implementation of Cognitive Behavioral Therapy for Gambling Disorder in Public Health and Social Service Settings in Japan}

\section{Keiji Kobara*, Hiroshi Sato}

Shimane Prefectural Counseling Center for Physical and Mental Health keijikobara@gmail.com

Few medical institutions can apply cognitive behavioral therapy (CBT) for gambling disorder (GD) in Japan, and opportunities are limited further in the fields of public health and social services. Therefore, we developed 2 programs suitable for dissemination and implementation of CBT programs for GD in these fields. The first is Shimane Addiction Recovery Training Program for Gambling Disorder (SAT-G), which comprises a 5-session CBT program. Each session lasts approximately 1.5 hour. The second is SAT-G-Light, the simplified version of SAT-G. It comprises a 3-session CBT program. Each session lasts about one hour. We have also found that SAT-G-Light can be safely applied to dual-diagnosed GD patients, with schizophrenia or mental retardation, if the support staff, e.g. welfare case workers, can fully attend the SAT-G Light sessions. The details of two programs will be given at the symposium. 


\title{
SY20-2
}

\section{Relation between erectile dysfunctions and pornography use - analysis of large set of self-reported data}

\author{
Ewelina Kowalewska ${ }^{*}$, Urszula Hamerska ${ }^{2}$, Marc N. Potenza ${ }^{3}$, Mateusz Gola ${ }^{4}$ \\ ${ }^{1}$ SWPS University of Social Sciences and Humanities, Department of Psychology, Warsaw, PL \\ ${ }^{2}$ SWPS University of Social Sciences and Humanities, Department of Psychology, Wroclaw, PL \\ ${ }^{3}$ Yale University School of Medicine, Department and Neuroscience and Child Study Center, \\ New Haven CT, USA \\ ${ }^{4}$ Swartz Center for Computational Neuroscience, Institute for Neural Computations, \\ University of California San Diego, CA, USA \\ ekowalewska@swps.edu.pl
}

Aims: Development of technology has made pornography easily accessible. As a result of an increase consumption of pornography videos, more and more of its users complaining about difficulties in mental, sexual and social functioning. Methods: Analysis covered 230 self-reports of males (aged 18-22) describing improvements in sexual and psychosocial functioning after abstaining from pornography consumption.

Results: Among all analyzed sexual dysfunctions the most noticeable problem was Erectile Dysfunction during sexual activity. $83 \%$ males reported difficulties in sustaining erection before quitting porn, $5 \%$ - during the process of quitting porn, and only $1 \%$ - after quitting porn. Furthermore, $6 \%$ of males reported severe decline in [or loss of] libido before quitting pornography, $7 \%$ had low sexual desire during quitting pornography, and 7\% reported improvement in this aspect after giving up pornography use. In case of withdrawal symptoms, the most frequently reported was depression $(33 \%)$, emotional numbness $(33 \%)$, social anxiety $(22 \%)$. However, according to testimonies in $27 \%$ of cases there was an improvement of social and emotional functioning due to the pornography quitting.

Conclusions: These findings provide evidence of negative impact of pornography consumption on social and emotional aspects of life, and improvement of functioning in these areas after quitting pornography. 
SY20-3

\title{
Pornography use, Self-Reported Addiction, and Erectile Functioning: Results from Nationally Representative and Longitudinal Data-Sets
}

\author{
Joshua B. Grubbs ${ }^{1 *}$, Mateusz Gola ${ }^{2}$ \\ ${ }^{1}$ Bowling Green State University \\ ${ }^{2}$ Clinical Neuroscience Laboratory, Institute of Psychology, Polish Academy of Sciences \\ GrubbsJ@BGSU.edu
}

Many popular advocacy groups have advanced the claim that pornography use is driving a burgeoning epidemic of erectile dysfunction in otherwise healthy men. Even so, little prior empirical work has attempted to test these claims. As such, the present work used three samples - an online sample of men matched to U.S. nationally representative norms, a sample of undergraduate men at a university in the U.S., and a 12-month longitudinal convenience sample of adult men in the U.S. - to examine whether or not pornography use, self-reported pornography related problems, and erectile dysfunction were related. Results generally indicated that mere pornography use itself was most often unrelated to erectile functioning, but self- reported problems with pornography use were both cross-sectionally and longitudinally associated with diminished erectile functioning. However, erectile functioning demonstrated no change over time. Collectively, these results suggest that self- reported problems with pornography use (i.e., feelings of addiction) are likely associated with erectile functioning, but these links are not directional or causal in nature.

\section{SY20-4}

\section{Facets of Personality in Hypersexual Patients with Adult ADHD}

\author{
Rory C. Reid*, Margarit Davtian, Timothy W. Fong \\ University of California, Los Angeles \\ roryreid@gmail.com
}

\begin{abstract}
Background and Aims: A significant number of individuals seeking help for hypersexual behavior also meet diagnostic criteria for adult ADHD with predominantly inattentive symptoms. In order to elucidate unique characteristics in this sub-population of hypersexual men, the current study explored facets of personality in a treatment seeking sample of hypersexual patients with $(\mathrm{n}=110)$ and without $(\mathrm{n}=108)$ adult ADHD using the NEO Personality Inventory - Revised. Methods: Participants completed study measures and were recruited from outpatient clinics in the USA upon informed consent. Results: Findings suggest that both groups experience emotional instability, impulsivity, stress-proneness, difficulties with self-regulation, motivation, and casual attitudes toward adherence to moral or ethical principles. Interestingly, hypersexual patients with and without ADHD reported comparable levels of hypersexual behavior and impulsivity. Discussion and Conclusions: Several differences between the groups emerged across facets of personality suggesting hypersexual patients with ADHD may encounter some additional challenges beyond those faced by non-ADHD hypersexual patients.
\end{abstract}




\title{
SY20-5
}

\section{Brief Pornography Screener: Identifying problematic pornography use in a U.S. sample}

\author{
Shane W Kraus ${ }^{1 *}$, Joshua B Grubbs ${ }^{2}$ \\ ${ }^{1}$ VISN 1 New England MIRECC, Edith Nourse Rogers Memorial Veterans Hospital, MA USA \\ ${ }^{2}$ Bowling Green State University \\ swkraus@gmail.com
}

Background and aims: The Brief Pornography Screener is a five-item self-report questionnaire developed to detect problematic use of pornography among clinical and non-clinical samples. Preliminary data suggests that U.S. veterans experience higher rates of problematic pornography use. Methods: We recruited a U.S. nationally representative cross-sectional sample of 1461 adult pornography users to assess problematic use of pornography and compared veterans vs. non-veterans on BPS scores and other pornography use-related characteristics. Results: Of the 1461 adult pornography users surveyed, $17.6 \%(\mathrm{n}=258)$ were military veterans. Veterans reported higher levels of problematic pornography use $(\mathrm{M}=2.61, \mathrm{SD}=$ $3.42)$ as compared to non-veterans $(\mathrm{M}=1.34, \mathrm{SD}=2.23)$. Regression analysis also revealed that isolated pornography viewing $(\mathrm{t}=13.86, \mathrm{p}<0.001)$, time spent watching pornography $(\mathrm{t}=3.76, \mathrm{p}<0.001)$, and veteran status $(\mathrm{t}=7.12, \mathrm{p}<0.001)$ were significant predictors of problematic pornography use. Conclusions: We found that solitary and frequent pornography viewing, as well as being a U.S. Veteran, were strongly associated with problematic use of pornography. Our findings suggested additional screening for problematic pornography use and other compulsive sexual behaviors among veterans is warranted to ensure that these individuals receive appropriate mental health care services.

\section{SY21-1}

\section{Internet game advertisements and youth internet game addiction: Revisiting social responsibility}

\author{
Sulki Chung ${ }^{1 *}$, Jaekyoung Lee ${ }^{2}$, SooBi Lee ${ }^{1}$, Suyoung Lee ${ }^{1}$, Jiseon Kim ${ }^{1}$ \\ ${ }^{1}$ Chung-Ang University \\ ${ }^{2}$ Korea Center on Gambling Problems \\ chungs@cau.ac.kr
}

Objectives: To date, socio-environmental influence relating to internet game addiction has received less attention. In order to raise awareness of social responsibility in preventing internet game addiction, this study examined the extent of exposure to internet game advertisements and the risk of internet game addiction among youths. 
Methods: The sample consisted of 1,838 middle school students living in Seoul and Gyeonggi province in Korea, who completed a survey measuring exposure to internet game advertisements and other game related behaviors.

Results: About $6 \%$ of the respondents were classified as the internet game addiction risk group. Respondents were exposed to internet game advertisements through various media including TV, application search windows and ads posted in public transportation. The risk group showed higher perception of game advertisements. Multiple logistic regression results indicated that the exposure to advertisements and receptivity of the advertisements increased the risk of internet game addiction. Conclusions: Study findings confirmed the role of environmental factors associated with internet game addiction. In order to decrease and prevent internet game addiction, more attention should be given to strategies targeting social responsibility of the game industry and the society. More effective regulation and self-regulation efforts in regards to game advertisements are needed.

\title{
SY21-2
}

\section{A psychosocial developmental model for understanding adolescence gambling problems in Korean society}

\author{
Yena Kim ${ }^{1 *}$, Sook-Hee $\mathrm{Im}^{2}$, Sun Jung Kwon ${ }^{1}$, Esther Kim ${ }^{1}$ \\ ${ }^{1}$ Korea Baptist Theological University \\ ${ }^{2}$ Konyang Cyber University \\ suki0070@kycu.ac.kr
}

The problem of adolescence gambling in Korean society has reached a serious level, which is spreading very quickly through mobile phone (smart phone). Thus, it is necessary to carry out systematic and integrated longitudinal study to explore the process of adolescence gambling on the following topics:

(1) What are the initial developmental factors (opportunities, experiences and initial conditions) of the risk gambling behavior and gambling problems of adolescents? (e.g., such as buying loot boxes in game)?

(2) What are the controlling biopsychosocial risk factors involved in the development of gambling problems?

(3) What are the biopsychosocial protection factors that contribute to the natural recovery of problem gambling even under risk conditions?

To this end, we summarized the relevant variables of adolescence gambling found in the previous study and conducted qualitative studies based on ground theory for adolescents who met the conditions. By synthesizing these results, we have derived a psychosocial developmental model specialized in Korea. In order to verify the model, we constructed the measurement items and collected data covering 4,000 adolescence online panels twice a year (every 6 months). Based on the data, this study examined temporal changes in the gambling addiction of adolescents and related psychological variables using a prospective design. We also discussed what suggests prevention and treatment of adolescence gambling addiction based on the results. 
SY21-3

\title{
A cross-cultural study on the mediating effect of perceived social support between stress / negative emotions and problematic smartphone use in Chinese and Belgian undergraduates
}

\author{
Tieqiao Liu ${ }^{1 *}$, Jiang Long ${ }^{1}$, Yueheng Liu ${ }^{1}$, Aurore Pottie ${ }^{2}$, Yingying Wang ${ }^{l}$, Qiuxia Wu ${ }^{1}$, \\ Yuejiao Ma ${ }^{1}$, Joel Billieux ${ }^{3}$ \\ ${ }^{1}$ Mental Health Institute of the Second Xiangya Hospital, Central South University \\ ${ }^{2}$ Laboratory for Experimental Psychopathology, Psychological Science Research Institute, \\ Universite Catholique de Louvain \\ ${ }^{3}$ Addictive and Compulsive Behaviours Lab (ACB-lab), Institute for Health and Behaviour, \\ University of Luxembourg \\ long-jiang@foxmail.com
}

Background: Since problematic smartphone use (PSU) is culture-dependent, the study aims to identify cultural differences regarding the potential mediating effect of perceived social support in the east and west backgrounds.

Methods: A stratified convenience sampling strategy was performed in China and Belgium. 2380 Chinese and 1001 Belgian undergraduates completed an online survey. The survey consisted of socio-demographics, smartphone use features, Problematic Mobile Phone Use Questionnaire, Multidimensional Scale of Perceived Social Support, and Depression Anxiety Stress Scale. Structural Equation Modeling analysis was computed to explore the mediating effect of perceived social support between anxiety / depression / stress and PSU.

Results: Comparing with their Belgian peers, Chinese undergraduates tended to spend significantly more time on smartphone use and prefer recreational functions of smartphones (e.g. mobile gaming, video watching). Perceived family support exerts partial mediating effect in the "anxiety $\rightarrow$ PSU" path, and the mediating effect exists in both cultural backgrounds. Perceived family support exerts partial mediating effect in the "stress $\rightarrow$ PSU" path among Chinese undergraduates, however, such mediating effect was not detected among their Belgian peers.

Conclusions: Cultural differences between Chinese and Belgian undergraduates exist in smartphone use patterns, as well as with regard to the mediating effect of perceived social support between stress and PSU. 
SY21-4

\title{
Individual characteristics and coping mechanisms associated with the self-stigma of problem gambling
}

\author{
Nerilee Hing*, Alex Myles, Thomas Russell \\ Central Queensland University \\ n.hing@cqu.edu.au
}

Few studies have examined the stigma of problem gambling and internalisation of this prejudice as damaging self-stigma. This study aimed to identify psychological factors, sociodemographic characteristics and coping mechanisms associated with the self-stigma of problem gambling. An online survey was conducted of 177 Australian adults with a current gambling problem to measure self-stigma, self-esteem, social anxiety, self-consciousness, psychological distress, symptom severity, most problematic gambling form, stigma coping mechanisms and sociodemographic characteristics. A multivariate linear regression indicated that higher levels of self-stigma were associated with: being female, being older, lower self-esteem, higher problem gambling severity score, and greater use of secrecy. Strongest predictors were self-esteem and symptom severity score. These results suggest that the self-stigma of problem gambling may be driven by similar mechanisms as the self-stigma of other mental health disorders, and impact similarly on self-esteem and coping. Thus, self-stigma reduction initiatives used for other mental health conditions may be effective for problem gambling. The self-stigma of problem gambling increased with female gender and older age, which are associated with gaming machine problems. This group should be a focus for efforts to reduce or better cope with the self-stigma of problem gambling.

SY22-1

Internet-communication disorder in German children and adolescents The interaction of age, need to belong, and self-regulation

\author{
Sina Ostendorf ${ }^{1^{*}}$, Benjamin Stodt ${ }^{1}$, Matthias Brand ${ }^{2}$ \\ ${ }^{1}$ General Psychology: Cognition and Center for Behavioral Addiction Research (CeBAR), University \\ of Duisburg-Essen, Duisburg, Germany \\ ${ }^{2}$ General Psychology: Cognition and Center for Behavioral Addiction Research (CeBAR), University \\ of Duisburg-Essen, Duisburg, Germany; Erwin L. Hahn Institute for Magnetic Resonance Imaging, \\ Essen, Germany \\ sina.ostendorf@uni-due.de
}

Background: Since many people interact via online-communication applications, there is a remarkable number of especially younger people using such applications excessively despite negative consequences. This is also termed Internet-communication disorder (ICD). Based on recent theoretical models, this study focuses on the interaction of personal attri- 
butes (need to belong, NTB, and age) and specific competences (online self-regulative competences, OSRC) in the prediction of ICD. Methods: Children and adolescents $(\mathrm{N}=475$, 10-17 years) answered different questionnaires covering ICD-symptoms, OSRC, and NTB. Results: Moderated regression analysis revealed significant main effects for NTB, age, and OSRC in the prediction of ICD-symptoms and a significant three-way interaction. Individuals with high NTB and low OSRC showed highest ICD- symptoms, especially when being older. With increasing OSRC, ICD-tendencies significantly decreased for younger and older individuals. However, young individuals with high NTB and high OSRC showed less ICDsymptoms than older ones. Older individuals even had high ICD-symptoms when NTB was low, if OSRC were low as well. Conclusions: OSRC can reduce ICD-symptoms in especially younger children having high NTB. But even if NTB is low, particularly older individuals need good OSRC to reduce ICD-tendencies. This strengthens the importance of improving protective competences to prevent from an overuse of online-communication applications.

\title{
SY22-2
}

\section{Is Internet gaming disorder associated with general or gaming-specific neural alterations in emotional processing?}

\author{
Benjamin Becker ${ }^{1 *}$, Fangwen Yu ${ }^{1}$, Christian Montag ${ }^{2}$, Bernd Lachmann ${ }^{2}$, Rayna Lachmann ${ }^{2}$, \\ Feng Zhou ${ }^{1}$ \\ ${ }^{1}$ NeuSCAN Lab, The Clinical Hospital of Chengdu Brain Science Institute, MOE Key Laboratory for \\ Neuroinformation, University of Electronic Science and Technology of China, Chengdu, China \\ ${ }^{2}$ Institute of Psychology and Education, Ulm University, Ulm, Germany \\ yufangwen@yeah.net
}

Introduction: Accumulating evidence suggests that substance use disorders are characterized by altered neural processing of drug-associated stimuli (cue-reactivity) as well as general emotion processing impairments. Despite increasing evidence for exaggerated neural reactivity to gaming-related cues in excessive Internet gamers alterations in general emotional processing have not been examined.

Methods: $\mathrm{N}=36$ excessive Internet gamers (World of Warcraft, WoW) and $\mathrm{n}=34$ carefully matched gaming naïve controls underwent a block-design functional MRI paradigm including gaming-associated (WoW) stimuli, gaming-unrelated positive and negative emotional stimuli as well as neutral control stimuli.

Results: In line with previous studies excessive gamers exhibited exaggerated neural reactivity to gaming-related cues, specifically in the posterior cingulate cortex (whole-brain analyses, FDR corrected $\mathrm{p}<.05$ ). In contrast, excessive gamers did not demonstrate altered neural processing of gaming-unrelated emotional stimuli.

Conclusions: Findings suggest gaming cue-related exaggerated reactivity in regions engaged in social and self-referential processing in excessive gamers. However, in contrast to subjects with substance use disorders, excessive gamers demonstrated intact emotional processing. 
SY22-3

\title{
Higher number of screen unlocks is positively linked to smartphone-use disorder: A real-world tracking approach
}

\author{
Christian Montag ${ }^{1 *}$, Harald Baumeister ${ }^{2}$, Christopher Kannen, Rayna Sariyska ${ }^{2}$, \\ Eva-Maria Rathner', Matthias Brand ${ }^{3}$ \\ ${ }^{1}$ Ulm University and University of Electronic Science and Technology of China \\ ${ }^{2}$ Ulm University \\ ${ }^{3}$ University of Duisburg-Essen \\ christian.montag@uni-ulm.de
}

Background: Recent research provided first evidence that high frequent smartphone use might result in less productive behavior at work. Furthermore, such a link could be explained by a high number of daily interruptions. Given problems in assessing one's own actual smartphone use via self-report, we aimed to directly record how often people unlock their phones and how this is linked to self-reported smartphone-use disorder (SUD).

Method: Smartphone usage of $\mathrm{N}=108$ participants was tracked via the application "Insights" for 12 days to get insights into their smartphone usage. Moreover, all participants filled in the smartphone-addiction-scale (SAS) by Kwon et al. (2013).

Results: $\mathrm{M}=49.05$ ( $\mathrm{SD}=26.88$ ) daily 'screen unlocks' could be observed in the present sample. Moreover, a positive association between number of 'screen unlocks' and selfreported SUD tendencies could be established $(\mathrm{rho}=.36)$. The association between 'screen unlocks' and the SAS' subscale called cyberspace-oriented relationship was in particular strong (rho $=.42$ ) underlining the idea that smartphones are strongly used to communicate via social media applications. Hence mobile Internet- communication disorder might explain large parts of SUD.

Discussions: The present work demonstrates both the feasibility and importance to include actual recorded real-world data to better understand technology-related-use disorders.

\section{SY22-4}

\section{The potential of a sports intervention as a therapy for Internet-use disorder and Internet-gaming disorder: Preliminary Results}

\author{
Rayna Sariyska ${ }^{1 *}$, Christian Montag ${ }^{2}$ \\ ${ }^{1}$ Institute of Psychology and Education, Ulm University, Ulm, Germany \\ ${ }^{2}$ Institute of Psychology and Education, Ulm University, Ulm, Germany; The Clinical Hospital of \\ Chengdu Brain Science Institute, MOE Key Lab for Neuroinformation, University of Electronic \\ Science and Technology of China, Chengdu, China \\ rayna.sariyska@uni-ulm.de
}

More and more studies are conducted to investigate the phenomena of Internet-use disorder (IUD) and Internet-gaming disorder (IGD). The latter was recognized as an official disorder in ICD-11 (Gaming disorder, predominantly online), leading to the proposal of a set of cri- 
teria to diagnose IGD. This development enables the more systematic research on different intervention approaches and their efficacy in the treatment of IUD and IGD.

The current research project aimed at examining the effect of a sports intervention (here running) on the symptoms of IUD and IGD. N (target sample size) $=75$ were tested in the present study. Next to postulated reductions in IUD and IGD symptoms, the effect of the sports intervention on cognitive variables and life satisfaction to name a few was examined. For the purpose of the study, three groups of participants were compared: EG1 and EG2 included participants with problematic tendencies towards IUD / IGD, whereas the CG demonstrated "healthy" Internet use. Moreover, only EG1 participated in the sports intervention, which included 10 minutes of warm up and stretching exercises plus 20 minutes of running on three days of the week for four consecutive weeks. The physical activity of participants was tracked using a smartphone application. Preliminary results will be presented and the potential of sports therapy for reducing IUD and IGD symptoms will be discussed.

\title{
SY23-1
}

\section{The estimated prevalence of ICD-11 gaming disorder among representative young people in the general population in Japan}

\author{
Susumu Higuchi ${ }^{1 *}$,Hideki Nakayama ${ }^{1}$, Satoko Mihara ${ }^{1}$, Aya Kinjo ${ }^{2}$, Yoneatsu Osaki², Hans \\ Jurgen Rumpf ${ }^{3}$
}

${ }^{1}$ National Hospital Organization Kurihama Medical and Addiction Center, Japan

${ }^{2}$ Tottori University School of Medicine, Yonago, Japan

${ }^{3}$ University of Lubeck, Germany

h-susumu@db3.so-net.ne.jp

Introduction: Gaming disorder (GD) has become a worldwide concern in relation to health, social and familial functioning. However, the actual conditions relating to ICD-11 GD are poorly understood, due to the need for further development of instruments to accurately evaluate gaming disorder. For this study we conducted a survey to estimate the prevalence of GD and related conditions, including DSM-5 Internet gaming disorder, among young people in the general Japanese population.

Methods: This study consists of two surveys with identical methodology. The participants of the first study were 6,000 Japanese males and females, aged between 10 and 29 years old. They were identified via a two-step stratified random sampling method. A 12-page, selfreported questionnaire, including both newly developed and existing components to operationalize the definition of GD, was administered in February, 2019. The second survey was conducted among a further 3,000 participants in March 2019.

Results: The response rate of the first survey was approximately $58 \%$. We will show the results of these surveys at ICBA2019. 
SY23-2

\title{
World Health Organization (WHO) Collaborative Project on the Development of an International Diagnostic Interview for Gaming Disorder
}

\author{
Natacha Carragher ${ }^{*}$, Hans-Jurgen Rumpf ${ }^{2}$, Susumu Higuchi ${ }^{3}$, Joel Billieux ${ }^{4}$, Daniel King ${ }^{5}$, \\ Henrietta Bowden-Jones ${ }^{6}$, Sophia Achab ${ }^{7}$, Max Abbott ${ }^{8}$, Afarin Rahimi-Movaghar ${ }^{9}$, \\ Hao Wei ${ }^{10}$, Osman Tolga Aricak ${ }^{11}$, Claudia Gandin ${ }^{12}$, Emanuele Scafato ${ }^{12}$, David Hodgins ${ }^{13}$, \\ Michael Biagent ${ }^{14}$, Zsolt Demetrovics ${ }^{15}$, Thomas F. Babor ${ }^{16}$, John Saunders ${ }^{17}$, \\ Vladimir Poznyak ${ }^{1}$ \\ ${ }^{1}$ World Health Organization \\ ${ }^{2}$ University of Lubeck \\ ${ }^{3}$ Kurihama Medical and Addicition Centre \\ ${ }^{4}$ University of Luxembourg \\ ${ }^{5}$ University of Adelaide \\ ${ }^{6}$ Imperial College \& National Problem Gambling Clinic \\ ${ }^{7}$ University Hopsital of Geneva \\ ${ }^{8}$ Auckland University of Technology \\ ${ }^{9}$ Tehran University of Medical Sciences \\ ${ }^{10}$ Central South University \\ ${ }^{11}$ Hasan Kalyoncu University \\ ${ }^{12}$ National Institute of Health Italy \\ ${ }^{13}$ Unviersity of Calgary \\ ${ }^{14}$ Drug and Alcohol Services South Australia \\ ${ }^{15}$ Eotvos Lorand University \\ ${ }^{16}$ University of Connecticut \\ ${ }^{17}$ University of Queensland carraghnat@who.int
}

Video-gaming is a popular activity and healthy hobby for the majority of players. However, in recent years, there has been increasing global recognition that excessive online video gaming may lead to marked functional impairment and psychological distress for a significant minority of players. In an increasing number of countries and jurisdictions, the problem has reached the magnitude of a significant public health concern. In response, in 2013, internet gaming disorder was included in the DSM-5 appendix section for conditions requiring further study. In 2018, gaming disorder was included in ICD-11. These changes in classification underscore the need for a psychometrically robust assessment instrument, however, no gold standard currently exist. Several research groups have identified inconsistencies in current gaming instruments and called for a consensus on measurement to ensure consistent assessment. This presentation outlines a project currently underway under the auspices of the World Health Organization to develop a new diagnostic interview for gaming disorder. The diagnostic interview is being developed as part of an international, multi-site collaboration. It involves consumer input, expert consultation (clinicians, public health professionals, researchers) and psychometrics. The interview will yield diagnoses, enable identification of comparable prevalence rates across countries and provide important scaffolding for treatment decisions. 
SY23-3

\title{
Concordance of the ICD-11 and DSM-5 classification approaches on grounds of standardized interview data
}

\author{
Hans-Jürgen Rumpf ${ }^{{ }^{*}}$, Dominique Brandt ${ }^{1}$, Jennifer Winter ${ }^{l}$, Bettina Besser ${ }^{l}$, \\ Anja Bischof ${ }^{1}$, Christian Meyer ${ }^{2}$,Ulrich John ${ }^{2}$, Gallus Bischof ${ }^{1}$ \\ ${ }^{1}$ University of Lübeck, Department of Psychiatry and Psychotherapy \\ ${ }^{2}$ University Medicine Greifswald, Institute of Social Medicine and Prevention \\ hans-juergen.rumpf@uksh.de
}

Background and aims: Besides the classification of Internet Gaming Disorder introduced as a condition for further study in DSM-5, ICD-11 will include Gaming Disorder as a behavioural addiction. Both approaches use different sets of criteria. The aim of this paper is to look at the convergence of both approaches. Methods: Findings are based on three samples: a general population-based subsample and samples recruited in unemployment centres and vocational schools. After initial screening, participants fulfilling 21 or more points on the Compulsive Internet Use Scale (CIUS) received a fully standardized diagnostic interview based on the principles of the Composite International Diagnostic Interview (CIDI). Results: When focusing on the subgroup of gamers, prevalence rates in this screening positive subgroup were the same (11.9\%) according to DSM-5 and ICD-11. Concordance between both diagnoses was as indicated by a kappa of 0.60 , however concordance differed between samples. Endorsement rates for the DSM-5 approach showed marked differences with the lowest in "jeopardizing". The three ICD-11 criteria were more similar and ranged between $27 \%$ and $48 \%$. Conclusions: This is one of the first studies on the concordance of DSM-5 and ICD-11 according to (Internet) Gaming Disorder. Findings suggest that both approaches lead to similar results.

\section{SY23-4}

\section{Concordance of gaming disorder in ICD-11 and DSM-5 based on a statewide representative survey of ninth-graders}

\author{
Florian Rehbein* \\ Criminological Research Institute of Lower Saxony, Hanover, Germany \\ florian.rehbein@kfn.de
}

In 2013, Internet gaming disorder (IGD) was included in Section 3 of the Diagnostic and Statistical Manual of Mental Disorders (DSM-5) as a condition for further study. A threshold of five out of nine proposed criteria was recommended for diagnosis (polythetic classification). In the 11th revision of the International Classification of Diseases (ICD-11), gaming disorder (GD) is supposed to be included, comprising five criteria out of which all are recommended for diagnosis (monothetic classification). However, empirical data on how these two approaches compare to each other are still scarce. In the years of 2013, 2015, and 2017, 
a statewide representative survey of ninth-graders was conducted in Lower Saxony (Germany). The pooled dataset comprises about $\mathrm{N}=29.000$ students. IGD and GD were assessed with the Video Game Dependency Scale (CSAS) that covered all IGD and GD criteria. In the talk, the following questions will be addressed: 1 . How do the 12-month prevalence rates of IGD respectively GD in German Ninth-Graders compare using the classifications of the DSM-5 and ICD-11? 2. How many respondents are classified exclusively according to DSM-5, exclusively according to the ICD-11 and concordantly within both classification approaches? 3. How do classified students out of different classification groups compare regarding impairment?

\section{SY24-1}

\section{Considering the countermeasure against gambling disorder from imaging studies}

\section{Kosuke Tsurumi*}

Kyoto University Graduate, School of Medicine

kaytee@kuhp.kyoto-u.ac.jp

Gambling disorder (GD) is a chronic mental disorder which affects the well-being of patients as well as that of people around them by huge debts and / or interpersonal problems caused by patients' excessive gambling behaviors. Although imminent casino opening and quite high prevalence of GD in Japan compared to that in other countries, countermeasures to tackle GD is insufficient. This might be partly because of the lack of effective pharmacotherapy leading to large treatment gap and low treatment persistence rate. Investigating the neural basis of GD could contribute to revealing the clinical marker which could give a clue to better intervention and / or prevention. With the aim of elucidating neural basis of GD, we are conducting imaging studies. Some of them revealed the association between neural activity and duration of illness. This implies the importance of early diagnosis and intervention. Others of them revealed the heterogeneity of GD. This might imply the importance of subtypebased intervention and / or prevention. These results would give some help to consider the countermeasures of GD. 
SY24-3

\title{
The process of gambling problem getting serious
}

\author{
Kiyomi Arai* \\ Tokyo Metropolitan University \\ k-arai@tmu.ac.jp
}

Gambling disorders in Japan mainly involve pachinko and pachi-slot. While these games can be easily enjoyed, they also pose a danger in that they are highly addictive, and when people fall into debt, their gambling addiction may not readily become apparent. This is a major factor that makes it difficult to carry out interventions for gambling disorder. There are six stages in the escalation process of a gambling disorder. People progress to gambling disorders while repeatedly advancing and recovering along these stages. In stages 1 and 2, results that can satisfy the motivation to gamble are obtained. Up to stage 4, the motivation to gamble is fulfilled, but it becomes problematic. Undesirable results are obtained in stages 5 and 6 , which can be viewed as the stages in which consciousness is focused on money. Among these six stages, stages 1-4 are those in which results may be obtained that are in line with expectations, and in stages 5-6, the gambler starts to obtain undesired results. In the present study, the factors leading to an aggravation of gambling problems as well as the psychological status of the persons involved are considered, as are approaches that take these into account.

\section{SY24-4}

\section{Early detection of gambling disorder based on gambling behavioral data}

Isamu Watanabe ${ }^{1 *}$, Hiroko Suzuki ${ }^{1}$, Gen Shinozaki ${ }^{2}$, Tomohiro Takagi ${ }^{3}$

${ }^{1}$ Fujitsu Laboratories Ltd

${ }^{2}$ University of Iowa

${ }^{3}$ Meiji University hiroko.suzuki@fujitsu.com

In order to prevent gambling disorder, it is important to detect early signs and symptoms of problem gambling behavior before the situation worsens. In recent years, with the spread of online gambling and Internet games, prediction and early detection of gambling disorder based on online behavioral data is becoming possible. The purpose of this research is to build such a predictive model and to clarify risk factors leading to problem gambling behavior.

In this research, we used data such as online gambling dataset provided by Harvard Medical School $(\mathrm{N}=4,134)$. Responsible gambling events in the dataset, such as account closure due to problem gambling, were used for the targets for prediction. Frequency-based statistics, such as the number of bets per day, and monetary-based statistics, such as the amount of money per bet, were calculated from personal gambling record as basic predictors of the 
model. In addition to the average value, the variability and the temporal change of the basic predictors were also used as predictors. With the model, it was possible to predict problem gambling behavior with high accuracy and some strong risk factors, such as types of games played, were found.

\title{
SY25-1
}

\section{Does mate preference predict problematic online pornography? Cross-cultural findings.}

\author{
Lijun Chen ${ }^{1 *}$, Zsolt Demetrovics ${ }^{2}$, Marc N. Potenza ${ }^{3}$ \\ ${ }^{1}$ School of Humanities and Social Sciences, Fuzhou University, China \\ ${ }^{2}$ Institute of Psychology, Eotvos Lorand University, Budapest, Hungary \\ ${ }^{3}$ Department of Psychiatry, Yale University, School of Medicine, USA \\ qqkx159@126.com
}

A total of 429 Chinese were tested and 395 of Hungarian were evaluated by scale method. In this study, four questionnaires were included in the questionnaire design, namely, Mate Preference Scale, Relationship Assessment Scale, UCLA Loneliness Scale, Problematic Pornography Consumption Scale.We calculated the type of preference preference, tested the chain medium model, and compared the cross - cultural consistency of the chain medium model. Results: There are significant differences in mate preference between the Chinese and Hungarian subjects. Chinese subjects pay more attention to the social status of the partner and the temperate, intellectual and dominant qualities, while the Hungarian subjects focus on passion and attach importance to their partners' sexual attraction. Construct chain intermediary model, its fitting index CFI $=0.923$, TLI $=0.910$, RMSEA $=0.61$, as acceptable model. Through bootstrap test multiple intermediary effects, the result show that mate preference predict pornography use through relationship satisfaction and loneliness, the mediating effect was -0.04 . Multi-group test found that the chain intermediary model did not have crosscultural consistency. The relationship of mate preference, relationship satisfaction, loneliness and problematic pornography use between China and Hungary were analyzed. The results showed that the model was more suitable for Chinese population, and its fitting indexes were $\mathrm{CFI}=0.932$, TLI $=0.921$ and $\mathrm{RMSEA}=0.059$. 
SY25-2

\title{
Same Same but Different: A Clinical Characterization of Men with Hypersexual Disorder in the Sex@Brain-Study
}

\author{
Jannis Engel ${ }^{*}$, Maria Veit ${ }^{1}$, Christopher Sinke ${ }^{1}$, Ivo-Aleksander Heitland ${ }^{1}$, Jonas Kneer ${ }^{1}$, \\ Thomas Hillemacher ${ }^{2}$, Uwe Hartmann ${ }^{1}$, Tillmann H. C. Krueger ${ }^{l}$ \\ ${ }^{1}$ Hannover Medical School \\ ${ }^{2}$ Klinikum Nuremberg \\ engel.jannis@mh-hannover.de
}

Problems arising from hypersexual behavior are often seen in clinical settings. We aimed to extend the knowledge about the clinical characteristics of individuals with hypersexual disorder (HD). A group of people who fulfilled the proposed diagnostic criteria for HD (Men with HD, $n=50)$ was compared to a group of healthy controls $(n=40)$. We investigated differences in sociodemographic, neurodevelopmental, and family factors based on self-report questionnaires and clinical interviews. Men with HD reported elevated rates of sexual activity, paraphilias, consumption of child abusive images and sexual coercive behavior compared to healthy controls. Moreover, rates of affective disorders, attachment difficulties, impulsivity and dysfunctional emotion regulation strategies were higher in men with HD. Men with HD seem to have experienced various forms of adverse childhood experiences, but there were no further differences in sociodemographic, neurodevelopmental factors and family factors. Regression analyses indicated that attachment-related avoidance and early onset of masturbation differentiated between men with HD and healthy controls. In conclusion, men with HD appear to have the same neurodevelopment, intelligence levels, sociodemographic background, and family factors compared to healthy controls, but they report different and adverse experiences in childhood, problematic sexual behavior and psychological difficulties.

\section{SY25-3}

\section{The distracting effect of pornographic pictures on working memory performances in hypersexual disorder}

\author{
Maria Veit ${ }^{1 *}$, Maria Veit ${ }^{1}$, Christopher Sinke ${ }^{1}$,Jannis Engel ${ }^{1}$, Jonas Kneer ${ }^{1}$, Uwe \\ Hartmann $^{1}$, Thomas Hillemacher ${ }^{2}$, Tillmann H. C. Krueger ${ }^{1}$ \\ ${ }^{1}$ Hanover Medical School \\ ${ }^{2}$ Klinikum Nuremberg \\ veit.maria@mh-hannover.de
}

Hypersexual disorder (HD) comes with clinically significant personal distress for patients and further social and medical consequences. Emerging evidence suggests impairments in executive functioning might be related to symptoms of hypersexual disorder. Findings from our "Sex@brain” study on male hypersexuality are presented. Here, a clinically character- 
ized sample of male participants with HD $(n=50)$ is compared with healthy volunteers (n $=40$ ) who participated in a classical fMRI n-back working memory task with distracting sexually explicit stimuli. Men with HD showed diminished working memory performances in the distracting sexually explicit condition. These performances were in line with altered functional activations.

\title{
SY25-4
}

\section{Comparison of effects of pharmacological and psychological treatment of CSBD}

\author{
Mateusz Gola ${ }^{1 *}$, Michal Lew-Starowicz ${ }^{2}$, Malgorzata Draps ${ }^{1}$, Ewelina Kowalewska ${ }^{1}$ \\ ${ }^{1}$ Clinical Neuroscience Laboratory, Institute of Psychology, Polish Academy of Sciences, Poland \\ ${ }^{2}$ Institute of Psychiatry and Neurology, Poland \\ mgola@ucsd.edu
}

Background and aims: Very little is known about the effectivity of various forms of CSBD treatment. Here we compared effects of pharmaceutical treatment with paroxetine, naltrexone and placebo.

Methods: Seventy-four males seeking treatment for CSBD and meeting CSBD criteria (as proposed in ICD-11) were recruited for the study and randomly assigned into the paroxetine $(\mathrm{n}=24)$, naltrexone $(\mathrm{n}=25)$ or placebo $(\mathrm{n}=25) 5$-month long treatment.

Results: Drop-out ratio in placebo condition was significantly $(\mathrm{p}<0.05)$ higher than in naltrexone and paroxetine. Results during the pre and post assessments with Brief Pornography Use Screener, and Hypersexual Behavior Inventory indicates significant improvement for all conditions: paroxetine, naltrexone and placebo $(\mathrm{p}<0.01)$, however ecological momentary assessment of craving for pornography watching indicates significantly higher decrease for paroxetine and naltrexone, than placebo $(\mathrm{p}<0.01)$.

Conclusions: Paroxetine and naltrexone can be effective in treatment of CSBD, especially in reduction of craving for problematic pornography use.

\section{SY25-5}

\section{Promoting sexual health through integrative sexual wellness education}

\author{
Gretchen Blycker* \\ University of Rhode Island \\ gblycker@uri.edu
}

College-aged young adults may be at increased risk for experiencing sexual assault, contracting sexually transmitted infections, viewing pornography problematically and engaging in compulsive sexual behaviors. As such, integrative sexual wellness education that promotes respect-based sexuality may decrease risks for sexual-related harms. In this presentation, 
I will draw on experiences teaching human sexuality to college students, with a focus on describing how students may engage in a process of self-awareness and mindfulness-based practices in the cultivation of sexual intelligence, positive sexual self-esteem, sexual empathy, and connection with their authentic sexual selves. The educational utility of the Mindful Model of Sexual Health may provide a personalized process for students to use as a framework to organize information regarding sexual and relational health. Using this framework, students may bring awareness and insight into their sexuality and address specific barriers to healthy functioning. The eight dimensions of this model (biological health, sexual-emotional health, individuation, intimacy, communication, self-awareness, spirituality, and mindfulness) represent components of an interrelated system organized toward health, balance, healing, and satisfaction. Other considerations (for example, how the model may help address sexual concerns or uncertainties in the current technological environment that young adults often encounter) will be discussed.

\title{
SY26-1
}

\section{Self-exclusions in online poker gambling: efficacy as compared to matched controls}

\author{
Amandine Luquiens ${ }^{1 *}$, Henri-Jean Aubin ${ }^{2}$, Amine Benyamina ${ }^{3}$, Stephane Gaiffas ${ }^{4}$, \\ Emmanuel Bacry
}

${ }^{1}$ APHP, CESP-Inserm U1178, Univ Paris Sud, Ecole Polytechnique Paris CMAP, France

${ }^{2}$ APHP, CESP-Inserm U1178, Univ Paris Sud, France

${ }^{3}$ APHP, Univ Paris Sud, Equipe d'accueil PSYCHOMADD, France

${ }^{4}$ Univ Paris Diderot, Ecole Polytechnique Paris CMAP, France

${ }^{5}$ Univ Paris Sud, Ecole Polytechnique Paris CMAP, France

amandineluquiens@gmail.com

Background: The aim of this study was to assess the efficacy of self-exclusion as compared to matched controls.

Method: We included all gamblers who first self-excluded over a 7-year period $(\mathrm{n}=4887)$ on a poker website, and gamblers matched on sex, age and account age ( $\mathrm{n}=4451)$. We compared between group money and time spent with mixed models. Analyses were performed on the whole sample, on the sub-groups with the highest time or money involvements and on highly involved short self-excluders ( $<90$ days). Effect sizes were calculated at 12 months among the sub-groups.

Results: A significant effect was found on money and time spent over the 12 months after the end of the self-exclusion period. Among the high financially involved gamblers, no significant change was found on money spent and effect size was low (0.18). Among the high temporally involved gamblers, significant change was found on time spent and effect size was moderate (0.34). No significant effect of short self-exclusion was found on the most involved gamblers, and effect sizes were low for money $(0.17)$ and negative for time $(-0.1)$.

Conclusions: Self-exclusion seems efficient at 12 months. However, efficacy of short selfexclusion should be further explored among the most involved gamblers. 


\title{
SY26-2
}

\section{Five-year follow-up of a sample of gamblers: predictive factors of relapse}

\author{
Marie Grall-Bronnec ${ }^{2}$, Jean-Benoit Hardouin ${ }^{2}$, Jeu Group, Gaelle Challet-Bouju ${ }^{l}$ \\ ${ }^{1}$ UMR 1246 - Universite Nantes / Addictology Department - CHU Nantes \\ ${ }^{2} \mathrm{UMR} 1246$ - Universite Nantes \\ marie.bronnec@chu-nantes.fr
}

There are few studies of the long-term evolution of gambling disorder (GD). The aim of this study was to identify factors that could predict relapse in GD.

Data were part of a dataset of a large 5-year cohort of gamblers (with or without GD). Participants were evaluated at inclusion and each year using a structured clinical interview and self-report questionnaires. For this specific study, inclusion criteria were: (i) transiting from GD to remission at a time of the follow-up; (ii) undergoing at least one follow-up visit after. Markov model-based approach was employed to examine predictive factors associated with relapse at the next follow-up visit.

A total of 87 gamblers were included. Forty-nine of them remained in remission, whereas 38 relapsed. The risk of relapsing decreased for participants who have experienced at least one month of abstinence, and for those having a high score at the TCI-Cooperativeness at the previous follow-up visit. In contrast, the risk of relapsing increased for participants with a current mood disorder diagnosed at the previous follow-up visit.

Our findings suggest the existence of predictive factors of relapse in remitted gamblers. These results lead to think about adaptive care to promote long-term remission.

\section{SY26-3}

\section{Current Trends of Online Gambling Disorder and Clinical Experiences in Korea}

\author{
Keun Ho Joe*
}

National Center for Mental Health of Korea

knozodr@hanmail.net

Most of the gamblers who visit gambling disorder clinic in Korea are entrapped by the online gambling. Among the online gambling, the most serious problem is "Sports toto". Even though there is the "Proto" that is legalized site operated by the Korea Sports Promotion Foundation (KSPO), most of the gambling addicts usually accessed other illegal sites by smartphone or PC. Because there are no restrictions or regulation of the illegal toto sites, many gamblers suffered from their bad credit. Furthermore, there are no limitations of access age, the gamblers experienced the first illegal toto when they were high school students. 
Currently, the young gamblers insisted that their main problems are related to new types of betting. For example, the "even and odd" game, "graph" game, and their variants. The common features of these novel games are simple and instant. These games are illegal of course, the gamblers can bet unlimitedly and throw away their entire properties in a twinkle.

I will introduce 3 young adult patients who are suffered from gambling problem. Their problem started at late teenager or early adulthood but already they became delinquent borrowers. Despite the problems were catastrophic, the patients showed gradual improvement.

\title{
SY26-4
}

\section{Effectiveness of CBT-based outpatient treatment program for gambling disorder: multi-study site randomized control trial in Japan}

\author{
Takanobu Matsuzaki*, Sachio Matsushita, Kotaro Nishimura, Satoshi Furuno, \\ Hitomi Okada, Susumu Higuchi \\ Kurihama Medical and Addiction Center \\ takanobum@gmail.com
}

Background: According to a recent national survey, the estimated prevalence of lifetime gambling disorder (GD) was 3.6\% in Japan. Despite major associated health and social problems, a standardized treatment program for GD has yet to be developed. The purpose of this study is to develop a cognitive behavioral therapy (CBT)-based outpatient treatment program and to evaluate its efficacy using a randomized control trial (RCT).

Methods: All study participants were aged between 20 and 65 years old and were recruited via website screening. Subjects met the DSM-5 diagnostic criteria for GD. 187 participants were enrolled and randomly assigned to either the intervention or waiting (control) groups. The intervention group underwent six sessions of a CBT-based program every 2 weeks at 35 treatment facilities throughout Japan. The endpoints of this study were: frequency of gambling and sum of money wagered. Follow-up of participants continued for 6 months. In the symposium, the content of the program and the result of the RCT will be presented and discussed. 


\title{
SY27-1
}

\section{Gray matter volume abnormalities in compulsive sexual behavior, gambling and alcohol use disorder}

\author{
Mateusz Gola ${ }^{1 *}$, Matgorzata Draps ${ }^{1}$, Guillaume Sescousse ${ }^{2}$, Artur Marchewka ${ }^{3}$, \\ Jacek Matuszewski ${ }^{3}$, Agnieszka Duda ${ }^{4}$, Michal Lew-Starowicz ${ }^{5}$, Maciej Kopera ${ }^{6}$, \\ Andrzej Jakubczyk6, Marcin Wojnar ${ }^{6}$ \\ ${ }^{1}$ Clinical Neuroscience Laboratory, Institute of Psychology, Polish Academy of Sciences, Poland \\ ${ }^{2}$ Lyon Neuroscience Research Center, France \\ ${ }^{3}$ Laboratory of Brain Imaging, Nencki Institute of Experimental Biology, Polish Academy of \\ Sciences, Poland \\ ${ }^{4}$ Krakowskie Stowarzyszenie Terapeutow Uzaleznien, Cracow, Poland \\ ${ }^{5}$ Institute of Psychiatry and Neurology, Poland \\ ${ }^{6}$ Department of Psychiatry, Medical University of Warsaw, Poland \\ mgola@psych.pan.pl
}

Background and aims: comparing gray matter volume between patients with Compulsive Sexual Behavior Disorder (CSBD), Gambling Disorder (GD), Alcohol Use Disorder (AUD) and healthy controls (HC).

Methods: MRI data were acquired from: 26 CSBD, 26 for GD, 21 for AUD and 25 age matched HC. Severity of CSBD was assessed by SAST-R and BPS, GD by SOGS and AUD by AUDIT. Healthy controls scored below the pathological thresholds for all these tests. Subjects with history of other psychiatric disorders and comorbid addiction were excluded. Voxel-Based Morphometry (VBM) analysis was conducted to assess differences in brain volumetry.

Results: Compared with HC, addicted individuals (CSBD, GD and AUD all together vs $\mathrm{HC}$ ), showed a significantly less gray matter volume in the left frontal pole (peak: $\mathrm{x}=-29, \mathrm{y}$ $=62, \mathrm{z}=2$, $\mathrm{t}$-stat $=4.46$ ). The most pronounced atrophy was observed in the AUD and GD groups, and the least pronounced in the CSBD group. We did not find any significant correlation between symptoms measured by questionnaires and volume of specific brain regions in specific group of patients.

Conclusions: Loss of brain volume in PFC has been consistently reported in substance addictions (such as AUD), but there has been a lack of consistent results in the case of GD and no data in CSBD. Our research is the first showing similar decrease of PFC volume across CSBD, GD and AUD patients with no comorbid addictions. 
SY27-2

\title{
Recent Findings in the Neurobiology of Behavioral Addictions
}

\author{
Marc N. Potenza* \\ Yale University \\ marc.potenza@yale.edu
}

The precise diagnostic entities classified as addictions has been debated. Although substanceuse disorders have for decades been considered the prototypical addictions, the extent to which certain subtances may be considered as addictive has changed throughout the past century (e.g., with respect to tobacco). Although food (or certain types of foods) have been proposed as addictive, debate has occurred regarding the concept and clinical utility of food addiction. Binge eating disorder, introduced into the main text of the DSM in its fifth edition, has been proposed to share particular similarities with food addiction. Another condition, gambling disorder, was classified together with substance use disorders in DSM-5, with a similar reclassification observed in ICD-11. Here, we will present neuroimaging findings suggesting both similarities and differences across these conditions, with a focus on cocaine use, binge eating and gambling disorders. Implications for prevention and treatment development will be discussed.

\section{SY27-3}

\section{Alteration of functional connectivity in patients with gaming disorder comorbid with other psychiatric disorders}

\author{
Shinya Kuriki ${ }^{*}$, Susumu Higuchi ${ }^{2}$, Hideki Nakayama², Satoko Mihara², \\ Yasuomi Okazaki ${ }^{3}$, Yumie Ono ${ }^{4}$, Hiroshi Kobayashi ${ }^{5}$ \\ ${ }^{1}$ Tokyo Denki University, Hokkaido University \\ ${ }^{2}$ Kurihama Medical and Addiction Center \\ ${ }^{3}$ Waseda University \\ ${ }^{4}$ Meiji University \\ ${ }^{5}$ Tokyo Denki University \\ kuriki_ms@hotmail.com
}

Recent studies on Internet Gaming Disorder (IGD) have addressed the influence of comorbid disorders on neurobiological activities in IGD people. Resting-state (rs-) fMRI is a beneficial method applicable to young patients, requiring no tasks during examination. We have investigated functional connectivity (FC) using rs-fMRI for patients of 12-26 years of age diagnosed with IGD $(n=26)$ and IGD having comorbid conditions $(n=20)$, and healthy control (HC) individuals $(n=30)$ of the similar age range. Analyses of FC were based on a data-driven method using permutation tests to find core nodes having a sizable cluster (Family-Wise Error $<0.05$ ) of altered-FC connections with other brain areas. Obtained results indicated that GD patients had reduced FC compared with $\mathrm{HC}$ in dorsolateral prefrontal areas 
connected to a limbic core node and primary and associated sensory / motor areas connected to paracentral core nodes. In GD patients having comorbid ASD and ADHD, wide-range core nodes were identified, in comparison with GD-only patients, in the prefrontal cortex, temporal pole, parietal cortex, and limbic areas, which were not overlapped with the GD-only core nodes. Connections of reduced FC to these core nodes existed in dorsolateral / orbital prefrontal areas, amygdala, basal ganglia and cingulate, suggesting impaired reward function with comorbidity of ASD / ADHD.

\title{
SY27-4
}

\section{Neural mechanisms of Craving Behavioral Therapy in treating Internet Gaming Disorder}

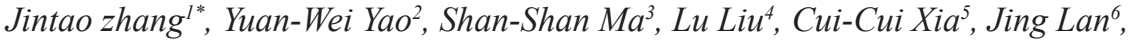 \\ Lu-lu Wu ${ }^{1}$, Xiao-Yi Fang ${ }^{6}$, Marc N Potenza ${ }^{7}$
}

\author{
${ }^{1}$ State Key Laboratory of Cognitive Neuroscience and Learning and IDG / McGovern Institute for \\ Brain Research, Beijing Normal University, Beijing, China \\ ${ }^{2}$ Einstein Center for Neurosciences Berlin, Chariteplatz 1, 10117, Berlin, Germany; State Key \\ Laboratory of Cognitive Neuroscience and Learning and IDG / McGovern Institute for Brain \\ Research, Beijing Normal University, Beijing, China \\ ${ }^{3}$ Institute of Developmental Psychology, State Key Laboratory of Cognitive Neuroscience and \\ Learning and IDG / McGovern Institute for Brain Research, Beijing Normal University, Beijing, \\ China \\ ${ }^{4}$ German Institute of Human Nutrition Potsdam-Rehbruecke, 14558 Nuthetal, Germany; State Key \\ Laboratory of Cognitive Neuroscience and Learning and IDG / McGovern Institute for Brain \\ Research, Beijing Normal University, Beijing, China \\ ${ }^{5}$ Students Counseling Center, Beijing Normal University, Beijing 100875, China \\ ${ }^{6}$ Institute of Developmental Psychology, Beijing Normal University, Beijing 100875, China \\ ${ }^{7}$ Department of Psychiatry and Neuroscience, Child Study Center, Yale University School of \\ Medicine, Connecticut Mental Health Center, New Haven, Connecticut Council on Problem \\ Gambling, Wethersfield, CT, USA \\ zhangjintao@bnu.edu.cn
}

Although behavioral interventions predominate in the treatment of Internet Gaming Disorder (IGD), the neural mechanisms underlying their efficacy in IGD remain unclear. Thus, in this study, we investigated how corticostriatal circuitry, a target of behavioral interventions in drug addictions, responded to a behavioral interventions in IGD. Greater resting-state functional connectivity (rsFC) of the ventral striatum (VS) to left inferior parietal lobule (IIPL), right inferior frontal gyrus and left middle frontal gyrus was positively associated with IGD severity cross-sectionally. A portion of participants attended a follow-up craving behavioral intervention (CBI) study, in which 20 IGD subjects received the $\mathrm{CBI}(\mathrm{CBI}+)$, compared to 16 IGD subjects did not (CBI-). CBI + versus CBI- participants showed a greater decrease in VS-IIPL connectivity, along with decreases in addiction severity and craving. CBI + participants also demonstrated significantly reduced resting-state functional connectivity between the: (1) orbital frontal cortex with hippocampus / parahippocampal gyrus; and, (2) posterior 
cingulate cortex with supplementary motor area, precentral gyrus, and postcentral gyrus. During an fMRI cue reactivity task, the CBI + group showed enhanced activation in the right insula and decreased insular connectivity with the lingual gyrus in a treatment-sensitive manner. These results together provide insight into possible ways to enhance treatment efficacy for IGD.

\title{
SY27-5
}

\section{Neuropsychological and neurobiological correlates of specific Internet- use disorders}

\author{
Matthias Brand ${ }^{1}$, Elisa Wegmann ${ }^{1}$, Marc N. Potenza $^{2}$ \\ ${ }^{1}$ University of Duisburg-Essen \\ ${ }^{2}$ Yale University School of Medicine \\ matthias.brand@uni-due.de
}

Several neuroscientific theories and models explain addictive behaviors (e.g. Incentive-Sensitization-Theory, Robinson \& Berridge, 2008; dual-process approaches, Everitt \& Robbins, 2005). Based on these, the Interaction-of-Person-Affect- Cognition-Execution (I-PACE) model of specific internet-use disorders (IUD) (Brand et al., 2016) has been suggested. The progression of an imbalance between increasing incentive-oriented urges and decreasing inhibitory control over these urges and desires is considered important for the development and maintenance of specific IUD. Reductions in executive functions are considered both predisposing variables and consequences of addictive behaviors. In gaming disorder and other specific IUD, such as pornography-use disorder and online-buying-shopping disorder, specific inhibitory control processes are considered being affected by craving in the context of the confrontation with addiction-related stimuli. This interaction likely contributes to habitual behaviors in individuals with IUD. Consistent with these theories, current meta-analyses of neuroimaging studies on gaming disorder (and partly on pornography-use disorder) have shown the involvement of hyperactive limbic structures including the ventral striatum and hypoactive prefrontal-striatal circuits involved in cognitive control over the behavior. Emotion- / impulse-driven aspects and inhibitory control should be addressed synergistically in research on IUD and in clinical practice. 
SY28-1

\title{
Alteration of neural activity related to social interaction in adolescents with excessive social media use
}

\author{
Dai-Jin Kim*, Jiwon Chun, Min Kyung Hu \\ Department of Psychiatry, The Catholic University of Korea College of Medicine \\ cuneus@naver.com
}

In this study, we aim to identify altered brain connectivity associated with excessive internet use, and to investigate correlations between depressive symptoms and frontostriatal connectivity. We focused on investigating functional connectivity in the caudate nucleus (Cau), anterior cingulate cortex (ACC), and medial prefrontal cortex (MPFC), which is related to cognitive control. We analyzed data from 40 adolescents with excessive internet use (IA) and 42 healthy controls (HC).

In IA group compared with $\mathrm{HC}$, we observed lower functional connectivity between the right $\mathrm{Cau}$ and ACC. In addition, we observed that Adolescents with IA showed negative correlation between depression and functional connectivity between caudate nucleus and MPFC. Furthermore, there were significant correlations between frontostriatal connectivity of Cau-ACC and depression, and between frontostriatal connectivity of MPFC-ACC and depression in adolescents with IA.

The current study suggests that adolescents with IA had altered functional connectivity in the frontostriatal region. In particular, the weaker functional connectivity between the Cau and MPFC IA showed, the higher depression they revealed. Moreover, the higher depression they revealed, the weaker functional connectivity between the Cau and MPFC in IA compared to HC. Excessive internet use might appear to elicit depressive symptom, and this psychological change may affect frontostriatal connectivity.

\section{SY28-2}

\section{Error processing deficit and neurophysiological correlates in Internet gaming disorder}

\author{
Jung-Seok Choi", Minkyung Park, Ji Yoon Lee, Sun Ju Chung, Bomi Kim, A Ruem Choi \\ SMG-SNU Boramae Medical center \\ reneedrv@gmail.com
}

The ability to detect and correct errors is a critical aspect of human cognition. Neuronal dysfunction in error processing has been reported in addictive disorders. The aim of this study was to investigate neural systems underlying error processing using event-related potentials (ERPs) and current source localization as well as neurocognitive executive function tests in patients with Internet-gaming disorder (IGD). A total of 68 individuals (34 patients with IGD and 34 healthy controls (HCs)) were included, and two ERP components, error-related negativity (ERN) and error positivity $(\mathrm{Pe})$, were extracted during a GoNogo task. Patients 
with IGD exhibited significantly reduced ERN and Pe amplitudes compared with HCs. Standardized low resolution brain electromagnetic tomography (sLORETA) in between-group comparisons revealed that patients with IGD had decreased source activations of the Pe component in the anterior cingulate cortex under the Nogo condition. These ERP changes were associated with executive function deficits in IGD patients. The results suggest that IGD may be associated with alterations in neural activity related to both the early unconscious and the later conscious stages of error processing, as well as deficits in executive function. Thus, deficits in error processing could be considered a candidate neurophysiological marker for IGD.

\title{
SY28-3
}

\section{Prediction of internet gaming disorder based on integrative analysis of EEG and PET data}

\author{
Donghwan Lee ${ }^{1 *}$, Boram Jeong ${ }^{1}$, Ji Yoon Lee ${ }^{2}$,Heejung Kim², Jung-Seok Choi, \\ Youngjo Lee Le $^{3}$ \\ ${ }^{1}$ Ewha Womans University \\ ${ }^{2}$ SMG-SNU Boramae Medical Center \\ ${ }^{3}$ Seoul National University \\ donghwan.lee@ewha.ac.kr
}

Internet gaming disorder (IGD) becomes an important issue and is one of the most discussed psychosocial problems. To prevent IGD and provide appropriate intervention, an accurate prediction method for identifying IGD is necessary. In many works of literature, it is discovered that brain-related indicators such as Electroencephalography and Positron Emission Tomography, and the other clinical features can be associated with IGD. In this study, we propose an IGD prediction method by integrating all three modalities to enhance prediction accuracy. In such multimodal data, each modality has its own source, and its characteristics are different. Unlike the conventional methods which usually concatenate all features into one feature vector, we adopt a multiple-kernel support vector machine (SVM) to identify the IGD patient. By using real data collected from Korean adults, we compare the prediction performance of standard machine learning methods such as SVM, random forest, boosting with the proposed method. We show that the prediction accuracy of the optimal multiple-kernel SVM was much higher than other standard methods. Furthermore, we figure out the contribution of each modality in the prediction model through the kernel weights. 
SY28-4

\title{
Personalized treatment using smart health care system for smartphone addiction
}

\author{
In Young Choi ${ }^{1 *}$, Mun Joo Choi ${ }^{1}$, In Hye Yook ${ }^{1}$, Sun Jung Lee ${ }^{1}$, Soo Kyung Yoon ${ }^{2}$, \\ Hyuck Jun Jeong ${ }^{2}$, Dai-Jin Kim ${ }^{3}$ \\ ${ }^{1}$ Department of Medical Informatics, College of Medicine, The Catholic University of Korea, Seoul, \\ Republic of Korea 2. Department of Biomedicine \& Health Sciences, College of Medicine, \\ The Catholic University of Korea, Seoul, Republic of Korea \\ ${ }^{2}$ Department of Medical Informatics, College of Medicine, The Catholic University of Korea, Seoul, \\ Republic of Korea \\ ${ }^{3}$ Department of Psychiatry, Seoul St. Mary's Hospital, College of Medicine, The Catholic University \\ of Korea, Seoul, Republic of Korea \\ cmj7705@naver.com
}

A smart health care system provides personalized treatment based on the patient's condition by checking it in real time. With the advent of a variety of personal health devices and proliferation due to the development of Internet of Things make it easier to collect and managing the health data. In this study, proposed developed smartphone application based on the smart health care system which aim for personalization of individual patient's treatment, purpose to prevent overuse of smartphones. The smart health care system for mental health and smartphone addiction care, which is called as 'Mindscare', aims to prevent smartphone addiction by providing personalized health care services. This system combined with traditional treatment and smart health care treatment. Traditional treatment approaches with MET (Motivational Enhancement Therapy), CBT (Cognitive Behavioral Therapy), CM (Contingency Management), SDT (Self Determination Theory) and computerized those treatments as the systematic interventions. Also, we developed the web monitoring system which received mindscare health data every 10 minutes. Through the monitoring patient's data from the web, the treatment expert provides personalized intervention individually.

This study is to demonstrate the systematic protocol and interventions. As empirical study is under progressing, we expect meaningful results obtained, if we proceed the study accordingly. 
SY30-1

\title{
Structural Brain Changes Associated with Extensive Video Gaming
}

\author{
Qinghua He ${ }^{1 *}$, Ofir Turel ${ }^{2}$, Lei Wei ${ }^{1}$, Antoine Bechara ${ }^{3}$ \\ ${ }^{1}$ Southwest University \\ ${ }^{2}$ California State University, Fullerton \\ ${ }^{3}$ University of Southern California \\ heqinghua@gmail.com
}

Videogames are a popular form of entertainment; in 2019 there are about 2.5 Billion gamers worldwide. The present study aimed to understand the possible brain alterations that are linked to extensive video game play (defined in this study as about $3 \mathrm{~h} /$ day). Fifty-two volunteers were recruited with 26 extensive LOL players and 26 people who have not ever played LOL and have not played videogames in the last six months. Structural brain neuroimaging data were compared between groups with Voxel Based Morphometry and cortical thickness analyses. The VBM results showed that extensive gamers have reduced gray matter volume in the VMPFC bilaterally, and especially the right VMPFC, as well as in left DLPFC extended to the LFP. The cortical thickness analysis demonstrated that extensive gamers had several thinner cortices compared to non- gamers. Importantly, these included the L SPL and several prefrontal regions, including the L VMPFC, bilateral DMPFC and L DLPFC. These results showed that less efficient prefrontal regions in terms of inhibition in extensive gamers, as reflected in lower volumes of, and thinner VMPFC and DLPFC. We call for future research to further examine extensive video gamers and the potential for video games to generate desired and unwanted brain changes.

\section{SY30-2}

\section{Impulsivity as a marker of vulnerability to gambling disorder: a study of affected siblings}

\author{
Eve H Limbrick-Oldfield ${ }^{*}$, Inge Mick², Rachel E Cocks², Remy SA Flechais ${ }^{2}$, \\ Samuel Turton ${ }^{2}$, Anne Lingford-Hughes ${ }^{2}$, Henrietta Bowden-Jones ${ }^{2}$, Luke Clark ${ }^{1}$ \\ ${ }^{1}$ University of British Columbia \\ ${ }^{2}$ Imperial College London \\ eve@psych.ubc.ca
}

Psychological markers in individuals with gambling disorder could reflect transdiagnostic vulnerability to addiction or neuroadaptive consequences of long-term gambling. Using an endophenotypic approach to identify vulnerability markers, we tested biological relatives of cases with gambling disorder. Male participants seeking treatment for gambling disorder $(n=20)$ were compared with a male control group $(n=18)$. Biological siblings of cases with 
gambling disorder ( $\mathrm{n}=17$, unrelated to the gambling disoder group) were compared with a partially-overlapping control group $(\mathrm{n}=19)$. Participants completed a comprehensive assessment of clinical scales and neurocognitive functioning. Consistent with previous literature we observed a behavioural and cognitive profile in the gambling disorder group that included elevated levels of self-report and behavioural impulsivity, and increased risk-taking on the Cambridge gamble task. We did not observe impaired motor impulsivity on the the stop-signal task. Siblings of cases with gambling disorder showed some similar effects, with elevated levels of negative urgency (impulsivity) and increased risk-taking on the Cambridge gamble task, suggesting these may represent a pre-existing vulnerability to gambling disorder.

\section{SY30-3}

\section{Examining neural reactivity to gambling cues in the age of sports betting}

Damien Brevers $^{1 *}$, Guillaume Sescousse ${ }^{2}$, Pierre Maurage ${ }^{3}$, Joel Billieux $^{1}$

${ }^{1}$ University of Luxembourg

${ }^{2}$ University of Lyon

${ }^{3}$ UCLouvain

damien.brevers@uni.lu

The goal of this presentation is to provide new insights as to how and why functional magnetic resonance imaging (fMRI) research on gambling cue reactivity can contribute to significant progress towards the understanding of gambling disorder. After having offered an overview of experimental paradigms and past findings related to gambling cue reactivity, we will suggest methodological avenues for future research. Throughout this talk, we will see that the fMRI literature on problem gambling has identified the main neural pathways associated with reactivity to gambling cues. Yet, the current knowledge on the key factors underlying cue reactivity in gambling is still very incomplete. Here, we suggest that the recent expansion of online sports betting calls for a new line of research offering a fine-grained and up-to-date approach of neural cue reactivity in gambling disorder. More specifically, we advance that experimental designs manipulating individual-specific and study- specific factors related to sports betting have the potential to foster progress towards efficient treatment and prevention of gambling disorder. 
SY31-1

\title{
Measures against substance use disorders and gambling disorders in Japan
}

\author{
Tomomi Toyama*, Akimori Mizoguchi \\ Ministry of Health, Labour and Welfare \\ tooyama-tomomi@mhlw.go.jp
}

Recently, there have been increased interests in substance use disorders and gambling disorders in Japan. Also, it strongly requires measures against social problems related to those disorders, such as poverty, violence and debt. While there are various measures in the world, for examples medical care, recovery support, regulation, and prevention educaion, in this session, we firstly would like to make a presentation focused on recent actual efforts in Ministry of Health, Labour and Welfare in Japan against those problems. The second, with the experts and private support groups of substance use and gambling disorders, we would like to have some discussions about the way of upgrades of effective measures meeting for the needs of communities.

\section{SY31-2}

\section{The Role of General Psychiatric Hospitals in Gambling Disorder}

\author{
Toshiaki Tsuneoka ${ }^{1 *}$, Hideaki Kawai ${ }^{2}$, Sachiko Yokoyama ${ }^{2}$, Naoko Fujisawa ${ }^{2}$, Wakaho \\ Hayashi², Sumiko Nakamura ${ }^{3}$ \\ ${ }^{1}$ Showa University School of Medicine, Department of Psychiatry \\ ${ }^{2}$ Showa University \\ ${ }^{3}$ KOMAGINO Hospital \\ tsune1027@hotmail.com
}

Gambling disorder (GD) is disabling and associated with various psychiatric, financial and relational problems. Recently GD has garnered increased attention, but there still are only a few hospitals specialized for its treatment in Japan. Therefore, it is important to consider how general psychiatric hospitals can take roles in GD treatment.

While anti-alcohol drugs exist to treat alcohol addiction, there is no such medication for GD, and treatments often rely on the use of self-help groups such as Gamblers Anonymous or Gamano.

Still, general psychiatric hospitals can take important roles in treating GD. First, they can provide diagnosis, allowing patients to recognize the condition as treatable and increasing their motivation to improve their situation. Comorbid conditions such as attention deficit hyperactivity disorder or bipolar disorder can also be detected and pharmacotherapy on the comorbid conditions may also improve the GD. Second, they may prevent suicide attempts; GD is strongly associated with suicidal tendencies, and admitting suicidal GD patients may save lives and also provide opportunity for appropriate interventions. Thus, general psychiatric hospitals have potential to offer effective treatments for GD in collaboration with selfhelp groups. We will discuss ways to collaborate effectively in detail. 
SY31-3

\title{
Difficulties and supports for family members of people with gambling disorder in Japan
}

\author{
Nobuaki Morita ${ }^{1 *}$, Kiyomi Arai², Noriko Tanaka ${ }^{3}$, Yukiko Kawaguchi ${ }^{4}$ \\ ${ }^{1}$ Faculty of Medicine, University of Tsukuba \\ ${ }^{2}$ Tokyo Metropolitan University, Division of Nursing Sciences \\ ${ }^{3}$ The Society Concerned about the Gambling Addiction \\ ${ }^{4}$ Uekusa Gakuen University, Faculty of Child Development and Education \\ nobuakim@nifty.com
}

This research investigated difficulties of family members of people with gambling problems and their utilization of support services regarding these problems. Subjects were 224 family members who attended counseling offered by a private organization for recovery from gambling addiction. Most were wives and mothers of gamblers. The main kinds of gambles included pachinko / slot machines (92.0\%). Frequent gambling problems which family members experienced were economic difficulties (lifelong 86.1\%; now 33.1\%), family rifts / marital separation / divorce $(43.7 \% ; 23.1 \%)$, depression $(43.5 \% ; 7.5 \%)$. Of the family members, $80 \%$ had gamblers' debt and $16.5 \%$ of them paid more than $10,000,000$ yen. Among subjects, $44.2 \%$ consulted support facilities within four years of discovering gambling problems, but it took more than 10 years for $26.8 \%$ of them to seek help from such facilities. Multiple logistic model revealed significant factors of number of problems related to gambling, including "the time period since detection of gambling problems until consultation" (OR: 5.2) for more than four lifelong problems, "continuation of gambling" (OR: 9.9), and age of gamblers (OR of $20 \mathrm{~s}: 30-40 \mathrm{~s}: 50 \mathrm{~s}$ were $1: 10.0: 12.0)$ for more than one current problem. These findings imply that we should help family to consult to support facilities for members with gambling disorders earlier.

\section{SY31-4}

\section{Efforts at gambling addiction recovery facilities General Incorporared Association GRACE LORD}

\section{Fumitaka Ikeda*}

General Incorporated Association GRACE LORD

g.lord1224@mirror.ocn.ne.jp

General Incorporated Association GRACE LORD founded in 2015 is a specialized recovery facility for gambling addiction. Here, recoveries from gambling addiction have been providing peer support as staff, and since its establishment, we have supported the recovery and reentry into society of over 100 gambling addicts. This time we will present about the GRACE LORD recovery program, the social attributes of the user, the type of gambling that addicts, the continuity of the program, the social reversion rate and the recurrence rate. 


\title{
SY31-5
}

\section{The roles of non-governmental organizations and issues of cooperation.}

\author{
Noriko Tanaka $^{1 *}$, Toshihiko Matsumoto ${ }^{2}$ \\ ${ }^{1}$ Public Interest Incorporated Association The Society Concerned about the Gambling Addiction \\ ${ }^{2}$ National Institute of Mental Health, National Center of Neurology and Psychiatry \\ ricomomokei@gmail.com
}

At the The Society Concerned about the Gambling Addiction, in addition to our main function of helping affected parties and families with intervention and support, we also work to help enlighten society, provide preventative education, creating partnerships and receptacles in regions throughout Japan and we are also involved in investigations / research.

I would like to report on the current situation and the problems we have seen through consultations, which includes the needs of families coming to see us and the difficulties they face. I would also like to touch on the difference in our role from that of government officials.

\section{SY32-1}

\section{Treatment Strategy of Internet Gaming}

\author{
Motohisa Katagami*
}

Department of Neuropsychiatry, Osaka City University Graduate School of Medicine motohisakatagami@gmail.com

The large availability of the internet and the embracing of new digital technologies like smartphones are changing people's way of life and introducing new social dynamics. Especially, there are many youths who have problems with the excessive use of the Internet Gaming. Internet game usage and gaming money has been rapidly increasing worldwide. Internet Gaming Disorder (IGD) has become a major social problem and important research topic. The World Health Organization (WHO) has set a new category named "Gaming Disorder" for the 11th Revision of the International Classification of Diseases (ICD-11).

There are many young IGD patients being consulted, but in many cases they are poorly motivated for treatment regardless of enthusiasm of their parents. They often do not want to see a doctor or the consultation drops off, and they become socially withdrawn. In order to manage IGD, the initial goal of the clinician is to identify the causes and negative consequences of excessive gaming and to focus on psychological factors of IGD.

At Osaka City University Medical School Hospital, medical department for Internet Gaming Disorder was established in 2012. We will report about treatment strategy of IGD at our hospital. 
SY32-2

\title{
Development of Intervention for Problematic Smartphone Use among Korean Adolescents: Using Mixed Methodology
}

\author{
JongSerl Chun* \\ Ewha Womans University \\ jschun@ewha.ac.kr
}

The purpose of this study is to develop smartphone addiction program for adolescents using mixed methods and to examine its effectiveness through pilot testing. In order to develop this program, three large clusters of methodology have been applied: 1) systematic review and survey, 2) concept mapping, 3) pilot test. At the first stage, systematic review on smartphone addiction program, and survey on the prevalence of smartphone addiction and its influencing factors have been conducted. According to the literature review and the survey result, group counseling and self-control was found to be the most effective program and influencing factor to internet / smartphone addiction among adolescents. To consolidate these findings, 5 main clusters from concept mapping (e.g., involuntary restriction, self-awareness and self-control, school factors, peer support, professional intervention program) and suggestions from the focus group interview were also taken into account. Having its basis on the cognitive-behavioral theory, finalized program consisted of 8 sessions. This program was proven to be effective among high-risk and potential-risk group of adolescents. By developing an effective smartphone addiction program for the Korean adolescents, it contributed to the development of evidence-based practice model in Korea.

\section{SY32-3}

\section{Serial multiple mediation effects of self-control and aggression in the relationship between Attention deficit hyperactivity disorder and Internet gaming addiction in adolescents using three-wave longitudinal data}

Hyeon Woo Yim*, Hyunsuk Jeong, Hyeon Woo Yim, Sun-Jin Jo, Seung-Yup Lee, Hae Kook Lee, Hye Jung Son, Ji Yun Choi

The Catholic University of Korea

suejeong@catholic.ac.kr

This study aims to investigate serial multiple mediation effect of self-control and aggression in the relationship between ADHD symptoms and risk of IGD in adolescents using 3 wave iCURE study. The study participants consisted of 1,754 secondary school students (male: 1,005 (57.3\%), female: 749 (46.7\%). We hypothesized that ADHD symptoms (T1) would indirectly influence development of risk of IGD (T3) through causally linked multiple mediators of self-control (T1) and aggression (T2). The model was tested for serial multiple 
mediation to see how they would be impact on each other. The total effect of ADHD (T1) on risk of IGD (T3) was significant ( $\beta=0.40,95 \%$ CI: 0.26-0.57). Serial-multiple mediation effects of self- control (T1) and aggression (T2), single mediation effect of self-control (T1), and single mediation effect of aggression (T2) were found to be statistically significant in the relationship between ADHD (T1) on risk of IGD (T3) $(\beta=0.044,95 \% \mathrm{CI}$ : $0.024-0.075, \beta=$ $0.09,95 \% \mathrm{CI}: 0.045-0.159$, and $\beta=0.046,95 \% \mathrm{CI}: 0.018-0.09$, respectively). These results may help us to see how ADHD symptoms (T1) and risk of IGD are linked and what the fundamental mediators are in the chain.

\title{
SY32-4
}

\section{Clinical characteristics of psychiatric comorbidity in gambling disorder in Japan}

\author{
Risa Yamada*, Kumi Miyashita ${ }^{1}$, Yusuke Tamura ${ }^{2}$,Takamitsu Hashimoto ${ }^{1}$, Kohji Takada , \\ Hisatsugu Miyata ${ }^{2}$ \\ ${ }^{1}$ Department of Psychology,Teikyo University \\ ${ }^{2}$ Department of Psychiatry, Jikei University School of Medicine \\ iuoriou3@gmail.com
}

The purpose of the present study is to investigate the involvement of the psychiatric comorbidity in the development of gambling disorder (GD). Subjects were 289 outpatients with GD (male / female: 264 / 25, average age: 40.0). The most popular gambling activity was pachinko $(n=215)$, followed by pachislo (Japanese slot machine; $n=145)$ and horse racing $(\mathrm{n}=60)$. The percentage of patients with psychiatric comorbidity was $67.3 \%$. The average of SOGS scores was 12.2. GD patients with mental disorders as the psychiatric comorbidity were 99 in number (depression, $\mathrm{n}=43$; attention deficit hyperactivity disorder ADHD, $\mathrm{n}=$ 22; bipolar disorder, $\mathrm{n}=10$; autistic spectrum disorders, $\mathrm{n}=9$, etc.). Those with substance use disorder were 105 in number (tobacco, $n=67$; alcohol, $n=43$ ), and those with behavioral addiction were 45 in number (creptmania, $\mathrm{n}=19$; sex-related behaviors, $\mathrm{n}=17$; excessive buying, $\mathrm{n}=13$ ). As for the relationship between the psychiatric comorbidity and GD, the comorbidity which was regarded to cause $\operatorname{GD}$ was $\operatorname{ADHD}(n=9)$, bipolar disorder $(n=4)$, depression $(n=3)$, and sex-related activities $(n=3)$, whereas the comorbidity which was regarded to be caused by GD was depression $(n=13)$, alcohol use disorder $(n=3)$, tobacco use disorder $(n=1)$, creptmania $(n=8)$, etc. The present study indicates a clinical significance of psychiatric comorbidity in GD. 


\title{
SY33-1
}

\section{Behavioral and Substance Addictions: Co-Occurrence and Association}

\author{
Sang-Kyu Lee*, Hui-Cheol Choi \\ Hallym University Medical Center, Chuncheon Sacred Heart Hospital \\ skmind@hallym.ac.kr
}

The occurrence of substance use disorders and behavioral addictions is quite common and this co-morbidity carries implications for the diagnosis and the treatment of these disorders. This study aimed at identifying the co-occurrence and associations among five different types of addiction (including 2 substances and 3 behaviors) in Korean adults. Screening tests assessing the severity and determining the high risk of each type of addiction were administered. To investigate the associations between different addictions, chi-square test and Fisher's exact test, associative analysis, and latent profile analysis were performed. There was a significant co-occurrence of several different types of addiction, and these relationships were considerably stronger in some subgroups than in others. The findings demonstrated a higher rate of co-occurrence of substance and behavioral addictions among males than among females. Individuals aged 20-29 years showed a very high rate of co-occurrence of five different types of addictions. Additionally, this study showed the close association between alcohol, nicotine, and gambling addictions. The most closely related pairs of addictions were internet and smartphone addiction. This result suggested that we need the development of targeted early therapeutic interventions and prevention strategies for co-morbid addiction problems and it will lead to improved treatments for comorbidity.

\section{SY33-2}

\section{Psychiatric comorbidities of ICD-11 gaming disorder}

\author{
Satoko Mihara*, Hideki Nakayama, Susumu Higuchi \\ National Hospital Organization Kurihama Medical and Addiction Center \\ mihara.satoko.pd@mail.hosp.go.jp
}

Background: It has been suggested that the rate of psychiatric and psychological comorbidities is high in individuals with DSM-5 Internet Gaming Disorder (IGD). However, studies focusing on Gaming Disorder (GD), as defined in the draft version of ICD-11, utilizing clinical cases are extremely limited. In this study, we report on the prevalence of comorbidities in patients with GD, who visited the specialist outpatient clinic at our center.

Methods: Subjects were 200 new outpatients who visited our specialist clinic between January and December 2018. They met the definition of GD. Psychiatric comorbidities were evaluated using MINI, SSAGA and LSAS-J and WAIS / WISC, based on face-to-face interviews conducted by clinical psychologists. Self-reported instruments evaluating for ADHD and autism spectrum disorder were also performed. 
Results: Nearly $50 \%$ of subjects possessed psychiatric comorbidities. ADHD showed the highest prevalence, followed by major depression. About one-third of the subjects were at risk for suicide. A 6-month follow-up study showed poorer outcome in subjects with comorbidities than those without comorbidities.

Conclusion: This study suggested that the psychiatric comorbidity rate of GD was relatively high and that comorbidities have important implications in the treatment of GD.

\title{
SY33-3
}

\section{Comorbid disorders as moderating factors in the treatment of internet- related disorders}

\author{
Kai W. Müller ${ }^{*}$, Michael Dreier ${ }^{1}$, Manfred E. Beutel ${ }^{2}$, Klaus Woellfling ${ }^{1}$ \\ ${ }^{1}$ Outpatient Clinic for Behavioral Addictions, University Medical Center Mainz, Germany \\ ${ }^{2}$ Department for Psychosomatic Medicine and Psychotherapy, University Medical Center Mainz \\ muellka@uni-mainz.de
}

Background: Internet-related disorders (IRD), a poorly controlled engagement in specific internet activities, have negative impact on mental health and psychosocial functioning. Affective and anxiety disorders are frequent comorbid disorder in IRD, yet their effect on the treatment response has not yet been investigated. The objective of the talk is to investigate moderating effects of comorbid disorders on the treatment outcome and drop-out rates.

Methods: The randomized controlled trial STICA was run to evaluate the effects of a cognitive-behavioral treatment program on IRD. Patients $(n=144)$ meeting diagnostic criteria for IRD were included, randomized and allocated to an intervention- group or wait-listcontrol. Primary (diagnostic criteria of IRD) and secondary endpoints (Global Assessment of Functioning; BDI-II; GAD-7) were assessed in 4 measure points.

Results: Comorbid depression was present in $28 \%$ of the patients, anxiety disorders in $18 \%$. Anxiety disorders significantly heightened the drop-out probability $(\operatorname{Exp}(\mathrm{B})=1.77)$ while depression had a negative effect on the IRD-remission rates $(\beta=-.45)$. Both were related to higher IRD-symptoms in the 6-month follow-up $(\mathrm{p}<.05)$. Depressive disorders had an additional deteriorating effect on psychosocial functioning $(\mathrm{p}<.05)$.

Conclusions: Comorbid disorders had a small to moderate influence on the treatment outcome in IRD, stressing the importance of additional therapeutic aspects in this clinical subgroup. 
SY33-4

\title{
Characteristics of Treatment Seeking Problem Gamblers with Adult ADHD
}

\author{
Takahiro Tokumasu ${ }^{1 *}$, Rory C. Reid ${ }^{2}$, Timothy W. Fong ${ }^{2}$ \\ ${ }^{1}$ Showa University \\ ${ }^{2}$ University of California Los Angeles \\ t_tokumasu@hotmail.com
}

The presentation reports characteristics of treatment seeking problem gamblers with adult ADHD ( $\mathrm{n}=39)$ and those without ADHD $(\mathrm{n}=87)$. Patients completed self-report questionnaires about gambling behaviors, impulsivity (UPPS-P), substance abuse (AUDIT / DAST), emotional dysregulation (PHQ-4), illegal activities, and gambling consequences. Each patient received a structured diagnostic interview (MINI) to assess for psychopathology, adult ADHD (ACDS), and gambling disorder (NODS). Results revealed that problem gamblers with adult ADHD encounter unique challenges above those common among problem gamblers including more problems with alcohol / drug abuse and higher levels of impulsivity. Earlier age for first gambling activities, onset of gambling problems, and higher severity of gambling problems was found among gamblers with ADHD. Gamblers with ADHD pawned more items to obtain money for gambling, were more likely to have debt, had significantly higher rates of bankruptcy and domestic violence arising from conflict with family members about their gambling. Interestingly, groups did not differ significantly on their time spent gambling prior to treatment, their win-to-loss ratio, and a number of other gambling-related consequences. These findings are discussed with their clinical ramifications for treatment.

\section{SY34-1}

\section{The relationship between IGD and ADHD: a review}

\author{
Pravin Dullur ${ }^{*}$, Vladan Starcevic ${ }^{2}$, Le Hu Kim ${ }^{3}$ Cam Adair ${ }^{4}$, Daniel King ${ }^{5}$ \\ ${ }^{1}$ Conjoint Senior Lecturer, Western Sydney University, Child and Adolescent Psychiatrist \\ Southwestern Sydney Lhd \\ ${ }^{2}$ University of Sydney \\ ${ }^{3}$ Women's and Children's Health Network South Australia \\ ${ }^{4}$ Game Quitters \\ ${ }^{5}$ University of Adelaide \\ pravin.dullur@health.nsw.gov.au
}

The speakers will discuss the implications of the influence of game design and IGD, identify variables predictive of short-term commitment to gaming abstinence while a one-week follow up survey assessed adherence with intended gaming abstinence. The speakers will then explore the overlap between IGD and other disorders such as ADHD and social anxiety and discuss issues in management 
SY34-2

Problematic online gaming and social anxiety: Towards a better understanding of a complex relationship

\author{
Vladan Starcevic* \\ University of Sydney \\ vladan.starcevic@sydney.edu.au
}

Objectives: This presentation aims to review the relationship between problematic online gaming (POG) and social anxiety. Findings: A positive correlation between POG and social anxiety has been reported by some, but not all studies. This has been interpreted to suggest that individuals with higher levels of social anxiety may be more likely to engage in online gaming, especially when video games are played anonymously and do not require self-disclosure. However, it is unclear how gaming in the context of heightened social anxiety becomes excessive or problematic. Also, social anxiety may become more severe whilst people play video games, suggesting that gaming is not necessarily a self-regulatory activity for socially anxious individuals. It is unclear how a predominance of online over offline relationships in gamers with social phobia affects their levels of social anxiety.

Future research should use a prospective design to investigate any causal relationships between social anxiety and POG. Such research should not consider gaming as a unitary activity because games differ in terms of the extent to which they require certain interpersonal skills and degree to which they provide playing conditions that are perceived as "safe" or otherwise appealing by socially anxious individuals.

\title{
SY34-3
}

\section{"Game Design: Beyond the Loot Box, why online games are the leaders of persuasive design"}

\author{
Huи Kim Le K $^{*}$ \\ Women's and Children's Health Network \\ cgiclinic@gmail.com
}

This presentation by Australian child and adolescent psychiatrist, explores the various methods used by game designers to make online games rewarding for many and addicting for some. The founder of Atari Nolan Bushell once said, "all the best games are easy to learn and difficult to master. They should reward the first quarter and the hundredth." As clinicians treating online gaming addiction, it is important to educate patients about the way games are designed to cater psychologically for the individual player. Clinicians and players alike must be aware of how online gaming companies use persuasive design such as Loot Boxes (synonymous with Havard psychologist B.F Skinner's variable ratio schedule or the "Skinner Box") to make cheap entertainment, which can come at a cost for some players. 
SY34-4

\title{
Clinical predictors of gaming abstinence in help-seeking adult problematic gamers
}

\author{
Cam Adair* \\ Game Quitters \\ cam@gamequitters.com
}

Research into the effectiveness of interventions for problematic gaming has been limited by a lack of data concerning the clinical characteristics of voluntary treatment-seekers; the nature and history of their gaming problems; and, their reasons for seeking help. The study aimed to identify variables predictive of short-term commitment to gaming abstinence following initial voluntary contact with an online help service - Game Quitters. A one-week follow up survey assessed adherence with intended gaming abstinence.

\section{SY35-1}

\section{Criminal defense of Cretmania and its prospects}

\author{
Naomi Sugawarad \\ Daini Tokyo Bar Association \\ sugawara@tamanomori.com
}

What kind of activities are attorneys doing about the incident of Kreptomania? I will report a specific case. In addition to introducing groundbreaking legal cases that have been issued in recent years, we will introduce the limitations and prospects of our efforts.

\section{SY35-2}

\section{Treatment program in prison for pathological gamblers}

\section{Katsuhiro Tamura*}

Niigata prison

tamukatsu900@ybb.ne.jp

Problems of addiction such as alcohol, drugs, gambling affect the crimes of imprisoned inmates. Treatment programs on drugs and alcohol have been implemented in earnest in Japanese prisons, showing effectiveness to prevent repeat offenses. At Niigata prison, a treatment program focused on pathological gambling has begun to be implemented and efforts to prevent repeat offenses are underway. This program is voluntary and has 4 group therapy sessions (60 minutes) and 1 special lecture per month. The session 1 uses the gambling dependency check tool, look back on past lives and accept that the prisoners are pathological gambler. The 
session 2 compares the advantages and disadvantage of stopping gambling. The session 3 helps them to understand the gambling behavior as results of positive and negative reinforcements. The session 4 also helps them to explore their new way of life with debt problems. The finale special lecture is conducted by a peer supporter who actually recovered from pathological gambling. The program aims to be able to find ways to seek out new ways of life so that imprisoned inmates will not return to gambling lives after they are released from prison.

\title{
SY35-4
}

\section{Prevalence of pathological gamblers and pathological internet users in a Japanese prison. Complete survey}

\author{
Kenji Yokotani* \\ Niigata Seiryo University, Graduate School of Clinical Psychology \\ yokotani@n-seiryo.ac.jp
}

Behavioral addiction problems, such as gambling and internet using, were reportedly more frequent in prisoners than in community samples. Although the high prevalence of addiction problems among prisoners was confirmed in Western prisoners, the prevalence of addiction problem was rarely reported in Asian prisoners. To clarify the prevalence of behavioral addiction in Asian prisoners, we conducted complete survey in a Japanese local prison. The participants were 426 prisoners in the prison. They answered the South Oaks Gambling Screen and the Young Diagnostic Questionnaire for Internet Addiction. Results showed that the pathological gamblers were more frequent in prisoners than in community samples. Pachinko was frequently used as gambling. Further, their pathological gambling was also related with their number of imprisonments. On the other hand, pathological internet users were less in prisoners than in community samples. Most of the prisoners had never used the internet before. Treatment of pathological gambling in Japanese prisons and restriction on gambling behavior in Japanese community could be important to reduce the prisoner's risks of reoffending.

\section{SY36-1}

\section{The current situation of psychiatric education system including addiction medicine in Japan.}

\author{
Tomohiro Shirasaka ${ }^{1 *}$, Miyuki Tsuneta ${ }^{1}$, Hisakazu Kimura ${ }^{1}$, Toshikazu Saito ${ }^{2}$ \\ ${ }^{1}$ Department of Psychiatry, Teine Keijinkai Hospital, Sapporo, Japan \\ ${ }^{2}$ Miki mental clinic, Sapporo, Japan \\ shirasaka.t@gmail.com
}

Problematic Internet use and the other behavioral addictions have been a dramatic increase. The prevalence of adults problematic Internet use was estimated 2.71 milions in 2008. Regarding for the Japanese education system, along with the 2-years internship program, psy- 
chiatric residents must take the Psychiatric Specialist Accreditation Program for at least three years, which was put into practice by the Japanese Society of Psychiatry and Neurology (JSPN) in 2006. At hospitals and under supervisory doctors accredited by JSPN, they must accomplish all the following trainings; general clinical psychiatry, psychopharmacology, psychotherapy, psychiatric emergency, consultation-liaison psychiatry, psychiatric rehabilitation and medical ethics among others.

At the same time, they must use the handbook of specialist by JSPN and check whether they can meet a given criteria piece by piece with their supervisors. In addition to the abovementioned course, they must submit 10 case reports minimum and take a multiple-choice exam and interview.

Only in doing so, they will be granted certification as psychiatric specialists. Moreover, after that, they proceed to subspecialties that they are interested in. In this presentation, I would like to talk about the Japanese perspective of Addiction treatment mental health policy and education system.

\title{
SY36-2
}

\section{Addiction Medicine Training in Taiwan}

\author{
Chia Chun Hung ${ }^{1 *}$, Wei Ling Chen ${ }^{2}$ \\ ${ }^{1}$ Bali Psychiatric Center, Ministry of Health and Welfare \\ ${ }^{2}$ Chiayi Branch, Taichung Veterans General Hospital \\ cocohung0418@gmail.com
}

In Taiwan, it takes four years for psychiatric residency training. The addiction medicine is part of the psychiatric clinical training, including 9 hours addiction basic lecture and 20 cases of addiction patient care. In 2008, Taiwanese Society of Addiction was first established. In 2017, the first version of Regulations of Addiction Psychiatry was made then revised a year later. For addiction subspecialty training, currently basic requirement includes a boarded psychiatrist, at least 5-year clinical experience, and has continuing medical education 90 points. Pencil / paper exam and case report are needed for applying Addiction Psychiatry Certificate. The Addiction Psychiatry Certificate needs to be renewed every 6 years, with continuing medical education 120 points. Nowadays, though there are increasing numbers of addiction psychiatrist, unreasonable payments, stigma of addiction patient, and inadequate psychosocial training set the limitation of addiction medicine in Taiwan. Besides, there is no special requirement of addiction treatment for case manager, nurse, psychologist and social worker. Addiction professionals often get experience by starting clinical service. With the urgent needs of addiction treatment, we need to enhance the training quality of addiction professionals., and further to incorporate divert addiction treatment methods and expand treatment capacity. 
SY36-3

\title{
Training for Addiction Medicine: From basic to advanced training
}

\section{Woraphat Ratta-Apha*}

Faculty of Medicine Siriraj Hospital, Mahidol University

woraphatr@gmail.com

After the Thai government launched a "war on drugs" campaign, the need for facilities for comprehensive treatment and related professionals for drug-addicted persons increased. In Thailand, training systems for addiction medicine can be divided into basic training, advanced training, and specialized training courses. Most of the training courses are basic and advanced level for health professionals who work on this field. Regarding the advanced training, there are several training programs provide the clinical rotation for practicing in this field. Among residency training programs, Residency Training in Family Medicine and Residency Training in Psychiatry provided the clinical rotation for addiction medicine. The training programs need the monitoring and supervision by the board of education of each college.

Recently, the Royal College of Psychiatrists of Thailand launched the Residency Training in Addiction Psychiatry. Training requires four years for experiences in general psychiatry and addiction medicine. The curriculum has just passed the certification by the Medical Council of Thailand. Furthermore, in other professions, e.g., nursing, also has some advanced training programs for addiction specialized nursing by the joint programs of addiction institutes and Faculty of Nursing of Universities around the country. All training programs improve the quality of health professionals for facing the problems of addiction that are still challenging.

\section{SY36-4}

\section{The actual situation of psychological experts education programs and treatment systems of illegal drugs and other addiction in Japan.}

\author{
Moritoshi Kido ${ }^{1 *}$, Tatsuya Noda ${ }^{2}$, Nobuaki Takahashi ${ }^{3}$ \\ ${ }^{1}$ Osaka University of Commerce \\ ${ }^{2}$ Nara Medical University \\ ${ }^{3}$ Bukkyo University \\ kido79@daishodai.ac.jp
}

In Japan, according to Ministry of Health, Labor and Welfare, the lifetime experience rate of illegal drugs went up to $2.3 \%$. This percentage is very low in comparison with the U.S. and European countries. In this situation, the total number of arrested by using illegal drug went up to about 14,000 recently. And the number of consultations about drug addiction at the public health centers went up to about 11,000 cases, but it tends to be decreasing. In 
Japan, drug addiction is regarded as criminal not mental disorder, so the illegal drugs are strictly controlled by law and police. But the total number of people who are arrested has not changed and more than half of the total number consists of second conviction. So making the law stricter is less effective for illegal drug use, and the necessity of effective treatment system and method is increasing. In this presentation we introduced the actual situation of the Japanese government countermeasures for illegal drugs and other addiction. Then we introduced the psychological expert's training and education programs for treatment of illegal drugs and other addiction. And we discuss about the future prospects of countermeasure for addictive disorder, especially about the training and education programs.

\title{
SY36-5
}

\section{Training and capacity building for expanding harm reduction services in Malaysia}

\author{
Anne Yee ${ }^{1 *}$, Ahsan Ahmad ${ }^{2}$, Rusdi Bin Abd Rashid', Adeeba Kamarulzaman ${ }^{1}$ \\ ${ }^{1}$ University Malaya \\ ${ }^{2}$ Yale University, School of Medicine \\ annyee17@um.edu.my
}

Drug addiction is a major legal, social and health issue in Malaysia. In 2005, Malaysia's harm reduction program - the methadone maintenance treatment (MMT) program and needle syringe program (NSP) were introduced in response to the escalation of the HIV epidemic. Although the Malaysian government had envisioned to create a drug addiction free nation by the year 2015, the number of drug users in Malaysia is still on the rise. Up to date, more than 2000 doctors are trained but only about $10 \%$ are actively treating drug use patients in their respective settings. This is mainly because they have limited practical knowledge and training on treating drug users with co-morbidities and therefore have no confidence in meeting complex patient needs without the help of professionals. To achieve this, the scale up of harm reduction services, including linkage to care, and treatment, will require moving beyond specialty settings and will necessitate the greater involvement of a broad range of health professionals, especially primary-care providers. Therefore, evidence-based training strategies i.e. the development of a certification program and project $\mathrm{ECHO}$, will need to be integrated to educate urban and rural health care professionals. Through project ECHO, the upscale of harm reduction services, linkage and treatment, is made possible by offering primary-care clinicians continuous training, advise and support in delivering best-practice care for harm reduction. 


\title{
SY37-1
}

\section{Development of the Indonesian Internet Addiction Questionnaire: The new internet addiction screening tool catered for Asians}

\author{
Kristiana Siste Kurniasanti ${ }^{1 *}$, Hans Christian ${ }^{2}$, Karina Kalani Firdaus $^{2}$, Tjhin Wiguna ${ }^{1}$, \\ Raden Irawati Ismail ${ }^{1}$, Martina Nasrun Wiwiel \\ ${ }^{1}$ Department of Psychiatry, Universitas Indonesia-Cipto Mangunkusumo National Hospital \\ ${ }^{2}$ Faculty of Medicine, Universitas Indonesia-Cipto Mangunkusumo National Hospital \\ ksiste@yahoo.com
}

With the increase usage of internet in Indonesia, so does the vulnerability of adolescents to be addicted to internet. In a preliminary research conducted in Indonesia, the internet addiction prevalence reached $20 \%$, but unvalidated instruments were used in the study. Adolescence is a critical phase in the development of an individual hence the need of early detection for problems, including internet addiction, leading to appropriate immediate intervention. Early detection of internet addiction in adolescents requires a validated questionnaire compatible with the Indonesian adolescent culture, in which until this research has been absent.

Since 1996, the detection of internet addiction with the Internet Addiction Test, a validated questionnaire with good reliability used worldwide. Among the limitations of the test are the absence of culture aspect and language especially in non-english speaking countries. In the latest revision, countries all over the world are asked and pushed to develop their own questionnaire adjusted to their local language and culture that can also be used as benchmarking for other countries. Our research was aimed to develop a screening tool, the Indonesian Internet Addiction Questionnaire, composed of 14 questions, catered for young Adolescents in Indonesia, and validated with good reliability (cronbach alpha 0,881).

\section{SY37-2}

\section{Imaging studies of gambling disorder in Japan}

\author{
Kosuke Tsurumi* \\ Kyoto University Graduate School of Medicine \\ kaytee@kuhp.kyoto-u.ac.jp
}

Gambling disorder (GD) is a chronic mental disorder which affects the well-being of patients as well as that of people around them. Although GD could bring severe negative consequences, countermeasures to tackle GD is insufficient. This might be partly because of the lack of effective pharmacotherapy. Investigating the neural basis of GD could improve this situation. We focused on the insular cortex and found decreased activation during reward anticipation in GD patients. We are now investigating insular resting state functional connectivity.

Considering the close relationship between GD patients and money, we have applied some behavioral economics tasks. Among them, using loss aversion parameter, we successfully 
classified GD patients into 2 subgroups and found common and differential structural abnormalities. These results might lead to tailored pharmacotherapy according to individual risk preference in GD patients. We also found an association between regional brain volume and risky probability cognition in GD patients.

Apart from the typical behavioral economic paradigms, we investigated the neural activation during state-dependent risky decision making. We also investigated the neural basis of 'chasing', the specific condition in GD patients.

These results would shed light on the pathophysiology of GD, which could potentially give a clue to future treatment.

\title{
SY37-3
}

\section{Factor structure of the Game Addiction Screening Test (GAST) in Thai adolescents}

\author{
Sawitri Assanangkornchai*, Suwanna Arunpongpaisal, Darika Saingam \\ Prince of Songkla University \\ savitree.a@psu.ac.th
}

Background: The Game Addiction Screening Test (GAST) is a 16-item screening instrument for problematic gaming in children and adolescents. It has two versions, child / adolescent and parent versions. This study aims to explore the construct validity of the GAST and determine factors associated with problematic gaming in Thai high-school students.

Methods: Data of 38,186 high-school students of the 2016 national school survey was analysed, using exploratory and confirmatory factor analyses (EFA and CFA) and multivariate logistic regression.

Results: The EFA revealed three domains, which were supported by the CFA; salience, dysfunction and mood change. Of all $7.7 \%$ and $2.3 \%$ were screened positives for gaming problem and gaming addiction, respectively by the GAST. Factors significantly associated with problematic gaming were gender, age, region, academic performance and school type. Students with problematic gaming were more likely to spend more time and money on gaming, reduce sleeping time and physical activity, use alcohol / tobacco / drug and have depression, suicidality and fighting than were non-problematic gaming students. Conclusion: The GAST appears to be a satisfactory tool to screen for problematic gaming in Thai adolescents. 
SY37-4

\title{
Psychometric development of the Problematic Stock Trading Scale
}

\author{
Sungwon Roh'* , Tae Kyung Lee ${ }^{2}$, Joohyun Han ${ }^{3}$, Euihyeon $\mathrm{Na}^{4}$, Minkyung Kang ${ }^{1}$ \\ ${ }^{1}$ Hanyang University \\ ${ }^{2}$ Chuncheon National Hospital \\ ${ }^{3}$ National Center for Mental Health \\ ${ }^{4}$ Incheon Chamsarang Hospital \\ swroh@hanyang.ac.kr
}

Despite the increased number of retail investors and growing concern over their addictive behavior over the past few decades, there are no reliable and valid instruments for assessing problematic stock trading. The aim of this study is to develop the Problematic Stock Trading Scale (PSTS), a valid and reliable scale that measures problematic stock trading.

A sample of 500 retail investors was used in this study. Twenty-two items (nearly twice the final number of items) were initially selected as preliminary items, based on previous addiction scales such as gambling and substance use disorders as well as the clinical experience of the experts involved. The final items on the PSTS were determined through an exploratory factor analysis.

Three core factors related to problematic stock trading were revealed from the exploratory factor analysis. The 12-item PSTS consists of five 'Preoccupation' factor items, four 'Risky Investment' factor items, and three 'Cognitive Distortion' factor items. Reliability and external validity were confirmed.

The present findings highlight the potential use of the PSTS for future research and possibly for clinical application by defining problematic stock trading as a behavioral addiction.

\section{SY37-5}

\section{Management of gaming disorder and internet addiction in Japan}

\author{
Satoko Mihara*, Hideki Nakayama, Susumu Higuchi \\ National Hospital Organization Kurihama Medical and Addiction Center \\ mihara.satoko.pd@mail.hosp.go.jp
}

Background: The treatment of gaming disorder (GD) and internet addiction (IA) is in its infancy worldwide. In an attempt to gauge the treatment and counseling capacity in relation to GD and IA in Japan, we conducted surveys of mental health and welfare centers (MHWC) in 2016 and 2018. These centers are the principal facilities providing consultation services for mental health issues in pre-fectures and major cities.

Methods: The survey aimed to clarify the centers' functionality in the early identification of GD and IA. It also included questions to identify those medical and non-medical facilities 
providing specialist treatment or counselling services. In addition, the number of patients treated and treatment mo-dalities conducted in these facilities were also surveyed.

Results: The number of people who sought consultation in relation to GD / IA at MHWC increased 5-fold between 2013 and 2017. The number of outpatients with GD / IA who visited specialist treatment facilities increased by a factor of three during the same period, while the number of these facili-ties increased 2-fold between 2016 and 2018.

Conclusion: Our study suggests that the number of treatment and consultation seekers with GD / IA in Japan is increasing at a rapid pace that the treatment and consultation capacity is struggling to match.

\title{
SY38-1
}

\section{Smartphone use and behavioral problems in young children: Evidence from South Korea}

\author{
Yunmi Shin ${ }^{1 *}$, Sungju Kim², Hee Jeong Yoo ${ }^{3}$, Eunjun Park ${ }^{4}$ \\ ${ }^{1}$ Ajou university, School of Medicine, Department of Psychiatry. Suwon, Republic of Korea \\ ${ }^{2}$ Ajou university \\ ${ }^{3}$ Bundang Seoul university \\ ${ }^{4}$ Ilsan baik hospital university \\ ymshin@ajou.ac.kr
}

Drawing on a longitudinal sample of young children in South Korea where smartphone penetration rate is among the highest, we aim to investigate the association between the use of smartphone and current and prospective behavior problems among young children (2-5 years old). We analyze 367 children at the age of 2 to 5 years based on the first and second waves of the I-CURE (Internet-Cohort for Understanding of internet addiction Risk factors / Rescue in Early livelihood) study where 400 caregivers of 2- to 5-year-old children were invited to enroll. We regress mother-reported behavior problems scores assessed with K-CBCL on mother-reported time for media-use of their children. Smartphone use more than one-hour per day is related to more internalizing and externalizing behaviors among young children, and also to sleep disorder, attention deficits, and aggressive symptoms. Television use was irrelevant to child problem behaviors except for attention deficits. Early smartphone use may lead to internalizing and externalizing behaviors among young children, especially to sleep disorder, attention deficits, and aggressive symptoms. These results imply that smartphone use among young children should be carefully monitored and controlled by mothers. 


\title{
SY38-2
}

\section{Prospective association between Internet gaming behaviors and Internet gaming disorder: 24-month follow-up results from the iCURE study}

\author{
Hyeon Woo Yim ${ }^{1 *}$, Sun-Jin Jo ${ }^{1}$, Hyunsuk Jeong ${ }^{1}$, Hye Jung Son ${ }^{1}$, Ji-Yoon Choi ${ }^{1}$, \\ Hae-Kook Lee ${ }^{2}$ \\ ${ }^{1}$ The Catholic University of Korea \\ ${ }^{2}$ The Catholic University Uijungbu St. Marys Hopital \\ jiny4u@catholic.ac.kr
}

This study was carried out to investigate the effects of Internet gaming behaviors on occurrence of Internet gaming disorder. For this purpose, data from a child and adolescent prospective cohort study, the Internet user Cohort for Unbiased Recognition of gaming disorder in Early adolescents (iCURE) were analyzed. Among 2,319 participants enrolled at baseline, 1,920 middle school students were included in this study, and data of 1,776 follow-up at 24 months were analyzed.

Game use behavior, which is an exposure variable, was based on baseline data, including time and location of game use. The outcome variable, Internet gaming disorder (IGD), was measured using the Internet Game Use Elicited-Symptom Screen (IGUESS), a self-reported reliable and valid measure based on DSM-5 diagnostic criteria for IGD. At the 24-month follow-up, the IGUESS total score of 10 points or more was defined to be positive for internet gaming disorder risk.

Based on the results of multiple logistic regression analysis, we examined the effect of game use behavior on the occurrence of IGD risk, and the implications of the findings were discussed from a public health perspective.

\section{SY38-3}

\section{Changes in the prevalence of internet addiction among adolescent and adult populations in Japan}

\author{
Aya Kinjo ${ }^{1 *}$, Yoneatsu Osaki ${ }^{1}$,Satoko Mihara ${ }^{2}$,Hideki Nakayama ${ }^{2}$,Susumu Higuchi ${ }^{2}$ \\ ${ }^{1}$ Tottori University \\ ${ }^{2}$ Kurihama Medical and Addiction Center \\ kinjo_aya@tottori-u.ac.jp
}

The nation-wide school-based and population-based surveys for internet addiction (IA) were conducted in 2012-2013 and 2017-2018 in Japan. Individuals who got five points or more on Young's diagnostic questionnaire (YDQ) were defined as having IA. The prevalence of IA was $12.4 \%$ among junior high school students, $16 \%$ in senior high school students and $1.1 \%$ among adults in the 2017-2018 survey in Japan. The prevalence doubled among students 
and tripled among adults compared to the 2012-2013 survey. The most popular devices used for internet connection have changed from the personal computer and gaming console in 2012-2013 to the smartphone in 2017-2018.

In the school-based survey, the percentages of IA were significantly higher among students who attended high school, were girls, smokers and drinkers in both surveys. The percentage increase of IA was more rapid in lower grade students. The population-based survey showed that IA has spread among those who were 60 years old and older in 2018, while the percentage of IA was higher in the younger age group in both survey years. The control policy to prevent IA should be constructed.

\title{
SY38-4
}

\section{Protective Factors against Internet Gaming Disorder: A Longitudinal Study}

\author{
Anise M.S. Wu*, Meng Xuan Zhang, Xinrui Wang, Shu Yu \\ University of Macau \\ yb77304@um.edu.mo
}

Background: While psychosocial risk factors of Internet gaming disorder (IGD) were well-documented, there is very limited research, particularly with longitudinal design, on protective factors against IGD. Based on previous findings, this study identified purpose in life and social support as potential protective factors and tested if they actually lowered ones' IGD symptoms over time among youths.

Method: Two hundred and eighty-three university students, recruited at a public university in Macao, China, participated in an anonymous questionnaire survey in 2016 (W1) and a oneyear follow-up study (W2).

Results: As hypothesized, social support and purpose in life had significantly negative correlations with IGD in both waves ( $\mathrm{ps}<.05)$. Results of cross-lagged panel analysis showed that higher purpose in life at W1 $(\mathrm{p}<.05)$, but not social support, significantly predicted the lower level of IGD at W2. The finalized cross-lagged model also supported a bidirectional association between purpose in life and social support (ps $<.05)$.

Conclusions: Our findings showed that purpose in life was a salient protective factor against IGD, while social support and purpose in life had a reciprocal relationship. Strategies to cultivate and search for purpose / meaning in life should be considered in IGD intervention for Chinese youths. 
SY39-1

\title{
How parents sometimes deliberately increase screen time of their child
}

\author{
I. M. Koning*, R. de Bok, S. Geurts, R. J.J.M. van den Eijnden \\ Utrecht University, The Netherlands \\ i.koning@uu.nl
}

Previous research has demonstrated that the influence of commonly studied parenting practices (e.g. rules about internet use) on screentime behavior of youth is trivial. In the current study, a new aspect of parenting in relation to children's screentime behavior is investigated, i.e. parents' self-interest. In contrast with several other risk behaviors, such as delinquency, we expect that parents do not intervene or even encourage the use of screens among offspring because of their own interest, me-time. In this qualitative study we held interviews with 30 parents of 6-16-year-old children about potential situations wherein they indicated to have a self-interest in their children's online behavior and how this me-time is obtained (encourage, not intervene, allow). The results of this study will be presented in light of the broader role of parents in children's online behavior.

\section{SY39-2}

\section{The impact of social media disorder symptoms and social media use on sleep, and the preventing role of parental rules}

\author{
R. J.J.M. van den Eijnden*, S. Geurts, I. M. Koning \\ Utrecht University, The Netherlands \\ R.J.J.M.vandenEijnden@uu.nl
}

\begin{abstract}
A wide range of studies has linked sleep problems with screen-based activities in general (see for review Cain \& Gradisar, 2010). However, research on sleep and social media use specifically is limited. Therefore, we examined the longitudinal effects of frequency of social media use as well as symptoms of social media disorder (SMD) on adolescents' bedtime and perceived quality of sleep. Furthermore, we tested whether these effects were moderated by parental rules about using the Internet an hour before bedtime. As part of the Digital Youth Project of the University of Utrecht, a two-wave longitudinal sample of 13-17-year-old adolescents $(\mathrm{N}=1.422)$ was utilized. The results indicated that quality of sleep was negatively affected by symptoms of social media disorder, and bedtime was negatively affected by frequency of social media use. Interestingly enough, strict parental rules about using the internet one hour before bedtime only seemed to prevent sleep problems for moderate users of social media with low symptoms of social media disorder, but not for highly-engaged social media users and users with more social media disorder symptoms. The results of this study will be discussed in the light of future preventive interventions aiming at adolescents and their parents.
\end{abstract}


SY39-3

\title{
Social networking addiction among university students in Hong Kong
}

Lu $\mathrm{Yu}^{*}$

Department of Applied Social Sciences, The Hong Kong Polytechnic University

lu.yu@polyu.edu.hk

The present study estimated the prevalence rate of Social Networking Addiction (SNA), an emerging public health issue, based on a sample of 390 Hong Kong university students (age $=19.09 \pm 1.47$ years), and examined its association with multiple indicators of well-being including life satisfaction, depression, academic achievement, and sleeping quality. The relationship between Internet specific parenting behaviors and university students' SNA was further investigated. Results showed that about $20 \%$ of the participants met the criterion of SNA measured by the Social Media Disorder Scale. Students with SNA reported lower life satisfaction, more depressive symptoms, lower self-perceived academic performance, and poorer sleeping quality, as compared to students without SNA. Parents of students with SNA showed not only more limits and intervention on their children's online behaviors, but also more co-use and their own use of social media. Longitudinal studies based on a more representative sample are critically needed in the future.

\section{SY39-4}

\section{Social media disorder, ADHD-symptoms, and mental health: Longitudinal investigations of directionality among adolescents}

\author{
M. Boer", G. W.J.M. Stevens, C. Finkenauer, R. J.J.M. van den Eijnden \\ Utrecht University, The Netherlands \\ m.boer2@uu.nl
}

Cross-sectional research shows that symptoms of social media disorder (SMD) and symptoms of Attention Deficit Hyperactivity Disorder (ADHD) are related. Due to the lack of longitudinal studies, the direction of this relation remains unknown. The present study aims to address this gap by investigating the bidirectional relation between ADHD-symptoms and SMD- symptoms using longitudinal data. Also, the direction of the relation between ADHDsymptoms and frequency of social media use was examined. A three-wave longitudinal study among Dutch secondary school students aged $11-15$ years was used $(n=543)$. Findings from a random intercept cross-lagged panel model suggest a unidirectional relation, whereby SMD- symptoms increased ADHD-symptoms over time. Furthermore, no longitudinal associations between frequency of social media use and ADHD-symptoms were found. This implies that disordered use of social media, rather than highly-engaged use of social media, has harmful implications for adolescents' ADHD-symptoms. In addition, bidirectional re- 
lations between SMD-symptoms and mental health outcomes will be examined, including life satisfaction and depression symptoms. Findings of these studies are considered to be an important step towards understanding the relation between disordered use of social media and adolescent mental health. Promising directions for continuing longitudinal research will be discussed.

\title{
SY40-1
}

\section{Diminished Goal-directed Cognitive Control in Gaming Disorders}

\author{
Young-Chul Jung*
}

Yonsei University, College of Medicine

eugenejung@yuhs.ac

Gaming disorder (GD) is characterized by impaired control over gaming, and continuation of gaming despite the occurrence of negative consequences. We hypothesized that individuals with GD would show weaker goal-directed top-down attention, while playing games that require real-time strategies, compared to healthy controls (HC).

We measured the grey matter volume and resting state MR functional connective in 33 individuals with GD and $29 \mathrm{HCs}$ (mean age $=22.0 \pm 2.8$ years). Then the cortical electrical activity and autonomic nervous system activity were analyzed, while the participants played their favorite multiplayer online battle game. Each participant played three consecutive games, which took 30 40 minutes per game.

The GD group showed weaker frontal theta and alpha activity (electroencephalography) and weaker high-frequency bands (heart rate variability), which indicated weaker cognitive control while playing games compared to the HC group. In addition, the weaker high frequency HRV correlated with weaker dorsal dorsolateral prefrontal cortex-inferior frontal gyrus connectivity $(\mathrm{P}=0.018)$ and stronger dorsal caudal putamen-postcentral gyrus connectivity $(\mathrm{P}=0.036)$.

Our findings suggest an imbalance between the stimulus-driven attention network and the goal-directed top-down attention network in individuals with GD. These alterations should contribute to the diminished cognitive control over gaming, resulting in stimulus-driven compulsive gaming behaviors. 
SY40-2

\title{
Radixin in the nucleus accumbens contributes to decision-making toward risk choice
}

\author{
Jeong-Hoon Kim*
}

Yonsei University, College of Medicine

jkim1@yuhs.ac

The ezrin-radixin-moesin (ERM) proteins have been implicated both in cell-shape determination and in cellular signaling pathway. It was previously shown that psychomotor stimulants decrease phosphorylation levels of ERM proteins in the nucleus accumbens (NAcc), an important brain area mediating addictive behaviors. However, it has not been determined yet what functional role ERM proteins play in this site in relation with decision-making toward risk choice. In this presentation, it will be shown how radixin, one of the ERM proteins abundantly expressed in the NAcc, affects the preference of choice in rat gambling task (rGT), an animal model which shares many features of the human gambling tasks including uncertainty, reward and punishment. Once animals showed a stabilized pattern of preference in rGT, they were bilaterally microinjected with lenti viral vectors, which contain either GFP alone, wild-type or mutant radixin gene, into the NAcc core. Interestingly, overexpression of wild-type radixin in the NAcc changed the choice preference toward averse in a previously categorized seeking group. These results first time indicated that radixin in the NAcc core plays a role in controlling the preference of risk choice, providing a clue to molecular basis for decision-making toward risk choice behavior.

\section{SY40-3}

\section{Functional neural changes after dropping-off addictive behaviors for Internet gaming disorder: Implications for its negative effect}

\author{
Guangheng Dong ${ }^{1 *}$, Min Wang ${ }^{2}$, Jialin Zhang ${ }^{2}$, Xiaoxia Zhou ${ }^{3}$ \\ ${ }^{1}$ Hangzhou Normal University \\ ${ }^{2}$ Zhejiang Normal University \\ ${ }^{3}$ East China Normal University \\ dongguangheng@zjnu.edu.cn
}

Backgrounds: Although studies have indicated that Internet gaming disorder (IGD) could impair cognitive functions of players, however, most of these results are less conclusive as they used between-group comparison study designs.

Methods: We scanned a group of IGD subjects (154) when they are in addiction and rescanned those who stopped their addictive behaviors (29) after one year when they were performing the cue-craving tasks. We compared their brain features when facing gaming cues between in IGD and after dropping off. 
Results: Subjects' cravings to gaming cues decreased significantly after dropping-off their addictive behaviors. Decreased brain responses in anterior cingulate cortex (ACC) and lentiform were observed in dropping-off situation. Significant positive correlation was observed between the changes in brain activities in lentiform and changes in self-reported cravings. Dynamic causal model analysis showed increased ACC-to-lentiform connectivity when dropping off their addictive behaviors.

Conclusions: After dropping-off their IGD, people are less sensitive to gaming cues and their executive control ability becomes more efficient in controlling their cravings. We can backward infer that IGD could bring negative effect to players, including increase their sensitive to gaming cues and disturb their executive control. 


\title{
INDEPENDENT ORAL PRESENTATION
}

IO1-1

\section{Internet-addiction prevention: A revised content-based approach to evaluation and prevention}

\author{
Moshe Israelashvili* \\ School of Education, Tel Aviv University \\ mosheil1@post.tau.ac.il
}

An accumulating number of recent studies (e.g., Israelashvili, Kim \& Bukobza, 2012) suggests that sometimes internet over- use - or Compulsive Internet Use (CIU) - represents a positive effort in pursuing of one's life goals and challenges rather than a compulsive (Internet) Use (i.e., CIU). Accordingly, the current study hypothesized that for some people and / or in certain circumstances the internet and related tools (e.g., social media) actually supplies a new and improved way of dealing with daily hassles as well as major life encounters. To explore this hypothesis the current study checked how people $(\mathrm{N}=2,177)$ use the internet, both in general and especially as a way of coping. Data analysis indicated a vast use of the internet by all participants, with no significant age, sex or cultural differences. Using factor analyses and multivariate analyses, in which traditional and internet-related ways of coping were compared, demonstrated that the internet provides at least two new ways of coping with stress encounters, which are currently absent from all existing questionnaire, and discussions on, ways of coping - i.e., (1) exploring normalization, and (2) feeling relatedness. The study findings supply a basis for a better tuned approach to deal with internet-addiction.

\section{IO1-2}

\section{Development of a Manual on Psychotherapy for Addiction to Gaming and Social Networking}

\author{
Rabert Farnam ${ }^{1 *}$, Afarin Rahimi-Movaghar ${ }^{1}$, Kamran Ghani ${ }^{2}$, Hossein Rafiemanesh ${ }^{3}$, \\ Marzieh Hamzehzadeh ${ }^{1}$, Maral Mardaneh Jobehdar ${ }^{1}$ \\ ${ }^{1}$ Iranian National Center for Addiction Studies (INCAS) Tehran University of Medical Sciences \\ ${ }^{2}$ Mardaneh Jobehdar \\ ${ }^{3}$ Beheshti Univ \\ rabertfarnam@gmail.com
}

Introduction: With an increase in the problematic use of games and social networks, the knowledge for the treatment should be enhanced. This paper describes the newly developed manual of PAGS (Psychotherapy for Addiction to Gaming and Social Networking).

Method: This manual was developed at the Iranian National Center for Addiction Studies (INCAS) with financial support from the Iranian ministry of health. The basis for develop- 
ment of the manual was CBT-IA and STICA. The expert group at INCAS made changes according to their clinical experiences. Then, the manual was utilized for treatment of six patients and the manual was revised, accordingly.

Results: PAGS integrates several areas of intervention structured into 3 stages and 8 sections. Stages include: 1) preparation for treatment (2 sections); 2) abstinence and relapse prevention (3 sections); 3) protracted balanced use (3 sections). Sections include: 1) motivational (decision making and acceptance); 2) evaluation and goal setting; 3) relapse prevention (addiction stimulus control and planning for alternative activities); 4) relapse prevention (cognitive restructuration and alternative activities); 5) focus on emotion and addiction (emotional regulation and internal emotional trigger management); 6) work on contextual factors (intrapersonal, self-esteem, self-confidence); 7) work on comorbidities (stress, depression, guilt); 8) protracted addiction management.

\title{
IO1-3
}

\section{Determinants for the effectiveness of an Online Ambulatory Service for Internet Addicts (OASIS)}

\author{
Laura Bottel $^{*}$, Jan Dieris-Hirche ${ }^{1}$, Bert Theodor Te Wildt ${ }^{2}$ \\ ${ }^{1}$ LWL-University Hospital Ruhr-University Bochum, Germany \\ ${ }^{2}$ Psychosomatic Clinic Monastery Diessen, Germany \\ laura.bottel@web.de
}

Background: Low-threshold offerings have been proven to be crucial and effective in reaching out for patients. Because of this the Online Ambulatory Service for Internet Addicts (OASIS) has been developed to offer consultations for adult Internet addicts.

Methods: After a self-assessment in line with the DSM-V criteria for Online Gaming Disorder, individuals at risk are invited to participate in the program. OASIS provides two webcam-based consultation hours, one for thorough clinical diagnostics and one for motivational interviewing and referral for offline treatment. Participants completed questionnaires before and after the consultation hours measuring internet addiction severity (sIAT), motivation for change (iSOCRATES) and daily media use.

Results: Over 120 internet addicts used the online ambulatory service. The results indicate a low-threshold online consultation is a good device to reduce internet addiction severity and increase motivation to change. The positive participants' feedback underlines the satisfaction with the webcam-based consultation hours as well as the simplicity of handling the online tool. Conclusions: Since the results indicate the effectiveness of the online consultation hours, OASIS is meant to be disseminated into the general addiction health system. 


\title{
IO1-4
}

\section{Development of a treatment planning tool for clinical use with individuals who experience problematic video gaming: The Inventory of Antecedents to Problem Gaming}

\author{
Jing Shi ${ }^{1 *}$, Nigel E. Turner ${ }^{1}$, Marc N. Potenza ${ }^{2}$ \\ ${ }^{1}$ Centre for Addiction and Mental Health \\ ${ }^{2}$ Yale University School of Medicine \\ j.shi@mail.utoronto.ca
}

Background: Although the characteristics and measurement of gaming disorder are debated, harms associated with the disorder are widely recognized. Both functional and psychological impairments have been associated with gaming problems. The influx of individuals seeking treatment for gaming problems over the past decade has generated a demand for practical assessment tools that may be used to efficiently target interventions.

Aim: To develop the Inventory of Antecedents to Problem Gaming (IAPG) that assesses clients' triggers to heavy video gaming.

Methods: This mixed-methods study design utilizes quantitative and qualitative approaches. Stakeholders consisting of clients, therapists, caregivers, and recreational gamers will be interviewed in focus groups to develop an initial set of items that will be sorted conceptually into preliminary groups. Online survey data $(\mathrm{N}=300)$ will be used to determine conceptual consistency and if there is a need to reduce the item sets. Further focus group feedback will be obtained to determine face validity.

Conclusions: This research will result in an assessment tool that identifies antecedents to gaming problems that may be used for treatment planning or for management of gaming disorder. The antecedents identified in the instrument may inform prevention strategies that educate youth on the potential harms / dysfunctions associated with gaming disorder.

\section{IO1-5}

\section{Clinical use of the I-PACE-model in treating severe Internet related disorders in adolescents- possibilities and restrictions}

\author{
Oliver Bilke-Hentsch*, Daniel Baumann \\ Modellstation SOMOSA Winterthur \\ oliver.bilke-hentsch@somosa.ch
}

The residential treatment of severe internet use disorders (IRD) with long term comorbidity includes several different approaches both in forensic and non-forensic contexts.

The I-PACE-model (Brand et al., 2017) can be seen as a promising framework for organising and priorising treatment goals and methods. 
Using the the I-PACE-model as theoretical background we developed a multisystemic modular approach in the residential institution / psychiatric hospital "Modellstation SOMOSA" in Winterthur $(\mathrm{CH})$ for 14-19 y old male patients including 4 modules:

1. Individualised and group psychotherapy (psychodynamic, systemic, CBT); medication

2. SOMOSA Media-Lab (office - applications, social media, etc.), mainly individualised

3. SOMOSA game - lab (serious games), mainly individualised

4. Job-assessment (programming of 2- and 3D-printers; industry 4.0), mainly standardised

The first four years (modules 1. and 2.) and the first year (modules 3. and 4) show a successful implementation in particular for severe cyber-addicts and ASS- and ADHD-patients in terms of longer attention span, better overall motivation for professional goals, deeper understanding of underlying conflicts and even more - real life "offline" - contacts.

Patient ressources and deficits can be targeted individually using the I-PACE model. A gender-oriented specification of the SOMOSA-concept for girls (14-18) and patients in emerging adulthood (18-27) is currently under way.

\section{IO2-1}

\section{Ignoring Methodological Problems when Assessing Gambling Prevalence}

\section{Alfred Uhl}

Austrian Public Health Institut and Sigmund-Freud-Private-University alfred.uhl@uhls.at

The prevalence of pathological gambling in the general population is considered a relevant parameter when prevention and treatment issues are discussed. These estimates generally result from large general population surveys. For practical and economic reasons large surveys cannot rely on specifically trained highly qualified experts, but have to be performed by more or less well-trained professional interviewers. The latter can only achieve their related to low prevalent phenomena satisfactory if they can use highly reliable and valid assessment tools. Since the prevalence of pathological gambling in many countries lies in the one-percent range, meaningful results are only conceivable if the psychometric properties of these instruments are truly excellent. Scientists developing screening instruments, validating them and / or using them commonly claim that the psychometric properties of their instruments are good to excellent - based on empirical results that actually suggest something quite different - and commonly they get away without dissent. The presentation will use simple calculations based on generally accepted formulas, to demonstrate and quantify the problems arising when the sensitivity and / or specificity of the applied tools is suboptimal. Subsequently, the question will be discussed whether the application of common screening tools and questionnaires in general population surveys to assess low prevalent phenomena is justified, if instruments are far from perfect. 


\title{
IO2-2
}

\section{The Near-Miss Effect in Slot Machines: An Exploration of Virtual Reality and Laboratory Gambling Environments}

\author{
Steve Sharman ${ }^{1 *}$, Amanda Roberts ${ }^{2}$, Volker Thoma ${ }^{1}$, John Turner ${ }^{1}$ \\ ${ }^{1}$ University of East London \\ ${ }^{2}$ University of Lincoln \\ ssharman@uel.ac.uk
}

The near-miss effect is a key construct in disordered gambling. A near-miss occurs when the gambler feels that they were close to winning, even though the objective outcome is still a loss. Much previous research in this area has used either real slot machines in a laboratory or programmed slot machine simulations creating a controlled experimental environment. Although such methodologies allow retention of experimental control, such studies lack ecological validity and therefore have limited external application. Alternatively, gamblers can be observed in naturalistic environments such as casinos, however although ecological validity is increased, experimental control is somewhat relinquished.

This research is the first in the UK to investigate gambling behaviour fully immersive using virtual reality (VR) technology. VR allows us to harness the ecological validity afforded by naturalistic studies, whilst retaining full experimental control. Participants completed a slot machine simulation in one of three different environments: i) delivered on a PC in a psychology testing room, ii) in computer generated fully immersive casino, and iii) via fully immersive real world 360-degree footage. Results compare participant sense of presence, and gambling persistence across experimental conditions. The results, and implications for experimental psychology research in gambling behaviour are discussed.

\section{IO2-3}

\section{Community-based approach for Behavior Addiction}

\author{
Munenori Katayama*, Kaori Aizawa, Sachiko Nagata, Norihito Shirakawa \\ Mental Health and Welfare Center, City of Yokohama \\ k2535027@kadai.jp
}

Recent studies show that cases of behavior addiction are increasing in local governmental facilities in Japan. Since these facilities are public agencies, they are capable of treating the patients not just inside their facilities, but by utilizing the local network which these local facilities have, such as collaboration of welfare offices, public health care offices, and community workshops. It is assumable that these approaches would be essential for an effective treatment of the disorder, there are limited studies which report the effectiveness of the community-based approach for patients with behavior addiction, especially in Japan. This 
study will present multiple case reports of how community-based approach are currently provided for people with various behavior addiction in the 18 wards and Central Mental Welfare Center of Yokohama city. The aim of the study is to present the possible advantages and shortcomings of the approach, and key features for further research shall be quoted.

\title{
IO2-4
}

\section{Responsible gambling: Associations with its expectancies and gambling disorder}

\author{
Anise M.S. Wu*, Juliet H. Chen, Kwok Kit Tong \\ University of Macau \\ anisewu@um.edu.mo
}

Responsible gambling (RG) approach, which involves multiple stakeholders, has been adopted and promoted by the Macao government to combat gambling-related problems, including gambling disorder. Earlier surveys however, have shown that a fair proportion of gamblers do not engage in personal RG practice (e.g., set limits of gambling expense and time). Based on the health belief model, this cross-sectional study investigated if and which expectancies regarding $R G$ were associated with $R G$ practice and if $R G$ practice were protective factors of gambling disorder. We conducted a telephone poll in Macao, China and randomly recruited 203 Chinese adults (male $=57 \%$; age $=18-81$ years), who had engaged in gambling activities in the past 12 months, for an anonymous survey. We found that RG practice was not significantly correlated with perceived barriers / benefits and self-efficacy regarding RG practice $(\mathrm{p}>.05)$. Gamblers however, reported more RG practice if they perceived more negative consequences of gambling and higher severity of gambling disorder $(p<.01)$. $R G$ practice was also negatively correlated with self-reported DSM-5 symptoms of gambling disorder $(\mathrm{p}<.01)$. Our findings suggest that future RG campaigns should consider segmentation and incorporate some strategies that increase adverse consequences of (disordered) gambling perceived by gamblers.

\section{IO2-5}

\section{Social influences normalise gambling, and gambling-related harm, amongst higher-risk gamblers}

\author{
Alex Myles Thomas Russell*, Erika Langham, Nerilee Hing \\ CQUniversity Australia \\ a.m.russell@cqu.edu.au
}

Social influences are key drivers of many behaviours, including physical exercise, drinking and gambling. For gambling, social influences include early exposure through parental involvement, and over time, friends and colleagues. This study aimed to compare social influ- 
ences across gambling risk groups, by examining key characteristics of their social networks using egocentric social network analysis. A total of 784 Australian adults (egos) reported their demographics, gambling behavior and problem gambling severity, as well as those of the 20 most influential people in their lives (alters). Egos also reported the strength of the connection between themselves and each of their alters, and between each pair of alters. Egos in higher risk groups reported more alters who gamble, and who experience gamblingrelated problems, and closer connections to their alters, whether the alters gamble or not. Furthermore, the networks of higher risk gamblers were more interconnected - more alters know each other. These findings indicate that both gambling behaviour and gambling-related problems are normalised by social connections. The higher degree of interconnectedness in the networks for higher risk egos indicate limitations of individualised interventions, and instead highlight the important role of changing norms within society, which can be transmitted throughout these networks.

\title{
IO3-1
}

\section{Prediction of internet gaming disorder based on integrative analysis of EEG and PET data}

\author{
Donghwan Lee ${ }^{I^{*}}$, Boram Jeong ${ }^{1}$, Ji Yoon Lee ${ }^{2}$,Heejung Kim², Jung-Seok Choi², \\ Youngjo Lee Le $^{3}$ \\ ${ }^{1}$ Ewha Womans University \\ ${ }^{2}$ SMG-SNU Boramae Medical Center \\ ${ }^{3}$ Seoul National University \\ 94ram@naver.com
}

Internet gaming disorder (IGD) becomes an important issue and is one of the most discussed psychosocial problems. To prevent IGD and provide appropriate intervention, an accurate prediction method for identifying IGD is necessary. In many works of literature, it is discovered that brain-related indicators such as Electroencephalography and Positron Emission Tomography, and the other clinical features can be associated with IGD. In this study, we propose an IGD prediction method by integrating all three modalities to enhance prediction accuracy. In such multimodal data, each modality has its own source, and its characteristics are different. Unlike the conventional methods which usually concatenate all features into one feature vector, we adopt a multiple-kernel support vector machine (SVM) to identify the IGD patient. By using real data collected from Korean adults, we compare the prediction performance of standard machine learning methods such as SVM, random forest, boosting with the proposed method. We show that the prediction accuracy of the optimal multiple-kernel SVM was much higher than other standard methods. Furthermore, we figure out the contribution of each modality in the prediction model through the kernel weights. 


\title{
IO3-2
}

\section{Using Technology Against Itself: Apps to Wean Us off Apps}

\author{
Elias Aboujaoude*
}

Stanford University, School of Medicine

eaboujaoude@stanford.edu

Background: Despite more than two decades of research into problematic Internet use, there remains considerable disagreement on what constitutes Internet-related psychopathology, much less an agreed upon treatment algorithm. Only a few psychopharmacological and psychotherapeutic interventions have been tested, with often inconsistent or disappointing results. Meanwhile, technology-mediated tools that claim to control excessive use of Internet-related technologies have grown in popularity, fueled by reports suggesting possible benefit, Silicon Valley startups seeking a new business niche and large tech companies wanting to market themselves as attuned to the negative consequences of the digital revolution.

Method: We review offerings that vary in browser, operating system and platform compatibility, and that all aim to use technology against itself.

Results: While they share similar goals as "traditional" treatments, technology-mediated interventions against problematic use of Internet-related technologies may be more efficient, scalable, and affordable. However, rigorous testing is still lacking. Conclusions: Using technology against itself may be counter-intuitive, but the popularity of these tools and their potential advantages make them worthy of research attention. Telepsychiatry, quite paradoxically, may also prove beneficial for the management of problematic use of Internet-related technologies.

\section{IO3-3}

\section{Technological based multisystemic interventions in severe MUD with the Swiss SOMOSA MediaLab-program}

\author{
Oliver Bilke-Hentsch ${ }^{1 *}$, Daniel Baumann ${ }^{1}$, Marc Bodmer ${ }^{2}$ \\ ${ }^{1}$ Modellstation SOMOSA \\ ${ }^{2}$ Cyberculturalist Zuerich \\ oliver.bilke-hentsch@somosa.ch
}

Severe MIUD often develops as a problem of focussing primarily on short motivation individual games and patterns of passive-receptive use while reducing long term creative motivational activities in groups. Therefore any specified interventions should include avariety of modern technologies to (re-)establish patterns of creative and pro-social use.

In a Swiss specialised hospital with the 25 year long mission of developing new models of treatment of severely ill adolescents a three-months- multisystemic intensive intervention $(8 \mathrm{~h} / \mathrm{d})$ is established since 2015 including: 
- Individual risc-analysis in cyber behaviour

- Daily news producing via ppt

- Daily training in office applications

- Daily Training in graphic design e.g. mangas

- Technological Programming in teams (industry 4.0)

- Complex 2- and 3D-printing of creative and industrial objects

- Drone video applications and video producing

The staff consists of social workers (2nd profession) with long gaming experiences and proven positive affinity to gaming, supervised by media-psychologists and internet media professionals such as professional game testers. It includes former graphic designers, technicians and craftsmen.

The results are impressing as the MIUD-patients do change there pathological behaviour into pro-social and employable activities thus reducing psychopathology.

\title{
IO3-4
}

\section{Cue-reactivity and craving in specific Internet-use disorders: How distal cues affect the urge to use}

\author{
Jaro Pekal ${ }^{*}$, Stephanie Antons ${ }^{2}$, Annika Brandtner ${ }^{2}$, Jennifer Janeczko², \\ Matthias Brand ${ }^{2}$ \\ ${ }^{1}$ General Psychology: Cognition and Center for Behavioral Addiction Research, University of \\ Duisburg Essen, Germany \\ ${ }^{2}$ University of Duisburg-Essen, Department of General Psychology: Cognition and Center for \\ Behavioral Addiction Research (CeBAR), Duisburg, Germany \\ jaroslaw.pekal@uni-due.de
}

Cue-Reactivity and craving are pivotal factors in the development of substance-use disorders and specific Internet-use disorders (IUD). In the field of research, the use of proximal cues is common. However, following the concept of cue-reactivity the craving should also be elicited when distal cues are present. For this purpose, a laboratory study was conducted. Each participant was allocated to a specific group based on his / her preferred application (gaming, shopping, communication, or pornography) and got to see 20 randomized pictures showing devices with application's login screens in a natural posture (e.g. smartphone hold in hand). Cues were rated regarding arousal and the triggered urge to use the certain application. Furthermore, symptom severity of specific IUDs was measured with modified versions of the short-Internet Addiction Test. Results indicate, that craving increased in the pornographic and communication condition. Moreover, the urge to use communication was significantly related to the symptom severity of an Internet-communication disorder. The results for distal cues are in line with former studies using proximal cues, showing that conditioned responses of cue-reactivity are elicited by distal cues. However, differences between applications need to be discussed. Results demonstrate similarities between substance-use disorders und specific IUDs. 


\title{
IO3-5
}

\section{Do apples not fall far from the tree? Comparing the psychiatric comorbid patterns of gambling disorder and Internet gaming disorder among Macao adult residents}

\author{
Anise M. S. Wu ${ }^{1 *}$, Juliet Honglei Chen ${ }^{1}$, Kwok Kit Tong ${ }^{1}$, Joseph T. F. Lau ${ }^{2}$ \\ ${ }^{1}$ University of Macau \\ ${ }^{2}$ Chinese University of Hong Kong \\ JulietHChen@outlook.com
}

The similarity between Gambling Disorder (GD) and Internet Gaming Disorder (IGD) has been long postulated and subsequently studied with a main focus on comparing the mechanisms. Although plenty of studies have investigated the psychiatric comorbidity of either GD or IGD, a comparison of the psychiatric comorbid patterns of the two remains an understudied field. As the first study to make such comparison among Chinese community population, we conducted an anonymous telephone survey and sampled 1000 representative Chinese adults (56\% females, aged 18-96 years). As hypothesized, we found both GD and IGD could increase one's proclivity to probable depression and probable anxiety, and vice versa. When looking closer into the altitude of increased risk, probable IGD sub-sample $(n=20)$ showed higher rates of probable depression $(30.0 \%)$ and probable anxiety $(50.0 \%)$ compared to those observed in GD sub-sample $(\mathrm{n}=19,26.3 \%$ probable depression, and $37.0 \%$ probable anxiety). These results indicate Chinese IGD gamers may be at greater risk for mental distress than GD gamblers albeit a similar psychiatric comorbid pattern between the two.

\section{IO4-1}

\section{Avoiding harmful gambling: An evidence-based set of safe gambling practices for consumers}

Nerilee Hing ${ }^{1}$, Matthew Browne ${ }^{1}$, Alex Myles Thomas Russell ${ }^{1}$, Matthew Rockloff ${ }^{1}$, Vijay Rawat ${ }^{1}$, Fiona Nicoll ${ }^{2}$, Garry Smith ${ }^{2}$

${ }^{1}$ Central Queensland University

${ }^{2}$ University of Alberta

n.hing@cqu.edu.au

Evidence-based guidelines for safe gambling practices (SGPs) that prevent gambling-related harm have been lacking. This study aimed to: 1) identify a parsimonious set of evidence-based SGPs that best predict non-harmful gambling amongst vulnerable gamblers; 2) examine how widely are they used; and 3) assess whether their use differs by gambler characteristics.

An online survey measured uptake of 43 potential SGPs, gambling harms and numerous risk factors for harmful gambling amongst 1,174 regular gamblers in Alberta Canada. Elastic net regression identified a sub-sample of 577 gamblers most vulnerable to gambling harm 
and therefore most likely to benefit from the uptake of SGPs. A second elastic net predicted gambling harm scores in the sub-sample, using the SGPs as candidate predictors. Nine SGPs best predicted non-harmful gambling. The behaviour most strongly associated with increased harm was using credit card cash advances to gamble. The behaviour most strongly associated with reduced harm was 'If I'm not having fun gambling, I stop'. These SGPs form the basis of evidence-based safe gambling guidelines which can be promoted to consumers, form the basis of self-assessment tests, be used to measure safe gambling at a population level, and inform supportive changes to policy and practice.

\title{
IO4-2
}

\section{Early trajectories of online lotteries and scratch games gamblers: growth mixture modeling coupled with latent class analysis}

\author{
Gaëlle Challet-Bouju ${ }^{1 *}$, Bastien Perrot ${ }^{2}$, Marie Grall-Bronnec ${ }^{1}$, Jean-Benoit Hardouin ${ }^{2}$ \\ ${ }^{1}$ IFAC CHU Nantes; INSERM UMR 1246, Universités Nantes \& Tours \\ ${ }^{2}$ INSERM UMR 1246, Universites Nantes et Tours; Plateforme biometrie CHU Nantes \\ gaelle.bouju@chu-nantes.fr
}

Internet is a risk factor for problem gambling, but also a great opportunity to intervene early with those who encounter difficulties. Our aim was to investigate early trajectories of gamblers over the six months following their subscription to the French online lotteries and scratch games website.

We used a two-step approach combining growth mixture modelling and latent class analysis, based on four gambling indicators: money wagered (activity), number of gambling days (activity), number of games played (risk taking) and chasing (loss of control).

We identified 5 clusters. Clusters 1 (57\%), 2 (15\%) and 3 (14\%) were characterized by zero to medium gambling activity, few chasing episodes and one game played. Cluster 4 $(10 \%)$ was characterized by medium gambling activity, with a medium number of games (1-6) and few chasing episodes. Finally, cluster 5 (5\%) was characterized by medium-high gambling activity, a high number of games (up to 17) and a high probability of chasing.

We were able to identify 2 profiles of interest for prevention: one characterised by medium activity with risk taking but no chasing, and one characterised by high activity, risk taking and chasing. The latter cluster may represent at-risk gamblers who may benefit from early preventive measures. 


\title{
IO4-3
}

\section{How coping styles, cognitive distortions, and attachment predict problem gambling among adolescents and young adults}

\author{
Filipa Calado $^{1 *}$, Joana Alexandre ${ }^{2}$, Mark D. Griffiths ${ }^{1}$ \\ ${ }^{1}$ Nottingham Trent University \\ ${ }^{2}$ ISCTE-Lisbon University Institute \\ filipa.calado2013@my.ntu.ac.uk
}

Research suggests that youth problem gambling is associated with several factors, but little is known how these factors might interact with each other in predicting this behaviour. Consequently, this study is the first to examine the mediation effect of coping styles in the relationship between attachment to parental figures and problem gambling. 988 adolescents and emerging adults were recruited to participate. The first set of analyses tested the adequacy of a model comprising biological, cognitive and family factors in predicting youth problem gambling. The second set of analyses explored the relationship between family and individual variables in problem gambling behaviour. The results of the first set of analyses demonstrated that gender, cognitive distortions, and coping styles, showed a significant predictive effect on youth problematic gambling, and the family factors of attachment and family structure did not reveal a significant influence on this behaviour. The results of the second set of analyses demonstrated that the attachment dimension of angry exerted a more indirect influence on problematic gambling, through emotion-focused coping style. This study revealed that some family variables can have a more indirect effect on youth gambling behaviour, and provided some insights in how some factors interact in predicting problem gambling.

\section{IO4-4}

\section{State of the art of studies investigating the relationship between Emotion Regulation and Gambling Disorder: preliminary results from a systematic review study}

\author{
Guyonne Rogier ${ }^{1 *}$, Patrizia Velotti ${ }^{1}$, Sara Beomonte Zobel $^{2}$, Joel Billieux ${ }^{3}$ \\ ${ }^{1}$ University of Genoa, Educational Sciences Unit \\ ${ }^{2}$ University of Rome, Sapienza, Department of Dynamic and Clinical Psychology \\ ${ }^{3}$ Addictive and Compulsive Behaviours Lab, Institute for Health and Behaviour, \\ University of Luxembourg, \\ patvelotti@gmail.com
}

Background: Despite the fact that the role played by Emotion Regulation (ER) in Gambling Disorder (GD) has been for long neglected in the gambling research field, a growing number of recent studies investigated this topic. Still, the role of ER in GD remains undermined and ignored in most treatment protocols or guidelines. This may be partially due to the 
lack of systematic examination of empirical results providing solid and definitive indications that can be translated in clinical practice. The aim of the present study is thus to conduct a systematic review on such topic.

Methods: Following the PRISMA statements, a systematic search was conducted.

Results: We identified twenty-eight studies that explored ER features and strategies in gambling. Despite most of the study supported the hypothesis of a relationship between GD and general ER deficits, results are not consistent with regard to the nature of the features characterizing ER profiles of DGs. Also, results appear to differ as a function of the sample used across studies. Finally, despite promising preliminary evidences, some features of ER appeared to be under-examined in empirical literature (e.g. rumination processes).

Discussion: Clinical implications, methodological issues and future lines of research are discussed.

\title{
IO4-5
}

\section{Development and initial validation of a new questionnaire assessing gambling craving triggers}

\author{
Aurélien Cornil ${ }^{*}$, Stephane Rothen ${ }^{2}$, Philippe de Timary ${ }^{3}$, Joel Billieux ${ }^{4}$ \\ ${ }^{1}$ Université catholique de Louvain \\ ${ }^{2}$ Geneva University Hospitals \\ ${ }^{3}$ Academic Hospital Saint Luc, Universite catholique de Louvain \\ ${ }^{4}$ University of Luxembourg, Universite catholique de Louvain, Lausanne University Hospitals \\ aurelien.cornil@uclouvain.be
}

Craving plays a pivotal role in relapse in gambling disorder patients. The Transaddiction Craving Triggers Questionnaire (TCTQ) is a 43-item self-reported instrument developed to identify craving triggers for psychoactive substances and excessive behaviors.

The present study aims to test the psychometric properties and the construct validity of the TCTQ in the context of gambling. To this end, 192 at-risk gamblers (defined by a score $\geq 3$ at the PGSI) completed the TCTQ and several items assessing gambling preferences, gambling disorder symptoms, gambling craving and urges, motives, cognitions and impulsivity traits. Principal component analysis showed that the TCTQ produced a three-factor solution that is conceptually identical to what previous results found in Alcohol Use Disorder. The three factors identified are: (1) unpleasant affect and related thoughts, (2) pleasant affect and activation, and (3) internal and external cues. Some items were removed due to floor effects and / or low loadings. Cronbach's $\alpha$ ranged from .78 to .87 , and the subscales presented positive correlations with gambling-related constructs, supporting the convergent validity.

The present study provides a gambling-adapted version of the TCTQ which can be considered a short, valid, and psychometrically sound measure to be used for research and clinical practice. 


\title{
IO5-1
}

\section{Relationships between Internet addiction and clinico-demographic and behavioral factors}

\author{
Susumu Higuchi ${ }^{3}$, Taro Muramatsu ${ }^{2}$ \\ ${ }^{1}$ Keio University School of Medicine \\ ${ }^{2}$ Department of Neuropsychiatry, Keio University School of Medicine \\ ${ }^{3}$ National Hospital Organization Kurihama Medical and Addiction Center \\ mu_salhy@yahoo.co.jp
}

Masaru Mimura ${ }^{2 *}$, Muhammad Abd El Moneam El Salhy², Takahiro Miyazaki ${ }^{2}$, Yoshihiro Noda ${ }^{2}$, Shinichiro Nakajima², Hideki Nakayama ${ }^{3}$, Satoko Mihara ${ }^{3}$, Takashi Kitayuguchi ${ }^{3}$,

Backgrounds and aims: While the Internet became an indispensable component of our contemporary life, public and academic attention is also gathered to its negative impact, namely Internet addiction (IA). Although clinico-demographic and behavioral factors are hypothetically implicated in the mechanism of IA, it still remains largely unknown how such factors are linked to IA severity. Thus, this study sought to examine relationships among IA severity and factors potentially associated with IA in Japanese students in different educational stages.

Methods: We conducted a questionnaire-based survey, which included questions about types of online activities and clinico- demographic information, the Internet Addiction Test (IAT) for IA severity, and the K6 scale for psychological distress in 3224 students at four different educational stages.

Results: IA severity was significantly positively related to the following factors: e-messaging, social networking service (SNS), games, holiday internet usage, and K6 scores, while IA severity had negative correlation with using Internet for educational purposes, age of first exposure to the Internet, and sleep duration. Age was not related to IA severity among participants using both SNS and e-messaging.

Conclusions: This study indicates the importance of comprehensive assessment of online behavior and psychological factors for further understanding of IA.

\section{IO5-2}

\section{Associations of digital media usage time with problematic smartphone use in Hong Kong Chinese adults: a population-based study}

Man Ping Wang*, Ningyuan Guo, Sai Yin Ho, Daniel Yee Tak Fong, Tai Hing Lam

The University of Hong Kong

nyguo@hku.hk

Background: Few studies have clarified which types of digital media are associated with problematic smartphone use (PSU). We studied associations of usage time on different digital media with PSU in Hong Kong Chinese adults. 
Methods: We analyzed data of 698 respondents (mean age 47.7 years, SD18.2; 43.8\% men) from a telephone survey in 2017. PSU symptoms, i.e., daily-life disturbance, withdrawal, cyber-oriented relationship, overuse, and tolerance; each range $1-6$, were assessed by the Smartphone Addiction Scale-Short Version. Hours per day on different types of digital media with computer / smartphone were self-reported. We used multivariable regression to examine the associations adjusting for sociodemographic variables.

Results: Respondents reported the longest usage time on the Internet (mean 3.0 hours, $\mathrm{SD} \pm 3.5)$, followed by instant messaging $(1.5 \pm 2.3)$ and social media $(1.1 \pm 1.8)$. Increased usage time on the Internet and instant messaging were associated with higher risks of symptoms of daily-life disturbance (adjusted beta $0.04 ; 95 \%$ CI $0.003-0.08$ ) and cyber-oriented relationship $(0.12 ; 0.04-0.21)$ respectively. Increased usage time on social media was associated with higher risks of withdrawal symptoms $(0.10 ; 0.02-0.17)$ and overall PSU risks $(0.63 ; 0.05-1.21)$.

Conclusions: Longer digital media usage time was associated with PSU, but the association varied by the types of digital media.

\title{
IO5-3
}

\section{The association of screen time with problematic smartphone use by educational attainment and household income in Hong Kong Chinese adults: a population-based study}

\author{
Man Ping Wang*, Ningyuan Guo, Sai Yin Ho, Daniel Yee Tak Fong, Tai Hing Lam \\ The University of Hong Kong \\ nyguo@hku.hk
}

Background: Studies have found the association of screen time with problematic smartphone use (PSU), but few investigated potential interaction effects of socioeconomic status. We studied the associations stratified by socioeconomic indicators among Hong Kong Chinese adults.

Methods: We analyzed data of 5063 respondents (mean age 48.1 years, SD 18.2; 45.0\% men) from a probability-based telephone survey in 2017. PSU was assessed by the Smartphone Addiction Scale-Short Version with higher scores indicating higher risks. Hours per day on the computer, tablet or smartphone were self-reported. We used multivariable regression to examine the associations stratified by educational attainment ( 3 levels) and monthly household income (2 levels), adjusting for sociodemographic variables.

Results: Screen time increased with education and income levels (both $\mathrm{P}<.001$ ). Each hour longer screen time was associated with greater PSU risks (adjusted beta $0.50 ; 95 \%$ CI $0.37-0.64$ ), but the association was stronger in respondents with lower education (adjusted beta $0.93,0.59$ and 0.33 for $\leq$ primary, secondary and tertiary education, respectively) and lower income levels (adjusted beta 0.94 and 0.40 for lower and higher income) (both P for interaction $<.05$ ).

Conclusions: Hong Kong Chinese adults with lower educational attainment and household income were more susceptible to PSU associated with screen time. 


\title{
IO5-4
}

\section{Prevalence of pathological internet use and risky behaviors among elementary school children: Toyama Safe Internet Use Workshop}

\author{
Masaaki Yamada ${ }^{*}$, Kazuo Takeuchi ${ }^{2}$, Michikazu Sekine ${ }^{3}$, Haruo Hasegawa ${ }^{3}$, \\ Takashi Tatsuse \\ ${ }^{1}$ University of Toyama, Department of Epidemiology and Health Policy \\ ${ }^{2}$ University of Hyogo \\ ${ }^{3}$ University of Toyama \\ masaakito@hotmail.com
}

Background: Little is known about the prevalence of pathological internet use and risky behaviors among elementary school children in Japan. We aimed to investigate the prevalence of problematic behaviors related to internet use in a large-scale study among children.

Methods: The survey was conducted among children who participated in Toyama Safe Internet Use Workshop in 2018. From 110 elementary schools in Toyama Prefecture 13,413 children were included. We used Young's Diagnostic Questionnaire and assessed risky behaviors related to internet, including experiences of uploading movie, charging money, causing interpersonal issues, and meeting strangers in reality.

Results: The questionnaires were returned by 13,092 children (response rate 97.6\%). The prevalence of pathological internet use was $4.2 \%$ (5.2\% in boys, $3.2 \%$ in girls). The prevalence of risky behavior was $6.6 \%(6.7 \%, 6.6 \%)$ for experiences of uploading movie, $21.6 \%$ $(31.4 \%, 11.7 \%)$ for charging money, $5.2 \%(7.1 \%, 3.2 \%)$ for causing interpersonal issues, and $2.4 \%(3.4 \%, 1.3 \%)$ for meeting strangers in reality. Older children were more likely to have problematic behaviors.

Conclusions: Pathological internet use and risky behaviors were common. Parents and health providers should be aware of these results, and education for preventing internet addiction and risky behaviors should be provided to elementary school children.

\section{IO5-5}

\section{Do Chinese and British university students use smartphones differently? A cross-cultural mixed methods study}

\author{
Zeyang Yang ${ }^{1 *}$, Kathryn Asbury², Mark D. Griffiths ${ }^{3}$ \\ ${ }^{1}$ Department of Education, University of York \\ ${ }^{2}$ University of York \\ ${ }^{3}$ Nottingham Trent University \\ yangzeyangyzy@hotmail.com
}

Background and aims: Although an increasing number of studies have focused on problematic smartphone use and smartphone addiction, few of these studies have employed both quantitative and qualitative methods or employed a cross-cultural design. A limited number 
of studies have compared eastern and western groups. The present study investigates the prevalence and causes of problematic smartphone use among Chinese and British undergraduates.

Methods: A sample of $\mathrm{n}=778$ undergraduates participated in this study (475 Chinese students and 303 British students). Students' scores on a self-report measure of problematic smartphone use were compared across country and gender. Qualitative data were analyzed using the framework approach.

Results: Chinese undergraduates reported significantly higher levels of PSU than British undergraduates, with a medium to large effect size. Females scored significantly higher than males in both groups. Chinese students reported that the sharp transition from a strictly managed high school life to a freer university life affected their level of smartphone use. Conclusions: This study indicates the importance of considering cultural and educational backgrounds when conducting studies on problematic smartphone use.

\title{
IO5-6
}

\section{European project on behavioural addictions through technologies: Findings and lessons learned}

\author{
Olatz Lopez-Fernandez*
}

Monash University

olatz.lopez-fernandez@monash.edu

According to contemporary literature and statistics, it seems global the increasing internet use and research into internet use- related addiction problems (e.g., internet addiction, online gaming addiction, disordered social networking). Furthermore, the American Psychiatric Association and the World Health Organization have also differently expanded these addictions in their respective manuals (e.g., 'gaming' as a proposed or definitive disorder into the category of 'addictions'). The 'Tech Use Disorders' project from the European Commission has provided findings which this oral communication attempts to disseminate. The methodologies applied covered all approaches to start to disentangle the general and specific internet use- related addiction problems (e.g., internet addiction versus gaming addiction). This presentation will outline some of the original findings regarding the phenomenology, development, measurement, and epidemiology of these problems. The primary outcomes will be discussed in the frame of international literature to extract some lessons learned about addictive behaviours facilitated by technologies. The last aim is to start promoting in their prevention and their education at all levels. 


\title{
IO6-1
}

\section{Impact of informative and self-appraisal pop-up messages on online gamblers}

\author{
Julie Caillon ${ }^{\prime *}$, Marie Grall-Bronnec ${ }^{2}$, Gaelle Challet-Bouju ${ }^{2}$ \\ ${ }^{1}$ Addictology Department, Nantes University Hospital \\ ${ }^{2}$ Nantes University Hospital \\ julie.caillon@chu-nantes.fr
}

Background: To prevent risks associated with online gambling, many jurisdictions proposed harm-minimisation strategies as part of a responsible gambling policy. A variety of tools are proposed, included pop-up messages. However, few studies investigated the impact of pop-up messages on the online gambling behavior.

Methods / Design: The present study evaluated the effect of informative and self-appraisal pop-up messages compared to control condition on gambling behavior and cognitive distortions in real-world gambling setting. During the experimental session, the participant was invited to gamble on his favorite gambling site as usual, with his own gambler account and his own money in our research laboratory. We included 58 online gamblers randomly assigned according to the gambler status (non problem and at-risk gamblers) and the type of game (sports and horserace betting, online poker).

Results: Compared to the control group, pop-up messages (informative and self-appraisal) did not significantly impact the gambling behavior (money wagered and time spent) immediately and at 2 weeks follow-up. We observed an impact of informative pop-up among at-risk gamblers with a decrease of the illusion of control (GRCS-IC) at 2 weeks follow-up.

Conclusions: These results suggest that informative and self-appraisal pop-up messages proposed in our study are insufficient to change gambling behavior.

\section{IO6-2}

\section{Our group therapy session has helped the people with gambling disorder}

\author{
Akihisa Iriki* \\ Osaka Psychiatric Medical Center \\ akihiriki@gmail.com
}

Background: We created an original textbook based on cognitive behavioral therapy, named GAMP (Gambling Addiction Meeting Program). We carried out group therapy sessions for outpatients.

Method: We enrolled 123 patients who diagnosed with DSM-5 as gambling disorder at our center (January 2014 to June 2018,). We compared the groups that participated in GAMP at least once, with non-participating groups. The primary endpoint was the treatment continua- 
tion rate at six months later. The protocol was approved by the institutional review board, and written informed consent was obtained from all participants.

Results: Finally, 88 patients ( 8 females) were studied, mean age $38.3 \pm 12.7$ years, and 40 patients ( 6 females) were participated in GAMP at least once. 75\% of GAMP-participating group and $16.7 \%$ of non- participating group continued treatment until 6 months later.

Discussion: What has been changed with GAMP? Our doctor's response to the gambler has changed. We now have the specialized program for gambling disorder. So, we can respond to the needs of patients and their families proudly. Patients' family members got relief. In addition, medical staff, patients and their family, everyone has been feeling a sense of accomplishment.

Conclusions: "GAMP" improved the continuation rate of gambler's treatment.

\title{
IO6-3
}

\section{Current responsible gambling strategies are not enough to prevent pathological gambling: the case of Spain}

\author{
Mariano Choliz ${ }^{1 *}$, Marta Marcos ${ }^{2}$ \\ ${ }^{1}$ Full professor \\ ${ }^{2}$ University of Valencia \\ mariano.choliz@uv.es
}

In the last years, some governments and stakeholders have implemented responsible gambling strategies. This work evidences from three points of view (theoretical, empirical and experimental) that the current responsible gambling strategies which are implemented are not effective to prevent pathological gambling and appeals to the need to regulate the gambling according to the ethical gambling principles.

Theoretically: the information is necessary, but is not enough to modify gambling habits and to prevent addictions. The main clinical symptoms of gambling disorder induce excessive gambling and are characterized by difficulty in controlling one's gambling.

Empirically: in June 2012 the online gambling was legalized in Spain. Since then, the online gambling companies have to suggest some of responsible gambling behaviors that gamblers should do in order to prevent gambling addiction. Nevertheless, the cases of online gambling addiction have been exponentially increased. Now, the online gambling represents the second cause of pathological gambling in Spain.

Experimentally: an experimental study was carried out with 40 gamblers while they are playing a real online gambling. The independent variable was the information of losses. The conclusion is that there are no differences between groups, in spite of the display of such information. 


\title{
IO6-4
}

\section{Problem gambling and gambling-related harms amongst esports bettors and skin gamblers}

\author{
Nancy Greer ${ }^{*}$ \\ Central Queensland University \\ n.greer@cqu.edu.au
}

Little is known about the newer forms of online gambling that see a convergence between video gaming and gambling, including esports betting and skin gambling. Betting on esports, which are professional video gaming competitions, is available via the majority of regulated gambling operators. An unregulated gambling subculture around esports has emerged, with virtual game items (skins) being utilised to place bets on esports and other games of chance. This has given rise to concerns that these activities are attracting younger gamblers who are more vulnerable to developing gambling problems.

This presentation describes the key findings from an online survey with 739 bettors from the USA, UK, and Canada ( $\mathrm{n}=574$ esports cash bettors; $\mathrm{n}=134$ esports skin bettors; $\mathrm{n}=$ 327 skin gamblers). Results will be presented on the demographics, problem gambling severity, and gambling-related harms by each type of gambler. The findings have the potential to inform directions for the future of esports betting and skin gambling; including further research, education and prevention campaigns, regulatory and policy change, consumer protection frameworks, and harm minimisation strategies.

\section{IO6-5}

\section{The evaluation of responsible gambling tools among real gamblers, on real gambling sites, in real time}

\author{
Mark Griffiths ${ }^{1 *}$, Michael Auer ${ }^{2}$ \\ ${ }^{1}$ Nottingham Trent University \\ ${ }^{2} \mathrm{Neccton}$ Ltd \\ mark.griffiths@ntu.ac.uk
}

Social responsibility, responsible gambling, player protection, and harm minimization in gambling have become major issues for both researchers in the gambling studies field and the gaming industry. This has been coupled with the rise of behavioural tracking technologies that allow companies to track every behavioural decision and action made by gamblers on online gambling sites, slot machines, and / or any type of gambling that utilizes player cards. This paper presents results from a series of studies carried using behavioural tracking data provided by a number of different gaming companies (particularly in relation to data concerning the use of social responsibility initiatives such as limit setting, loss-limit reminders, pop-up messaging, and personalized feedback). The studies demonstrate that tracking data can be used to evaluate responsible gambling tools and that some tools appear to be effective in reducing gambling intensity. 


\title{
IO7-2
}

\section{Recommendations for public policy and prevention of problematic online behaviours: A multi-stakeholder perspective and the common denominator}

\author{
Melina A. Throuvala ${ }^{1 *}$, Mark D. Griffiths ${ }^{1}$, Mike Rennoldson' ${ }^{2}$ Daria J. Kuss ${ }^{1}$ \\ ${ }^{1}$ International Gaming Research Unit - Nottingham Trent University, UK \\ ${ }^{2}$ Nottingham Trent University \\ melina.throuvala@ntu.ac.uk
}

Evidence suggests that problematic use of gaming and social media among adolescents is on the rise and developing into globally growing issues affecting multiple domains. How are these experienced and conceptualized among students, parents, teachers, experts and clinicians? A qualitative needs assessment investigation with the use of focus groups and individual interviews was conducted among key stakeholder groups assessing concerns, impacts, intervention needs and strategies. Results were analysed with the use of thematic analysis. Findings highlight the commonalities and the differences amongst the various groups' perspectives and provide a comprehensive account of current priorities at a public policy, education, family and individual level. An urgent call is made for i) a coordinated continuous systemic interaction between government and key societal institutions to address the multiple dimensions of the activity-specific impacts, ii) formal education that will focus on the development and practice of key skills and competencies across the developmental lifespan at a family, individual and peer level, and iii) an urgent demand for the gaming industry and social media providers to practice corporate social responsibility by endorsing a more balanced engagement with their products and services. These priorities are highlighted as critical to create worldwide impact and prevent escalation to problematic and addiction levels.

\section{IO7-3}

\section{The 'iCare for Myself' programme: \\ Testing the efficacy of a randomized controlled trial to curb distractibility and excessive smartphone and social media use}

\author{
Melina A. Throuvala ${ }^{1}$, Mark D. Griffiths ${ }^{1}$, Mike Rennoldson ${ }^{2}$, Daria J. Kuss ${ }^{1}$ \\ ${ }^{1}$ International Gaming Research Unit, Nottingham Trent University, UK \\ ${ }^{2}$ Nottingham Trent University, UK \\ melina.throuvala@ntu.ac.uk
}

Evidence suggests a growing call for prevention of excessive smartphone and social media use and the ensuing distractibility affecting academic achievement and productivity. Selfmonitoring, mindfulness and mood tracking are effectively used in interventions, yet not tested in combination for their efficacy. An evidence-based, online randomized controlled trial with the use of freely available smartphone apps was implemented amongst emergent 
adults and adolescents $(\mathrm{N}=300)$ in the UK. Participants were asked to complete pre and post online assessments measuring the efficacy of the intervention on a number of outcomes. Participants of the intervention condition were engaged in: (i) self-monitoring of social media use, (ii) voluntary abstinence from overused platforms, and (iii) brief mindfulness sessions and mood tracking, while registering daily their user experiences. Results will be analyzed with multiple regression analysis to test the hypothesized model, mediated regressions for the hypothesized mechanisms underlying use, and effect sizes to indicate the efficacy of the intervention. The current research will provide initial evidence of efficacy for the use of smartphone apps as a low-cost, first point intervention to curb distractibility and produce insight and meta-cognitive ability for online engagement. If efficacious, such interventions could serve as a complementary prevention strategy in schools or treatment settings.

\title{
IO7-4
}

\section{Associations between Internet use and emotional well-being}

\author{
Grace Wang*
}

Auckland University of Technology

gwang@aut.ac.nz

The Internet has become an integral tool in modern society, and its impact has become widespread in all aspects of our lives. Despite its benefits, evidence suggests that the harmful effects associated with addictive use of the Internet need to be addressed. This study investigated the relationship between intensity of Internet use and emotional well-being. The intensity of Internet overuse of participants $(\mathrm{n}=30 ; 15$ females, age ranged from 18 to 50) was assessed using Young's Internet Addiction Test (IAT). Depression, impulsivity and self-regulation of emotion were measured with Beck Depression Inventory (BDI), Barratt Impulsiveness Scale 11(BIS-11) and the Mini Brief Risk-Resilience Index for Screening (BRISC) respectively. The intensity of Internet use for recreational purpose was significantly positively correlated with depression, cognitive instability impulsiveness and negativity bias. In contrast, the intensity of Internet use was negatively correlated with emotion resilience. The current findings suggest that there are associations between excessive Internet use and emotional well-being. When social activities are substituted by online activities, this could lead to social withdrawal and deterioration in one's emotional well- being. 


\title{
IO7-5
}

\section{Escaping reality through videogames is linked to an implicit preference for virtual over real-life stimuli}

\author{
Jory Deleuze ${ }^{1 *}$, Pierre Maurage ${ }^{2}$, Adriano Schimmenti ${ }^{3}$, Filip Nuyens $^{4}$, Andre Melzer ${ }^{5}$, \\ Joel Billieux ${ }^{5}$ \\ ${ }^{1}$ Scientific Research and Publication Unit, le Beau Vallon (Belgium) \\ ${ }^{2}$ Catholic University of Louvain (Belgium) \\ ${ }^{3}$ University of Enna (Italy) \\ ${ }^{4}$ Nottingham Trent University (UK) \\ ${ }^{5}$ University of Luxembourg (Luxembourg) \\ jory.deleuze@beauvallon.be
}

Online gaming motives have been extensively studied in relation to both problematic and healthy video gaming. In particular, escapism (i.e., evading from real-life problems through virtual environments) is of major interest as it can constitute either a helpful or a maladaptive coping strategy.

The study aimed to test whether the escapism motive is related to a preference for the virtual environment among volunteer gamers. To go beyond the common use of self-reported questionnaires, we created a modified version of the Affect Misattribution Procedure measuring implicit attitudes toward pictures from real world vs videogames. This task, as well as questionnaires (motivations to play and the type of engagement between passion and addiction) were completed online by 273 online gamers. Results showed that participants had more positive attitudes toward pictures from virtual worlds. Furthermore, gamers playing to escape were both highly engaged in video gaming (i.e., passion) and had a more pronounced positive implicit attitude toward virtual environments.

By contributing to a better understanding of the escapism construct, our study calls for its refinement and the identification of its underlying psychological processes, as it can relate to both problematic and non-problematic uses.

\section{IO8-1}

\section{The Correlation between Gambling Disorder and Psychopathology among Medical Students in Indonesia.}

\author{
Syahrial Marwan Iskandar*
}

Medical Faculty at Universitas Indonesia, Indonesia yal.marwan67@gmail.com

Beginning with my experience of a referral case I managed where two medical students were on the verge of dropping out of school due to various life issues caused by gambling disorder, especially psychosocial issues. Gambling practices itself started as a way of escape to overcome uncomfortable feelings triggered by dissappointment. Therefore, I aim to compile 
data about gambling disorder among medical students and the association with symptoms of psychopathology.

Methods: This study used cross sectional method and involved 153 subjects. SOGS and SCL-90 instrument were used to complete this study.

Result and conclusion are in progress.

Keywords: Gambling Disorder, Psychopathology, Medical Students

\title{
IO8-2
}

\section{Therapeutical purposes for dopaminergic dysregulation syndrome and impulsive behavior in Parkinson's disease.}

\author{
Giovanni Albani ${ }^{1 *}$, Giuseppe Veneziano², Valentina Spensieri ${ }^{3}$, Antonella Peppe $^{4}$, \\ Giacomo Grassi ${ }^{5}$ \\ ${ }^{1}$ Department of Neurology, Istituto Auxologico Italiano, IRCCS Verbania (Italy) \\ *Rehabilitation Clinical Hospital "Le Terrazze", Cunardo (Varese), Italy \\ ${ }^{2}$ Rehabilitation Clinical Hospital Le Terrazze, Cunardo (Varese), Italy \\ ${ }^{3}$ Department of Dynamic and Clinic Psychology, Sapienza University, Rome, Italy \\ ${ }^{4}$ Department of Neurology and Neurorehabilitation, Santa Lucia Hospital, Rome, Italy \\ ${ }^{5}$ Department of Neurofarba, University of Florence, Italy \\ g.albani@auxologico.it
}

The L-dopa (LD) therapy is the first choice approach for Parkinson's disease (PD). The dopaminergic dysregulation syndrome (DDS) is a condition when the PD patient intakes an amount of dopaminergic drugs more than he effectively needs. This behavior leads to a drug addiction, and consequently to impulse disorders. In a previous study, we evaluated clinical and metabolic cerebral differences of PD patients with DDS. Aim of this study is to review pharmacological and non-pharmacological interventions on symptoms correlated with DDS. Opicapone, is a novel, once-daily, potent third-generation catechol-O- methyltransferase inhibitor, used as an adjunct to LD. Within the pharmacological approachs, as an alternative to neuroleptics or SSRI, it might be aimed a reduction of DDS, by an improvement of the motor response, for instance by Opicapone intake, and at end leading to a lower need of dopaminergic treatment, which in turns induce a lower LD equivalent daily dosage (LEDD). Within the non-pharmacological approaches, Repetitive Transcranial Magnetic Stimulation (rTMs) is a noninvasive procedure that uses magnetic fields to stimulate or inhibit nerve cells in the brain to improve a defined cerebral function. We will discuss findings about ongoing protocols concerning use of rTMS on impulse control disorders in PD. 


\title{
IO8-3
}

\section{Cerebral Near Infrared Spectroscopy in Parkinson's disease: an immersive virtual reality study in patients with impulse control disorders}

\author{
Giovanni Albani ${ }^{* *}$, Michelle Semonella ${ }^{2}$, Pietro Cipresso ${ }^{3}$, Fulvia Prever ${ }^{4}$, \\ Mauro Croce ${ }^{5}$, Giuseppe Veneziano ${ }^{6}$, Elisa Pedroli ${ }^{7}$, Giuseppe Riva ${ }^{3}$ \\ ${ }^{1}$ Department of Neurology, Istituto Auxologico Italiano, IRCCS Verbania (Italy) \\ Rehabilitation Clinical Hospital "Le Terrazze", Cunardo (Varese), Italy \\ ${ }^{2}$ Applied Technology for Neuro-Psychology Lab, Milan Italy \\ ${ }^{3}$ Applied Technology for Neuro-Psychology Lab, Catholic University, Milan, Italy \\ ${ }^{4}$ Addiction Clinic NHS \& Alea Association, Milan Italy \\ ${ }^{5}$ Val d'Aosta University, Italy and ASL VCO, Verbania, Italy \\ ${ }^{6}$ Rehabilitation Clinical Hospital "Le Terrazze", Cunardo (Varese) Italy \\ ${ }^{7}$ Uniecampus Novedrate Como, Italy \\ ${ }^{8}$ Department of Neurology, Istituto Auxologico Italiano, IRCCS Verbania (Italy) \\ g.albani@auxologico.it
}

Impulse control disorders (ICDs) have been associated to dopaminergic therapy in Parkinson's disease patients (PD). These disorders may include gambling disorders, compulsive buying, hypersexuality and dopaminergic dysregulation syndrome (DDS). The scope of this study is to evaluate the specific brain area activation in different during an exposure to impulse of gambling in different cathegories of PD patients and controls. Sociodemographic and clinical data including UPDRS, PDQ39, Barratt Impulsiveness Scale (BIS-11), and personality Temperament and Character Inventory-Revised (TCI-R), QUIPRs. The anxiety was assessed using STAI - Y2. All subjects were evaluated by NIRS, while performing a virtual reality test within a dedicated environment reproducing a game room (www.NeuroVr.com). The virtual environment is a closing bar, without lights, people and sound. We used Bayesian statistics provided by JASP. Preliminary data showed involvement of several structures implicated in inhibitory control, impulsivity and compulsivity. Neurocircuitries related to impulsivity, compulsivity and reward are altered by over dopaminergic stimulation in PD. At the moment, our results demonstrate that our virtual environment don't elicitate anxiety in former gamblers patients. 


\section{IO8-4}

\section{Women and gambling: the hidden group in the hidden addiction?}

Fulvia Prever ${ }^{*}$, Heather Wardle ${ }^{2}$,Helen Breen ${ }^{3}$, Adele Duncan ${ }^{4}$, Nerilee Hing, Adele Morvannou ${ }^{6}$, Jessika Spanberg ${ }^{7}$, Andrea Wohr ${ }^{8}$, Ruth Champion ${ }^{9}$

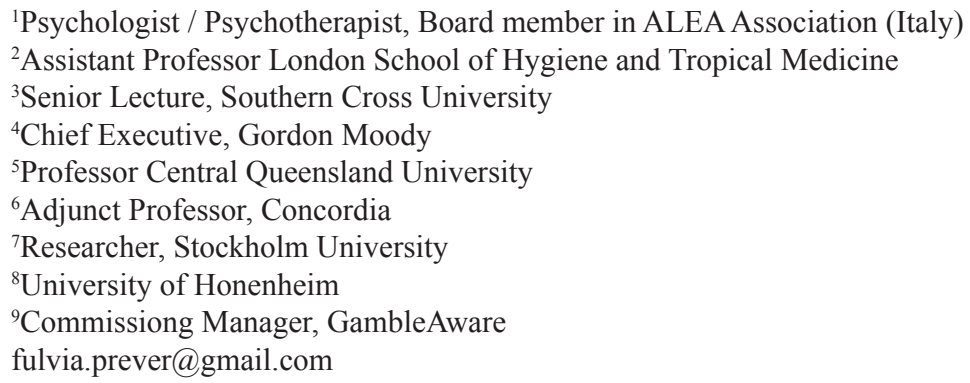

In 1980, the APA's Diagnostic and Statistics Manual IIIincluded pathological gambling for the first time. Generally attributed to the efforts of Dr. Robert Custer and his work with 'compulsive' gamblers at his clinic for (male) veterans, he described the compulsive gambler as someone who "usually goes alone. He's isolated. He's doing it to relieve some kind of psychic pain. Whether he wins or loses, just being in action relieves the pain". The tone was set, compulsive gamblers were male and evidence on which subsequent diagnostic criteria were developed were generated from men and extrapolated to women. This is a common in medical research: the experiences women are, at best, assumed to be similar to men or, at worst, considered secondary. This paper explores how these processes are manifest in the study and treatment of gambling: research privileges knowledge from men; treatment provision for women is poor; understanding of women's experiences is inadequate and there are few female advocates with lived experience of problems. We explore the reasons for this and make recommendations for how this might change, starting with an overview of the issues surrounding female gambling behaviour and present evidence from some of the (few) female specific treatment programmes for women. 


\title{
IO8-5
}

\section{Effect of an adapted physical activity program on problematic physical activity in anorexia nervosa}

\author{
Laurence Kern ${ }^{1 *}$, Nathalie Godart ${ }^{2}$, Melissa Rizk, Lilian Fautrelle ${ }^{3}$ \\ ${ }^{1}$ UFR STAPS Nanterre, CeSrm, EA2991 \\ ${ }^{2}$ Fondation Sante des etudiants de France \\ ${ }^{3}$ Universite de Toulouse, Inserm, UPS, Toulouse, France \\ lkern@parisnanterre.fr
}

Problematic physical activity (PPA) is commonly observed in patients suffering from anorexia nervosa (AN). It increases resistance to treatment programs. Recent studies demonstrated that adding a controlled and adapted physical activity (APA) program to the treatment programs had a better impact on patients suffering from AN than supressing physical activity all together. To our knowledge, there is no research on the effect of an APA program on the PPA.

Methods: 49 AN females, aged 14 to 25 years, with an average body mass index (BMI) of $16.76( \pm 2.03)$ were included. An eight-session ( $1 \mathrm{~h} 30 /$ session $)$ standardized APA program (available online) was delivered during eight consecutive weeks. The participants were evaluated using questionnaires about exercise dependence, perceived PA, and eating disorders symptoms (ED) before and after the APA program.

Results: After the completion of the APA program, we found that BMI and PA score increased ( $p<0.01)$; ED and total score of exercise dependence decreased $(\mathrm{p}<.01)$, as well as three of its dimensions (lack of control, reduction in other activities, and time).

Discussion / conclusions: The APA program seemed to help AN participants control their PPA, without any deleterious effect on BMI nor ED symptoms. The increase of PA score could be due to a better evaluation of their PA or an increase of PA linked to an increase in weight. This should be investigate in future research. This primilary data are leads as to develop a RCT.

\section{IO9-1}

\section{Dependence severity modulates precuneus features in Internet gaming disorder: functionally, morphology and effective connectivity}

\author{
Guangheng Dong ${ }^{1 *}$, Ziliang Wang ${ }^{2}$, Min Wang ${ }^{2}$, Hui Zheng ${ }^{2}$ \\ ${ }^{1}$ Hangzhou Normal University \\ ${ }^{2}$ Zhejiang Normal University \\ dongguangheng@zjnu.edu.cn
}

Background: Although the higher precuneus activation was often observed in addicted subjects when facing addiction-relevant cues, however, the recruitment of the precuneus seems inconsistent and a plausible mechanism for this remains unclear. The addiction severity might moderates precuneus features during this process, especially for Internet gaming dis- 
order (IGD). Methods: We recruited 65 IGD subjects, collected their brain responses when facing gaming cues and their brain structure data. We correlated the IGD severity with brain responses during cue-craving task, with the morphology of the precuneus, and with the effective connectivity (EC) that input / output the precuneus.

Results: In the task, IGD severity are positively correlated with the precuneus activation when facing gaming cues; In morphology, IGD severity are positively correlated with the cortical volume of precuneus; IGD severity was positively correlated with the EC from hippocampal gyrus to precuneus and negatively correlated with the EC from middle frontal gyrus to precuneus.

Conclusions: IGD severity modulates the precuneus features in IGD, and this feature could be observed functionally and morphology; The precuneus acts as a hub for the contradiction between information origin from executive control and sub- cortical cravings.

\title{
IO9-2
}

\section{Effects of imagery-induced flash-forwards and eye movements on the craving experience in excessive online gamers}

\author{
Annika Brandtner ${ }^{*}$, Jaro Pekal', Matthias Brand ${ }^{2}$ \\ ${ }^{1}$ General Psychology: Cognition and Center for Behavioral Addiction Research (CeBAR), University \\ of Duisburg-Essen, Duisburg, Germany \\ ${ }^{2}$ General Psychology: Cognition and Center for Behavioral Addiction Research (CeBAR), University \\ of Duisburg-Essen, Duisburg; 2: Erwin L. Hahn Institute for Magnetic Resonance Imaging, Essen, \\ Germany \\ annika.brandtner@uni-due.de
}

Flash-forwards describe vivid and emotionally laden mental images of future events. As it is uncertain, if such images exist in behavioral addictions, the aim of this laboratory betweenstudy was to integrate flash-forwards into the craving experience and cognitive processing in gamers with tendencies towards Internet gaming disorder (IGD). For this purpose, 77 online gamers imagined themselves playing their favorite game in the future. Afterwards, the experimental group conducted eye movements, whereas the control group performed a resting state of the eyes. Vividness and emotionality of the imagined scenario, as well as craving were measured before and after the task. Results showed no association of flash-forwards and symptom severity regarding an IGD in the overall sample. However, in problematic online gamers, flash-forwards were experienced less vivid $(\mathrm{r}=-.454, \mathrm{p}=.017)$ and less pleasurable $(\mathrm{r}=-.402, \mathrm{p}=.037)$ with advancing impairment due to gaming. Eye movements while thinking of future-oriented images lead to significantly decreased ratings of vividness ( $\mathrm{t}(39)$ $=4.29, \mathrm{p}=.001, \mathrm{~d}=0.68)$ and craving intensity $(\mathrm{t}(35)=2.74, \mathrm{p}=.009, \mathrm{~d}=0.43)$, while it did not in the resting-state condition. Results suggest different properties of flash-forwards in casual and excessive online gamers. Moreover, an eye-movement-task taxing the visual working memory seems to be beneficial to reduce craving, as craving might partly be processed visually. 
IO9-3

\title{
In-Game-Purchases and Internet Gaming Disorder. A proposal for a Maximal Revenue Per Paying User per month (MIRPPU). Why regulation in adolescents is needed?
}

\author{
Michael Dreier $^{1 *}$, Kai W. Mueller ${ }^{l}$, Manfred E. Beutel ${ }^{2}$, Daniel L. King ${ }^{3}$, Klaus Woelfling ${ }^{1}$ \\ ${ }^{1}$ Outpatient Clinic for Behavioral Addictions, Department for Psychosomatic Medicine and \\ Psychotherapy, University Medical Center Mainz, Germany \\ ${ }^{2}$ Department for Psychosomatic Medicine and Psychotherapy, University Medical Center Mainz, \\ Germany \\ ${ }^{3}$ The University of Adelaide Adelaide, Australia \\ Michael.Dreier@uni-mainz.de
}

Introduction: Prevalence in Internet Gaming Disorder (IGD) is increased, if adolescents use in-game-purchases. Nowadays, psychological functioning of in-game-purchases is still under-researched.

Method: A study investigating $\mathrm{N}=1.485$ ( $9: \mathrm{n}=676$; age: $\mathrm{M}=15.6 ; \mathrm{SD}=1.68 \& \delta^{\lambda}: \mathrm{n}=$ 809 , age: $\mathrm{M}=15.5$; $\mathrm{SD}=1.64$ ) of 12 - to 18 -year-olds addresses the question of spending behavior, psychosocial functioning and predictors for IGD in free-to- play games. Additionally, case studies resulting in excessive debts - induced by in-game-purchased - were documented.

Results: Stress is reduced via dysfunctional coping strategies such as media-related coping and behavioral disengagement. Thus, vicious circle of stress and in-game-purchases followed by more stress are hitting this vulnerable group. It is empirically indicated that adolescents spending $16 €$ or more per month suffer significantly more from emotional problems, behavioral problems, hyperactivity, problems with peers and Gaming Disorder. Additionally, these adolescents show decreased prosocial behavior and higher amounts of perceived stress.

Conclusions: Valid age verifications should be used to identify adolescent gamers. Consequently, a MIRPPU of $16 € /$ month might protect adolescent gamers from developing IGDrelated debts due to in-game-purchases (or microtransactions), which run out of control.

\section{IO9-4}

\section{Mediating Role of Self-Regulation in the Relationship between Problematic Smartphone Usage and Self-Handicapping}

\author{
Osman Tolga Aricak ${ }^{1 *}$, Mahmut Yay ${ }^{2}$, Osman T Aricak ${ }^{2}$ \\ ${ }^{1}$ Hasan Kalyoncu University \& Turkish Green Crescent Society \\ ${ }^{2}$ Hasan Kalyoncu University \\ mahmut_yay07@hotmail.com
}

In this study, it has been investigated whether self-regulation has a mediating role in the relationship between problematic smartphone usage and self-handicapping. The study was carried out with 379 participants (56\% women) aged between 20 and 29 (Mage $=23$ ). Data 
revealed that self-regulation has a mediating role in the relationship between problematic smartphone usage and self-handicapping. There are significant negative correlations between self-regulation and both self-handicapping and problematic smartphone usage. Also, there is a significant positive relationship between self-handicapping and problematic smartphone usage. In the study, the scores of the subscales of the smartphone addiction scale differed significantly according to the gender and the primary using purpose of the smartphone. Additionally, it was found that the primary using purposes of smartphones differed according to gender. It is seen that social media is the main purpose associated with the problematic use of smartphone among the primary purposes.

\section{IO9-5}

\section{Behavioural and Neurobiological evidences for diagnostic criteria specificity of Internet Gaming Disorder}

Xiaochu Zhang*, Rujing Zha

CAS Key Laboratory of Brain Function and Disease and School of Life Sciences, University of Science \& Technology of China, Hefei,

Anhui 230027, China

zxcustc@ustc.edu.cn

Introduction: Although nine criteria have been proposed for characterizing Internet Gaming Disorder (IGD) in DSM-5 and ICD-11, their specificity and usefulness remain controversial, which warrants further research. The current study combined functional MRI and behavioural data to examine the specificity of two core criteria for IGD diagnosis, including "unsuccessful attempts to control the participation in Internet games" (unsuccessful control) and "use of Internet games to escape or relieve a negative mood" (negative emotions salience attribution).

Methods: Two-hundred and twenty participants including patients with IGD (PIGD), nondependent Internet gaming participants (NIGP), and healthy controls underwent functional MRI scans at rest and during a delay-discounting task (DDT) or Iowa- gambling task (IGT), and rated the negative emotions salience attribution.

Results: Compared to healthy controls, only the PIGD but not the NIGP showed impulse control dysfunction and altered brain connectivity in the fronto-parietal network. These measures could be used to classify accurately PIGD and NIGP. In contrast, both PIGD and NIGP showed increased negative emotions salience attribution and altered connectivity in the salience network compared to controls.

Conclusions: The findings suggest that lower impulse control rather than negative emotions salience attribution may represent a feature specific to IGD. 


\title{
IO10-1
}

\section{The short Internet Addiction Test (s-IAT) and older internet users: testing measurement invariance}

\author{
Anna Ševčiková*, Lukas Blinka, David Vasek \\ Masaryk University \\ asevciko@fss.muni.cz
}

Prior research has shown that even person in older adulthood might be vulnerable internet sex addiction and report higher scores on scales measuring internet sex addiction when compared to younger internet population. There are at disposal two lines of explanations. One of them is that changes associated with ageing (e.g. retirement and later-life relationship dissolution) may pose the risk of internet sex addiction for some older adults. Another explanation could be that measurements of internet sex addiction overestimate their problematic internet use for sexual purposes. To exclude the latter, the present study aims at testing selected psychometric properties of the short Internet Addiction Test (s-IAT). Using an internet sample of 2518 Czechs $(\mathrm{M}=32.73, \mathrm{SD}=9.62 ; 73.20 \%$ male $)$, a confirmatory factor analysis and the measurement invariance testing across two groups of people aged less than $50(n=2360)$ and persons aged 50 and more $(n=158)$ were conducted. The scalar level of measurement invariance was achieved, indicating that the age groups of 50 years and above and 49 years and below do not differ significantly in terms of factor structure, factor loadings and item thresholds. The findings should be interpreted with caution due to the group's size differences.

\section{IO10-2}

\section{Alexithymia in internet gaming addiction and engagement}

\author{
Lukas Blinka*, Anna Faltynkova, Anna Sevcikova, Katerina Skarupova \\ Masaryk University \\ lukasblinka@gmail.com
}

Background and aims: A number of studies has shown a relationship between alexithymia and various addictive behaviours. This study aims at conducting a more detailed examination of alexithymia and its sub-components (difficulty to identify and describe feelings, and externally oriented thinking) with respect to internet gaming addiction and (non-pathological) engagement.

Methods: An online questionnaire was administered to experienced and active internet gamers in the Czech Republic. Only adult gamers were included in this study $(\mathrm{N}=1482$ Mage $=26,05, \mathrm{SD}=5,77,92.5 \%$ males). Addiction-Engagement Questionnaire (AEQ) and Toronto Alexithymia Scale (TAS-20) together with demographic variables and frequency and way of gaming were measured. Structured equation modelling was employed as a main analytical method. Results: Gaming addiction was significantly predicted by two subfactors 
of Alexithymia - externally oriented thinking (a weak association) and difficulty to identify emotions (a moderate association), while gaming engagement was negatively predicted by externally oriented thinking only (a weak association). Alexithymia explained $30 \%$ of variation of addiction and $8 \%$ of engagement. Conclusions: Alexithymia seems to have a good explanatory value for gaming addiction, but not for gaming engagement, pointing to a mutual exclusiveness of addiction and engagement.

\title{
IO10-3
}

\section{The Correlation between Internet Addiction and Cognitive Function among Medical Students in Indonesia}

\author{
Syahrial Marwan Iskandar* \\ Medical Faculty at Universitas Indonesia,Indonesia \\ yal.marwan67@gmail.com
}

Background: Based on World Internet User Statistic in June 2018, 55,1\% of world population are internet user and Asia was the first position rank. Indonesian Internet Service Provider Association on 2017 also showed that 54,68\% of Indonesian population is internet user. In the era of globalization, internet usage is one of the practical and effective strategies for accessing various informations. Internet is needed for accessing e-book facility as reading resources. On the other hand, the use of internet causes an addiction that affect the cognitive function and academic performance. Ge et al obtained a cognitive function decreased caused by internet addiction. The other study showed that $12 \%$ of medical students who experienced internet addiction had lower academic performance than the averages. How about the condition of medical students in Jakarta?

Methods: This study used cross sectional method and involved 153 subjects. IAT and RAVLT instrument were used to complete this study.

Result and conclusion are in progress.

Keywords: Internet, Addiction, IAT

\section{IO10-4}

\section{Craving for Violence:}

\section{The Role of Dark Personality Traits in Violent Video Game Preference}

\author{
André Melzer* \\ University of Luxembourg \\ andre.melzer@uni.lu
}

Background: Violent and antisocial video games are popular, but little is known why players are drawn to these kinds of games. This present research tested whether there is a connection between player preferences for violent games and characteristics of so called "dark" person- 
ality traits. Methods: Relying on a user-centered approach, three online studies $(\mathrm{N}=662)$ examined the role of "dark" personality traits together with a novel violent game preference short scale. Results: Study 1 and 2 indicated strong correlations between trait aggression and players' interest in explicit depictions of blood and gore and games that provide experiences of domination and antisocial behavior. In Study 3, the new scale was tested together with participants' trait aggression, trait empathy, and the Dark Triad traits of psychopathy, Machiavellianism, and narcissism. Games featuring violence and the possibility of antisocial behavior were found to meet the needs of players who lack empathy and show callous, impulsive, and exploitive (but not narcissistic) attributes. Conclusions: Findings of individual motivators for game violence significantly extend the literature that is predominantly focusing on the effects of playing these games. Apparently, game preferences and playing habits fulfill individual needs that are at least partly determined by particular, i.e. "dark", personality characteristics.

\title{
IO10-5
}

\section{Disentangling the relationship between Problematic Gaming, Emotion Dysregulation, Escapism, and Narcissism: a multiple mediation approach'}

\author{
Giardina, A. ${ }^{1}{ }^{2 *}$, Schimmenti, A. ${ }^{3}$, Lo Coco, G. ${ }^{1}$, Giordano, C. ${ }^{1}$, Billieux,.$^{2}$, \\ Di Blasi, $M .^{1}$ \\ ${ }^{1}$ University of Palermo \\ ${ }^{2}$ University of Luxembourg \\ ${ }^{3}$ KORE University of Enna \\ ale.giardi@live.it
}

Gaming disorder has been included as a mental condition in the last version of International Classification of Disease (ICD-11), despite ongoing debates regarding its conceptualization and etiology. Following a "compensatory perspective", it has been suggested that escapism and coping motives, together with emotion dysregulation, constitute key factors involved in problematic video gaming. Crucially, some dysfunctional personality factors frequently co-occurring with emotion dysregulation have to date scarcely been considered in relation to problematic video game use. Thus, this study aimed at disentangling the relationships between problematic gaming, emotion dysregulation, escapism, and narcissistic personality patterns (Vulnerable and Grandiose). In a sample of 405 World of Warcraft players, we tested a multiple mediation model in which the relationship between vulnerable / grandiose narcissism and problematic gaming was mediated by emotion dysregulation and escapism. Results confirmed our hypotheses, showing that the model for Vulnerable (but not for Grandiose) Narcissism has a very good fit. This study adds a novel insight for the understanding of mechanisms that maintain problematic gaming, suggesting that the emotion dysregulationescapism connection can be pushed by the presence of vulnerable narcissistic traits. Clinical implications are discussed.

Keywords: Gaming Disorder (GD); problematic gaming; emotion dysregulation; escapism motivations; Vulnerable Narcissism; Grandiose Narcissism. 


\title{
IO11-1
}

\section{Gender differences in problematic social media use among adolescents}

\author{
Beatrix Koronczai ${ }^{1 *}$, Gyongyi Kokonyei ${ }^{2}$, Zsolt Demetrovics $^{1}$ \\ ${ }^{1}$ ELTE Eotvos Lorand University \\ ${ }^{2}$ ELTE Eotvos Lorand University, Semmelweis University \\ koronczai.beatrix@ppk.elte.hu
}

Aim: Only few studies have examined gender differences in complex models on problematic social media use, personality traits and psychopathological symptoms. Consequently, the aim of our study was to examine the gender differences in a complex psychological explanatory model on problematic social media use.

Methods: In a cross-sectional study, secondary school students $(\mathrm{N}=2078$; mean age $=$ $16.93, \mathrm{SD}=1.65 ; 45.8 \%$ boys) were investigated with a paper pencil survey. Measurement included Bergen Social Media Addiction Scale, Brief Symptom Inventory and NEO-FFI.

Results: Based on structural equation modelling, extraversion only has a direct effect on problematic social media use, while openness to experience only has an indirect one (through global severity of distress symptoms). Neuroticism, agreeableness and conscientiousness have both direct and indirect impacts on problematic social media use. Gender differences were found; namely, introversion in females also contributed to the level of distress (indirect path to problematic social media use). Additionally, in males, conscientiousness was a protective factor only against the level of distress (indirect path), while in females, conscientiousness was a protective factor only against problematic social media use (direct path).

Conclusions: Gender invariance should be tested, for different risk factors may be important in the two genders.

\section{IO11-2}

\section{An Examination of University Student' Excessive Social Media Use and Its Relation with Personality Traits and Life Satisfaction}

\author{
Mehmet Buyukcorak ${ }^{2 *}$, Mehmet Dinc ${ }^{1}$ \\ ${ }^{1}$ Melbourne University \\ ${ }^{2}$ Istanbul Sehir University \\ mehmetdinc@gmail.com
}

The aim of our study is to examine the relation of university student' excessive social media network use and its relation with personality traits and life satisfaction. 411 university students from five different universities in Istanbul participated in this study. 208 of them were women and 203 of them were men. The measurement scales that are used in this study are; Socio-Demographic Form, Five Factor Personality Inventory and Life Satisfaction Scale. According to the findings, fourth year university students have the highest prevelance of 
social media use with $40.4 \%$. Instagram is found to be the most used social media network with the ratio of $43.6 \%$. University students, per day, on average spend 2 to 3 hours on these networks. It is found that when the score of openness to experience personality trait rises, the time that the university students spend on social media networks decreases. There was not a significant relationship is found between the daily average time spent by university students on social media networks and the other sub-scales; agreeableness, neuroticism, extraversion and conscientiousness of Five Factor Personality Inventory. It is also observed that life satisfaction of students goes up as they spend more time on social media networks.

\title{
I011-3
}

\section{The female side of gaming, gambling and internet addiction: Italian preliminary results}

\author{
Ester Di Giacomo ${ }^{1 *}$, Anders Hakansson ${ }^{2}$, Niroshani Broman', Massimo Clerici ${ }^{3}$, \\ Fulvia Prever ${ }^{4}$ \\ ${ }^{1}$ School of Medicine and Surgery, University of Milano-Bicocca (Italy)-ASST Monza, \\ Psychiatric Department (Italy) \\ 23.Lund University (Sweden) \\ ${ }^{3}$ 1. School of Medicine and Surgery, 2.University of Milano-Bicocca (Italy)-ASST Monza, \\ Psychiatric Department (Italy) \\ ${ }^{4}$ 4. ASST, Addiction Clinic, Milan (Italy) 5. Sun(n)coop, Women\&Gambling Project, Italy \\ ester.digiacomo@yahoo.com
}

Background: Problem gambling prevalence differs worldwide from $0.5 \%$ in UK (Seabury $\&$ Wardle, 2014) to 4\% in Hong Kong (Wong et al, 2003). Some of the associated risk factors in a global perspective are male sex, age under 30 years and a lower education (Abbott et al, 2014, Costes et al, 2015). We aimed at analysing Italian prevalence with a special focus on gender differences and the influence of sexual identity.

Methods: We led a survey on 1500 subjects recruited from the general population on a voluntary basis. The survey consists of a short-version of the CLiP-instrument (Gerstein et al, 2009) for gambling problems, the Game Addiction Scale (Lemmens et al, 2009) and the PRIUSS-3 (Moreno et al, 2016), for assessing problematic internet.

Results: Women (763 out of 1502) showed significant lower rate of problematic gaming (10.76 vs 11.71; Problematic internet use does not differ between genders, although women report a higher number of hours of online communication.

Conclusions: Preliminary results confirm men to be at higher risk of problematic gaming compared to women. A further evaluation will include an in-depth analysis of gender identity as influencing the risk of problematic gaming, gambling and internet use. 


\title{
IO11-4
}

\section{Time perception, a potential underlying of time loss effect among gaming disorder.}

\author{
Filip Marc Nuyens $^{1 *}$, Daria J. Kuss ${ }^{1}$, Olatz Lopez-Fernandez ${ }^{2}$, Mark D. Griffiths ${ }^{1}$ \\ ${ }^{1}$ Nottingham Trent University, Psychology Division \\ ${ }^{2}$ Monash University \\ filip.nuyens2016@my.ntu.ac.uk
}

Videogame playing is a major leisure activity worldwide and can have deleterious effects for a small proportion of gamers. One of the characteristics of disordered gamers is the time loss effect (i.e., underestimation of time spent on a game session). This paper explores the factors that can potentially explain the presence of time loss among gamers via impaired time perception. First, attention, a crucial underlying mechanism of prospective (i.e., conscious) time perception, has been shown to be better among regular gamers compared to non-gamers. This might indicate that retrospective (i.e., unconscious) time perception is more likely to be affected among gamers. Second, an altered emotion regulation coupled with an increased emotional reactivity to gaming cues could lead to interference with the gamer's time perception. Finally, entering a state flow (i.e., a total involvement in a task leading to a loss of self-consciousness) can lead to a lack of attentional resources allocated to time perception, enhancing time underestimation. In conclusion, time loss may best be explained via an interaction between an enhanced emotional reactivity to gaming cues and improved top-down attention, leading the disordered gamers not to focus on time and therefore underestimate it.

\section{IO11-5}

\section{Time loss: An impaired time perception amongst gamers?}

\author{
Filip Marc Nuyens ${ }^{1}$, Daria J. Kuss ${ }^{1}$, Olatz Lopez-Fernandez ${ }^{2}$, Mark D. Griffiths ${ }^{1}$ \\ ${ }^{1}$ Nottingham Trent University, Psychology Division \\ ${ }^{2}$ Monash University \\ filip.nuyens2016@my.ntu.ac.uk
}

Gaming has become a major leisure activity worldwide with more than two billion gamers, a small percentage of which develop problematic use. Possible negative consequences of gaming are partially associated with the time spent playing, which could be due to the time loss effect (i.e., underestimating the time spent on a game session). This study explored the potential underlying mechanisms of time loss, namely time perception. Twenty gamers and 20 non-gamers were asked to perform a lexical decision task (i.e., deciding if a string of letters is a word or non-word) and were asked to estimate the duration of the three blocks constituting the task. No difference was found regarding time perception, which led to a second experiment which explored potential factors that can affect this estimation (i.e., atten- 
tion and emotion). In the second experiment, participants were distributed across four groups varying in terms of task difficulty (i.e., easy vs. hard) and emotional level (i.e., gaming vs. neutral pictures). It was expected that gamers would exhibit a stronger underestimation when confronted with gaming pictures, the effect being strengthened by the complexity of the task.

\title{
IO11-6
}

\section{The influence of autistic trait on Gaming disorder}

\author{
Yong-Sil Kweon ${ }^{2 *}$, Seung-Yup Lee ${ }^{1}$, Hae Kook Lee ${ }^{2}$ \\ ${ }^{1}$ Department of Psychiatry, Eunpyeong St. Mary's Hospital, College of Medicine, The Catholic \\ University of Korea \\ ${ }^{2}$ Department of Psychiatry, Uijeongbu St. Mary's Hospital, College of Medicine, The Catholic \\ University of Korea
}

Background: While autism is characterized by deficits in social interactions, it is not yet clear whether the virtual environments created by gaming may appeal more to those with such deficits. However, it is not infrequent to encounter children or adolescents with pervasive developmental disorders to be involved excessively to gaming in clinical practice. Therefore, we examined whether such autistic trait may influence the course of gaming disorder.

Methods: After written informed consents from the participants and their parents, students between the ages of 10 to 16 years participated in our multicenter cohort study, which was carried out in the Seoul metropolitan area. The participants and their parents answered various psychological surveys that screen depression, attention deficit hyperactivity, autism, internet, and smartphone addiction and etc. Those with 3 or more positive gaming symptoms were included at the baseline $(\mathrm{n}=125)$. Comparisons between the first quartile and the fourth quartile were done by the total scores for autism scale at both the baseline and 6 months follow up with a p-value of 0.05 at both tails.

Results: At the baseline, the status for gaming disorder was not significantly different between the two groups. At the 6 months follow up, gamers with higher autistic trait were significantly more likely to have gaming disorder symptoms.

Discussion: Whereas attention deficit hyperactivity is an established risk factor for gaming disorder, the contribution of social deficit may have been overlooked. This finding suggests that a need to screen gaming problems for people with a pervasive developmental disorder.

Acknowledgment: This study was supported by a grant of the Korean Mental Health Technology R\&D Project, Ministry of Health \& Welfare, Republic of Korea (HM14C2603). Conflict of interest: None to declare 


\title{
IO12-1
}

\section{Addiction and Mindfulness; Pornography Addiction and Mindfulness- Based Therapy ACT}

\author{
Julie Pauline Fraumeni-McBride ${ }^{1 *}$, Jared McBride ${ }^{2}$ \\ ${ }^{1}$ Chapman University \\ ${ }^{2}$ The Steadfast Institute \\ mcbri122@mail.chapman.edu
}

Mindfulness, more specifically acceptance and commitment therapy (ACT) (Woods \& Twohig, 2008). may be the most optimal form of therapy to be used in treating problematic pornography use- use that may qualify as addiction, which falls within the realm of sex addiction (Kuzman \& Black, 2008; Hayes et. al., 2011). ACT would be beneficial to apply in a clinical setting for one-on-one therapy, coaching and sexuality workshops using the ACT model (Hayes et. al., 2011). Though some ambiguity exists on whether or not pornography addiction falls within the realm of sex addiction, there is sufficient theoretical framework available to apply addiction identifiers to problematic pornography use, which further has implications to the benefits of using ACT in treating perceived problematic or addictive pornography use (Hayes et. al., 2011).

With the given evidence to negative outcomes associated with problematic or addictive pornography use, it is important to consider the best practices and models for treating, managing and recovery from unwanted problematic pornography use (Ford, Durtschi \& Franklin, 2012).

\section{IO12-2}

\section{Adolescents compulsive sexual behavior- Family systemic approach}

\author{
Yaniv Efrati*, Mateusz Gola
}

Clinical Neuroscience Laboratory, Institute of Psychology, Polish Academy of Sciences, Warsaw, Poland; Swartz Center for Computational Neuroscience, Institute for Neural Computation, University of California San Diego, San Diego, CA, USA ypefrati@gmail.com

Background and aims: Compulsive sexual behavior (CSB) has implications for clinical and non-clinical adult populations. Disposition to CSB has been shown to influence adolescence sexual behaviors, but the development of adolescents' disposition toward CSB has not yet been examined in the family context. In the present study, we investigated whether parent-adolescent communication mediate the links between parental characteristics and adolescents' CSB.

Methods: The sample included 275 Israeli families (triad of mothers [age 34-63, M = $45.48, \mathrm{SD}=5.46$ ], fathers [age $36-83, \mathrm{M}=48.33, \mathrm{SD}=6.63$ ] and one adolescent $[48.2 \%$ boys, $51.1 \%$ girls; age $14-18, \mathrm{M}=16.23, \mathrm{SD}=1.18]$ ). Parents completed measures of psy- 
chopathology, parental self-esteem and parental self-efficacy, and adolescents completed measures of quality of sex-related communication and CSB.

Results: Results indicate that for girls, higher maternal self-esteem and lower psychopathology were linked with better sex-related communication and so with lower CSB. For boys, only parental religiosity affected the quality of sex-related communication and CSB, with religious parents having better communication than secular ones.

Discussion: The findings provide an opportunity for researchers to gain a better insight into the dynamics of familial factors in the development CSB among adolescents.

\title{
I012-3
}

\section{Aligning the Manifesto for a European research network into Problematic Usage of the Internet with the diverse needs of the professional and consumer communities affected by problematic use of pornography}

\author{
Darryl Kenneth Mead ${ }^{1 *}$, Mary Sharpe ${ }^{2}$ \\ ${ }^{1}$ The Reward Foundation and University College London \\ ${ }^{2}$ The Reward Foundation \\ darryl@rewardfoundation.org
}

How do the nine "key research priorities to advance the understanding of PUI" set out by the world's leading behavioral addiction researchers in the Manifesto for a European research network into Problematic Usage of the Internet (Fineberg et al 2018) align with the diverse needs of the different professional and consumer communities affected by problematic use of pornography?

Put simply, does the Manifesto propose conducting research that has the potential to address the issues raised by therapists, medical practitioners, counsellors and sex educators about the mental and physical health implications of problematic pornography use? Will it address the concerns of the online pornography recovery communities and members of 12 step programmes such as Sex Addicts Anonymous and Sex and Love Addicts Anonymous? Equally, will it support the sort of educational work we do in schools to help equip young people with the knowledge and skills they need to avoid PUI in all its forms?

This paper will draw upon our engagement with the different consumer groups to suggest some approaches which might further improve the alignment between the Manifesto and the needs of these groups to help reduce the negative effects of PUI from pornography viewing. 


\title{
IO12-4
}

\section{The challenges of teaching school pupils about the research on behavioural addictions}

\author{
Mary Sharpe*, Darryl K. Mead \\ The Reward Foundation \\ mary@rewardfoundation.org
}

It is vital that behavioural addiction research reaches the demographic most affected by them, namely children and adolescents. Compulsive use of internet pornography is the most difficult subject to cover. There are three main challenges to teaching this: first, teachers are reluctant to teach such a controversial subject they have not been trained to teach. This can be overcome if the lessons are about neuroplasticity, the vulnerability of the adolescent brain to supernormal stimuli and addiction. Digital detoxes, mindfulness practice and other activities can also be delivered to teach pupils about self regulation.

Second, sexual political activists have been quick to fill the limited timetable promoting lessons about consent and respect only. They ignore the research about the neuroplastic effects of regular bingeing, depleted sleep, the impacts on cognitive function and working memory from compulsive use of internet devices and the fact that half of children with compulsive sexual behavior are virgins.

Third, many parents believe they alone should teach their children about sex. Schools can help educate parents directly and via pupils to help raise awareness of the need for boundaries around internet devices. The Reward Foundation will reveal how they have met these challenges in the community.

\section{IO12-5}

\section{Problematic Sexual Internet Use among Turkish Adolescents}

\section{Osman Tolga Aricak*}

Turkish Green Crescent Society \& Hasan Kalyoncu University

tolgaaricak@gmail.com

The purpose of this study was to investigate the prevalence of problematic sexual Internet use, common behaviors of youth related to sexual use of Internet, and to identify the effect of family and friends on this issue. We surveyed 6116 adolescents aged between 12 and $18(\mathrm{Mage}=15.30)$ in Istanbul to determine if they were problematic sexual Internet users (PSIU). 247 adolescents (53 females, 194 males) were classified as problematic Internet users. One in six of participants were using Internet for sexual purposes every day, and one in four were using two or three days in a week. $9 \%$ of participants spent minimum two hours for sex when connected. $47 \%$ of participants reported that they engaged in pornographic web sites with help of their friends. Eight percent reported that their relatives, siblings, and father were effective figures. Major reasons for engaging in pornographic web sites were boredom 
(43\%) and being aroused (33\%). Data revealed that there was a significant correlation between negative critiques of fathers and mothers for behaviors of participants and duration time in sexual web sites. We also found that participants with parents who had bad relationship each other had higher Internet addiction scores than those with parents who had good relationship each other.

\title{
IO13-1
}

\section{Relationship between narcissism, self-esteem, selfie-related behaviors, and problematic smartphone use}

\author{
Qiaolei Jiang $^{1 *}$, Wenxuan Li' ${ }^{2}$,Hongdi Zhao ${ }^{2}$ \\ ${ }^{1}$ Tsinghua University \\ ${ }^{2}$ Dalian University of Technology \\ qiaoleijiang@tsinghua.edu.cn
}

Background and aims: Over the past few years, selfie-related behaviors (i.e., selfie taking, editing, and posting) have increased rapidly and become a significant part of smartphone usage. Although research has analyzed the mechanism of selfie, little is known about how individuals' underlying psychological aspects of selfie-related behaviors relate to problematic smartphone use (PSU). The objectives of this study are to identify the psychological motivations for selfie-related behaviors, and examine the relationships of two personality traits (i.e., narcissism and self-esteem) with selfie-related behaviors and PSU. Methods: A total of 685 Chinese university students (Mean $=20.53$ years, $\mathrm{SD}=1.60$ ) participated in a self-report survey, including the Narcissistic Personality Inventory, Rosenberg Self-esteem Scale, and PSU Scale. Results: The results indicate that individuals' selfie motivations include communication, archiving, and entertainment. Hierarchical regression analyses show that female individuals higher in narcissism have more selfie-related behaviors. The two personality traits play an important role in PSU, with narcissism positively associated with PSU, and selfesteem negatively associated with PSU. The association between selfie-related behaviors and PSU was also confirmed. Conclusions: Findings of this study show the psychological antecedents of selfie-related behaviors and that individuals with certain personality traits may be more vulnerable in developing PSU. 


\title{
I013-2
}

\section{Motivations, fitness tracking application usage, and smartphone addiction}

\author{
Qiaolei Jiang ${ }^{1 *}$, Xuan Zhou ${ }^{2}$ \\ ${ }^{1}$ Tsinghua University \\ ${ }^{2}$ Dalian University of Technology \\ qiaoleijiang@tsinghua.edu.cn
}

Background and aims: In recent years, various dedicated smartphone applications for fitness tracking have become the new hype. With rapid adoption and heavy use, it is worthwhile to examine their influence and potential associated risks, such as smartphone addiction. This study aims to identify the motivations for fitness tracking application usage and investigate their associations with sociodemographic factors, actual use, and smartphone addiction.

Methods: A total of 490 active WeChat Sports (the fitness tracking feature of the largest multi-purpose social media mobile application in China) users (Mean $=21.31$ years, $\mathrm{SD}=$ 3.01) completed an online survey that included their WeChat Sports activities, problematic WeChat Sports use, motivations for WeChat Sports usage, and the Smartphone Addiction Scale - Short Version (SAS-SV).

Results: Two motivations were identified, i.e., fitness and sociability, both positively associated with WeChat Sports activities, while only sociability was associated with problematic WeChat Sports use and smartphone addiction. Male users showed more WeChat Sports use, including both WeChat Sports activities and problematic use, while female users showed higher SAS-SV score.

Discussion and conclusions: The findings reveal potential associated risks of fitness tracking applications, including problematic use and smartphone addiction. Further studies could include exercise addiction into consideration.

\section{I013-3}

\section{Typology of gamers: The latent profile analysis of online gaming motives}

\author{
Orsolya Kiraly ${ }^{1 *}$, Robert Urban ${ }^{1}$, Fanni Banyai $^{2}$, Zsolt Demetrovics $^{1}$ \\ ${ }^{1}$ Institute of Psychology, ELTE Eotvos Lorand University, Budapest, Hungary \\ ${ }^{2}$ Institute of Psychology, ELTE Eotvos Lorand University, Budapest, Hungary; \\ Doctoral School of Psychology, ELTE Eotvos Lorand University, Budapest, Hungary \\ kiraly.orsolya@ppk.elte.hu
}

Background: Research has shown that people engage in online gaming for many different reasons and that certain motivations (e.g., escapism) play crucial role in the development of problematic online gaming (POG). The present study aimed to reveal reliable subtypes of 
motivational profiles and compared them in terms of game time, as well as gaming disorder, depression, anxiety and self-esteem scores.

Methods: The Motives for Online Gaming Questionnaire, the Ten-Item Internet Gaming Disorder Test, the Brief Symptom Inventory and Rosenberg's self-esteem scale were administered to a large sample of online gamers $(\mathrm{N}=5623$; 90.9\% male; mean age: 22.7 years, $\mathrm{SD}=6.5$ ) recruited via gaming-related websites.

Results: Ten motivational groups were identified with latent profile analysis that differed in intensity and also in their patterns. Groups varied most in terms of escapism scores. Furthermore, groups with high levels of escape motive, scored significantly higher in gaming disorder, depression, and anxiety, and significantly lower in self-esteem scales compared to groups with lower levels of escape motive. Gamers belonging to the group with highest scores across all motives played significantly more than any other groups.

Conclusions: These findings contribute to a better understanding of the motivational background of gaming and its relation with psychopathology and POG.

\title{
IO13-4
}

\section{Attention! Attention! -The role of attention responses in an oddball task in gamers and non-gamers}

\author{
Jaro Pekal ${ }^{*}$, Magnus Liebherr ${ }^{2}$, Annika Brandtner' ${ }^{2}$,Matthias Brand ${ }^{3}$ \\ ${ }^{1}$ General Psychology: Cognition and Center for Behavioral Addiction Research, \\ University of Duisburg Essen, Germany \\ ${ }^{2}$ University of Duisburg-Essen, Department of General Psychology: Cognition and Center \\ for Behavioral Addiction Research (CeBAR), Duisburg, Germany \\ ${ }^{3}$ University of Duisburg-Essen, Department of General Psychology: Cognition and Center \\ for Behavioral Addiction Research (CeBAR), Duisburg, Germany and Erwin L. Hahn Institute for \\ Magnetic Resonance Imaging, Essen \\ jaroslaw.pekal@uni-due.de
}

There is an ongoing debate on whether online-games negatively impact or even increase player's attention. Online-games require people's ability to pay and shift attention, react, and plan. Previous studies, however, reported reduced attention in individuals with gamingdisorder. The present study aimed to investigate differences in attention in gamers and nongamers and the influence of game-specific cues as attentional triggers. In total, 73 gamers [M $=22.73$ years, $\mathrm{SD}=4.59]$ and 41 non- gamers $[\mathrm{M}=21.78$ years, $\mathrm{SD}=2.90]$ completed the experimental study. All participants performed an auditory oddball task (90 target tones / 10 non-target tones) and were assessed with questionnaires, including screenings for symptoms of gaming- disorder. Within the group of gamers, 42 gamers performed the oddball task and watched simultaneously a video of their favorite game, 31 gamers watched a neutral video. The non-gamers performed the oddball task without a secondary task. Response time to target stimuli was significantly longer in non-gamers compared to both groups of gamers. Moreover, problematic gamers showed faster responses in the neutral condition compared to non-problematic gamers. Along with previous findings, it can be speculated that additional 
videos (independently of its content) improve attention in gamers, whereas addiction- related cues do not increase attention since it might interfere with reduced executive functions in problematic gamers.

\title{
IO13-5
}

\section{The addictive use of social networking sites, body image dissatisfaction and Body Dysmorphic Disorder: A review of psychological research}

\author{
Francesca Chloe Ryding*, Daria J. Kuss \\ Nottingham Trent University \\ francesca.ryding2018@my.ntu.ac.uk
}

Background and aims: Although body image dissatisfaction is highly influenced through excessive social networking site (SNS) use, research concerning excessive SNS usage and Body Dysmorphic Disorder (BDD) is scarce. This review intended to provide insight into SNS usage on body image dissatisfaction, in addition to highlighting how addictive SNS use may contribute to the maintenance of BDD symptomatology.

Methods: A systematic search of empirical studies was conducted to identify research on excessive SNS usage and body image dissatisfaction.

Results: Gender predicted addictive SNS use, and excessive time on SNS was associated with higher levels of appearance dissatisfaction. Greater body image dissatisfaction was experienced when SNS usage was above two hours a day. Conclusions: Increased time spent on SNS may be attributed to the motivation of using SNS for appearance comparisons. SNS appearance-based comparisons may mediate BDD symptoms, leading to addictive SNS use. Implications include implementing qualitative measures and / or a focus in the nature of SNS engagement to explore the subjective meaning of excessive SNS use for individuals. If SNS addiction intends to extend to clinical assessment, a standardised baseline needs to be established in terms of classifying addictive SNS use to allow for consistency in both research and practice.

\section{IO14-1}

\section{Harms to other from alcohol drinking on the Lahu hill tribe Thailand}

\author{
Panchapothipat $^{2}$, Phitnaree Thutsanti ${ }^{3}$ \\ ${ }^{1}$ School of Health Science, Mae Fah Luang University, Thailand \\ ${ }^{2}$ Mae Fah Luang University, Thailand \\ ${ }^{3}$ Ministry of Public Health, Thailand \\ tawatchai.api@mfu.ac.th
}

Tawatchai Apidechkul ${ }^{*}$, Onnalin Singkhorn ${ }^{2}$, Prapamon Seeprasert ${ }^{2}$, Nicharut

Alcohol use impacts both physical and mental health and also harms to other. A cross-sectional study design was conducted to estimate the prevalence of alcohol use and to explain the 
characteristics of the harms to other among the Lahu hill tribe people who lived 10 selected villages in Chiang Rai province. A validated questionnaire was used to elicit the information. Chi-square test was used to detect the associations between variables at the alpha 0.05.350 of 719 families (48.7\%) from 10 villages were identified into the category of at least a member use alcohol in their family. $27.4 \%$ of families had a conflict within family, $28.3 \%$ had a conflict with other people. Among 1,031 participants, 6.04\% were sexual harassed, people aged $\leq 15$ years and $\geq 60$ years were neglected, $1.9 \%$ were physical abuse, $5.9 \%$ were mental assaults, $4.4 \%$ were forced to use drugs from their family member who used alcohol. Harms to other from alcohol use among the Lahu were sophisticated with different causes such as low income, style of communication, jealousy, etc. Harms to other in Lahu's family have different levels of violence but needed to be solved urgently particularly in promoting quit alcohol drinking and improvement in addressing harms to other skills among family members.

\section{I014-2}

\section{Food addiction and other addictive behaviors in individuals with obesity}

Astrid Müller*, Nora M. Laskowski, Crispin Leukefeld, Carolin Hase, Martina de Zwaan

Hannover Medical School

mueller.astrid@mh-hannover.de

Aim: To investigate the relationship between food addiction (FA) and other addictive behaviors in bariatric surgery candidates. Methods: The study included 216 preoperative patients (91.7\% class 3 obesity; $80.1 \%$ women; age $\mathrm{Mdn}=44.00$ years, range $18-73)$ who filled out the Yale Food Addiction Scale 2.0 (YFAS 2.0) and standardized self-report questionnaires to measure symptoms of disorders related to substance use (alcohol and nicotine) and behavioral addictions (gambling, Internet-use, buying-shopping, hypersexual disorders and exercise dependence). Results: On a bivariate level, YFAS 2.0 FA symptoms were moderately correlated with buying-shopping disorder symptoms, and weakly correlated with Internetuse disorder symptoms. Fifty-nine patients $(27.3 \%)$ met the YFAS 2.0 threshold for FA, $1.9 \%$ for alcohol use disorder, $6.0 \%$ for nicotine use disorder, $17.3 \%$ for buying-shopping disorder, $2.3 \%$ for Internet-use disorder, and 1.4\% for hypersexual disorder. None of the patients scored above the respective questionnaire thresholds for gambling disorder or exercise dependence. Patients with vs. without YFAS 2.0 FA diagnosis did not differ with regard to prevalence estimates of addictive behaviors. Conclusions: Clinical implications, potential limitations of the findings and directions for further studies will be discussed. 


\title{
I014-3
}

\section{A cognitive-behavioral eating disorder prevention program for children: A pilot study in Polish primary schools}

\author{
Nataliia Demeshkant*, Katarzyna Potyrala, Karolina Czrewiec \\ Pedagogical University of Cracow \\ demesznat@gmail.com
}

Background: A lot of recent studies demonstrated increased number of eating disorders with some symptoms of the food addiction in children. That demands development of the appropriate prevention programs. However, in many cases they have a low effectiveness because the affected persons do not change their life habits. Investigation of the optimal delivering of the prevention programmes is important to maximize their outcomes.

Materials and Methods: The program contains four modules with environmental, digital and kinesiology content. A few trainings of the program were delivered in a school-based setting but the main part was university-setting. The objective was to reduce the sweet and fat food eating habits.

Participants: children (n 100) and childrens' parents (n 84) from schools in the Lesser Poland province, recruited through convenience sampling, participated in the questionnaire survey. Path analysis and framework analysis was applied to analyze quantitative and qualitative data.

Results: The impact of Live healthy prevention program will be discussed. Furthermore, results of the first support groups including comparing with control group will be presented.

Conclusions: Results of the initial pilot study revealed preliminary support for the feasibility of the prevention program, increases in societies' healthy habits, and decreases in food addiction symptoms.

\section{IO14-4}

\section{The Association between Posttraumatic Stress Disorder and Buying-Shopping Disorder in a Population-based Sample}

\author{
Nora Marlene Laskowski*, Martina de Zwaan, Astrid Mueller \\ Department of Psychosomatic Medicine and Psychotherapy, Hannover Medical School \\ laskowski.nora@mh-hannover.de
}

Aim: The study aimed to investigate the relationship between Posttraumatic Stress Disorder (PTSD) and Buying-Shopping Disorder (BSD) in the general adult population.

Methods: The prevalence of BSD and PTSD was examined in a representative German adult sample $(\mathrm{N}=2$ 394) by using the Compulsive Buying Scale and the Modified Posttraumatic Stress Disorder Symptom Scale.

Results: The prevalence comprised $7.0 \%$ for BSD and $5.6 \%$ for PTSD. Results show a weak significant positive correlation between the severity of PTSD and BSD in the total 
sample. Logistic regression analyses with BSD as the dependent variable and with employment, age, gender, and PTSD as predictors were conducted. Employment and PTSD were both significantly associated with BSD, with the higest odds-ratio for PTSD (OR = 4.48).

Discussion: The findings indicate an association between BSD and PTSD. The results should be taken into consideration with regard to the treatment of patients with BSD as well as for public health interventions.

\title{
I014-5
}

\section{Towards Understanding Buying Disorder: Initial Findings from Japanese National Survey}

\author{
Hanako Murase ${ }^{1 *}$, Susumu Higuchi ${ }^{1}$, Yoneatsu Ozaki², Aya Kinjo ${ }^{2}$ \\ ${ }^{1}$ Kurihama Medical and Addiction Center \\ ${ }^{2}$ Tottori University \\ hanako.shishido@gmail.com
}

Problematic buying behaviors have been historically conceptualized as impulse control disorder, compulsive buying disorder, and shopping addiction. Yet, scholars have not been able to agree on a conceptualization of this disorder, and the literature on this topic is limited. Thus, additional studies are needed in order to better understand the nature of this problem. A large national survey on problematic buying behaviors and other addictive behaviors (e.g., alcohol, internet, and gaming) was conducted in 2018 in Japan. One of the aims of this study was to examine if problematic buying behaviors belong to addiction. Another aim was to gain basic understandings on this topic, including the demographic characteristics and psychological factors (i.e., depression, hoarding, and behavioral activation and inhibition) associated with problematic shopping. Data $(\mathrm{N}=4593)$ was collected from randomly selected individuals living in Japan in 2018. In contrary to existing literature, most of the demographic variables, such as gender and income, did not predict problematic buying behaviors. Confirmatory Factor Analyses were performed to examine if problematic buying behaviors belong to addiction. Psychological factors associated with problematic shopping were uniquely distinct from their associations to other types of addictions. Initial findings from this study will be presented. 


\title{
IO15-1
}

\section{Using multiple Internet applications problematically: Differences between individuals with symptoms of one specific, multiple specific, and those without symptoms of any Internet-use disorders}

\author{
Silke M. Mueller*, Stephanie Antons, Elisa Wegmann, Matthias Brand \\ General Psychology: Cognition and Center for Behavioral Addiction Research (CeBAR), \\ University of Duisburg-Essen \\ silke.m.mueller@uni-due.de
}

Equivalent to the proposed definition of gaming disorder in the ICD-11, the uncontrolled use of applications, such as pornography, communication, gambling, and shopping, is currently discussed as other types of specific Internet-use disorders (sIUD). Theoretical and empirical approaches assume higher symptom severity to be associated with psychopathological factors and reductions in impulse control and decision making skills.

A sample of 105 males participated in a laboratory study on online pornography-use. Symptoms of sIUD were assessed using application-specific versions of the short InternetAddiction-Test. Additionally, measures of decision making, executive functions, psychopathological symptoms, use expectancies, and impulsivity were assessed.

About one third of participants showed no problematic use for any applications, 35 scored problematic at one (mostly communication), and 42 scored problematic at multiple applications. The groups showed no differences in decision making and executive functioning, but individuals with symptoms of multiple sIUD scored significantly higher on psychopathological symptoms, use expectancies, and impulsivity.

The results emphasize that individuals with multiple problematic behaviors differ from those with no or only one specific problematic usage. The findings stress the importance of comorbidities in problematic online behaviors. Future studies on sIUD should assess problematic usage across domains as possible confounding factors. 


\title{
IO15-2
}

\section{Expert appraisal of criteria for assessing gaming disorder: An international Delphi study}

\author{
Joël Billieux ${ }^{*}$, Naomi Fineberg ${ }^{2}$, Sam R. Chamberlain ${ }^{3}$, Zsolt Demetrovics ${ }^{4}$, \\ Matthias Brand ${ }^{5}$, Daniel L. King ${ }^{6}$, Lior Carmi ${ }^{7}$, Murat Yucel $^{8}$, Hans-Jurgen Rumpf ${ }^{9}$, \\ Jesus Castro-Calvo ${ }^{10}$ \\ ${ }^{1}$ University of Luxembourg \\ ${ }^{2}$ Hertfordshire Partnership University NHS Foundation trust \\ ${ }^{3}$ University of Cambridge \\ ${ }^{4}$ Eotvos Lorand University \\ ${ }^{5}$ University of Duisburg-Essen \\ ${ }^{6}$ University of Adelaide \\ ${ }^{7}$ Tel Aviv University \\ ${ }^{8}$ Monash University \\ ${ }^{9}$ University of Lubeck \\ ${ }^{10}$ University of Valencia \\ joel.billieux@uni.lu
}

Internet Gaming Disorder (IGD) has been included as a condition for further study in the DSM-5 and Gaming Disorder (GD) has been recognized as a mental condition in the ICD-11, supporting that excessive video gaming must be considered an internationally-relevant public health issue. However, validity of the proposed criteria is still a matter of debate. In this context, the aim of the current study is to use a Delphi approach to reach expert agreement concerning the clinical validity, utility, and prognostic value of the nine DSM-5 criteria and the three ICD-11 clinical guidelines proposed to define GD. In this study, 30 renowned international experts with clinical and / or research experience in GD completed iterative rounds of a Delphi survey. Each new round, experts rated proposed criteria until a pre-stablished level of agreement was achieved. Criteria rated by $80 \%$ or more experts as "extremely important" or "very important" in the diagnosis of GD were included in the final proposal. As a result, this study provides the views of experts on the most relevant, valid, and well-accepted criteria to define GD. This is important for the acceptance of criteria, however, empirical studies are needed to corroborated validity and reliability of ICD and DSM criteria. 


\title{
IO15-3
}

\section{The difference between gaming addiction and smartphone addiction in the context of temperament and character}

\author{
Sung-Gon Kim ${ }^{1 *}$, Sung Young Huh ${ }^{2}$ \\ ${ }^{1}$ Pusan National University Yangsan Hospital, Pusan National University \\ ${ }^{2}$ Pusan National University Yangsan Hospital \\ darkx2001@gmail.com
}

Objectives: Since there are no consistent diagnostic criteria for behavioral addictions yet, gaming addiction and smartphone addiction are often used confused. However, as shown in previous studies, gaming addiction and smartphone addiction have some differences. Therefore, we will investigate the differences of gaming addiction and smartphone addiction by the aspects of temperament and characteristic.

Methods: Sixty-five high school students in Busan were assessed using Temperament and Character Inventory (TCI), Smartphone Addiction Proneness Scale (SAPS) and Adaptive Game Use Scale (AUGS). Throughout the obtained data independent t-test was performed using SPSS version 22.

Results: High risk smartphone user group was nineteen, and adaptive game user group was eleven compared to normal group. The mean scores of Novelty Seeking (NS) was significantly lower $(\mathrm{p}=0.013)$ in adaptive game user group and the mean scores of Self-Directedness (SD) was significantly higher $(\mathrm{p}=0.002)$ in high risk smartphone user group than normal group. The mean scores of Harm-Avoidance (HA) was significantly higher $(p=0.05)$ in adaptive game user group than high risk smartphone user group.

Conclusions: There were significant differences in temperament and character between adaptive game user group and high risk smartphone user group. These results suggest that game addiction and smartphone addiction need to be categorized and assessed differently.

\section{IO15-4}

\section{Cyberchondria, psychopathological variables and problematic Internet use: a network analysis}

\author{
Stéphanie Baggio ${ }^{*}$, Vladan Starcevic ${ }^{2}$, David Berle ${ }^{3}$, Yasser Khazaal $^{1}$, \\ Kirupamani Viswasam ${ }^{4}$ \\ ${ }^{1}$ Geneva University Hospitals \\ ${ }^{2}$ University of Sydney \\ ${ }^{3}$ University of Technology Sydney \\ ${ }^{4}$ Nepean Hospital \\ stephanie.baggio@hcuge.ch
}

Background and aims: Although beneficial for most people, online health information seeking may be associated with heightened health anxiety and distress, especially when it is repetitive or excessive. This behavioural pattern, referred to as cyberchondria, remains elu- 
sive. The aim of this study was to ascertain whether cyberchondria is a distinct construct and shed more light on its relationships with related psychopathological variables.

Methods: Self-reported data on the severity of cyberchondria, health anxiety, symptoms of obsessive-compulsive disorder, intolerance of uncertainty, problematic Internet use, anxiety, depression, and somatic symptoms were collected from 751 participants who searched for health information online during a preceding 3-month period and were recruited from an online crowdsourcing platform. Relationships between constructs were tested using the network analysis and included a visual representation of the network and a community detection analysis.

Results: Cyberchondria emerged as a relatively distinct construct, with a low overlap with other constructs. It had stronger relationships with problematic Internet use and health anxiety than with other variables.

Conclusion: Cyberchondria was fairly well delineated from health anxiety, suggesting its conceptual independence. A close relationship between cyberchondria and problematic Internet use calls for further studies of their shared and distinct characteristics and pathogenetic mechanisms.

\title{
IO15-5
}

\section{Psychosocial determinants of Internet gaming disorder in Chinese adolescents: a cross-sectional study}

\author{
Joseph T. F. Lau ${ }^{1 *}$, Yan-Qiu Yü, Phoenix K. H. Mo' , Ji-Bin Li ${ }^{3}$ \\ ${ }^{1}$ Jockey Club School of Public Health and Primary Care, The Chinese University of Hong Kong \\ ${ }^{2}$ The Chinese University of Hong Kong \\ ${ }^{3}$ Sun Yat-sun University Cancer Center \\ 1155101049@link.cuhk.edu.hk
}

Objectives: Adolescents are particularly vulnerable to Internet gaming disorder (IGD). This study aims to investigate the relationship between psychosocial factors and IGD among Chinese adolescents.

Methods: During October and December 2018, a cross-sectional study was conducted among Grade 7-8 students from 4 junior middle schools in Guangzhou, China. Classroom surveys were conducted with self-administered questionnaires in the absence of teachers. IGD was measured using the DSM-5 diagnostic criteria and validated scales were used.

Results: Altogether 1, 362 students were surveyed among which 76.7\% played Internet games in the past 12 months. $10.7 \%$ had IGD $(n=1,045)$. Those with IGD showed significantly lower levels of refusal self-efficacy, self-control, self-esteem, resilience, and social support and had significantly higher level of impulsivity, illness perception regarding IGD, social anxiety, depressive symptoms, and loneliness than those without IGD (all $p<0.05$ ). Multivariate linear regression analysis showed that, after controlled for significant background variables, refusal self-efficacy and self-control are significant protective factors of IGD, while illness perception regarding IGD, social anxiety and depression are significantly risk factors of IGD among adolescent gamers (all $\mathrm{p}<0.05$ ). 
Conclusions: IGD prevalence was alarmingly high in Chinese adolescents, and more attention should be paid on the relationship between psychosocial factors and IGD.

\title{
IO15-6
}

\section{Videogame Addiction Research: Exploring key issues and ways forward}

\author{
Halley M. Pontes* \\ University of Tasmania \\ contactme@halleypontes.com
}

Background and Aims: Videogame addiction has recently received increased attention from official medical and psychiatric bodies such as the World Health Organization (WHO) and the American Psychiatric Association (APA) due to the latest developments in the field. Although research on videogame addiction has proliferated over the last two decades and several milestones were achieved, challenges in research still abound. Methods: The present talk is based on an analysis of the extant literature on videogame addiction research and will aim to highlight five key current issues facing the field and provide evidence-based insights on how future research may overcome such issues. Results: The literature suggests the existence of key challenges and issues in videogame addiction research regarding 1) the adoption of inconsistent definition and nomenclature; 2) absence of solid conceptual and theoretical models; 3) inconsistent assessment practices in research and clinical settings; 4) insufficient neurobiological evidence; and 5) lack of robust cross-cultural evidence. Conclusions: Overall, key challenges and issues identified may be overcome with joint effort from scholars across different scientific domains. Furthermore, potential insights on the ways forward will be considered during the talk to enable researchers consider how they may be able to assist in advancing the field further.

\section{I016-1}

\section{Buying-Shopping Disorder: Neural correlates of cue-reactivity and craving}

\author{
Patrick Trotzke ${ }^{1 *}$, Katrin Starcke ${ }^{2}$, Anya Pedersen ${ }^{3}$, Matthias Brand $^{1}$ \\ ${ }^{1}$ University of Duisburg-Essen \\ ${ }^{2}$ University of Popular Arts, Berlin \\ ${ }^{3}$ Chrisitan-Albrechts University, Kiel \\ patrick.trotzke@uni-due.de
}

Background: Individuals with buying-shopping disorder (BSD) have diminished control over their buying behavior and buy unneeded consumer goods despite experiencing negative consequences in daily life, such as severe conflicts with others, indebtedness, and even 
delinquency. Recent studies emphasize parallels to behavioral addictions and demonstrate cue-reactivity and craving, but the neural basis has not been investigated, so far.

Methods: A cue-reactivity paradigm comprising shopping-related and neutral pictures was applied using functional magnetic resonance imaging (fMRI). During fMRI scanning, individuals with BSD $(\mathrm{n}=17)$ and age / gender matched control participants watched and rated the pictures with respect to pleasantness and desires to buy. Symptom severity of BSD and craving reactions were assessed outside the scanner prior the examination.

Results: Individuals with BSD had higher baseline craving prior the investigation, rated the shopping pictures as more pleasant, and had higher desires to buy in comparison to control participants. In contrasting the shopping cues with the control cues, the BSD group had higher striatal activations compared to the control group.

Conclusions: The results demonstrate cue-reactivity and craving reactions on subjective and neural levels in individuals with BSD and emphasizes parallels to substance-use disorders and behavioral addictions, such as gambling and gaming disorder.

\title{
IO16-2
}

\section{Perceived Stress as Predictors of the Risk for Exercise Addiction in Combat and Non-Combat Army Reserves}

\author{
Attila Szabo $^{1 *}$, Amit Pinto ${ }^{1}$, Mark D Griffiths ${ }^{2}$, Aviv Weinstein ${ }^{3}$, Zsolt Demetrovics ${ }^{1}$ \\ ${ }^{1}$ ELTE Eötvös Loránd University \\ ${ }^{2}$ Nottingham Trent University \\ ${ }^{3}$ Ariel University \\ szabo.attila@ppk.elte.hu
}

Numerous individuals exercise to cope with stress. Being an army reserve could be stressful, especially for those in combat roles in a war-zone. In this inquiry we hypothesized that Israeli army reserves in a combat role will report greater stress and in coping with it will demonstrate greater risk for exercise addiction than non-combat reserves and civilian controls. Participants recruited from specifically targeted social media $(n=277$, all volunteers, fluent in English, 18 years or above, and exercising at least three times a week) answered demographic questions, the Perceived Stress Scale and Exercise Addiction Inventory on the Qualtrics online research platform. As expected, the results revealed that combat reserves scored higher on all measures than non-combat reserves and civilian controls. Perceived stress in the three groups accounted for $38.8 \%$ to $56.6 \%$ of the variance in the risk for exercise addiction. To the best knowledge of these authors, these findings are the first to demonstrate such a robust relationship between perceived stress and the risk for exercise addiction. Moreover, this is the first study to reveal difference in the risk for exercise addiction in army reserves in combat and non-combat roles. 


\title{
I016-3
}

\section{A statistical model to understand the links between leisure time exercise, problematic practice of physical exercise (PPPE), eating disorder symptoms (EDS), and the motivation to control weight}

\author{
${ }^{1}$ Université Grenoble Alpes \\ ${ }^{2}$ University Paris Nanterre \\ ${ }^{3}$ Alsana: An Eating Recovery Community \\ kotbagi.gayatri@gmail.com
}

Gayatri Kotbagi ${ }^{*}$, Yannick Morvan', Brian Cook ${ }^{3}$, Lucia Romo $^{2}$, Laurence Kern ${ }^{2}$

Aim: 1. Replicate Cook' and Hausenblas (2008) mediation model in a mixed group of students 2. Investigate the role of motivation to control weight.

Methodology: A total of $\mathrm{N}=473$ (25.4\% males and $74.6 \%$ females) students were recruited for the study. The mean age of the population was 21.9 (S.D. = 5.2). The students responded to the Exercise Dependence Scale Revised, three items of the dimension - motivation for control the weight of the Exercise Dependence Questionnaire, the SCOFF and the Godin's Leisure Time Exercise Questionnaire. The preliminary results of the statistical analysis are as follows: 1 . Exercise -- > PPPE: $\mathrm{t}(357)=3.92 ; \mathrm{p}=0.012$. Motivation to control weight -- $>$ PPPE: $\mathrm{t}(357)=6.67 ; \mathrm{p}=0.01$ 3. Interaction (exercise $\mathrm{X}$ control weight) -- > PPPE: $\mathrm{t}(357)$ $=1.98 ; \mathrm{p}=0.04$ 4. PPPE -- > EDS: $\mathrm{t}(358)=3.36 ; \mathrm{p}=0.015$. Direct effect of exercise -- $>$ EDS: $\mathrm{t}(358)=-.81 ; \mathrm{p}=.41(\mathrm{~ns})$

Conclusion: Preliminary results on a mixed group of French students replicated the full mediator effect for PPPE on the relationship between exercise and risk of EDS. Moreover, motivation to control weight did tend to moderate the relationship between exercise behaviour and PPPE. Further research is needed to examine gender effects and continue to elucidate if primary and secondary exercise dependence is evidenced. 


\title{
IO16-4
}

\section{Hooked on the Mirror: The Emergence of Exercise Addiction, Body Dysmorphic Disorder, and other Image-related psychopathological correlates in Fitness Settings}

\author{
Ornella Corazza ${ }^{I^{*}}$, Pierluigi Simonato ${ }^{2}, Z_{\text {solt Demetrovics }}^{3}$, Roisin Mooney ${ }^{1}$, \\ Katinka van de Ven ${ }^{4}$, Andres Roman-Urresterazu ${ }^{5}$, Attilio Negri ${ }^{6}$, Ilaria De Luca ${ }^{1}$, \\ Eduardo Cinosi $^{1}$, Rita Santacroce ${ }^{7}$, Giuseppe Bersani ${ }^{8}$, Giovanni Martinotti ${ }^{7}$ \\ ${ }^{1}$ Centre for Clinical \& Health Research Services, School of Life and Medical Sciences, University of \\ Hertfordshire, Hatfield, United Kingdom \\ ${ }^{2}$ Dual-Diagnosis Unit, Clinic Parco dei Tigli, Italy \\ ${ }^{3}$ Institute of Psychology, ELTE Eotvos Lorand University, Hungary \\ ${ }^{4}$ Social Policy Research Centre, University of New South Wales, Australia \\ ${ }^{5}$ Department of International Health, University of Maastricht, Netherlands \\ ${ }^{6}$ University of Milan, Italy \\ ${ }^{7}$ Department of Neuroscience, Imaging, and Clinical Science, University of Chieti Pescara, Italy \\ ${ }^{8}$ Department of Medico-Surgical Sciences and Biotechnologies, Sapienza University of Rome, Italy \\ o.corazza@herts.ac.uk
}

In a society that perpetuates the strive for a perfect appearance, a fit body has become synonymous with success, but simultaneously hard to achieve. This represents a fertile ground for the development of Exercise Addiction (EA) alongside other disorders, such as Body Dysmorphic Disorder (BDD). This study aims to explore the diffusion of EA in fitness settings and related psychopathologies in the United Kingdom, Italy, Netherlands, Hungary.

Methods: A cross-sectional sample $(\mathrm{N}=1711)$ was surveyed in gyms using the Exercise Addiction Inventory (EAI), Appearance Anxiety Inventory (AAI) and Rosenberg's Self Esteem Scale (RSE) in addition to questions on use of fitness supplements. Results: Compulsive exercise, appearance anxiety and low self-esteem were reported. 11.7\% scored over the cut off for EA. 5\% was found above the clinical cut off for BDD. 39.8\% used enhancing supplements without medical consultation (95.5\%). The latter group scored higher in both EAI and AAI. The regression model revealed a strong association between the consumption of supplements and the level of EA across the sample.

Conclusions: This study identified a high risk of EA, appearance anxiety and BDD amongst participants. The previously- unexplored association between these disorders highlights the need for informed and integrated responses targeting such vulnerable individuals. 


\title{
IO16-5
}

\section{Differences in motivations behind work addiction and excessive work}

\author{
Bernadette Kun*, Beata Bothe, Zsolt Demetrovics
}

Institute of Psychology, ELTE Eötvös Loránd University, Budapest, Hungary

kun.bernadette@ppk.elte.hu

Background: Among several myths concerning work addiction, one expresses that work addiction is equal to overwork. In contrary to this assumption, empirical studies examining this relationship have found only low or moderate correlation between work addiction and the amount of time spent with work. The differences in motivations behind work addiction and overwork have not yet been investigated, therefore our aim was to explore these possible dissimilarities. Methods: A total of 4,340 currently working adult people have been asked in a cross-sectional study. The Bergen Work Addiction Scale, the Work Addiction Risk Test, the Multidimensional Work Addiction Scale, and the 3x2 Achievement Goal Questionnaire were used to assess work addiction, work motivations, and achievement motivations. Results: Using structural equation modelling we found significant differences in work motivations and achievement motivations as predictors of work addiction and the time spent with work. Introjective, extrinsic and intrinsic work motivations, other approach, self approach and task avoidance achievement motivations were stronger predictors of work addiction than time spent with work. Conclusions: The results emphasize the dissimilarities between overwork and work addiction and highlight the main motivation backgrounds of being addicted to work and working excessively. 


\title{
IO17-1
}

\section{Short-term abstinence from problematic behaviors: A systematic review}

\author{
David P. Fernandez*, Mark D. Griffiths \\ Nottingham Trent University \\ david.fernandez2018@my.ntu.ac.uk
}

The psychological effects that result when access is prevented from engaging in a particular behavior, whether voluntarily or involuntarily, has important implications for behavioral addiction research. The present systematic review aimed to review the literature related to short-term abstinence (distinguished from abstinence as a long-term goal) from problematic behaviors. Database searches were conducted for peer-reviewed journal articles based on the following inclusion criteria: (1) short-term abstinence from problematic behaviors is a primary focus of the paper; (2) explores short-term abstinence in the context of behavioral addiction or behavioral addiction-related symptomatology; (3) published in English. Forty-nine papers were identified and included in the review, all of which examined short-term involuntary / voluntary abstinence / deprivation / restriction from problem behaviors including gambling, gaming, mobile phone use, social media use, pornography use and exercise. Findings of the review suggest that short-term abstinence could be a useful research and clinical tool to investigate addiction-related symptomatology, including, but not limited to withdrawal, craving and relapse. Short-term abstinence also shows some potential as a clinical intervention for problematic behaviors, though more empirical research is needed to understand not only its benefits but also potential counterproductive effects. 


\section{I017-2}

\section{Cross-cultural validation of the Watching TV Series Motives and Binge-Watching Engagement and Symptoms questionnaires across nine languages}

Maèva Flayelle ${ }^{*}$, Jesus Castro-Calvo ${ }^{2}$, Claus Vogele ${ }^{1}$, Robert Astur ${ }^{3}$, Rafael Ballester-Arnal ${ }^{4}$, Gaelle Challet-Bouju ${ }^{5}$, Matthias Brand ${ }^{6}$, Georgina Cardenas ${ }^{7}$, Gaetan Devos ${ }^{8}$, Hussien Elkholy ${ }^{9}$, Marie Grall-Bronnec ${ }^{5}$, Richard James ${ }^{10}$, Martha Jimenez Martinez ${ }^{11}$, Yasser Khazaal ${ }^{12}$, Daniel King ${ }^{13}$, Yueheng Liu ${ }^{14}$, Christine Lochner ${ }^{15}$, Sabine Steins-Loeber ${ }^{16}$, Jiang Long ${ }^{17}$, Marc N. Potenza ${ }^{18}$, Shahabedin Rahmatizadeh ${ }^{19}$, Adriano Schimmenti ${ }^{20}$, Dan Stein ${ }^{21}$, Istvan Toth-Kiraly ${ }^{22}$, Richard Tunney ${ }^{23}$, Saeideh Valizadeh-Haghi ${ }^{19}$, Yingying Wang $^{17}, \mathrm{Zu} \mathrm{Wei}_{\text {Zhai }}{ }^{24}$, Pierre Maurage ${ }^{25}$, Joel Billieux ${ }^{1}$

${ }^{1}$ University of Luxembourg

${ }^{2}$ University of Valencia

${ }^{3}$ University of Connecticut

${ }^{4}$ Universitat Jaume I

${ }^{5}$ Centre Hospitalier Universitaire de Nantes

${ }^{6}$ University of Duisburg-Essen

${ }^{7}$ Universidad Nacional Autonoma de Mexico

${ }^{8}$ Universite catholique de Louvain, Centre Hospitalier Le Vinatier

${ }^{9}$ Ain Shams University

${ }^{10}$ University of Nottingham

${ }^{11}$ Universidad Pedagogica y Tecnologica de Colombia

${ }^{12}$ Lausanne University Hospital

${ }^{13}$ University of Adelaide

${ }^{14}$ The Second Xiangya Hospital of Central South University

${ }^{15}$ Stellenbosch University

${ }^{16}$ Otto-Friedrich-Universitat Bamberg

${ }^{17}$ Central South University

${ }^{18}$ Yale University

${ }^{19}$ Shahid Beheshti University of Medical Sciences

${ }^{20}$ Kore University of Enna

${ }^{21}$ University of Cape Town

${ }^{22}$ Eotvos Lorand University

${ }^{23}$ Aston University

${ }^{24}$ Middlebury College

${ }^{25}$ Universite catholique de Louvain

maeva.flayelle@uni.lu

In view of the emerging psychological research on binge-watching (i.e., watching TV series episodes back-to-back), two assessment measures were developed and validated in a French-speaking population to evaluate TV series watching motives (Watching TV Series Motives Questionnaire) and binge-watching engagement and symptoms (Binge-Watching Engagement and Symptoms Questionnaire). Given their promising psychometric properties, 
these scales were subjected to cross-cultural validation in nine languages (Spanish, French, English, Hungarian, Italian, German, Arabic, Persian, Chinese). Together with additional measures (i.e., psychopathological symptoms, impulsivity, problematic Internet use), both scales were disseminated among the student population $(\mathrm{N}=12616)$ in sixteen countries. Individual confirmatory factor analyses alongside measurement invariance and correlational analyses were conducted to test the structural and external validity of the different language versions. The 4-factor and 7-factor models derived from the initial WTSMQ and BWESQ validation adequately fitted the data in each language. Both scales were invariant according to language and gender, while construct validity was evidenced by similar patterns of associations with psychopathological symptoms, impulsivity and problematic Internet use. These results suggest acceptable psychometric properties for translated WTSMQ and BWESQ, thus emphasizing their value for conducting cross-cultural research on binge-watching.

\title{
I017-3
}

\section{An examination of nomophobia among Portuguese college students}

\author{
Paulo Dias $^{1 *}$, Soraia Goncalves ${ }^{1}$, Ana-Paula Correia ${ }^{2}$ \\ ${ }^{1}$ Catholic University of Portugal \\ ${ }^{2}$ Ohio State University \\ pcdias@braga.ucp.pt
}

Nomophobia, or no mobile phone fear, is a signal of today's society where the use of phones, and specially smartphones, have a significant role in our wellbeing and adjustment to the world we live. The fear of being away from a smartphone has received public attention and recent research has shown its relationship with temperament, loneliness or psychopathology. Given the novelty of the topic and the need to deepen knowledge on this issue, the present study explored nomophobia in a Portuguese sample of college students. Sociodemographic variables, smartphone use and the predictive role of psychopathology were investigated. A sociodemographic questionnaire, the Nomophobia Questionnaire (NMP-Q; Yildirim \& Correia, 2015) and Brief Symptom Inventory (BSI; Derogatis \& Melisaratos, 1983) were administered to a sample of 337 participants between 18 and 24 years old. No gender differences or correlation with age were found, but a positive correlation with the number of smartphones and number of hours using it was established. A positive and moderate correlation was found among psychopathological symptoms, and interpersonal sensitivity turned out to be a stronger predictor of nomophobia $(\mathrm{R} 2=.284)$. Results highlight how the number of smartphones, hours using it, and the feelings of personal inadequacy and inferiority in comparison with others are relevant when explaining nomophobia. 


\title{
IO17-4
}

\section{Common myths in the behavioral addiction field}

\author{
Mark Griffiths* \\ Nottingham Trent University \\ mark.griffiths@ntu.ac.uk
}

There is no shortage of controversy and debates within the field of behavioral addiction. In this paper, a number of myths are outlined concerning various behavioral addictions. These include: (i) behavioral addictions can occur concurrently, (ii) addictions such as videogame addiction are associated with other comorbidities and are therefore not separate disorders, (iii) 'addictions' are equivalent to 'disorders' in DSM-5 and ICD-11 nomenclature, (iv) very excessive behaviors are addictions, and (v) socially condoned excessive activities and activities engaged in willfully cannot be classed as behavioral addictions. It is argued that views based on these myths depend upon how behavioral addictions are defined in the first place. It is concluded that any behavior which has severe and longstanding clinical impairment and comprising core components of addiction (i.e., salience, conflict, mood modification, tolerance, withdrawal, and relapse) should be conceptualized as a behavioral addiction.

\section{I017-5}

\section{Problematic smartphone use and Health-Related Quality of Life in Chinese adults: The Hong Kong Family and Health Information Trends Surveys (HK-FHInTS) of The Hong Kong FAMILY Project}

\author{
Tai Hing Lam $^{2}$ \\ ${ }^{1}$ School of Nursing, The University of Hong Kong \\ ${ }^{2}$ The University of Hong Kong \\ lubabezz@hku.hk
}

Man Ping Wang ${ }^{1 *}$, Sheng Zhi Zhao ${ }^{1}$, Ningyuan Guo ${ }^{2}$, Daniel Yee Tak Fong ${ }^{2}$,

Background: The association of problematic smartphone use (PSU) with health-related quality of life (HRQoL) remains unclear. We investigated how PSU is associated with HRQoL, and if the association was modified by education attainment.

Methods: We recruited 453 respondents (44.5\% male; mean age $49.88 \pm 18.02$ years; $19.7 \%$ primary or lower education) from the probability-based telephone survey (HKFHInTS) in 2017. Males scored 31 and females scored 33 in the Smartphone Addiction Scale-Short Version (ranged 10-60) were regarded as having PSU. HRQoL was measured by physical component scale (PCS) and mental component scale (MCS) (both ranged 0-100) of Short Form 12 (SF-12). Multivariable linear regression analyzed the associations between PSU and HRQoL, adjusting for sociodemographic factors. Interaction effects of education was assessed. 
Results: The prevalence of PSU was $34.4 \%$ (95\%CI $28.8 \%$ to $40.5 \%$ ). Education attainment modified the association between PSU and MCS (P for interaction < 0.05). PSU was associated with poor MCS (adjusted beta -15.93; 95\% CI -24.10 to -7.77) and lower scores in Role Emotional, Bodily Pain, Vitality, Social Functioning and Mental Health domain of SF-12 (all P $<0.05$ ) only in people with primary or lower education.

Conclusions: Chinese adults with primary or lower education attainment had much worse mental HRQoL associated with PSU. 


\title{
POSTER PRESENTATION
}

\section{P1 \\ Case Management in Buying Disorder and Borderline Personality Disorder Dual Diagnosis}

\author{
Octavian Vasiliu* \\ Dr. Carol Davila University Emergency Military Central Hospital \\ octavvasiliu@yahoo.com
}

Background and objective: Patients diagnosed with buying disorder should be screened for concomitant personality disorders, as the rate of dual diagnosis in this population tend to be underestimated. The objective of this case report is to emphasize the importance of early detection of psychiatric co-morbidities.

Methods: A 30-year old patient was diagnosed with buying disorder and received treatment with naltrexone $50 \mathrm{mg} /$ day and psychological counseling for 2 months. The evolution was not favorable, therefore a comprehensive psychological evaluation using Structured Clinical Interview for Personality Disorders and Mini-International Neuropsychiatric Interview was recommended in order to verify if additional diagnoses existed.

Results: The psychological report corroborated with a psychiatric re-evaluation supported the diagnosis of borderline personality disorder. The patient was recommended individual cognitive behavioral therapy, and a mood-stabilizer (sodium valproate $600 \mathrm{mg} /$ day) was added to his ongoing treatment. After 3 months the buying disorder severity decreased significantly (according to a self-evaluated 10-point visual analogic scale), and the financial losses were significantly lower ( $30 \%$ of the initial mean monthly expenses).

Conclusions: A comprehensive psychiatric and psychological evaluation is recommended in all patients with buying disorder, because the case manager should integrate in the treatment plan all the patient's co-morbidities.

P2

\section{Buying Disorder- The History and The Future of A Nosological Concept}

\section{Octavian Vasiliu*}

Dr. Carol Davila University Emergency Military Central Hospital

octavvasiliu@yahoo.com

Background: Buying disorder is a not a rare, marginal psychiatric entity, as epidemiological data available suggest an increase of compulsive shopping in the adult population during the last decades.

Methods: Information about buying disorder were collected from the main electronic databases (PubMed, Cochrane, Embase, PsychInfo, Thomson Reuters / Web of Science), and presented according to their historical significance. 
Results: A large number of papers dedicated to buying disorder -epidemiology, nosography, pathophysiology, evaluation and treatment- were found $(n=744)$. First attempts to scientifically describe buying disorder were realised by Bleuler and Kraepelin, who referred to oniomania and mentioned the compulsive nature of this phenomenon (in the early 20th century). No clear classification of subtypes of buying disorder and no unanimously accepted diagnosis criteria exist, after almost 100 years since the first attempt to define this disorder. The future of buying disorder lays within the determination of clinicians and researchers who have to design larger epidemiological and clinical trials in order to obtain more data about patients diagnosed with this disorder.

Conclusions: The history of the buying disorder is long (almost 100 years since the first scientific definitions) but more data should be collected from patients in good quality designed clinical and epidemiological trials.

P3

\title{
Is exercise dependence a uni or a multi-dimensional concept? A bifactor analysis of the Exercise Dependence Scale-Revised
}

\author{
Laurence Kern ${ }^{1 *}$, Gayatri Kotbagi ${ }^{2}$, Lucia Romo², Yannick Morvan $^{2}$ \\ ${ }^{1}$ UFR STAPS Nanterre, CeSrm, EA2991 \\ ${ }^{2}$ Univ Paris Nanterre, Nanterre, France \\ lkern@parisnanterre.fr
}

Introduction: The field of exercise dependence, or as we would like to call it problematic practice of physical exercise (PPPE), faces both theoretical and methodological issues (Szabo et al., 2015). For example different factorial solution were obtained for the Exercise Dependence Scale-Revised (EDS-R : Hausenblas \& Downs, 2002) : unidimensional, first and second order model. Through structural equation modelling (SEM) we aimed to test the dimensionality of EDS-R in french.

Methods: 632 students (21.2 years, SD : 4.4) (70,8\% females), completed EDS-R. A three steps SEM was conducted in Mplus : 1) unidimensional, 2) second order and 3) bi-factor model. The bifactor model allowed us to respond to the question: Are we measuring a single latent variable or a variety of different-but-related latent variables?

Results: Model fit comparisons indicated that the bi-factor was better than other model. Moreover, results indicates that most items were better measures of a general factor rather than their respective group factors (excepted for Withdrawal). Discussion: The results add to the ongoing debate in the field of behavioural addiction about how to better conceptualize and measure such a psychological construct.

Keywords: problematic practice of physical exercise, EDS-R, confirmatory factor analysis, bifactor analysis. 
P4

\title{
Psychometric validation the Korean Version of the Yale Food Addiction Scale 2.0 for Children: A Pilot Study
}

\author{
Soo-Young Bhang ${ }^{1}$, Eun Jin Park², Suk-Hyun Samuel Hwang ${ }^{3}$,Marc Potenza ${ }^{4}$, \\ Ashley N Gearhardt ${ }^{5}$, Mi-Sun Lee 6 \\ ${ }^{1}$ Department of Psychiatry, Eulji University School of Medicine, Eulji University Hospital, Seoul, \\ Korea \\ ${ }^{2}$ Department of Psychiatry College of Medicine,Inje University, Ilsan Paik Hospital, Goyang, Korea \\ ${ }^{3}$ Department of Psychology, Chonnam National University, Gwangju, Korea \\ ${ }^{4}$ Department of Psychiatry, Child Study Center, Yale University School of Medicine, New Haven, USA \\ ${ }^{5}$ Department of Psychology, University of Michigan, Ann Arbor, MI, USA \\ ${ }^{6}$ Department of Meditation Psychology, Nungin University, Hwaseong, Korea \\ dresme@hanmail.net
}

Introduction: The study aimed to examine the reliability and validity of the Korean version of the Yale Food Addiction Scale (YFAS) 2.0 for children.

Methods: In total, 135 middle school students from Korea aged 13 and 16 years old completed the questionnaire. We performed factor analysis using principal component analysis with varimax rotation. We also conducted an exploratory factor analysis (EFA) using M-Plus Version 7 to test an alternative model that demonstrated a stronger fit.

Results: The mean age of the participating adolescents (93 boys and 116 girls) was 14.84 years (standard deviation: 0.55). Most adolescents $(65.07 \%, \mathrm{n}=136)$ had a BMI z-score within the normal range (10th percentile $<$ BMI $<90$ th percentile). There is no dYFAS-2.0 mean difference between sexes (8.33 (7.69) for boys vs 9.01 (7.51) for girls, $t=-0.647$, df $=207, \mathrm{p}=0.518)$ and SES $(\mathrm{p}=0.60)$. In our CFA results, the CFI (.74), TLI (.70), RMSEA (.123), and the SRMR (.083) suggested a less than optimal fit with the one-factor model. The reliability calculated by Cronbach's alpha is 0.883 , which showed good internal reliability.

Conclusions: The Korean version of YFAS 2.0 could be an effective scale in determining food addiction in children and adolescents.

Keywords: YFAS, Food addiction, Reliability; Validity

P5

\section{Evaluation of Buying Disorder Severity Through Validated Instruments- A Review of Current Data}

\section{Octavian Vasiliu*}

Dr. Carol Davila University Emergency Military Central Hospital octavvasiliu@yahoo.com

Background and objective: Clear-cut criteria for the diagnosis of buying disorder are not available, and the newest edition of the DSM (2013) did not consider there is enough evidence to include this pathology in the chapter dedicated to substance- related and addictive 
disorders. In this context, a review of the current data available for the purpose of evaluation the severity of buying disorder manifestations could be helpful for mental health specialists in order to establish the most appropriate therapeutic interventions.

Methods: Data regarding buying disorder- severity quantification instruments were collected from the main medical electronic databases (PubMed, Cochrane, PsychInfo, Thomson Reuters / Web of Science), and presented according to their frequency of use in clinical or epidemiological trials.

Results: Compulsive Buying Scale (CBS) contains items distributed on 3 dimensions associated with compulsive buying- tendency to spend, reactivity, and post-purchase guilt. Bergen Shopping Addiction Scale (BSAS) contains items corresponding to 7 addiction criteriasalience, conflict, modification, tolerance, withdrawal, relapse, and problems. Compulsive Online Shopping Scale (COSS) was created to correspond to the DSM-5 criteria for addictive disorders.

Conclusions: A number of validated instruments are available for clinicians who may benefit from their use in the construction of a therapeutic management plan.

\section{P6}

\section{Anxiety and depressive symptomatology depending on type of games played}

\section{Isabelle Varescon ${ }^{1 *}$, Varescon Isabelle ${ }^{1}$,Sasha Mathieu ${ }^{2}$, Servane Barrault ${ }^{3}$, Paul Brunault ${ }^{4}$}

${ }^{1}$ University Paris Descartes-Sorbonne Paris Cité- LPPS

${ }^{2}$ University Paris Descartes

${ }^{3}$ University Tours

${ }^{4}$ University Tours INSERM U390 isabelle.varescon@parisdescartes.fr

Introduction: Gambling motives, cognitive distortions, anxiety and depression are involved in the development of gambling severity (Ciccarelli et al., 2017; Keough et al., 2018; Mathieu et al., 2018). The aim was to study the relationship that these variables have between them, by differentiating gamblers according to the type of games played.

Methods: 291 regular male gamblers completed sociodemographic characteristics, the South Oaks Gambling Screen, the Gambling Motives Questionnaire-Financial, the Gambling Related Cognitions Scale and the Hospital Anxiety and Depression Scale.

Results: Two gambling practices were observed: gamblers playing only skill games (skill gamblers) and gamblers playing games of skill and luck (mixed gamblers). Results showed that anxiety symptoms explained more gambling severity, coping motives and inability to stop in mixed gamblers whereas depressive symptoms predicted more these same variables in skill gamblers. However, regressions rates were much higher among mixed gamblers.

Discussion \& conclusion: These results suggest that mixed gamblers present more anxiety-coping motives, where gamblers with a plural and mixed gambling activity would play to escape or reduced anxious symptoms. So, mixed gamblers are more likely to have anxiety disorder. And gamblers, depending on games played, would have a different psychological and motivational profile, suggesting different clinical interventions. 
P7

\title{
Gambling motives and cognitive distortions among gamblers by age group
}

\author{
Isabelle Varescon ${ }^{1 *}$, Sasha Mathieu ${ }^{2}$, Servane Barrault ${ }^{3}$, Paul Brunault \\ ${ }^{1}$ University Paris Descartes-Sorbonne Paris Cité- LPPS \\ ${ }^{2}$ University Paris Descartes \\ ${ }^{3}$ University Tours \\ ${ }^{4}$ University Tours INSERM U390 \\ isabelle.varescon@parisdescartes.fr
}

Introduction: Gambling motives differ according to the age of individual (Ricijas et al., 2016). But what about cognitive distortions ? The aim was to compare gamblers by agegroup on these variables.

Method: Regular male gamblers were recruited and divided into three groups: younger aged 18 to $29(\mathrm{n}=114)$, adults aged 30 to $44(\mathrm{n}=132)$ and older aged 45 to $67(\mathrm{n}=45)$. They all completed the South Oaks Gambling Screen, the Gambling Motives QuestionnaireFinancial, the Gambling Related Cognitions Scale.

Results: Most gamblers only played skill games, between $76 \%$ and $80 \%$ per age group. If no significant difference was found between groups regarding gambling severity, younger present significantly more interpretative bias than adults and olders, more financial motives and gambling expectancies respectively than olders and adults.

Discussion: Younger gamblers would have greater expectations of the game over them, such as pleasure or peer recognition, which could result from realized and / or desired gains, and be associated to interpretative bias that supports belief that gains are due to oneself and losses to external factors. These results suggest presence of a lack of basic principles among these gamblers and the overestimation of their involvement in the outcome of the game.

P8

\section{Comparisons by gender and school type in the association between betting games and problem gambling among adolescents}

Hae-Lim Chang ${ }^{\prime *}$, Jaekyoung Lee ${ }^{2}$, RaeHyuck Lee ${ }^{3}$

${ }^{1}$ Seoul Jangsin University

${ }^{2}$ Korea Center on Gambling Problems

${ }^{3}$ Soonchunhyang University

jklee@kcgp.or.kr

This study aimed to examine the association between betting games and problem gambling among adolescents, with attention paid to comparisons by gender and school level. For this purpose, this study analyzed a sample of 3,653 adolescents from the 2015 Survey on Youth Gambling Problems. The main findings are as follows. First, experiencing online betting 
games compared to non-online betting games was associated with higher risks of being light and severe problem gambling among adolescents. Second, the associations between online betting games and problem gambling was more pronounced for male adolescents than for female adolescents. Third, the associations between online betting games and problem gambling was more pronounced for high school students than for middle school students. Based on the findings, this study suggested practical strategies to prevent problem gambling among adolescents.

\section{P9}

\section{The factors influencing the problem gambling of the suspended people of prosecution on gambling crime}

Ae Ran Park*, Sun Young Hwang, Jaekyoung Lee

Korea Center on Gambling Problems

hsy@kcgp.or.kr

This study aims to examine the factors influencing gambling problems of the suspended people of prosecution for the gambling criminals. The study was analyzed 1,584 people who participated in the education program to prevent gambling crime in 2017 , and were analysed gambling experiences, mental health problems, readiness to change gambling, gambling related cognitions, and problem gambling. As a result, the level of problem gambling was $30.8 \%$ and the level of middle risk gambling was $26.8 \%$. Also, $56.2 \%$ of the respondents were played for online gambling, and $48.8 \%$ of them showed problem gambling level. In addition, $34.7 \%$ of respondents had mental health problems. The factors influencing the problem gambling were the experience of online gambling, depression, anxiety and tension, suicide ideation and attempted suicide, readiness to change gambling, and gambling related cognitions. Based on these results, this study suggested implications for practical intervention to prevent gambling addiction and to prevent second conviction for gambling crimes.

P10

\section{Developing a Web-based Intervention for the Reduction of Problem Gambling in Switzerland: Win Back Control}

\section{Christian Baumgartner*, Michael Schaub}

Swiss Research Institute for Public Health and Addiction christian.baumgartner@isgf.uzh.ch

Background: Switzerland has a wide array of gambling opportunities. The country has 21 casinos and about 9000 lottery- vending points, which generate around 1.5 billion Swiss francs annually. This makes Switzerland one of the densest countries in the world in terms of gambling opportunities. Recent data reports a past-year gambling rate of $46.6 \%$, and a 
problem gambling prevalence rate of $1.1 \%$ in Switzerland. Evidence, however, suggests that less than $10 \%$ of pathological gamblers are in treatment at any one time with most seeking treatment only in response to a significant life crisis. Offering a web- based self-help tool could potentially reach users who otherwise would not seek traditional help.

Methods: The intervention will include the following elements: A screening questionnaire that offers personalized feedback; a gambling diary; key modules based on the approach of cognitive behavioral therapy and motivational interviewing; optional modules to deal with commonly associated comorbid mental problems; emails to send personal-tailored messages and reminders for the program to the user. The two-armed randomized controlled trial will test the effectiveness compared to a control condition with a self-help manual in reducing gambling activity in problem gamblers.

Results: Results expected Summer of 2020.

\title{
P11
}

\section{Exploring the interplay between dissociation and emotion regulation strategies among disordered gamblers: preliminary results}

\author{
Guyonne Rogier ${ }^{*}$, Jacopo Camponeschi ${ }^{2}$, Patrizia Velotti ${ }^{1}$ \\ ${ }^{1}$ University of Genoa, Educational Sciences Unit \\ ${ }^{2}$ University of Psychology of Rome, Sapienza \\ guyonne.rogier@gmail.com
}

Background: Dysfunctions in emotion regulation (ER) appear to be a central feature of Gambling Disorder (GD). Also, theoretical literature asserted that dissociation is a core process of GD used to regulate emotional arousal induced by traumatic memories (Jacobs, 1986). However, empirical literature towards dissociation is still poor and highly contrasting. Moreover, few is known towards the relationship between ER capacities and dissociation among Disordered Gamblers (DGs).

Methods: We administered to a sample of DGs $(\mathrm{n}=78)$ and a sample of community participants $(n=68)$ the Problem Gambling Index Scale (PGSI), the Dissociative Experience Scale 2nd version (DES-II) and the Emotion Regulation Questionnaire (ERQ).

Results: DGs scored higher on the Suppression subscale of the ERQ and on both the Amnesia and Absorption subscales of the DES-II but lower on the Cognitive Reappraisal subscale compared to the comparison group. The DES-II subscales correlated positively with the PGSI scores as well as with Suppression levels. The inverse pattern of results was found for the Cognitive Reappraisal dimension. Finally, we found that Dissociation predicted significantly Gambling Disorder severity above and beyond the effect of ER strategies.

Conclusions: Theoretical assumptions towards the role of dissociation and ER in GD are supported. Future line of research and clinical implications are discussed. 


\title{
P12
}

\section{Extending knowledge towards the role played by positive emotions in addiction: results from a study with disordered gamblers}

\author{
Guyonne Rogier ${ }^{*}$, Andrea Marini ${ }^{2}$, Patrizia Velotti ${ }^{1}$ \\ ${ }^{1}$ University of Genoa, Educational Sciences Unit \\ ${ }^{2}$ University of Psychology of Rome, Sapienza \\ guyonne.rogier@gmail.com
}

Background: Past research mainly focused on the role played by negative emotions in GD, neglecting processes related to positive emotionality. Recently, some authors developed a useful tool to measure dysregulation of positive emotions, the Difficulties in Emotion Regulation Scale-Positive (DERS-P, Weiss et al., 2015). In this study, we investigated the role played by dysregulation of positive emotions in GD.

Methods: We administered to a sample of Disordered Gamblers (DGs, $\mathrm{n}=95$ ) and community participants $(n=83)$ the South Oaks Gambling Screen (SOGS), the Impulsive Behavior Scale Short Form (UPPS-P) and the DERS-P.

Results: We found that DGs scored higher on the Negative and Positive Urgency subscales of the UPPS-P as well as on the Impulse and Non-acceptance subscales of the DERSP. Moreover, these scales correlated positively with the severity of GD. However, in the multiple regression only the Positive Urgency dimension resulted a significant predictor of SOGS scores. Conclusions: Our study suggests that a difficulty to accept positive emotional states in non-judgmental way is involved in GD. However, the proneness to act rashly under the influence of positive emotional states appears to be the strongest predictor of GD severity and should so be the main target of clinical interventions.

P13

\section{Adult ADHD is related to gambling severity and psychopathology in treatment-seeking problem gamblers}

\section{Samwook Choi*}

True Mind Mental Health Clinic / Korea Institute on Behavioral Addictions peaceinu@hanmail.net

Background And Aims: Previous studies showed that problem gambling and adult Attention Deficit / Hyperactivity Disorder (ADHD) often co-occur. The aim of this study was to examine whether ADHD is associated with GD and psychopathology.

Methods: 280 adult treatment-seeking problem gamblers were examined for ADHD and GD according to DSM-5. Subjects were assessed for gambling severity and co-morbid psychopathology with Problem Gambling Severity Index (PGSI), Gambling Symptom Assessment Scale (GSAS), Conners Adult ADHD Rating Scales-26 (CAARS-26), Barratt Im- 
pulsiveness Scale-11(BIS-11), Beck Depression Inventory (BDI), Beck Anxiety Inventory (BAI) and Alcohol Use Disorders Identification Test (AUDIT).

Results: In contrast with gamblers without adult ADHD $(\mathrm{n}=87)$, those with ADHD $(\mathrm{n}=$ $193)$ were reported to more severe gambling behaviors $(p<0.01)$. The prevalence of depression, anxiety, impulsivity and alcohol problem were higher in GD patients with adult ADHD than in the group without (all Ps $<0.01$ ).

Discussion And Conclusions: This study supports the link between GD and adult ADHD. This points out the necessity for screening for ADHD and other psychopathology in the treatment of GD.

Keywords: adult attention-deficit hyperactivity disorder; problem gambling; gambling disorder; psychopathology, comorbidity

\title{
P14
}

\section{Addictive behaviors and intimate partner violence: Results from a representative household survey}

\author{
Elsie Yan* \\ Hong Kong Polytechnic University \\ elsie.yan@polyu.edu.hk
}

The present study examined the association between various addictive behaviors and intimate partner violence. Data was collected from a representative sample of 1800 citizens in Hong Kong. Participants were predominately female (55\%). Most of them were married at the time of the study (61\%) and their age ranged from 18 to 97 with a mean of 50.27 (SD = 20). Engagement in addictive behaviors was common in this sample, with gambling being the most common (27.4\%), followed by alcohol (20.9\%) and tobacco use (10.4\%). Participants responded to the Revised Conflict Tactic Scale on whether they experienced any verbal and physical abusive behaviors by their partner in the past 12 months. Among those who were in a marital relationship, gambling, tobacco and alcohol use were all associated with greater likelihood of being verbally abused by their partner $(\mathrm{t}=3.15,2.59,1.34$ respectively, $\mathrm{p}<.001)$. No difference was observed for physical abuse $(\mathrm{p}>.05)$. Implications for research and practices will be discussed. 


\title{
P15
}

\section{Studying impulsivity and cognitive development function in Japanese gambling disorders}

\author{
Satoshi Furuno*, Hitomi Okada, Shunsuke Kousaka, Mitsuru Itoh, Takashi Kitayuguchi, \\ Yoshiko Ogawa, Tomoko Yonemoto, Satoko Mihara, Koutarou Nishimura, Takanobu \\ Matsuzaki, Sachio Matsushita, Susumu Higuchi \\ National Hospital Organization Kurihama Medical and Addiction Center \\ furuno.satoshi.fp@mail.hosp.go.jp
}

Objectives: Reports state that gambling disorders are frequently in coexistence with other psychiatric disorders. It is said that there is a high frequency of coexistence with developmental disorders, mainly attention-deficit / hyperactivity disorder. Additionally, the tendency of psychological characteristics such as responsiveness to stimulation and impulsivity has also been reported. Herein, we examine impulsiveness and cognitive development in Japanese gambling disorders by conducting psychological test.

Methods: Subjects (111) were diagnosed with a gambling disorder and received instructions following a psychological examination by their doctor. We used Wechsler Adult Intelligence Scale-Third Edition (WAIS-III) and Barratt Impulsiveness Scale (BIS). All subjects gave consent. Approval was given by the ethics committee of the Kurihama Medical and Addiction Center.

Results: Each index of WAIS-III was at an average level. On the contrary, the average of the total points of BIS was 73.7. Conclusions: There was no difference between each subject's WAIS-III score and the general healthy group. On the contrary, psychological test results of impulsivity were considerably higher than that of general healthy subjects. From these results, we examined the correlation between impulsivity and cognitive development.

P16

\section{Virus-mediated manipulation of radixin expression in the nucleus accumbens changes preference toward risk choice in rat gambling task}

\author{
Jeong-Hoon Kim*, Myung Ji Kwak, Bo Ram Cho, Wen Ting Cai, Wha Young Kim \\ Yonsei University College of Medicine \\ lovevic78@gmail.com
}

The ezrin-radixin-moesin (ERM) proteins play important roles in cell-shape determination. Interestingly, it was shown that ERM proteins in the nucleus accumbens (NAcc) are relevant to drugs of abuse. However, it has not been determined ever yet what functional role ERM proteins play in this site in the context of behavioral addictions. In the present study, we used rat gambling task (rGT) to train rats in a touch screen chamber to learn the relationships between 4 different light signals on the screen and accompanied reward outcomes and punishments set up with different schedules. Once animals showed a stabilized pattern of preference, they 
were bilaterally microinjected with lenti viral vectors, which contain either GFP alone, wildtype or mutant radixin gene, into the NAcc core. Two weeks after virus injection surgery, their preference of choice was re-tested in rGT chambers. Interestingly, overexpression of wild-type radixin in the NAcc changed the choice preference toward averse in a previously categorized seeking group. These effects were not observed with GFP alone. We first time showed that the preference of risk choice can be changed just by manipulating radixin expression in the NAcc core, providing a clue to molecular basis for decision-making toward risk choice behavior.

\section{P17}

\section{Social Media Addiction- The Dark Side of Post-Modern Technology}

\section{Octavian Vasiliu*}

Dr. Carol Davila University Emergency Military Central Hospital octavvasiliu@yahoo.com

Background: Nowadays, persons who are interested in online communication can access their favorite sites anytime, and from almost any place. Online communication has become for many persons a reasonably substitute for face-to-face interaction, which may increase the risk of social alienation and web-based manipulation.

Methods: An investigation was initiated to determine how much time young persons (1830 years old) are investing in online versus face-to-face communication. A number of 8 persons who presented social media addiction by self-report completed an online questionnaire focused on the preferred social media, main electronic device used, time invested in using social networking, time invested in face-to-face communication, disconfort associated with times when social media can not be accessed, and impairments related to excessive social media use.

Results: The report between technology-mediated and direct communication time was 5:1, impairment in familial and professional functioning were reported by $75 \%$ of the respondents, and the reported disconfort associated with lack of social media access was considered "very high" by $87.5 \%$.

Conclusions: Social media addiction is one of the newest behavioral addictions and one of the most important questions related to this subject is how can the mental health specialists help in preventing the expansion of this pathology. 


\title{
P18
}

\section{Depressive symptoms and suicidal behaviours in adolescent according to pattern of smartphone use: National cross-sectional study}

\author{
Joung-Sook Ahn*, Jinhee Lee, Seongho Min, Chisoo An, Min-Hyuk Kim \\ Department of Psychiatry, Yonsei University Wonju College of Medicine \\ jinh.lee95@yonsei.ac.kr
}

We aimed to investigate the association of smartphone use pattern with depressive symptoms and suicidal behaviours among adolescents. We analysed data from a nationally representative sample of Korean adolescents aged $12-18$ years $(n=62,276)$. We investigated the risks of depressive symptoms, suicide ideation, plan and attempt according to smartphone use patterns in adolescent using multiple logistic regression analyses after adjusting for confounding factors. We divided participants into four groups according to time spent of smartphone as less than $1 \mathrm{~h}, 1-2 \mathrm{~h}, 3-4 \mathrm{~h}$, and $5 \mathrm{~h}$ or more a day, and all analyses were also stratified according to the main purpose of smartphone use (social purposes / process purposes). The more time spent of a smartphone was significantly associated with depressive symptoms, suicide ideation, plan and attempt, and the association. The association between smartphone use and depressive symptoms was more prominent with smartphone use for social purposes, and the association between smartphone use and suicide ideation, plan and attempt was more prominent with smartphone use for process purposes. Our findings suggest that attention should be paid to the mental health of adolescents who spend more time on smartphone use.

\section{P19}

\section{A Study on Factors Affecting the Dependence on Mobile Phones of Korean Adolescents}

\author{
Lee Hae-Kook ${ }^{1^{*}}$, Lee Soo-Bi ${ }^{2}$, Kim Tae-Yeun ${ }^{2}$ \\ ${ }^{1}$ The univ. of Catholic \\ ${ }^{2}$ The Univ. of Chung-Ang \\ soobi88@hanmail.net
}

Smartphone has been widely distributed in Korea and the distribution rate has reached approximately $95 \%$. This issue has caused many social problems as well as physical and mental health problems of adolescents. This study explored the factors influencing the mobile phone dependence of adolescents. The subjects of the study were 1,916 grade 1 high school students. Data were obtained from the Korean Child-Youth Panel Survey (KCYPS). SPSS22.0 was used for the regression analysis. The results are as follows. First, Mobile phone-related factors have shown that males and females' mobile phone dependence were positively $(+)$ affected by 'communication with friends' and 'game using'. The 'use of media content' had positively influenced on girls' mobile phone dependence. Second, Psychological and emo- 
tional factors have found that males and females' mobile phone dependence were positively affected by 'difficulties in behavior control', 'aggression', and 'depression'. The lower level of 'social withdrawal' produced a higher level of males' mobile phones dependence. Third, In parenting attitude factor, in both sexes, 'less supervision' and 'inconsistent parenting attitude' had significant influence on mobile phone dependence. This study suggests that indepth exploration of eco-systemic factors in the prevention and intervention of mobile phone dependence of adolescents should be necessary.

\title{
P20
}

\section{Risk and protective factor of developing problematic Internet use in children}

\author{
Michio Takahashi ${ }^{1^{*}}$, Masaki Adachi², Tomoya Hirota ${ }^{3}$, Kazuhiko Nakamura ${ }^{2}$ \\ ${ }^{1}$ Research Center for Child Mental Development, Graduate School of Medicine, Hirosaki University \\ ${ }^{2}$ Hirosaki University \\ ${ }^{3}$ University of California San Francisco \\ takahashi.psy@gmail.com
}

It has not fully understood what factors predict developing future problematic Internet use in children. Therefore, we conducted a longitudinal study to examine risk and protect factors of problematic Internet use in children. Specifically, we hypothesized that depression and health-related quality of life (HRQOL) could be such factors in the development of problematic Internet use. We conducted surveys in September 2016 and 2017 for elementary and junior high school children $(\mathrm{N}=6,384)$. We used the Young Diagnostic Questionnaire to assess the severity of problematic Internet use. We also assessed depressive symptoms and HRQOL using the Depression Self-Rating Scale for Children and PedsQL, respectively. Results of multiple regression analyses demonstrated higher depressive symptoms were positively associated with more Internet use problems later a year. Whereas, physical and school functioning were negatively associated with the severity of problematic Internet use one year later. These results suggest that higher depressive symptoms can be risk factors, and higher physical and school functioning can be protective factors for developing problematic Internet use in children. Therefore, it is important to consider to target these factors in developing future intervention strategies to prevent developing Internet use in elementary and junior high school students. 


\title{
P21
}

\section{Tangled up in blue: response inhibition in Problematic Facebook Use}

\author{
Tania Moretta*, Sarlo Michela, Palomba Daniela, Buodo Giulia \\ Department of General Psychology, University of Padova \\ tania.moretta@phd.unipd.it
}

A deficit in inhibitory control is thought to represent a core feature in individuals with Problematic Facebook Use (PFU). We investigated the modulation of Facebook-related stimuli on response inhibition in individuals with PFU by recording the event-related potentials during an emotional Go / NoGo Task. Nineteen-nine Problematic Facebook users and 22 Non-problematic users were recruited based on their score on the Problematic Facebook Use Scale. The Go / NoGo task included Facebook-related pictures and unpleasant, pleasant, and neutral pictures selected from the International Affective Picture System. The amplitude of the NoGo-N2 and the NoGo-P3 components were measured as reflecting the detection of response conflict and response inhibition, respectively. Reaction times to Go stimuli and accuracy (omissions and commissions) were also measured. Whereas the two groups did not differ in response speed, problematic users made overall more commission errors than Non-problematic users. In Problematic users only, the NoGo-N2 was significantly larger to Facebook stimuli than to all other emotional categories, whereas the NoGo-P3 was similarly reduced to Facebook-related, pleasant and neutral vs. unpleasant stimuli. These findings suggest that conflict monitoring is enhanced, and inhibitory processing is reduced, when individuals with PFU must withhold action in the presence of Facebook stimuli.

\section{P22}

\section{Internet gaming disorder, treatment-seeking and attention deficit / hyperactivity disorder}

\author{
Doug Hyun Han ${ }^{2 *}$, Sujin Bae ${ }^{l}$, Taeyoung Choi ${ }^{2}$, Tae Ho Kim ${ }^{3}$, Sun Mi Kim ${ }^{1}$, \\ Vladan Starcervic ${ }^{4}$ \\ ${ }^{1}$ Chung Ang University Hospital \\ ${ }^{2}$ Dae Gu Catholic University Hospital \\ ${ }^{3}$ Kunkook University Hospital \\ ${ }^{4}$ Medical School, Nepean Clinical School \\ hduk70@gmail.com
}

Background and aims: The relationship between Internet gaming disorder (IGD) and attention deficit / hyperactivity disorder (ADHD) is complex. The purpose of this study was to compare the prevalence of IGD and ADHD in two groups of gamers, depending on whether or not they sought treatment.

Methods: The first group of study participants consisted of 58 individuals who sought treatment for their gaming-related problems, while the other included 33 gamers who did not 
seek treatment. They were interviewed to establish the presence of IGD based on the DSM-5 criteria and administered various instruments, including the Adult ADHD Self-Report Scale. Results: Of 58 treatment-seeking participants, 38 (65.5\%) were diagnosed with IGD. Of these 38 individuals with IGD, $33(86.8 \%)$ were also diagnosed with ADHD. Of 33 gamers who did not seek treatment, $14(42.4 \%)$ were diagnosed with IGD. Of these 14 IGD individuals who did not seek treatment, 6 (42.9\%) were also diagnosed with ADHD.

Conclusions: Seeking treatment for gaming-related problems does not necessarily reflect the presence of IGD and co-occurring ADHD, although the proportion of gamers with IGD and ADHD co-occurring with IGD was significantly higher in a treatment- seeking sample than in a sample of gamers who did not seek treatment.

\title{
P23
}

\section{Gaming behaviors of non-school based youths in Thailand which affect their health}

\author{
Kanittha Thaikla* \\ Resraech Institute for Health Sciences, Chiang Mai University \\ kthaikla@gmail.com
}

Background: Popular games among non-school based youths are Music Online Game, Real-time strategy, Action First Person Shooters Games, and Sport \& Racing Games. This study aims at examining factors affecting game addiction of non-school based youths.

Methods: We have conducted a cross-sectional survey of non-school based youths through venue-based sampling and a private interview with 1,373 youths.

Results: $77.0 \%$ of youths play games. Among them, $58.1 \%$ play games in the past 1 year, 3-4 days per week on average. They play games mostly at home / dormitory. $34.5 \%$ of youths are addicted to game. Male youths have 1.6 times higher risk for game addiction than female youths. Youths who lack of care and warmth and affection from family tend to have 3.23 times higher risk for game addiction when compared to those without game addiction problems.

Conclusions: Crucial factors for game addiction are family problems and over-attachment to friends or boyfriend / girlfriend. 


\title{
P24
}

\section{Cognitive function in patients of internet gaming disorder}

\author{
Ichiro Sora ${ }^{1 *}$, Noriomi Eguchi ${ }^{1}$, Atsushi Ohuchi ${ }^{1}$, Naruhisa Yamaki ${ }^{1}$, Naoko Iwamoto ${ }^{1}$, \\ Shiho Miyazawa ${ }^{2}$,Hiroko Tamiya ${ }^{1}$, Akitoyo Hishimoto ${ }^{1}$ \\ ${ }^{1}$ Department of Psychiatry, Kobe University Graduate School of Medicine \\ ${ }^{2}$ Department of Biological Psychiatry, Tohoku University Graduate School of Medicine \\ eguchi03@med.kobe-u.ac.jp
}

It has been well reported that patients with drug addiction show cognitive dysfunction. We investigated whether cognitive dysfunction is also observed in patients with Internet Gaming Disorder (IGD), which is behavioral, non-drug addiction.

We administered MATRICS Cognitive Consensus Battery Japanese version (MCCB-J) to IGD patients diagnosed according to DSM-5 diagnostic criteria and healthy controls. The score of social cognition in IGD group was lower than that in healthy control group, whereas other domains, such as processing speed, attention / vigilance, working memory, verbal learning and problem solving were not different between IGD and control group.

Our results suggest that cognitive domains except social cognition were maintained, suggesting the possibility that obvious neuronal deficit does not occur in patients with IGD, unlike patients with drug addiction. Furthermore, low score of social cognition in our IGD patients may indicate that social dysfunction could be one of the factors of IGD. Further investigations are required to reveal the pathophysiology of IGD.

\section{P25}

\section{Problematic use of online pornography and psychopathological distress}

\author{
Manuel Mennig*, Silke Sophia Tennie, Antonia Barke \\ Philipps-University Marburg \\ manuel.mennig@uni-marburg.de
}

Online pornography (OP) is a very popular Internet application. As with other Internet applications, its use can become problematic. In this study we used the Online Pornography Disorder Questionnaire (OPDQ) - an instrument that adapted the official criteria of Internet Gaming Disorder to OP - to measure problematic use. First indications point to a link between problematic use of OP and psychopathological distress and general functional impairment. The aim of this study was to investigate whether users with an unproblematic and problematic use of OP differ with regard to their psychopathological distress.

An online sample of German adults $(n=1539 ; 72.6 \%$ male; $31.43 \pm 11.96$ years $)$ visiting a popular pornography portal completed the OPDQ, the Brief Symptom Inventory (BSI) and provided information on their OP use. Independent t-tests were conducted to compare users with an unproblematic and problematic use of OP. 
Of the users, $5.9 \%$ fulfilled the criteria for problematic use. This group consumed OP for longer amounts of time and showed higher levels of psychopathological distress (average $\mathrm{g}=1.12)$.

Overall, the results of the study indicate that problematic use of OP seems to be linked to severe psychological distress and thus might be a serious problem that demands clinical awareness.

\title{
P26
}

\section{Moderating Effect of family function in the relationship between temperament and smartphone addiction in adolescents}

\author{
Jung-Seok Choi ${ }^{1 *}$, Aruem Choi ${ }^{2}$, Ji Yoon Lee 3 , Sun Ju Chung ${ }^{3}$,Bomi Kim³, \\ Min Kyung Park ${ }^{3}$ \\ ${ }^{1} 1$ Department of Psychiatry, SMG-SNU Boramae Medical Center, Seoul, Republic of Korea 2 \\ Department of Psychiatry and Behavioral Science, Seoul National University College of Medicine, \\ Seoul, Republic of Korea \\ ${ }^{2}$ SMG-SNU Boramae Medical Center, Seoul, Republic of Korea \\ ${ }^{3}$ Department of Psychiatry, SMG-SNU Boramae Medical Center, Seoul, Republic of Korea \\ choiar90@gmail.com
}

Objectives: It is to confirm the moderating effect of family functioning on the relationship between temperament and smartphone addiction in adolescents.

Methods: Participants were 147 students (mean age $=14.42 \pm 1.344$ years; boys, $\mathrm{N}=$; girls, $\mathrm{N}=$ ). All subjects completed questionnaires including adolescent smartphone addiction scale, temperament and character inventory (JTIC), family ability and cohesion scale(FACESIII).

Results: With regard to temperament, novelty seeking and harm avoidance had positive correlations with severity of smartphone addiction. The interaction effect between novelty seeking temperament and family functioning was not significant, while the interaction effect between harm avoidance temperament and family functioning was significant. It showed a moderating effect on both family adaptability and family cohesion belonged to family functioning. This implies that the type of family adaptability and family cohesion interacts with the harm avoidance temperament level and thus has a different impact on smartphone addiction.

Conclusions: Our results suggest that adolescents with high harm avoidance temperament levels were found to be more likely to become addicted to smartphones if their family adaptability and family cohesion were close to extreme out of normal range.

Keywords: Smartphone addiction, family function, adolescent temperament 


\title{
P27
}

\section{Self-efficacy and clinical characteristics among excessive gaming users, casual gamers or non-gamers}

\author{
Jung-Seok Choi ${ }^{\text {** }}$, Sun Ju Chung' ${ }^{2}$, Ji Yoon Lee ${ }^{2}$,Aruem Choi², Bomi Kim², \\ Min Kyung Park ${ }^{2}$
}

${ }^{1} 1$ Department of Psychiatry, SMG-SNU Boramae Medical Center, Seoul, Republic of Korea 2

Department of Psychiatry and Behavioral Science, Seoul National University College of Medicine, Seoul, Republic of Korea

${ }^{2}$ Department of Psychiatry, SMG-SNU Boramae Medical Center, Seoul, Republic of Korea sunjujung1991@gmail.com

Objectives: The present study aimed to investigate the differences in self-efficacy depending on extent of gaming use. Furthermore, we investigated the clinical characteristics associated with game addiction in group comparisons.

Methods: A total of 158 young adults participated in this study: excessive gaming users diagnosed with internet gaming disorder (IGD) based on DSM-5, casual gamers those who play games regularly but not belong to IGD and non-gamers who do not any game among healthy controls. All participants completed self-reported questionnaires including Self-efficacy scale, Behavioral Activation / Inhibition System, Aggression questionnaire, Barratt Impulsiveness scale version 11, Beck Depression Inventory, Beck Anxiety Inventory, and Psychosocial Well-being Index.

Results: Analysis of covariance controlling for gender showed that levels of self-efficacy were statistically different from each group (IGD $<$ casual gamers $<$ non-gamers; $\mathrm{p}<.001$ ). Further, aggression $(\mathrm{p}<.001)$, impulsivity $(\mathrm{p}<.001)$, depression $(\mathrm{p}<.001)$, anxiety $(\mathrm{p}<$ $.001)$, stress $(\mathrm{p}<.001)$ and behavioral inhibition system $(\mathrm{p}<.001)$ were significantly higher in excessive gaming users compared with casual gamers and non-gamers.

Conclusions: These findings show that people who do more game use tend to have lower self-efficacy levels. Our study suggests self-efficacy may exert as a protective or risk factor in game users, particularly casual gamers those who at risk of developing excessive gaming users.

\section{P28}

\section{Neurophysiological reactivity to gaming related cues in Internet gaming disorder}

Jung-Seok Choi*, Bomi Kim, Jiyoon Lee, Minkyung Park, Aruem Choi, Sunju Chung

Department of Psychiatry, SMG-SNU Boramae Medical Center

dreamykim@gmail.com

Although the importance of Internet gaming disorder (IGD) has been growing, there is still a lack of understanding of them. IGD has deficits in emotional regulation and attentional bias toward online gaming-related cues. In this study, we investigated emotional intensity and at- 
tentional bias in IGD using the electrophysiological marker of late positive potential (LPP) that may reflect emotional and attentional processing.

A total of 63 subjects participated in this study. A series of game-related and neutral pictures were shown to 33 patients with IGD and 30 healthy control (HC) subjects while eventrelated potentials (ERPs) were recorded. To investigate the time-course of emotional intensity and attention to the stimuli, we divided into early and later LPP. Early LPPs were calculated as the mean value of amplitudes between 400 and $700 \mathrm{~ms}$ at the centro-parietal (CP3, $\mathrm{CP} 1, \mathrm{CPz}, \mathrm{CP} 2, \mathrm{CP} 4)$ and parietal (P3, P1, Pz, P2, P4) electrode sites, and later LPPs were calculated between 700-1000ms at the same electrode sites as the early LPP. At the centroparietal site, both early and later LPP amplitudes were observed higher for the game-related cues in the IGD group than in the HC group. At the parietal site, there were no significant results. Our results indicate that IGD is more emotional arousal than $\mathrm{HC}$, and their attention is biased to game-related cues

P29

\title{
Validating of Semi-structured Diagnostic interview for Internet Addiction scale (DIA) for clinical samples in Korean Children and adolescents
}

\author{
Sanyeowool Oh' ${ }^{1 *}$, YeongSeon Jo2, Soo-young Bhang ${ }^{3}$, Cheol-Soon Lee ${ }^{4}$, Yong-Sil Kweon \\ ${ }^{1}$ Department of Psychiatry, Nowon Eulji Hospital, Seoul \\ ${ }^{2}$ Department of Psychiatry, The Catholic University of Korea Uijeongbu St. Mary's Hospital, Seoul \\ ${ }^{3}$ Department of Psychiatry, Eulji University School of Medicine, Seoul \\ ${ }^{4}$ Department of Psychiatry, Gyeongsang National University School of Medicine, Gyeongsang \\ National University Changwon Hospital, Changwon \\ ${ }^{5}$ Department of Psychiatry, Uijeongbu St. Mary's Hospital, College of Medicine, The Catholic \\ University of Korea, Seoul \\ skykt27@naver.com
}

Background and aims: This study aimed to develop a semi-structured interview scale to measure internet / game / smartphone addiction. Inspired by the 9-item DSM-5 internet gaming disorder diagnostic criteria, we developed 10-item Diagnostic interview for Internet Addiction (DIA).

Methods: The subjects were students who were referred to as having internet addiction problem. Children aged 7 to 18 years were included in this study. DIA scale, Korean internet addiction scale (K, Young- scale), smartphone addiction scale (SAS-SV, S), Internet addiction Proneness scale questionnaire were conducted. Exploratory factor analysis and correlation analysis were performed to verify the validity of DIA. Based on results of exploratory analysis, Confirmatory factor analysis was performed utilizing Structural Equation Modeling in Amos 19.

Results: Results indicated that the DIA highly correlated with the scores of the K scale $(\mathrm{r}=.361, \mathrm{p}<.01)$, Young internet addiction test $(\mathrm{r}=.282, \mathrm{p}<.01)$, Internet Addiction Proneness Scale for Children $(\mathrm{r}=.555, \mathrm{p}<.01)$, Internet Addiction Proneness Scale for adolescents 
$(\mathrm{r}=.311, \mathrm{p}<.01)$. Factor analysis of the DIA revealed two factors. Results from the CFA indicated that the two-factor model demonstrated good model fit: TLI $=0.919, \mathrm{CFI}=0.950$, RMSEA $=0.058$.

Conclusions: The DIA scale appears to be a valid diagnostic scale for screening children and adolescents who are at risk of internet and smartphone addiction.

P30

\title{
Gender differences in the relationship between depression and internet gaming disorder
}

\author{
Dai-Jin Kim ${ }^{1 *}$, Hyun Cho ${ }^{2}$ \\ ${ }^{1}$ Department of Psychiatry, Seoul St. Mary's Hospital, The Catholic University of Korea, Seoul, Korea \\ ${ }^{2}$ Addiction Research Institute, Department of Psychiatry, Seoul St. Mary's Hospital, The Catholic \\ University of Korea, Seoul, Korea \\ sonap1@hanmail.net
}

Many studies have reported close links between depression and internet gaming disorder (IGD). And women were reported to have more symptoms of depression than men. If so, it could be assumed that the rate of female IGD is higher. However, the prevalence of IGD in women was lower than in men in most studies. The purpose of this study was to examine gender differences in the relationship between depression and IGD. Response style theory suggested that individuals had a consistent style that responds to depressed mood and that the level of depression of women was higher than that of men because of the difference of response style to depressed emotions. In this study, in women, it was assumed that the relationship between brooding and depression was stronger than the relationship between distraction and IGD. And for men, it was assumed that the result was the opposite. This assumption was verified by structural equation model. As a result of the analysis, both men and women had the strongest relationship between brooding and depression. In addition, the relationship between distraction and IGD was statistically significant only for women. Further research is needed on the relationship between depression and IGD. 
P31

Comorbidity of psychiatric disorders with internet addiction and their characteristics across the diagnosis in Republic of Korea child and Adolescent

\author{
Yong-Sil Kweon ${ }^{*}$, YeongSeon $\mathrm{Jo}^{2}$, Sanyeowool Oh${ }^{3}$, Soo-young Bhang ${ }^{4}$, Seung-Yup Lee ${ }^{5}$, \\ Marc N. Potenza ${ }^{6}$ \\ ${ }^{1}$ Department of Psychiatry, College of Medicine, Uijeongbu St. Mary's Hospital, The Catholic \\ University of Korea, Seoul, Republic of Korea \\ ${ }^{2}$ Department of Psychiatry, The Catholic University of Korea Uijeongbu St. Mary's Hospital, Seoul, \\ Republic of Korea \& Department of Psychology, The Sung-Shin women's University, Seoul, \\ Republic of Korea \\ ${ }^{3}$ Department of Psychiatry, Nowon Eulji Hospital, Seoul, Republic of Korea \\ ${ }^{4}$ Department of Child and Adolescent Psychiatry, Eulji University School of Medicine, Seoul, \\ Republic of Korea \\ ${ }^{5}$ Department of Psychiatry, College of Medicine, Uijeongbu St. Mary's Hospital, The Catholic \\ University of Korea, Seoul, Republic of \\ Korea \\ ${ }^{6}$ Departments of Psychiatry and Neuroscience, Child Study Center, The National Center on Addiction \\ and Substance Abuse (CASA Columbia), Yale University School of Medicine, New Haven, CT, USA \\ \& Connecticut Mental Health Center, New Haven, CT, USA \\ jo_oseon@naver.com
}

Background: Relationship between psychiatric comorbidity (ADHD, depression) and Internet Addiction (IA) have been reported. However little is known about the relationship between Autism Spectrum Disorder (ASD) and IA.

Methods: A multicenter clinical cohort study (c-CURE) was conducted in Seoul 20152018. We conducted semi-structured interviews (DIA, KSADSPL) and various self-reports. From base point data, 176 subjects were analyzed (male $=73 \%$, average age $=13.10$ ).

Results: Parental reports of children's internet usage was higher in the $\operatorname{ADHD}(34.3 \%)$ than in the non-ADHD group in children. On the other hand in the adolescents, the ASD group $(27.5 \%)$ showed higher internet usage than the non-ASD. Depression, anxiety, selfesteem, impulsivity showed statistically significant differences in adolescent depression and non-depression group. Interestingly the ASD group reported higher stress level on the Daily Stress questionnaire, family, friends, teachers, school subscales. But the depression group $(21.9 \%)$ only showed a difference in the academic subscales and low positive emotions on the happiness scale.

Conclusions: There were different patterns of IA and various psychosocial factors between ADHD, depression, ASD and non-symptom group. Therefore differentiated clinical intervention based on the developmental age and psychiatric disorders can be suggested. [This study was supported by a grant of the Korean Healthcare Technology R\&D Project, Ministry of Health and Welfare (HM14C2603).] 


\title{
P32
}

\section{Smartphone usage patterns of problematic smartphone user in China: A research based on a real- time monitoring Application (APK)}

\author{
Tie-Qiao Liu ${ }^{1},{ }^{2}$, Yue-Heng Liu ${ }^{1},{ }^{2}$, Jiang Long ${ }^{1},{ }^{2}$, Joël Billieux ${ }^{3}$, Yi-Feng Yuan ${ }^{4}$, \\ Man-Yun Li $i^{1},{ }^{2}$, Hang Zhang ${ }^{1},{ }^{2}$, Kai-Lin He ${ }^{1},{ }^{2}$, Hui-Qing Peng ${ }^{1},{ }^{2}$ \\ ${ }^{1}$ Department of Psychiatry, The Second Xiangya Hospital, Central South University, Changsha, \\ Hunan, China \\ ${ }^{2}$ National Clinical Research Center on Mental Disorders, Changsha, Hunan, China \\ ${ }^{3}$ Addictive and Compulsive Behaviours Lab, Institute for Health and Behaviour, University of \\ Luxembourg, Esch-sur-Alzette, Luxembourg \\ ${ }^{4}$ The Third Xiangya Hospital, Central South University, Changsha, Hunan, China \\ tiegezhongguo@126.com
}

Background: Problematic smartphone use has been increasingly drawing attention from international researchers. However, since most existing research is only based on self-report data, the actual usage patterns of smartphone users remain unclear. We developed an application (APP), identifying and recording real-time usage status of the smartphone users, to delineate the use of smartphone in Chinese undergraduates.

Methods: A random sample of 166 undergraduates were recruited. Their smartphone usage data has been recorded for 60 days by the App. Smartphone Addiction Proneness Scale (SAPS) was used to identify PSU.

Results: Their smartphone daily use time (duration) was $7.1 \pm 2.1 \mathrm{~h}$, and daily use count (frequency) was $118.0 \pm 61.8$ times. Classified by application varieties, the daily use time (duration) could be further divided into 10 categories as follows: Social (195min, 46\%), Lifestyle (56min, 13\%), Reading (53min, 13\%), Video \& Live broadcast (38min, 9\%), Games (30min, 7\%), Shopping (14min, 3\%), Education (12min, 3\%), Photography (9min, 2\%), Productivity ( $8 \mathrm{~min}, 2 \%$ ) and Music (7min, 2\%). In comparison to non-PSA group, participants from PSA group tended to spend more time on smartphone use, especially apps of Social, Reading, Video \& Live, Games, and shopping.

Conclusions: Undergraduates spend significant time on the smartphone. Moreover, Specific application categories usage contributes to discriminating problematic users, which are worth further researching. 


\title{
P33
}

\section{Peer support-based group intervention for excessive gamers}

\author{
Terhi Mustonen ${ }^{1 *}$, Risto Joensuu ${ }^{1}$, Helmi Korhonen ${ }^{1}$, Niko Mannikko², Paula Ojala ${ }^{3}$, \\ Heidi Ruotsalainen ${ }^{2}$, Maria Kaariainen ${ }^{3}$ \\ ${ }^{1}$ Sosped Foundation \\ ${ }^{2}$ Oulu University of Applied Sciences \\ ${ }^{3}$ University of Oulu \\ terhi.mustonen@sosped.fi
}

Limit'less Gaming Program provides a group-based intervention for young adults who play video games excessively but seek to reduce the role of gaming in their everyday lives. The program aims at preventing progression of problematic gaming behaviors and promoting gamers' overall well-being by strengthening their social skills, time management skills, and increasing physical activity. Peer support, instructor-guided thematic discussions, social group activities, and gamification of skill training are employed as the main methods. 25 young adults (18 to 27 years of age; one female) participated in pilot groups in five cities in Finland. Participants in each group attended ten weekly 3-hour meetings that were guided by a health care professional and a trained peer instructor with a history of problematic gaming. Measures of the participants' problematic gaming (i.e., Problematic Gaming Behavior Questionnaire) and psychological well-being (i.e., Warwick-Edinburgh Mental Well-Being Scale) were obtained prior to and following intervention, along with their self-reports on the time spent on gaming and other leisure activities, physical well-being (e.g., physical activity, eating habits, sleep), social well-being (e.g., social relations, feelings of loneliness), and the quality of life. Here we evaluate the effectiveness of Limit'less Gaming Program based on the results of the ongoing pilot research.

\section{P34}

\section{Social networking addiction and depressive symptoms among adolescents in Korea}

\author{
Jinhee Lee*, Seongho Min, Min-Hyuk Kim, Joung-Sook Ahn
}

Division of Child and Adolescent Psychiatry, Yonsei University Wonju College of Medicine, Wonju, Korea jinh.lee95@yonsei.ac.kr

Background: We aimed to investigate the association of social networking addiction and depressive symptoms among adolescent by analyzing data from the nationally representative sample of Korean adolescents aged 12-18 years.

Methods: Participants were categorized into one of the following three groups according to time spent on smartphone (less than $5 \mathrm{~h}$ and $5 \mathrm{~h}$ or more a day) and the main purpose of smartphone use (social networking purpose / other purposes; studying, games, entertain- 
ments etc.): moderate use group (MU), addictive use group with other purposes (AO), and addictive use group with social networking purpose (AS). We investigated the characteristics of groups and the risks of depressive symptoms using Chi-square analysis and multiple logistic regression analyses after adjusting for confounding factors.

Results: AO occurred in 3,634 (5.8\%) and AS occurred in 7,957 (12.8\%) of the 62,276 students. AS was more prevalent in older adolescents (16-18), persons reporting lower physical activity, lower sleep time, and more consumption of alcohol and tobacco. AS was associated with an increased likelihood of reporting depressive symptoms (OR 1.38, 95\% CI 1.16-1.38) compared with MU (OR 1.38, 95\% CI 1.30-1.46) and AO (Ref.).

Conclusions: Adolescents with social networking addiction have higher prevalence of depressive symptoms.

\title{
P35
}

\section{The Effect of Desire for Control on Smartphone Addiction: Mediating Effect of Modes of Control.}

\author{
Daijin Kim ${ }^{1 *}$, Dongjin Jung ${ }^{1}$, Hyun Cho ${ }^{2}$
}

${ }^{1}$ Seoul St. Mary's Hospital, The Catholic University of Korea

${ }^{2}$ The Catholic University of Korea

forever0851@gmail.com

Purpose: This study was designed to explore the effect of desire for control on Smartphone addiction, and to examine whether the effect of desire for control differs depending upon modes of control(positive assertive, positive yielding, negative assertive, negative yielding).

Methods: By online, participants $(\mathrm{N}=1,669)$ were asked to complete self-reports for evaluation, including desire for control (DC), modes of control (MC) scale of Shapiro Control Inventory and Smartphone Addiction scale (SA). Participants were consisted of 867 men and 802 women, and 20 s age group 834,30 s 835 people.

Results: The results showed that, DC had positive effect on SA. The effect of DC on SA varied according to $\mathrm{MC}$. Indirect effect of positive assertive mode and positive yielding mode was negative on SA significantly. However, indirect effect of negative assertive mode and negative yielding mode was positve.

Conclusions: Based on these results, Personal modes of control mediating Smartphone addiction. Implications and limitations of this study were discussed along with the suggestions for future research 
P36

\title{
Internet Behaviour and the threat of Internet Gaming Disorder for Medical Students in Indonesia
}

\author{
Karina Kalani Firdaus $^{1 *}$, Kristiana Siste Kurniasanti ${ }^{2}$ \\ ${ }^{1}$ Faculty of Medicine, Universitas Indonesia \\ ${ }^{2}$ Department of Psychiatry, Universitas Indonesia- Cipto Mangunkusumo Hospital \\ karinakalanif@hotmail.com
}

Students, especially young adults (YA), are at risk of addiction potentially effecting their studies, such as Internet Gaming Disorder (IGD). In India, $6 \%$ of medical students had severe disturbance due to internet gaming with an average gaming time (GT) of 7 hours with poor academic performance. Early detection is vital for prompt intervention since YA phase is critical for character development.

A cross sectional study of 211 medical students in Universitas Indonesia assessed the prevalence of IGD and internet behaviour using the Ten-Item Internet Gaming Disorder Test (IGDT-10) questionnaire in which addiction was diagnosed for score of 5 or more.

The IGD prevalence was 3\%, among those had an overall GT average of 6,12 hours and used the internet since childhood (under 10 years old). Among the gamers, casual games were most played (29,3\%), longest GT was during vacation (average 4,25 hours / day), using handphones / tablets $(83,8 \%)$, at home $(89,9 \%)$, and usually also played offline games $(75,8 \%)$. Students should be warned about IGD especially if gaming started since childhood. Since usage is mainly at home, parents should educate and remind children of their internet game play especially during vacation. Onset of internet usage and online gaming were significant factors $(\mathrm{p}<0.05)$.

P37

\section{Does social bond always protect you from Internet gaming disorder?}

\author{
Anise M. S. Wu ${ }^{l *}$, Shu Yu ${ }^{l}$, Jose Carlos Sanchez Prieto ${ }^{2}$, Meng Xuan Zhang ${ }^{l}$ \\ ${ }^{1}$ University of Macau \\ ${ }^{2}$ University of Salamanca \\ mogu.yus@gmail.com
}

Background: Previous studies consistently found social bond as a protective factor against Internet gaming disorder (IGD). This study investigated among Spanish young people whether any form of social bond was beneficial, even in the context of peers and parents with addiction.

Methods: Five hundred sixty-seven Spanish middle school and university students (Mage $=18.86$; range $=12$ to 31$)$ with Internet gaming experience voluntarily completed an anonymous questionnaire. IGD was measured by the Spanish version of the IGD-20 Test. 
Results: As hypothesized, IGD tendency was negatively correlated with social bond $(\mathrm{r}=-.40, \mathrm{p}<.001)$, and positively correlated with perceived peer and parent IGD ( $\mathrm{rs}=.14$ to $.45, \mathrm{p}<.01)$. Bootstrapping approach was used to test the hypothesized moderation model. Both peer and parent IGD weakened the protective effect of social bond, while having significant direct effects on IGD among females. Such moderating and direct effects of parent IGD were non-significant while peer IGD had significant direct effect among males. The total variance in IGD tendency explained was $21.1 \%$ and $29.0 \%$ respectively. Conclusions: Protective effect of social bond against IGD was observed but females were prone to influences from peer and parent's problem game use. Gender specific intervention programs may be called for.

\title{
P38
}

\section{Identification of genetic variants associated with Internet Gaming Disorder by targeted next generation sequencing}

\author{
Yeun-Jun Chung*, Minho Lee \\ The Catholic University of Korea, College of Medicine \\ minholee@catholic.ac.kr
}

Addictive use of the Internet and Internet-based games is not just a social phenomenon in countries with extensive Internet access infrastructure, but a potential psychiatric disorder termed Internet gaming disorder (IGD). Several previous studies have suggested a genetic background to IGD, however, genetic variants associated with IGD is still largely unknown. In this study, we aimed to identify the single nucleotide polymorphisms (SNPs) associated with IGD. For this, we designed a targeted gene panel for next-generation sequencing (NGS) analysis, which contains 150 addictive disorder associated genes. We performed the target NGS analysis with 40 IGD individuals and analyzed the IGD associated SNPs by comparing with 400 normal individual whole genome sequencing data. We identified 29 SNPs significantly associated with IGD. Of the 29 IGD associated SNPs, a number of addictive disorder related genes such as HTR3A, AUTS2, CHRNA7, GABRA5, and MYO5B were included. Our data can be helpful to understand the genetic background of IGD and may facilitate early intervention to help people at higher risk of IGD. 
P39

\title{
Internet gaming deteriorate children's daily living functioning at child psychiatry
}

\author{
Yuichi Takahashi ${ }^{1 *}$, Kumi Uehara Aoyama², Junichi Fujita ${ }^{3}$, Nao Toyohara $^{3}$, \\ Kyohei Yamamoto ${ }^{1}$, Runa Tanimoto ${ }^{1}$ \\ ${ }^{1}$ Yokohama City University Medical Center \\ ${ }^{2}$ Yokohama City University \\ ${ }^{3}$ Yokohama City University Hospital \\ kums@ballade.plala.or.jp
}

Daily life among children is not well illustrated. We conducted a survey on internet use behavior and daily living function using internet addiction test (IAT) and Questionnaire -Children with Difficulties (QCD) at two general hospitals in Yokohama city, Japan.

The survey was implemented to 694 first-visited children aged from 10 to 18 years old from April 2015 to March 2017. Children with no diagnosis, moderate to severe mental retardation and children who could not complete the questionnaire were excluded. Out of 418 patients, 190 patients who play games as main purpose of internet use were analyzed. 46 $(24.2 \%)$ of the patients mainly playing the game using internet corresponded to the problematic internet user group (IAT > 50) and about 15 (7.9\%) was the pathological internet user group (IAT > 70). Patients corresponding to problematic internet user group and the pathological internet user group, the deterioration of the daily living functioning in total score were seen in problematic users $(29.98 \pm 11.68)$ and pathological users $(26.87 \pm 11.15)$ compared with the normal internet user group $(33.31 \pm 11.78)(\mathrm{p}<0.05)$. From the results, patients with a tendency to addict on gaming using internet need support for their daily living.

\section{P40}

\section{Problematic internet use screening within well visits at primary care}

\author{
Nina Petricevic* \\ Teaching Institute of Public Health Dr. Andrija Stampar \\ nina.petricevic@stampar.hr
}

Aim: to test screening for Problematic internet use during regular students' well visit in school and adolescent medicine practice and to evaluate association with other risky health behaviour and mental health issues.

Subject and methods: 139 patients (96 females, mean age $=19.45, \mathrm{SD}=0.51$ ) who came in for regular, obligatory well visits for all 1st year university students. Data were collected from medical records and Problematic Internet Use Questionnaire Short-Form-9 (PIUQSF-9) was administered at examination. Patients were directly informed of test results and referred to more evaluation or treatment if needed. 
Results: All of the patients consented to fulfil PIUQ-SF-9, declaring it short and easy to use. Majority students were healthy, 17\% having chronic diseases, $24 \%$ are smokers, $68 \%$ had been drinking alcohol in the last month. The mean of the total score on the PIUQ-SF-9 was 19.28 ( $\mathrm{SD}=5.58)$. Lower self-esteem assessed by Rosenberg self-esteem scale $(\mathrm{p}=0.015)$ and alcohol use $(\mathrm{p}=0.002)$ was associated with higher scores on PIUQ.

Conclusions: With increasing problem of Internet addiction, we find it valuable to use PIUQ as a part of regularly scheduled well visits. Further evaluation is needed for selecting tests and appropriate patients' group.

\title{
P41
}

\section{High frequency users of visual sexual stimuli show a heightened effect in a reward motivated Pavlovian-to-Instrumental transfer paradigm}

\author{
Timothy J. Wells*, Martin Hula, Rachel R. Horsley \\ National Institute of Mental Health \\ timothy.wells@nudz.cz
}

Pavlovian-to-instrumental transfer (PIT) has been identified as a key mechanism involved in cue-triggered wanting in individuals that have an established compulsive behaviour in relation to a particular reward. Although mostly researched in relation to models of pharmacological dependency, the neurological basis of PIT has been linked to further compulsions and behavioural addictions. However, PIT has never been studied in relation to individuals exhibiting visual sexual stimuli (VSS) proclivities. We tested 80 male participants that reported using online VSS (40 low frequency; 40 high frequency) on a computerised PIT task involving monetary rewards. Data indicated that both Pavlovian and Instrumental learning was established. The results provide evidence of a heightened transfer effect in high frequency VSS users compared with low frequency users. Reward related cues lead to an increased likelihood of instrumental responses previously associated with the same reward. These findings indicate the probable similarities between the underlying mechanism involved in frequent VSS use and other compulsive behaviours. Moreover, the potential to trigger wanting highlights the dangers of unsolicited VSS online that may facilitate seeking behaviour or even relapse for individuals committed to abstinence. 


\title{
P42
}

\section{No evidence of impulsivity in high frequency users of visual sexual stimuli found using a go / no-go task}

\author{
Timothy J. Wells*, Martin Hula, Rachel R. Horsley \\ National Institute of Mental Health \\ timothy.wells@nudz.cz
}

\begin{abstract}
Abnormal displays of impulsivity have often been discussed in relation to addictive behaviours. The tendency to act without forethought is believed to be a predisposition that leads to risky behaviour and can exacerbate the development of compulsive behaviour. However, studies across different disorders including problematic use of visual sexual stimuli (VSS) use have produced mixed results. While there is some indication that impulsivity is related to symptom severity, other research found the effect of impulsivity to be much lower than anticipated. We tested 80 male participants that reported using online VSS (40 low frequency; 40 high frequency) on a computerised go / no-go task. As expected, the results of the task corresponded with a psychometric measure of impulsive action. However, we found no evidence that frequency of online visual sexual stimuli use was related either reaction times or inhibition of a pre-potent motor response. Research needs to focus on understanding the similarities and differences distinctive to the various behavioural addictions. The findings here suggest that high frequency online VSS users are not impaired in their ability to inhibit motor action. However, impulsivity is a multidimensional construct; it is possible that problematic use of online VSS may be more related to impulsive decision making.
\end{abstract}

\section{P43}

\section{The Use of Structured Instruments for Evaluating Cell Phone Addiction Severity}

\section{Octavian Vasiliu*}

Dr. Carol Davila University Emergency Military Central Hospital octavvasiliu@yahoo.com

Background and objective: Cell phone addiction is a very complicated behavioral addiction, because of the multiple uses associated to the smartphones nowadays, and also because a complete withdrawal from cell phone use is frequently impossible, or even dangerous from multiple objective reasons. In order to distinguish between normal and pathological cell phone use standardized instruments may be very helpful for both screening and monitoring purposes.

Methods: Data about validated instruments for the quantification of cell phone addiction severity were collected from the main electronic databases (PubMed, Cochrane, Embase, PsychInfo, Thomson Reuters / Web of Science). 
Results: The most studied instruments for quantification of cell phone addiction severity that have been found through this database search were Smartphone Addiction Proneness Scale- an instrument destined for adolescents, Smartphone Addiction Scale- a self-diagnostic scale, Smartphone Addiction Inventory- Short Form and Standard Form, Problematic Mobile Phone Use Questionnaire, Cellular Phone Dependency Questionnaire, and Brief Smartphone Addiction Scale- the last being a screening instrument for mobile phone addiction in school children.

Conclusions: A reasonably large number of questionnaires have been created for the measurement of the cell phone severity.

Their comparative validity has not been determined and their use for clinical purpose should be further investigated.

\title{
P44
}

\section{Mothers smartphone use and mother child connection}

Miriyam Farkash*

Ben Gurion University of the Negev

mirifaster@gmail.com

\section{P45}

\section{Generalized problematic Internet use and social anxiety and avoidance in Portuguese university students}

\author{
Berta Rodrigues Maia ${ }^{1 *}$, Helena TC Moreira², Maria Joao Soares ${ }^{3}$, Ana Telma Pereira ${ }^{3}$, \\ Antonio Ferreira de Macedo ${ }^{3}$ \\ ${ }^{1}$ The Catholic University of Portugal, Faculty of Philosophy and Social Sciences, Braga Regional \\ Centre; Centre for Philosophical and Humanistic Studies \\ ${ }^{2}$ Center for Research in Neuropsychology and Cognitive-Behavioral Intervention, Faculty of \\ Psychology and Educational Sciences, University of Coimbra, Coimbra, Portugal \\ ${ }^{3}$ Institute of Psychological Medicine, Faculty of Medicine University of Coimbra, Coimbra, Portugal \\ bmaia@braga.ucp.pt
}

Introduction: The unhealthy use of Internet has been associated with a number of negative outcomes and psychosocial problems.

Aim: To explore the gender differences, relations between variables and the predictive role of generalized problematic Internet use in distress / anxiety and avoidance in social situations.

Methods: 176 Portuguese university students, with a mean age of 20.13 years old (SD = 1,65; range: 18-25), filled a sociodemographic questionnaire, the Portuguese version of the Generalized Problematic Interne Use Scale 2 (GPIU) and the Social Interaction and Performance Anxiety and Avoidance Scale. 
Results: Males presented significant higher scores $(\mathrm{Md}=106.13)$ than females $(84.28)$ in GPIU Negative Consequences $(\mathrm{U}=1814.500, \mathrm{z}=-2,40, \mathrm{p}=.017)$, and females presented significant higher scores $(\mathrm{Md}=95.70 ; \mathrm{Md}=94.39)$ than males $(58.43 ; 63.90)$ in Distress / Anxiety and in Avoidance subscales $(\mathrm{U}=1391.500, \mathrm{z}=-3.83, \mathrm{p}=<.001 ; \mathrm{U}=1577.500, \mathrm{z}=$ $-3.136, \mathrm{p}=.002)$. The Distress / Anxiety subscale was significantly and positively correlated with all GPIU factors. Preference for Online Social Interaction, Deficient Self-Regulation, and gender were significant predictors of Distress / Anxiety in social situations. Conclusions: This study confirms the harmful role of generalized problematic Internet use in social interactions.

\title{
P46
}

\section{A Study of Psychological Characteristics upon Problem Stock Trading among Online Stock Investors}

\author{
TaeKyung Lee ${ }^{1 *}$, Joohyun Han², Jong Huk Choi ${ }^{1}$, Sungwon Roh ${ }^{3}$ \\ ${ }^{1}$ ChunCheon National Hospital \\ ${ }^{2}$ National Center for Mental Health \\ ${ }^{3}$ Hanyang University Hospital \\ TKLeeMD@gmail.com
}

Backgrounds: Online stock trading contributes to reduced transaction times and costs. However, these benefits may lead to trade in an excessive manner. This study explores the manifestations of Problem Online Stock Trading (POST) and the links with other psychological problems via the perceptions of online stock traders.

Methods: We recruited the participants aged between 19 and 65 years among the online panel of online research service. The sample size of the online panel survey was 500 . Of these 500 participants, $50.8 \%(\mathrm{~N}=254)$ were men and $49.2 \%(\mathrm{~N}=246)$ were women. We compared POST group to normal group with demographic characteristics and psychological measurements such as depression, anxiety, impulsiveness, and thought rumination.

Results: Sixty-seven (13.4\%) of the participants were placed into POST group. There was no difference in demographic characteristics. However, POST group showed higher psychological scale scores than a normal group such as depression, anxiety, impulsiveness, and thought rumination.

Conclusions: The prevalence of POST turned out a relatively frequent phenomenon among the online stock investors. This study suggests that POST should be considered as an important public health problem that deserves the attention of the scientific community. 


\title{
P47
}

\section{Neurobiological mechanisms of time discount disability in psychiatric disorders: a systematic review of magnetic resonance imaging studies}

\author{
Yoshihiro Noda*, Muhammad AbdElMoneam ElSalhy, Takahiro Miyazaki, \\ Shinichiro Nakajima, Fumi Masuda, Kamiyu Ogyu, Masataka Wada, Masaru Mimura \\ Department of Neuropsychiatry, Keio University School of Medicine \\ mu_salhy@yahoo.co.jp
}

Background: Time discount (TD) refers to the discount of the reward value with delay when comparing a certain reward with the current reward value. TD was increased with impulsivity in patients with attention deficit / hyperactivity disorder (ADHD) or behavioral and substance addiction, whereas TD was decreased in patients with anorexia nervosa. Here, mainly two neural networks associated with the actions of short-term interests and long-term benefits are identified in the TD process. However, the detailed neurobiological mechanisms of TD still remain unelucidated in these populations.

Methods: A systematic review was conducted following the PRISMA statement

Results: Out of 503 records, 31 studies were identified, all of which employed a magnetic resonance imaging. There were two neurobiological networks associated with TD: one is the neural network implicated in evaluating the value of the reward while the other is the network linked to engagement of prospective thought and future planning. Brain regions involved during TD task were the prefrontal cortex, insular cortex, ventral striatum, cingulate cortex, parietal cortex, and midbrain.

Conclusions: This review generated consistent findings in the neural basis of TD in patients with psychiatric disorders. Such neurobiological differences of TD may represent the potential pathophysiology of these psychiatric disorders. 


\title{
P48
}

\section{Generalized problematic Internet use and attachment styles in Portuguese university students}

\author{
Berta Rodrigues Maia ${ }^{1 *}$, Helena TC Moreira ${ }^{2}$, Ana T Pereira ${ }^{3}$, Maria Joao Soares $^{3}$, \\ Antonio F. Macedo \\ ${ }^{1}$ The Catholic University of Portugal, Faculty of Philosophy and Social Sciences, Braga Regional \\ Centre; Centre for Philosophical and \\ Humanistic Studies \\ ${ }^{2}$ Center for Research in Neuropsychology and Cognitive-Behavioral Intervention, Faculty of \\ Psychology and Educational Sciences, \\ University of Coimbra, Portugal \\ ${ }^{3}$ Institute of Psychological Medicine, Faculty of Medicine University of Coimbra, Portugal \\ bmaia@braga.ucp.pt
}

Introduction: Some recent studies have found problematic Internet use to be related to attachment styles.

Aim: To explore the relations between generalized problematic Internet use (GPIU) risk levels and general attachment-related anxiety and avoidance.

Methods: A sample of 300 Portuguese university students, with a mean age of 20.37 years old ( $\mathrm{SD}=1,72$; range: $18-25)$ filled the Portuguese versions of the Generalized Problematic Interne Use Scale 2 and the Experiences in Close Relationships- Relationship Structures Questionnaire.

Results: $56.9 \%(\mathrm{n}=160)$ of participants showed 'low risk' of GPIU, 37\% $(\mathrm{n}=104)$ 'medium risk' and $6 \%(\mathrm{n}=17)$ 'high risk'. Levels of attachment-related avoidance $(\mathrm{M}=20.46$, $\mathrm{SD}=5.63)$ were higher than levels of attachment-related anxiety $(\mathrm{M}=11.34, \mathrm{SD}=4.59)$. A Kruskal-Walis Test revealed a statistically significant difference in attachment-related anxiety levels across three different risk GPIU groups (Gp1, $n=157$ : low risk, Gp2, $n=101$, medium risk, Gp3, $\mathrm{n}=17$ : high risk), X2 $(2, \mathrm{n}=275)=21.64, \mathrm{p}=<.001$. The GPIU high-risk group recorded a higher median score $(\mathrm{Md}=190.24)$, than medium $(\mathrm{Md}=157.26)$ and low $(\mathrm{Md}=119.96)$ risk groups.

Conclusions: Future longitudinal studies are needed to clarify if attachment-relation anxiety is a possible distal precursor of GPIU. 
P49

\title{
Research on factors of Smartphone overdependence management application usage between smartphone overdependence group and normal group
}

\author{
In Young Choi ${ }^{*}$, MunJoo Choi ${ }^{1}$, Dai-Jin Kim² \\ ${ }^{1}$ Department of Medical Informatics, College of Medicine, The Catholic University of Korea, Seoul, \\ Republic of Korea 2. Department of Biomedicine \& Health Sciences, College of Medicine, \\ The Catholic University of Korea, Seoul, Republic of Korea \\ ${ }^{2}$ Department of Psychiatry, Seoul St. Mary's Hospital, College of Medicine, \\ The Catholic University of Korea, Seoul, Republic of Korea \\ cmj7705@naver.com
}

As people get close to smartphones, problems are emerging. Problematic smartphone usage lead social problems and daily life disorder. Therefore, necessity of proper smartphone use is emerging. For that, needed of self-control management service are emerged, we developed a smartphone application to deliver smartphone overuse prevention. In this study, we aim for investigating the acceptance of smartphone application which we developed, between smartphone overdependence group and normal group.

The research model theory was based on Technology Acceptance Model of Acceptance and Use of Technology, which were modified to be applied to the case of smartphone overuse. We conducted 500 smartphone users through online surveys from the age of 19 to age 60 in South Korea, 2018. The participants were randomly selected. We use Smartphone Addiction Proneness Scale to measure participant's smartphone dependency. Groups are divided into two, smartphone overdependence group and normal group. Structural Equation Model analysis was conducted using Amos ver. 24, to evaluate between two groups and variables to test hypothesis under $95 \%$ confidence interval.

Research results are expected to provide critical factors of Smartphone overdependence management application usage between smartphone overdependence group and normal group to consider when implementing in future systems and it will be the solutions that tackle the issue of smartphone overuse.

\section{P50}

\section{Psychometric Development of the Problematic Stock Trading Scale}

\author{
Sungwon Roh ${ }^{l^{*}}$, Tae Kyung Lee ${ }^{2}$, Joohyun Han ${ }^{3}$, Euihyeon $\mathrm{Na}^{4}$ \\ ${ }^{1}$ Hanyang University \\ ${ }^{2}$ Chuncheon National Hospital \\ ${ }^{3}$ National Center for Mental Health \\ ${ }^{4}$ Incheon Chamsarang Hospital swroh@hanyang.ac.kr
}

Background and Aims: Despite the increased number of retail investors and growing concern over their addictive behavior over the past few decades, there are no reliable and valid instruments for assessing problematic stock trading. The aim of this study is to develop the 
Problematic Stock Trading Scale (PSTS), a valid and reliable scale that measures problematic stock trading.

Methods: A sample of 500 retail investors was used in this study. Twenty-two items (nearly twice the final number of items) were initially selected as preliminary items, based on previous addiction scales such as gambling and substance use disorders as well as the clinical experience of the experts involved. The final items on the PSTS were determined through an exploratory factor analysis.

Results: Three core factors related to problematic stock trading were revealed from the exploratory factor analysis. The 12-item PSTS consists of five 'Preoccupation' factor items, four 'Risky Investment' factor items, and three 'Cognitive Distortion' factor items. Reliability and external validity were confirmed.

Conclusions: The present findings highlight the potential use of the PSTS for future research and possibly for clinical application by defining problematic stock trading as a behavioral addiction.

\title{
P51
}

\section{Method of Biological Feedback in The Prevention of The Use of Psychoactive Substances of Teenagers}

\author{
Sveta Berdibayeva ${ }^{1 *}$, Gaukhar Aldambergenova², Zhanylsyn Sabirova ${ }^{3}$, \\ Assiya Kukubayeva ${ }^{4}$, Marta Abdykalikova ${ }^{5}$, Satybaldy Berdibayev ${ }^{6}$ \\ ${ }^{1}$ Al-Farabi Kazakh National University \\ ${ }^{2}$ Kazakh National Women's Teacher Training University \\ ${ }^{3} \mathrm{Kh}$. Dosmukhamedov Atyrau State University \\ ${ }^{4}$ A. Myrzakhmetov Kokchetau University \\ ${ }^{5}$ Academy of Public Administration under the President of the Republic of Kazakhstan \\ ${ }^{6}$ L.N.Gumilyov Eurasian National University \\ berdibayeva.sveta1@mail.ru
}

The article is devoted to analyses of modern holistic educational environment as synergetic potential resource to increase the effectiveness of biofeedback in order to prevent of drug abuse among students. The advantages of biofeedback in comparison with other methods of self-regulation are discussed. The phases of biofeedback process described as interconnected components of a single process that is possible by hardware and software holistic educational environment. There are connection between different biofeedback instruments and psychophysiological specific training conditions. Research allows determining the effectiveness of work by biofeedback among teenagers. A questionnaire "My regulation of behaviour" (V.I. Morosanova) and a questionnaire determining the teenagers' attitude to drugs is used in order to develop samples. The results of forming experiment have revealed statistically significant increase in the level of adolescents' self-control in experimental group after class with biofeedback. Changing attitudes to drugs occurred only in the experimental group of adolescents who participated in the forming experiment. The results provide that systematic synergetic approach to prevent of drug dependency. The study received practical confirmation of the 
idea that the state of psychophysiological mechanisms with individual involvement in substance use can be objectively evaluated and managed by psychophysiological training to improve of self-regulation effectiveness.

P52

\title{
Addictive Showing Photos or Videos on Social Media for More Praise? A Tourism Psychological Survey in Chinese College Students
}

\author{
Fengjun Cui ${ }^{1 *}$, Jiliang Tang ${ }^{2}$, Jiayuan Yu ${ }^{3}$ Lida Dong ${ }^{1}$ \\ ${ }^{1}$ Taizhou University, Taizhou(Zhejiang), China \\ ${ }^{2}$ Taizhou University, Taizhou(Zhejiang), China; Inner Mongolia Normal University, Hohhot, China \\ ${ }^{3}$ Nanjing Normal University, Nanjing, China \\ 394908693@qq.com
}

More and more young people like to show self-shooting photos or videos during daily life on the kinds of social media, some of them even are addicted to do that. In recent years, Researches have been raised regarding the addictive use of social media. This study mainly plan to examine the relation between evaluation by others and showing tourism photos or videos on social media in sample of 1407 Chinese college students (Mean age $=19.45$ years, range $=17$-23years) who completed the research-designed questionnaire. The research has found, nearly $41.86 \%$ of all students concern the tourism photos or videos on self-social media whether or not can catch people eyes when they plan to shoot photos or videos, nearly $55.58 \%$ of all students understand and support this showing behavior; Nearly $74.98 \%$ of all students hope their tourism photos or videos can acquire more others, positive evaluation on the self-social media, and nearly $35.75 \%$ of all students may feel very disappointed if few people give them praise (more females feel very disappointed than males significantly, $\chi^{2}=$ 11.078, $\mathrm{P}<0.01)$.

Keywords: Addictive Showing Photos or Videos; Social Media; Evaluation; Tourism Psychological Survey; Chinese College Students 


\title{
P53
}

\section{Determinants of prescription opioid craving and opioid-related hedonic feelings among patients with chronic pain prescribed opioid therapy}

\author{
Leah Frimerman ${ }^{1 *}$, Marc O. Martel $^{2}$ \\ ${ }^{1}$ First Author \\ ${ }^{2}$ McGill University \\ leah.frimerman@mail.mcgill.ca
}

There has been a sharp rise with opioid misuse and addiction in patients with pain. Research has shown that opioid craving is a robust determinant of prescription opioid misuse. There is reason to believe that hedonic feelings associated with opioid use (e.g., pleasure, satisfaction) could also contribute to opioid misuse. However, this has yet to be examined in patients with pain. An objective of this study was to examine the association between opioidrelated hedonic feelings and prescription opioid misuse in patients with chronic pain. We also examined the factors contributing to opioid craving and opioid-related hedonic feelings. In this 14-day longitudinal diary study, patients $(\mathrm{n}=38)$ completed daily diaries assessing pain, psychological, and opioid-related variables. Prescription opioid misuse was also assessed. Multilevel analyses revealed that daily levels of pain intensity, negative affect, and catastrophizing were significantly associated with opioid craving. Results indicated that pain intensity, but not psychological factors, were associated with opioid-related hedonic feelings. Elevated reports of opioid craving and opioid-related hedonic feelings were associated with more frequent opioid misuse behaviors, such as taking more opioids than prescribed. Our findings suggest that opioid craving and opioid-related hedonic feelings may contribute to prescription opioid misuse in patients with chronic pain.

P54

\section{Effects of Cognitive-Behavioral Therapy with mindfulness on kleptomania}

\author{
Yuka Asami $^{1 *}$, Kazutaka Nomura ${ }^{2}$,Hironori Shimada ${ }^{2}$, Hiroyo Ohishi ${ }^{3}$, Masayuki Ohishi $^{3}$ \\ ${ }^{1}$ School of Human Sciences, Waseda University \\ ${ }^{2}$ Waseda University \\ ${ }^{3}$ Ohishi Clinic \\ yuka.asami@akane.waseda.jp
}

In Japan, the recidivism rate for theft is high, some of which may be caused by kleptomania. Increased treatment for kleptomania has been demanded to improve preventive measures to reduce thefts. Moreover, it is presumed that stealing in kleptomania is easily caused by automatic response to emotional arousal based on self-imposed rules. Therefore, the purpose of this study was to examine the effectiveness of group Cognitive-Behavioral Therapy (CBT) including mindfulness for kleptomania. The sample included 8 kleptomania patients who at- 
tended the CBT program. We tested the extent of adherence to self- imposed rules and severity of kleptomania twice (before and after the program).

Regarding the severity of kleptomania, scores on Kleptomania Symptom Assessment Scale significantly decreased $(\mathrm{t}(7)=6.98, \mathrm{p}<.01)$ after the program. Score on Distress Tolerance Scale as extent of adherence to self-imposed rules significantly decreased $(t(7)=3.01, p$ $<.05$ ). Additionally, regarding quality of life (QOL), scores on SRS-18 as a measure of shortterm QOL significantly decreased $(\mathrm{t}(7)=2.82, \mathrm{p}<.05)$, but those on GHQ28 and SDISS as a measure of long-term QOL did not. Improvement of distress tolerance suggested that CBT with mindfulness is effective for reducing adherence to self-imposed rules. Moreover, it is assumed that improvement of long-term QOL was necessary to directly treat complications in kleptomania for improving daily distress.

\title{
P55
}

\section{Exploring the Test of Mobile Phone Dependence psychometric properties in Portuguese Population}

\author{
Paulo Dias ${ }^{1 *}$, Paulo César Dias ${ }^{2}$, Mariano Choliz ${ }^{3}$ \\ ${ }^{1}$ Catholic University of Portugal \\ ${ }^{2}$ Universidade Católica Portuguesa \\ ${ }^{3}$ Universidad de Valencia \\ pcdias@braga.ucp.pt
}

Mobile phones are indispensable in our daily life. Although their original function was to allow people to connect with each other, currently they allow us to have access to information, to involve in social networks, to pay services, to play games, etc. Additionally, research has suggested that most of the users tend to make an appropriate use of this technology, but many of them present an excessive use. In this paper, we investigate the psychometric properties of the Test of Mobile Phone Dependence, a measure developed by Chóliz (2012), in accordance with the dependence criteria of the Diagnostic and Statistical Manual for Mental DisordersFourth Edition-Text Revised (DSM-IV-TR; American Psychiatric Association, 2000). Data was collected in a sample of 907 Portuguese adolescents and adults, aged 12 to 25 years old. Results of the exploratory factor analysis, with promax rotation, suggest an adequate structure of the measure with a four factor structure - abstinence, excessive or problematic use, consequences of mobile use, tolerance and lack of control - with loadings higher than .4. Reliability of the measure was high, with Cronbach alfas ranging between .754 and .888 . Gender differences were found. These data allow us to recommend TDM use in Portuguese population. 
P56

Defining essential elements of recovery from alcoholism: The experiential perspective-Comparison of the recovery elements between Japan and the US -

\author{
Yoshiaki Kindaichi", Misuzu Saitou, Minako Okabe, Arina Kasai, Sigeki Yamazaki \\ Hakuhou Clinic \\ hakuhou.kindaichi@gmail.com
}

Background: Aspects of alcohol recovery in the US were extensively studied by Kaskutas (2014) and Brockman (2016). We studied such aspects in Japan, and the results were compared with those in the US. The purpose of this research was to define the essential elements of recovery from alcoholism that could be common among people with different cultural backgrounds.

Methods: Using multidimensional approach, (a)meta-analysis of prior research surveys on the alcohol recovery, and free- descriptive questionnaires for both (b) alcoholism patients and (c) alcoholism professionals in Japan were integrated with varying dimensionality to define various aspects of the recovery. Then, the results were compared with those in the US using meta-analytic integration, to find their similarities and differences.

Results and Discussion: As the common elements, 6 categories were extracted: "Maintaining abstinence from alcohol", "physical health recovery", "mental health recovery", "behavioral recovery", "recovery of interpersonal relationships" and "social recovery". The main difference was the Spiritual Factor, which suggests that while Americans set spirituality as a transcendental existence, Japanese characteristically set it as gratitude to more familiar objects, including nature, others and animals.

P57

\title{
Food Addiction in Medical Student at Atma Jaya Catholic University of Indonesia: Any Impact on Obesity?
}

\author{
Eva Suryani ${ }^{1^{*}}$, Nicholas Hardi ${ }^{2}$, Ria Yunita $R^{3}$
}
${ }^{1}$ Department Psychiatry and Behavioral Science, School of Medicine and Health Science, Atma Jaya Catholic University of Indonesia
${ }^{2}$ Resident, Department of Psychiatry Faculty of Medicine Universitas Indonesia, Indonesia
${ }^{3}$ Resident, Department of Psychiatry, Faculty of Medicine, Public Health and Nursing Universitas
Gadjah Mada, Yogyakarta
eva.suryani@atmajaya.ac.id

Introduction: Obesity has become a global burden of disease, including Indonesia. High calories intake is one of its causes. People nowadays tend to consume hyperpalatable foods, which are highly addictive. 
Methods: The study design was cross sectional and involved Atma Jaya Medical School's preclinic medical student $(n=471)$ as samples. Research variables were assessed by Yale Food Addiction Scale (YFAS), Binge Eating Scale (BES) and Mini ICD 10.

Results: We found that food addiction prevalence was 5.2\%. Some of them were comorbid with binge eating disorder (1.6\%) and both binge eating disorder and bullimia nervosa $(0.4 \%)$. No statistically significant correlation were identified between food addiction and obesity $(\mathrm{p}=0.769)$.

Conclusions: As previous researches revealed, food addiction and obesity didn't have a consistent result. Various factors need to be reconsidered for a better result to show food addiction roles toward obesity.

Keywords: food addiction, obesity, binge eating disorder, bullimia nervosa, YFAS

\title{
P58
}

\section{Using the Syndrome Model of Addiction: a Preliminary Consideration of Psychological Stats and Traits}

\author{
Elda Mei Lo Chan ${ }^{1}$, $^{*}$, Ryan Hing Yan Wong', Matthew A. Tom ${ }^{3}$, Rhiannon Chou Wiley ${ }^{4}$, \\ Howard J. Shaffer ${ }^{3}$ \\ ${ }^{1}$ St. John's Cathedral Counselling Service \\ ${ }^{2}$ Tung Wah Group of Hospitals Integrated Centre on Addiction Prevention and Treatment \\ ${ }^{3}$ Division on Addiction, The Cambridge Health Alliance; Harvard Medical School \\ ${ }^{4}$ Division on Addiction, The Cambridge Health Alliance \\ elda.chan@sjcshk.com
}

This poster describes a collaborative research project between the Division on Addiction, Cambridge Health Alliance, a Harvard Medical School Teaching Hospital, and the Integrated Centre on Addiction Prevention and Treatment (ICAPT) of the Tung Wah Group of Hospitals (TWGHs) in Hong Kong. The goal of this research is to better understand the syndrome model of addiction by establishing an epidemiology of addiction treatment seekers across various expressions of addiction (e.g., alcohol and other psychoactive drugs, gambling, sexual behaviors, online gaming). This poster presents initial findings about the demographic and psychological characteristics of three groups of treatment seekers in TWGHs ICAPT: (1) treatment seekers for chemical expressions of addiction $(\mathrm{n}=75)$; (2) treatment seekers for behavioral expressions of addiction $(\mathrm{n}=268)$; and (3) a comparison group seeking services $(\mathrm{n}=18)$ unrelated to addiction. The initial findings identified various common psychological vulnerabilities (i.e., trait anxiety, state anxiety, depression, using emotional support and venting as psychological coping, higher levels of attentional impulsiveness) shared among both the behavioral expression and chemical expression groups, and possible unique psychological characteristics associated with each expression of addiction (e.g., coping mechanisms). These findings provide support for conceptualizing addiction with behavioural and chemical expressions as a syndrome. 


\title{
P59
}

\section{Evaluating the efficacy of Expressive Arts- based intervention on Rehabilitation for people with Gambling problem in Hong Kong: A Randomized- controlled Trail}

\author{
Mandy S.M. WU ${ }^{1^{*}}$, Rainbow T.H. $H o^{2}$ \\ ${ }^{1}$ Tung Wah Group of Hospitals Even Centre \\ ${ }^{2}$ The Centre on Behavioural Health, University of Hong Kong \\ mandy.wu@tungwah.org.hk
}

Tung Wah Group of Hospitals Even Centre initiated a project utilizing Expressive Arts-based Intervention on rehabilitation for people with gambling problems. This study aims to explore the efficacy of an Expressive Arts-based programme in reducing gambling desires and actual behaviours, enhancing gambling-related self-efficacy, self-control, and managing stress among people with gambling problem.

This study adopted a mixed-method, non-blind, 2-arm randomized controlled study, relying on both quantitative and qualitative data, collected from self-administered assessments at 3 separate time points and focus group interviews respectively. 82 adults with symptoms of gambling disorder were invited to join the Expressive Arts-based intervention programme and this evaluation study, and 68 effective sets of data were garnered for analysis. By comparing with control group, according to Bonferroni corrected post-hoc tests, the intervention group showed significantly greater improvement in self-control, lower level of gamblinginduced stress and cravings-related distress at T2 (3 months after intervention), lower in problem gambling severity at T1 (post-intervention), as well as a higher level of confidence in abstinence of gambling activities at both $\mathrm{T} 1$ and $\mathrm{T} 2$. Interviewees reflected that awareness of emotions, emotion expressions and broadened perspective towards gambling issues in the Expressive Arts-based intervention programme were helpful to restrain them from gambling. 


\title{
P60
}

\section{A content analysis of gambling operators' Twitter accounts at the start of English Premier League football season}

\author{
Elizabeth Alice Killick*, Mark D Griffiths \\ Nottingham Trent University \\ elizabeth.killick2017@my.ntu.ac.uk
}

The use of social media is now an established strategy to engage and maintain customer loyalty. The purpose of the present study was to examine the Twitter accounts of ten of the largest online sports betting operators in the UK to determine what marketing strategies were employed. More specifically, this study analyzed 3,375 tweets posted by the operators during the opening weekend of the 2018-2019 English Premier League football season using a content analysis methodology. The results demonstrated that multiple strategies were used including hashtags to link gambling operator tweets with major sporting events, and the use of numerous promotional campaigns. Notably, over $90 \%$ of the tweets contained no responsible gambling information. The quantity and content of social media posts underline the need for a review of the current advertising regulations in the UK. Further research should examine how exposure to sports betting social media marketing influences gambling behavior. 


\title{
TRAINING SEMINAR ON BEHAVIORAL ADDICTIONS
}

\section{TS-1}

\section{The concept and diagnosis of behavioral addiction}

\author{
John B. Saunders* \\ Centre for Youth Substance Abuse Research, University of Queensland, and the Faculty of Medicine, \\ University of Sydney \\ mail@jbsaunders.net
}

The concept of behavioral addiction has been applied to several repetitive human behaviors, and there is much debate about this concept and which behaviors and activities may become addictive. Mostly, it applies to intense repetitive behaviors which have the effect of immersing the individual involved. The two broadly accepted behavioural addictions involve gaming and gambling, especially in their electronic and / or online forms. This lecture will describe the features of online (Internet) gaming disorder as an exemplar of a behavioural addiction. While the accessibility and power of the Internet has transformed our lives and much is for the good, there are negative aspects and one of these is the development of addiction to online gaming. Numerous online games have been developed, with currently the most popular genre being the "battle royale" type, and those games which are the most engaging are also the most immersive and addictive. The central characteristics of Online / Internet Gaming Disorder, which have been incorporated into the International Diagnostic Systems of ICD-11 and DSM-5, will be described. In ICD-11 it is defined as (i) impaired control over the onset, duration and termination of gaming, (ii) increasing priority of gaming in the individual's life, with it taking precedence over other interests and responsibilities, and (iii) continuation or escalation of gaming despite negative consequences. The diagnosis also requires significant impairment in personal, family, social, education, occupational or other important areas of functioning. This presentation will review the current evidence for the addictive nature of gaming disorder and will describe some practical approaches to diagnosis. For other repetitive human behaviors to be considered addictive, there must be evidence of an "addictive drive" but also evidence of impairment in other aspects of life and / or harm to health and well-being. A distinction must be made between enjoyable recreational activities and those that develop into harmful disorders. 


\title{
TS-2
}

\section{What is happening in the brains of individuals with behavioral addictions and how is this related to treatment and recovery?}

\author{
Marc N. Potenza1,2,3* \\ ${ }^{1}$ Yale Center of Excellence in Gambling Research \\ ${ }^{2}$ Women and Addictions Core of Women's Health Research at Yale \\ ${ }^{3}$ Neuroscience and Child Study Yale University School of Medicine \\ marc.potenza@yale.edu
}

The disorders that constitute behavioral addictions (or disorders due to addictive behaviors, as termed in the eleventh edition of the International Classification of Diseases - ICD-11) include gambling disorder and gaming disorder, with other possible conditions relating to other behaviors (e.g., pornography viewing, buying and shopping, and social networking). In considering the definitions and classifications of these conditions, an understanding of the brain biology is important. Further, such an understanding may provide important information into the etiologies of the conditions, their emergence and their treatments or natural recoveries. In this presentation, data will be presented on the neurobiologies of behavioral addictions and how brain changes may relate to the emergence of these disorders and recoveries from the conditions. Implications for treatment development will also be discussed.

\section{TS-3}

\section{Cognitive-behavioral therapy in the treatment of behavioral addictions - evidence from research and clinical practice}

\author{
Kai W. Müller* \\ Outpatient Clinic for Behavioral Addictions, University Medical Center Mainz, Germany \\ muellka@uni-mainz.de
}

Gambling Disorder and Internet Gaming Disorder are new psychiatric phenomena affecting the lives of patients in a detrimental way. Both disorders are currently being perceived as non-substance related addiction disorders (behavioral addictions). Those are characterized by the evolution of symptoms typical for substance dependence in spite of the absence of drug intake. Core symptoms include preoccupation with the behavior, tolerance, withdrawal and continued use despite negative consequences in different areas of life. Research has shown that behavioral addictions are associated with suffering, heightened psychopathological symptoms, increased rates of comorbid disorders and decreases in psychological wellbeing. This emphasizes the need for sound treatment programs.

Clinical research and practice in the treatment of Gambling Disorder has shown that in particular cognitive-behavioral approaches (CBT) are effective. There is also some evidence that a combination of CBT and psychotropic medication are enhancing treatment outcomes. Yet, the rate of relapses after treatment among patients is still too high that one could speak of a satisfying therapeutic solution. Current research has demonstrated that implementation 
of supportive elements (e.g. computer assisted cognitive bias modification, strategies derived from third wave CBT) might be promising additions to the classical CBT-approaches.

Regarding Internet Gaming Disorder there is a clear lack of controlled clinical studies on treatment efficacy. Yet, first results have emphasized that also here, CBT is a promising type of intervention. The main aim of CBT is to identify and modify cognitive distortions and to enable patients to shift activities towards alternative activities.

The lecture will provide the audience with relevant information on the clinical aspects of Gambling Disorder and Internet Gaming Disorder and will derive CBT-elements appropriate to therapeutically address the main issues of both disorders. For Internet Gaming Disorder, a manualized CBT-treatment program (STICA) is introduced. Most recently, STICA has been successfully evaluated in a multicenter randomized clinical trial and showed good response rates and a high fidelity in modifying the problem behavior.

\section{TS-4}

\section{Pharmacotherapy of behavioral addictions}

\section{Jon Grant ${ }^{*}$}

Department of Psychiatry, University of Chicago, USA

jgrant4@bsd.uchicago.edu

Several behaviors, besides psychoactive substance ingestion, produce short-term reward that may engender persistent behavior despite knowledge of adverse consequences, i.e., diminished control over the behavior. Diminished control is a core defining concept of psychoactive substance dependence or addiction. This similarity has given rise to the concept of nonsubstance or "behavioral" addictions, i.e., syndromes analogous to substance addiction, but with a behavioral focus other than ingestion of a psychoactive substance. Which behaviors to include as behavioral addictions is still open for debate. This talk will discuss the pharmacotherapy of behavioral addictions and targets for pharmacotherapy.

Learning Objectives

1. Participants should develop an understanding for the evidence-base for pharmacotherapy of behavioral addictions;

2. They should be able to understand the neurobiology and cognitive aspects of behavioral addictions and possible targets for pharmacotherapy; and

3. They should have an awareness of the limitations of evidence-based pharmacotherapy approaches to the treatment of behavioral addictions. 


\title{
TS-5
}

\section{Prevention for problematic gaming: Current knowledge and priorities}

\author{
Daniel Luke King*
}

The University of Adelaide Department / Institute Section: Psychology

daniel.king@adelaide.edu.au

Globally, there is growing recognition of the harms generated by problematic gaming and gaming disorder. Research suggests that some individuals, such as adolescents, are more vulnerable than others to developing unhelpful gaming habits, which may progress to addiction in some cases. Public health recommendations have included delaying gaming involvement and restricting screen time during early developmental periods of life. However, the high accessibility and affordability of gaming and other digital technologies across home, work, and school domains has often made such recommendations difficult to apply in practice. In some regions, prevention programs for adolescents have been developed to reduce habitual use of gaming and related activities. This talk will summarise some of the current research, strategies and guidelines on prevention for gaming-related issues, including some critical reflections on what measures appear to be most effective for whom. A useful starting point in this area is clarifying the specific problem to address, which may require acknowledging that gaming differs in many important ways from other activities recognised as potentially addictive. Prevention for young people must take into account important individual-level, peer and family influences, and cultural and environmental influences on gaming behavior. Addressing gaming-related problems effectively requires the sustained effort and collaboration of multiple areas of influence, including families and peers, schools, health providers, government bodies, and the industries that provide online content. 


\section{AUTHOR INDEX}

\begin{tabular}{|c|c|c|c|}
\hline Aboujaoude, E. & $\begin{array}{l}7 \text { (SY2-1); } \\
110(\text { IO3-2) }\end{array}$ & Brand, M. & $\begin{array}{l}19 \text { (SY7-1); } \\
38 \text { (SY15-4); }\end{array}$ \\
\hline Adair, C. & 87 (SY34-4) & & 72 (SY27-5) \\
\hline Ahn, J.-S. & 175 (P18) & Brandtner, A. & 130 (IO9-2) \\
\hline Aikawa, T. & 24 (SY9-1) & Brevers, D. & 77 (SY30-3) \\
\hline Albani, G. & 126 (IO8-2); & Burleigh, T. L. & 44 (SY17-4) \\
\hline & 127 (IO8-3) & Buyukcorak, M. & $90(\mathrm{IO} 11-2)$ \\
\hline Alho, H. & 41 (SY16-4) & Caillon, J. & $120($ IO6-1) \\
\hline Antons, S. & 16 (SY5-5) & Calado, F. & $114(\mathrm{IO} 4-3)$ \\
\hline Apidechkul, T. & 146 (IO14-1) & Carragher, N. & 59 (SY23-2) \\
\hline Arai, K. & 62 (SY24-3) & Challet-Bouju, G. & $113(\mathrm{IO} 4-2)$ \\
\hline Aricak, O. T. & 131 (IO9-4); & Chamberlain, S. & 17 (SY6-1) \\
\hline & $142(\mathrm{IO} 12-5)$ & Chan, E. M. L. & 203 (P58) \\
\hline Asakura, T. & 14 (SY4-4) & Chang, H.-L. & $168(\mathrm{P} 8)$ \\
\hline Asami, Y. & 200 (P54) & Chen, L. & 63 (SY25-1) \\
\hline Assanangkornchai, S. & 93 (SY37-3) & Cheng, L. & 28 (SY11-1) \\
\hline Baba, T. & 47 (SY19-1) & Cheong, A. W. L. & 29 (SY11-3) \\
\hline Baggio, S. & $152(\mathrm{IO} 15-4)$ & Choi, I. Y. & 75 (SY28-4); \\
\hline Baumgartner, C. & 169 (P10) & & 197 (P49) \\
\hline Becker, B. & 56 (SY22-2) & Choi, J.-S. & 73 (SY28-2); \\
\hline Berdibayeva, S. & 198 (P51) & & 180 (P26); \\
\hline Bhang, S.-Y. & $\begin{array}{l}9 \text { (SY3-1); } \\
166(\mathrm{P} 4)\end{array}$ & & $\begin{array}{l}181(\mathrm{P} 27), \\
(\mathrm{P} 28)\end{array}$ \\
\hline Bilke-Hentsch, O. & 105 (IO1-5); & Choi, S. & $171(\mathrm{P} 13)$ \\
\hline & $110(\mathrm{IO} 3-3)$ & Choi, T. Y. & 46 (SY18-3) \\
\hline Billieux, J. & 21 (SY7-5); & Choliz, M. & 121 (IO6-3) \\
\hline & $151(\mathrm{IO} 15-2)$ & Chun, J. & 81 (SY32-2) \\
\hline Blaszczynski, A. & $2($ PL3) & Chung, S. & 52 (SY21-1) \\
\hline Blinka, L. & $133(\mathrm{IO} 10-2)$ & Chung, Y.-J. & 189 (P38) \\
\hline Blycker, G. & 65 (SY25-5) & Clement, T. J. H. & 30 (SY11-4) \\
\hline Boer, M. & 99 (SY39-4) & Corazza, O. & 157 (IO16-4) \\
\hline Boonen, $\mathrm{H}$. & 11 (SY3-4) & Cornil, A. & 115 (IO4-5) \\
\hline Böthe, B. & 15 (SY5-1); & Cui, F. & 199 (P52) \\
\hline & 37 (SY15-1) & Deleuze, J. & $125(\mathrm{IO} 7-5)$ \\
\hline Bottel, L. & $104(\mathrm{IO} 1-3)$ & Demeshkant, N. & 148 (IO14-3) \\
\hline & & Dias, P. & $\begin{array}{l}161 \text { (IO17-3); } \\
201 \text { (P55) }\end{array}$ \\
\hline
\end{tabular}




Dong, G.
Dreier, M.
Dullur, P.
Efrati, Y.
Engel, J.
Farkash, M.
Farnam, R.
Fernandez, D. P.
Firdaus, K. K.
Flayelle, M.
Fraumeni-McBride, J. P.
Frimerman, L.
Furuno, S.
Giacomo, E. D.
Giardina, A.
Gola, M.

Gola, M.

Grall-Bronnec, M.

Grant, J. E.

Grant, J.

Greer, N.

Griffiths, M.

Grubbs, J. B.

Hae-Kook, L.

Han, D. H.

Hao, W.

Harada, T.

$\mathrm{He}, \mathrm{Q}$.

Higuchi, S.

Hing, $N$.

Hodgins, D. C.

Hung, C. C.

Hussain, Z.

Ikeda, F.
101 (SY40-3);

129 (IO9-1)

131 (IO9-3)

85 (SY34-1)

140 (IO12-2)

64 (SY25-2)

193 (P44)

103 (IO1-2)

119 (IO17-1)

188 (P36)

160 (IO17-2)

140 (IO12-1)

200 (P53)

68 (P15)

137 (IO11-3)

135 (IO10-5)

65 (SY25-4);

69 (SY27-1)

67 (SY26-2)

12 (SY6-3)

5 (SY1-2);

208 (TS-4)

122 (IO6-4)

122 (IO6-5);

162 (IO17-4)

6 (SY1-3);

38 (SY15-3);

51 (SY20-3)

175 (P19)

177 (P22)

34 (SY13-3)

26 (SY10-1);

27 (SY10-2)

76 (SY30-1)

58 (SY23-1)

55 (SY21-4);

112 (IO4-1)

3 (PL5);

40 (SY16-2)

89 (SY36-2)

31 (SY12-2)

79 (SY31-4)
Inamura, A.

24 (SY9-2)

Iriki, A.

120 (IO6-2)

Iskandar, S. M.

125 (IO8-1);

134 (IO10-3)

Isobe, M.

18 (SY6-2)

Israelashvili, M. 103 (IO1-1)

Jiang, Q.

143 (IO13-1);

144 (IO13-2)

Joe, K. H.

67 (SY26-3)

Jung, Y.-C.

100 (SY40-1)

Kamimura, E.

36 (SY14-2)

Katagami, M.

80 (SY32-1)

Katayama, M.

107 (IO2-3)

Kato, T. A.

Kawabe, K.

Kern, L.

Kido, M.

47 (SY18-4)

45 (SY18-1)

129 (IO8-5);

165 (P3)

36 (SY14-1);

60 (SY36-4)

Killick, E. A. 205 (P60)

Kim, D.

187 (P35)

Kim, D.-J.

10 (SY3-2);

22 (SY8-2);

73 (SY28-1);

183 (P30)

Kim, J.-H.

101 (SY40-2);

173 (P16)

152 (IO15-3)

Kim, S.-G.

53 (SY21-2)

Kim, Y.

202 (P56)

Kindaichi, Y

34 (SY13-2);

209 (TS-5)

Kinjo, A.

96 (SY38-3)

Kiraly, O.

144 (IO13-3)

Ko, C.-H.

2 (PL2);

16 (SY8-4)

Ko, H.-C.

32 (SY12-4)

Kobara, K.

14 (SY4-5);

49 (SY19-4)

Koizumi, N.

13 (SY4-2)

Koning, I. M.

98 (SY39-1)

Koronczai, B.

136 (IO11-1) 
Kotbagi, G.

Kowalewska, E.

Kraus, S. W.

Kun, B.

Kuriki, S.

Kurniasanti, K. S.

Kuss, D. J.

Kweon, Y.-S.

Laskowski, N. M.

Lau, J. T. F.

Le, H. K.

Lee, D.

Lee, H. K.

Lee, J.

Lee, S.-K.

Lee, $\mathrm{T}$.

Limbrick-Oldfield, E. H.

Liu, T.

Liu, T.-Q.

Lopez-Fernandez, O.

Luquiens, A.

Maia, B. R.

Matsuzaki, T.

Mead, D. K.

Melvyn, Z. W.

Melzer, A.

Mennig, $\mathrm{M}$.

Mihara, S.

Mimura, M.

Montag, C.

Moretta, T.

Morita, N.

Mueller, S. M.
156 (IO16-3)

50 (SY20-2)

6 (SY1-4);

52 (SY20-5)

158 (IO16-5)

70 (SY27-3)

92 (SY37-1)

44 (SY17-5)

139 (IO11-6);

184 (P31)

148 (IO14-4)

153 (IO15-5)

86 (SY34-3)

74 (SY28-3);

109 (IO3-1)

3 (PL4)

186 (P34)

83 (SY33-1)

23 (SY8-3);

194 (P46)

76 (SY30-2)

54 (SY21-3)

185 (P32)

42 (SY17-2);

119 (IO5-6)

66 (SY26-1)

193 (P45);

196 (P48)

40 (SY16-3);

68 (SY26-4)

141 (IO12-3)

29 (SY11-2)

134 (IO10-4)

179 (P25)

83 (SY33-2);

94 (SY37-5)

116 (IO5-1)

33 (SY12-5);

57 (SY22-3)

177 (P21)

79 (SY31-3)

150 (IO15-1)
Müller, A.

20 (SY7-3);

147 (IO14-2)

Müller, K. W.

84 (SY33-3);

207 (TS-3)

Murase, H.

149 (IO14-5)

Mustonen, T.

186 (P33)

Nakayama, H.

11 (SY3-3)

5 (SY1-1)

195 (P47)

Noda, Y.

Nomura, K.

27 (SY10-3)

Nuyens, F. M.

Oh, S.

Ostendorf, S.

Park, A. R.

Park, A.

138 (IO11-4),

(IO11-5)

182 (P29)

55 (SY22-1)

169 (P9)

48 (SY19-2)

Pekal, J.

111 (IO3-4);

145 (IO13-4)

Petricevic, N. $190(\mathrm{P} 40)$

Pontes, H. M.

Potenza, M. N.

Poznyak, V. B.

154 (IO15-6)

7 (SY1-5);

15 (SY5-3);

19 (SY7-2);

39 (SY15-5);

39 (SY16-1);

70 (SY27-2);

207 (TS-2)

Prever, F.

1 (PL1)

128 (IO8-4)

Ratta-apha, W.

90 (SY36-3)

Rehbein, F.

33 (SY13-1);

60 (SY23-4)

Reid, R. C.

51 (SY20-4)

Rogier, G.

114 (IO4-4);

170 (P11);

171 (P12)

Roh, S.

94 (SY37-4);

197 (P50)

Rumpf, H.-J.

20 (SY7-4);

60 (SY23-3)

Russell, A. M. T. 108 (IO2-5)

Ryding, F. C. 


\begin{tabular}{|c|c|c|c|}
\hline \multirow{3}{*}{$\begin{array}{l}\text { Sariyska, R. } \\
\text { Sato, T. }\end{array}$} & 57 (SY22-4) & Trotzke, $\mathrm{P}$. & $154(\mathrm{IO} 16-1)$ \\
\hline & 13 (SY4-3); & Tsuneoka, T. & 78 (SY31-2) \\
\hline & 26 (SY9-4) & Tsurumi, K. & 61 (SY24-1); \\
\hline Saunders, J. B. & 206 (TS-1) & & 92 (SY37-2) \\
\hline Scherer, L. & 43 (SY17-3) & Uhl, A. & $106(\mathrm{IO} 2-1)$ \\
\hline Ševčíková, A. & 133 (IO10-1) & van den Eijnden, R. J.J.M. & 98 (SY39-2) \\
\hline Sharman, S. & 107 (IO2-2) & Varescon, I. & 167 (P6); \\
\hline Sharpe, M. & 142 (IO12-4) & & 168 (P7) \\
\hline Shi, J. & 105 (IO1-4) & Vasiliu, O. & 164 (P1), (P2); \\
\hline Shin, Y. & 95 (SY38-1) & & 166 (P5); \\
\hline Shirasaka, T. & $\begin{array}{l}22 \text { (SY8-1); } \\
88 \text { (SY36-1) }\end{array}$ & & $\begin{array}{l}174 \text { (P17); } \\
192 \text { (P43) }\end{array}$ \\
\hline Sindermann, C. & $30($ SY12-1) & Veit, M. & 64 (SY25-3) \\
\hline So, R. & 49 (SY19-3) & Wang, G. & $124(\mathrm{IO} 7-4)$ \\
\hline Sora, I. & $179(\mathrm{P} 24)$ & Wang, M. P. & $116(\mathrm{IO} 5-2)$ \\
\hline Starcevic, V. & $\begin{array}{l}8 \text { (SY2-3); } \\
86 \text { (SY34-2) }\end{array}$ & & $\begin{array}{l}117 \text { (IO5-3); } \\
162 \text { (IO17-5) }\end{array}$ \\
\hline Stark, R. & 16 (SY5-4) & Watanabe, I. & 62 (SY24-4) \\
\hline Stavropoulos, V. & 42 (SY17-1) & Wegmann, E. & 31 (SY12-3) \\
\hline Sugawarad, N. & 87 (SY35-1) & Wells, T. J. & 191 (P41); \\
\hline Suryani, E. & 202 (P57) & & 192 (P42) \\
\hline Szabo, A. & 155 (IO16-2) & Wu, A. M. S. & 97 (SY38-4); \\
\hline Szász-Janocha, C. & 35 (SY13-4) & & 108 (IO2-4); \\
\hline Takahashi, H. & 4 (PL6) & & 112 (IO3-5); \\
\hline Takahashi, M. & 176 (P20) & & 188 (P37) \\
\hline Takahashi, Y. & 190 (P39) & WU, M. S. M. & 204 (P59) \\
\hline Tam, P. G. & 9 (SY2-4) & Yamada, M. & 118 (IO5-4) \\
\hline Tamura, K. & 87 (SY35-2) & Yamada, R. & 82 (SY32-4) \\
\hline Tanabe, H. & 12 (SY4-1) & Yan, E. & $172(\mathrm{P} 14)$ \\
\hline Tanaka, M. & 25 (SY9-3) & Yang, Z. & 118 (IO5-5) \\
\hline Tanaka, N. & 80 (SY31-5) & Yee, A. & 91 (SY36-5) \\
\hline Tanaka, Y. & 28 (SY10-4) & Yim, H. W. & 81 (SY32-3); \\
\hline Tateno, M. & 45 (SY18-2) & & 96 (SY38-2) \\
\hline Thaikla, K. & $178(\mathrm{P} 23)$ & Yokomitsu, K. & 37 (SY14-3) \\
\hline Throuvala, M. A. & 123 (IO7-2), & Yokotani, K. & 88 (SY35-4) \\
\hline & $(\mathrm{IO} 7-3)$ & Yu, L. & 99 (SY39-3) \\
\hline Tokumasu, T. & 85 (SY33-4) & Zhang, J. & 71 (SY27-4) \\
\hline Toyama, T. & 78 (SY31-1) & Zhang, X. & 132 (IO9-5) \\
\hline
\end{tabular}




\title{
Guidelines for Authors
}

\section{GENERAL INFORMATION}

\section{Aims and scope}

The aim of the Journal of Behavioral Addictions is to create a forum for the scientific information exchange with regard to behavioral addictions. The journal is a broad focused interdisciplinary one that publishes manuscripts on different approaches of non-substance addictions, research reports focusing on the addictive patterns of various behaviors, especially disorders of the impulsive-compulsive spectrum, and also publishes reviews in these topics. Coverage ranges from genetic and neurobiological research through psychological and clinical psychiatric approaches to epidemiological, sociological and anthropological aspects.

\section{Contact}

In case of any query, please, feel free to contact:

jba@ppk.elte.hu

\author{
Address of the Editorial Office \\ Zsolt Demetrovics, Editor-in-Chief \\ Institute of Psychology, Eötvös Loránd University \\ Journal of Behavioral Addictions \\ Izabella utca 46, H-1064 Budapest, Hungary \\ Phone: +36 30 9761097; Fax: +36 14612697
}

\section{Conditions of submission and copyright issues}

Submission of a paper to the Journal of Behavioral Addictions will be taken to imply that it represents original work not previously published, that it is not being considered elsewhere for publication, and that if accepted for publication it will not be published elsewhere in the same form, in any language, without the consent of editor and publisher. JBA is an Open Access journal. When publishing with Open Access the final published version can be archived and made publicly accessible by the author immediately after first publishing of the article. Copyright and publishing rights (other than the right of first publishing) are retained by the author, without any restrictions.

\section{Article processing charges}

The Journal of Behavioral Addictions is an Open Access journal. The corresponding author of every manuscript submitted after July 1, 2018 is requested to pay the Article Processing Charge (APC) of $\mathbf{8 5 0}$ EUR (including VAT) to cover the publishing costs of the paper. Aka- 
démiai Kiadó offers 50\% discount for Editorial Board members, Hungarian institutions, and institutions of low- and middle-income countries. We offer $50 \%$ discount for Commentaries. We also offer $15 \%$ discount for corresponding authors who are members of the International Society for the Study of Behavioral Addictions (ISSBA). Discounts may not be combined. Manuscripts with outstanding payment will not be published until the balance is cleared.

Once your paper is accepted for publication in the Journal of Behavioral Addictions you will be requested to enter our online payment platform (http://apc.akademiai. hu), where after registration You can upload license agreement and transfer the fee. Article processing will commence after the amount due has been remitted to our account. (Please note that there is no peer review charge, the only applicable fee is the article processing charge for authors of accepted papers.)

Open Access policy of the journal includes the free and unrestricted self-archiving of the final published version of papers on the Author's webpage or on the Author's institutional repository with open or restricted access. When self-archiving or communicating a paper via e-mail, the Author should quote the correct citation and enclose a link to the published article (http://dx.doi.org/[DOI of the Article without brackets]).

\section{Submission of manuscripts}

Authors should submit their manuscripts using the online manuscript submission and peer review system Editorial Manager. Details are available online at http://www. editorialmanager.com/jbadd. All manuscript submissions should be accompanied by a Cover letter and a Title page. The Cover letter may include a list of suggested reviewers, their affiliations, and their e-mail addresses. The Title page should include statements regarding Funding sources, Authors' contribution, Conflict of interest, and Ethics. Following the blind review process and the acceptance of the manuscript, these statements will be added to the published paper.

\section{Preferred conditions of manuscripts}

The Journal of Behavior Addictions strives to publish high quality papers. To ensure this goal, we apply some basic principles when reviewing a manuscript. Not fulfilling these principles may lead to desk rejection of a submitted manuscript.

JBA has a strong preference for studies that apply experimental or observational design (i.e., not cross-sectional design) and a random or systematic sampling method (i.e., not convenient sampling). Originality (raising new questions and hypotheses) plays an especially important role in the review process of manuscripts that use convenient sampling and crosssectional design.

In case of psychometric studies, the sole adaptation of already existing measures to another single language is not preferred. However, psychometric studies that lead to new results regarding the construct or incremental validity and/or its further theoretical development are of interest. 


\section{Review of the manuscripts}

Each paper will be read by at least two referees. The Journal of Behavioral Addictions applies a double-blind review process, in which both the authors' and the reviewers' identities are concealed. It is the authors' responsibility to see that the manuscript itself contains no clues to the authors' identity.

\section{Authorship}

All persons designated as authors should qualify for authorship. Each author should have participated sufficiently in the work to take public responsibility for the content. The corresponding author affirms that he or she had access to all data from the study, both what is reported and what is unreported, and also that he or she had complete freedom to direct its analysis and its reporting, without influence from the sponsors. The corresponding author also affirms that there was no editorial direction or censorship from the sponsors. Preparation of drafts of manuscripts by employees of the sponsor who are not listed as authors is expressly prohibited.

\section{Disclosure of financial relationships}

Disclosure of financial relationships is required at the time of submission and provisional acceptance of all manuscripts, including original articles, reviews, commentaries, and book reviews. The authors are responsible for informing the Journal of any additional financial relationships that may arise prior to the date of publication of their paper. Financial support for the study is always disclosed on the Title page, whether from governmental, nonprofit, or commercial sources. Non-financial forms of support, such as drugs, analytic support, or other assistance with preparation of the manuscript, must also be acknowledged. All authors must individually disclose all financial relationships, whether or not directly related to the subject of their paper. Such reporting must include all equity ownership, profit-sharing agreements, royalties, patents, and research or other grants from private industry or closely affiliated nonprofit funds. For income from pharmaceutical companies, the purpose must be specified. Reporting should encompass the previous 36 months. If an author has no interests to disclose, this must be explicitly stated in the Title page.

The Editors will review all author statements of financial support to determine if there is evidence of bias from these sources. If it appears that there may be, then further review and possible rejection of the manuscript may occur. Authors are encouraged to contact the Editor at any stage in the manuscript review process if they believe that they have relationships that require review. 


\section{Ethical considerations}

The Journal of Behavioral Addictions and Akadémiai Kiadó are committed to the protection of animal and human research subjects and ethical practices in science publishing. Studies submitted to the Journal of Behavioral Addictions must have been conducted in accordance with the Declaration of Helsinki and according to requirements of all applicable local and international standards. The Journal of Behavioral Addictions declares its support for and follows the ethical principles enshrined in the Farmington Consensus (http://www.parint.org/ isajewebsite/farmington. htm). All authors must conform to the highest standards of ethical conduct in the submission of accurate data, acknowledging the work of others, and divulging potential conflicts of interests. Ethical statements should be included in the Methods section of the manuscript (see later).

\section{PREPARATION OF THE MANUSCRIPTS}

\section{Types of manuscripts}

(1) Full-length reports reporting original results of research within the field of behavioral addictions. A Full-length report typically should not exceed 4000 words for the main body of the paper (i.e., excluding abstract, references, acknowledgements, tables and figures). Manuscripts should be as concise as possible. In special cases manuscripts might exceed the 4000 words limit, however, in these cases a detailed justification is required in the cover letter.

(2) Review articles of specialized topics within the scope of the journal. Typically, these are systematic reviews or critical analyses of a field of research. A Review article typically should not exceed 6000 words (i.e., excluding abstract, references, acknowledgements, tables and figures). Review articles must also have an abstract which follows the same (or similar) structure as the original (full-length) research reports.

(3) Brief reports reporting on research that has progressed to the stage where a preliminary publication is appropriate. Manuscript length for Brief reports is 2000 words (excluding abstract, references, acknowledgements, tables and figures). There should be not more than 2 illustrations (figures or tables) and 30 references.

(4) Commentaries express points of view on scientific matters or published papers. Typically, Commentaries are solicited by the editors, but authors who wish to submit Commentaries are advised to contact the Editor-in-Chief to discuss the suitability of the proposed paper. A Commentary typically should not exceed 2000 words (excluding abstract and references). Commentaries must always have an abstract, which may be unstructured and consists of no more than 100 words.

(5) Case reports. Only exceptional cases will be considered for publication as a single case report. Case reports should describe novel, well-documented findings that will be of help and interest to the practitioner. Case reports typically should not exceed 2000 words (excluding abstract, references, acknowledgements, tables and figures). Case reports always have an abstract, applying the following structure: Background, Case presentation/study, Discussion, Conclusions. The main text should follow this structure as well. 
(6) Book reviews. Books for review should be sent to the Editorial Office of the Journal of Behavioral Addictions. Books are received with the understanding that reviewers selected by the Editor-in-Chief write their independent critical appraisals of the book's content and presentation.

(7) Letters to the Editor. Brief letters (maximum of 1000 words, including references) will be considered for publication. Letters should not include figures and tables.

(8) Editorials are solicited by the editors. An Editorial typically should not exceed 2000 words (excluding abstract, references, acknowledgements, tables and figures).

(9) Our journal does not publish questionnaires.

\section{Manuscript submission requirements}

Manuscripts should be written in English and should be typed in double spacing with wide margins. All submissions have to include a separate Cover letter, a Title page and the manuscript itself.

The Title page should contain the title of the paper, a short running title, the name, the affiliation and the e-mail address of each author, and an indication of which author will be responsible for correspondence. All contact details (address with postal codes and countries, phone, FAX and e-mail) of the corresponding author are required. Abbreviations in the title should be avoided. The Title page should contain the date when the manuscript is submitted.

The Title page must contain the disclosures described below. Following the blind review process and the acceptance of the manuscript these statements will be added to the published paper:

- Funding sources: State even if no funding was obtained by writing "Nothing declared" or "No financial support was received for this study" or any other similar statement.

- Authors' contribution: Please specify each author's contribution to the manuscript. Use the monograms of the authors and specify their roles in preparing the manuscript. You may use the following roles: study concept and design, analysis and interpretation of data, statistical analysis, obtained funding, study supervision, or any other specific role. Also state whether all authors had full access to all data in the study and take responsibility for the integrity of the data and the accuracy of the data analysis.

- Conflict of interest: All authors are requested to disclose any actual or potential conflict of interest including any financial, personal or other relationships with other people or organizations within three years of beginning the work submitted that could inappropriately influence, or be perceived to influence, their work. Also state if no conflict of interest was present by writing "The authors declare no conflict of interest" or "The authors report no financial or other relationship relevant to the subject of this article" or any other similar statement.

- You may add an optional Acknowledgements section for any further information you wish to disclose. 


\section{The format of the manuscript}

Abstracts should not exceed 250 words and should be presented on a separate sheet. With
the exception of Book reviews, Letters to the Editor, Commentaries, and Editorials all manu-
script types must have a structured abstract. Abstracts should be structured with specific sec-
tions describing the Background and aims, Methods, Results, Discussion and Conclusions.

Keywords: Abstracts should be accompanied by three to six key words or phrases that characterize the contents of the paper. These will be used for indexing and data retrieval purposes.

The body of research reports will generally include the following headings: Introduction, Methods, Results, Discussion and Conclusions. Further subheadings are acceptable.

Review papers and Case reports should also use the same (or similar) section headings and subheadings both in the manuscript and in the abstract.

The Methods section should have separate subsections and subheadings for the following methodological information: Participants, Measures, Procedure, Statistical analysis, and Ethics.

All empirical studies must have a short description on the ethics regarding the study. This should be placed at the very end of the Methods section (just before Results) under a separate subheading of Ethics. The Ethics section should contain information on the ethical approval of the study by the Institutional Review Board and the informed consent given by the participants of the study. An example:

\section{Ethics}

The study procedures were carried out in accordance with the Declaration of Helsinki.

The Institutional Review Board of the [Name of the Institute] approved the study. All subjects were informed about the study and all provided informed consent. (If applicable:

Parental consent was sought for those younger than 18 years of age.)

In order to secure anonymity in the manuscript you may mask the name of the Institute, however this must be mentioned in the Title page.

Sections should not be numbered. Please, avoid footnotes.

Text headings: All headings in the text should be set over to the left-hand margin, and the text should begin on the next line. Type first level (sectional) HEADINGS ALL IN CAPITALS. For second and third level headings, only the first letter of the first word should be a capital. Use bold letters for second level headings and italic for third level headings.

References: Style, statistical reporting, and reference citations should conform to the American Psychological Association's guidelines, from the Publication Manual of the American Psychological Association (Sixth Edition). To conform with the APA Publication Manual, sixth edition, references should be alphabetized at the end of the manuscript text. Please make sure that the DOI is provided after each article in the References.

Figures should be numbered with consecutive Arabic numerals, have descriptive captions and be mentioned in the text. Figures should be kept at the end of the manuscript 
text (after references) and an approximate position for each should be indicated in the margin. It is the author's responsibility to obtain permission for any reproduction from other sources. Photographs should be submitted electronically in TIF or JPG format in separate files. Color figures submitted by the authors will appear in color on the web; however, the extra cost of color reproduction in the printed version must be borne by the authors. Authors will receive information regarding the costs from the editor after acceptance of the article.

Tables should be clearly typed with double spacing. Number tables with consecutive Arabic numerals and give each a clear descriptive heading. Avoid the use of vertical rules in tables. Table notes should be typed below the table, designated by superior lower-case letters. Tables should be kept at the end of the manuscript text (after figures) and an approximate position for each table should be indicated in the text.

Proofs: Authors will receive proofs (including figures) by e-mail for correction, which must be returned within 72 hours of receipt.

Page charges: There are no page charges. 